\title{
Model Review and Evaluation for Application in DOE Safety Basis Documentation of Chemical Accidents - Modeling Guidance for Atmospheric Dispersion and Consequence Assessment
}

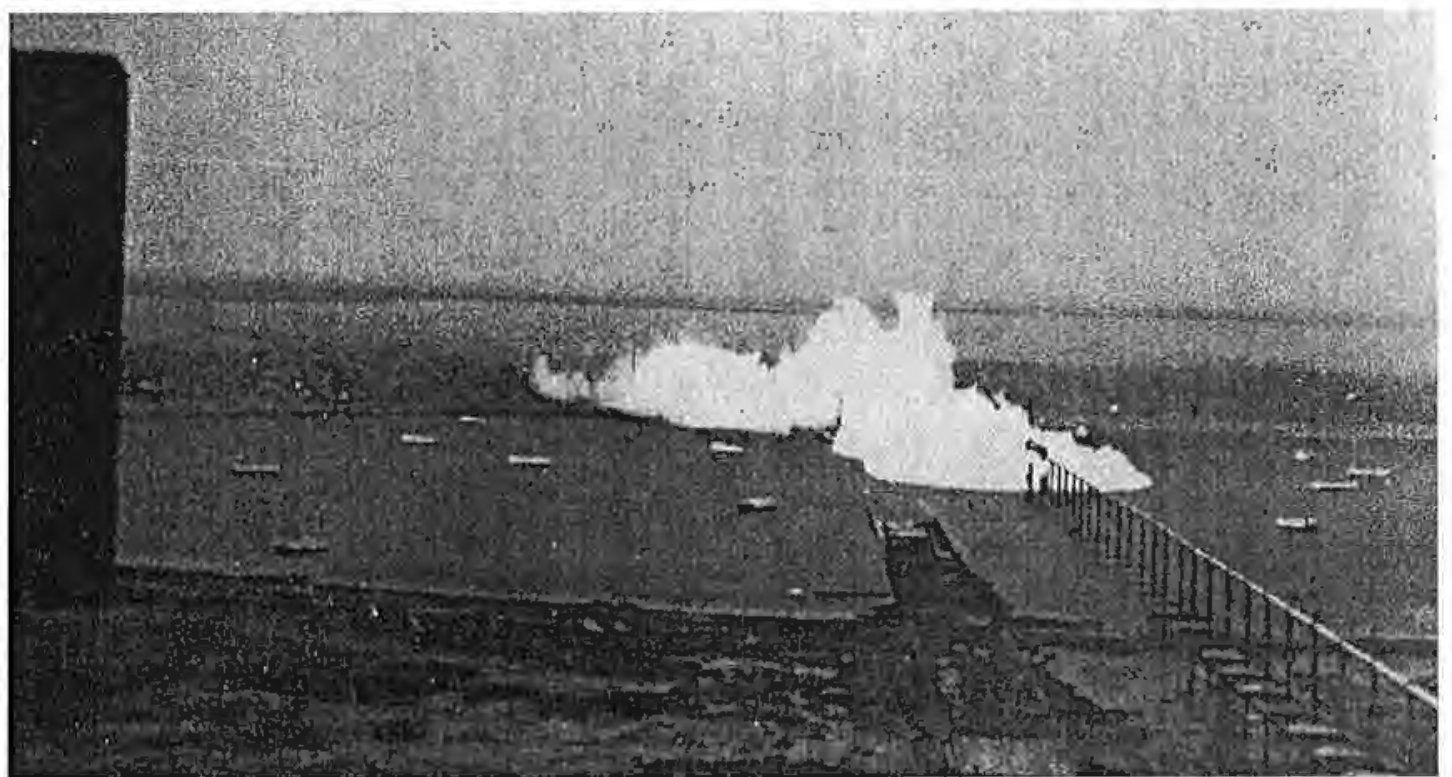

Accident Phenomenology and Consequence Assessment (APAC) Methodology Evaluation Working Group 6 Report

M.A. Lazaro, Co-Chair, Argonne National Laboratory

K. Woodard, Co-Chair, PLG, Inc.

S.R. Hanna, Earth Tech, Inc.

D.J. Hesse, Battelle Columbus Laboratories
J.-C. Huang, Westinghouse Savannah River Co.

J. Lewis, PLG, Inc.

C.A. Mazzola, Stone \& Webster Engineering Group

September 1997

Environmental Assessment Division

Argonne National Laboratory, 9700 South Cass Avenue, Argonne, Illinois 60439

Work sponsored by United States Department of Energy,

Office of Defense Programs, DP-45

under Contract W-31-109-Eng-38 


\section{Argonne National Laboratory}

Argonne National Laboratory, with facilities in the states of Illinois and Idaho, is owned by the United States Government, and operated by the University of Chicago under the provisions of a contract with the Department of Energy. This technical memo is a product of Argonne's Environmental Assessment Division (EAD). For information on the division's scientific and engineering activities, contact:

Diractor, Environmental Assessment Division

Argonne National Laboratory

Argonne, Illinols 60439-4815

Telephone (630) 252-3107

Presented in this technical memo are preliminary results of ongoing work or work that is more limited in scope end depth than that described in formal reports issued by the EAD.

Publishing support services ware provided by Argonne's Information and Publishing Division (for more information, see IPD's home page: http:/www ipd anl.gov/).

\section{Disclaimer}

This report was prepared as an account of work sponsored by an agency of the United States Government. Neither the United States Governıent nor any agency thereof, nor any of their employees, makes any warranty, express or implied, or assumes any legal liability or responsibility for the accuracy, completeness, or usefulness of any information, apparatus, product, or process disciosed, or represents that its use would not infringe privately owned rights. Reference herein to eny specific commercial product, process, or service by trade name, tradernark, menufacturer, or otherwisa, does not necessarily constitute or imply its endorsement, recommendation, or favoring by tha United States Government or any agency thereof. The views and opinions of authors axpressed herein do not necessarily state or reflect those of the United States Government or any agency thereof.

Title Page: Photographic data, Spill No, 23, Plate 5, from TNER.84.041, SPILL TESTS OF LNG AND REFRIGERATED PROPANE ON THE SEA, MAPLIN SANDS, 1980: DISPEASION DATA IGEST; TAIAL 23

Photograph reproduced with the permission of Shell Research Limited.
Reproduced directly from the best available copy.

Available to DOE and DOE Contractors from the Office of Scientific and Technical information, P.O. Box 62, Oak Aidge, TN 37831 ; prices evailable from (423) $576-8401$.

Avallable to the public from the National Technical Information Service, U.S. Department of Commerce, 5285 Port Royal Road, Springfield, VA 22161. 


\section{CONTENTS}

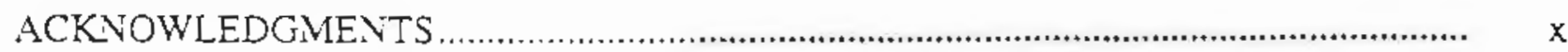

ACRONYMS AND ABBREVIATIONS .................................................................... xi

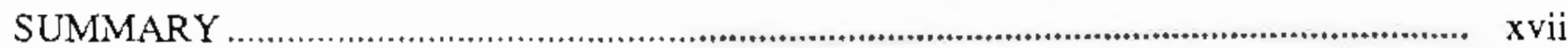

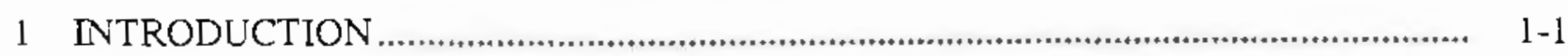

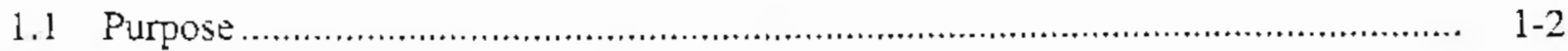

1.2 Objective and Scope .................................................................................. $1-4$

1.2.1 Review Regulatory Requirements........................................................ 1-5

1.2.2 Identify and Select Computer Codes Applicable to APAC Chemical Dispersion and Consequence Assessment................................................ I-5

1.2.3 Develop Comprehensive Basis for Model Reviews................................... 1-5

1.2.4 Develop Sample Problems for Tier I Reviews ............................................ 1-5

1.2.5 Evaluate Computer Models and Compare Test Case Results..................... $1-6$

1.2.6 Identify Best Practices ....................................................................... $1-6$

1.2.7 Develop Conclusions and Recommendations ......................................... 1-7

1.2.8 Identify Needs for Improving Consequence Assessment for SBD .............. 1-7

1.3 DOE Guiding Orders and Other Federal Regulatory Documents .......................... 1-7

1.4 Current Chemical Safety Analysis Practices within the DOE Complex …............... 1-8

2 SELECTED SOURCE RELEASE AND DISPERSION MODELING PRINCIPLES RELEVANT TO CONSEQUENCE ASSESSMENT …......................... 2-1

2.1 Influence of Release Physics and Thermodynamics, and

Substance-Mixture Physical Property Effects ............................................................ 2-1

2.1.1 Subcooled Leaks/Spills and Liquid Jets .................................................... 2-2

2.1.2 Superheated Liquid Jets/Spills and Two-Phase Releases.............................. 2- 2-4

2.1.3 Particulate and Vapor Dry Deposition and Resuspension............................ 2. 2-6

2.1.4 Momentum- or Buoyancy-Dominated Plumes............................................... 2- 2-6

2.1.5 Dense Gas, Neutrally Buoyant, and Positively Buoyant Releases ................ 2- 2-7

2.1.6 Building Wake and Stack/Building Downwash............................................. 2-9

2.2 Meteorological Influences .................................................................................... 2-10

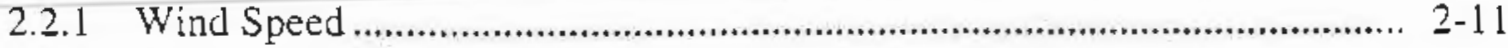

2.2.2 Ambient Temperature, Relative Humidity, and

Atmospheric Pressure........................................................................... 2-12

2.2.3 Atmospheric Turbulence and Diffusion ..................................................... 2-12

2.2.4 Ground-Surface Effects ............................................................................ 2-13 


\section{CONTENTS (Cont.)}

2.3 Role of Hand Calculations .................................................................................. 2-16

2.3.1 Source Geometry Approximations ........................................................... 2-16

2.3.2 Jet Dispersion............................................................................... 2-17

2.3.3 Dense Gas Jet/Plume Dispersion.............................................................. 2-17

2.3.4 Positively Buoyant Plume Rise and Dispersion ........................................... 2-18

2.3.5 Stack and Building Downwash ................................................................. 2-18

2.3.6 Dense Gas Ground-Level Dispersion...................................................... 2-18

2.3.7 Neutrally Buoyant Dispersion ................................................................... 2-19

2.4 Considerations for Release Duration and Averaging, Sampling,

Exposure, and Travel Times......................................................................... 2-19

2.4.1 Release Duration ................................................................................... 2-19

2.4.2 Averaging and Sampling Time.................................................................. 2-20

2.4.3 Exposure Time - Dose/Toxic Load or
Concentration Effects.............................................................................. 2-20

2.4.4 Advection Travel Time ....................................................................... 2-21

2.4.5 Instantaneous Versus Continuous Release ................................................ 2-21

2.4.6 Variability in Approaches ..................................................................... 2-22

3 MODEL SELECTIO:

3.1 Chemical Accident Assessment Problems Faced by Safety Analysts ....................... 3-1

3.2 Code Selection Process ............................................................................................. 3-2

3.3 Surnmary of Codes Selected................................................................................. $3-4$

3.3.1 Tier I Codes ...................................................................................... $3-4$

3.3.2 Tier II Codes ...................................................................................... 3-15

4 BASIS FOR MODEL REVIEW AND EVALUATION …..............................................

4.1 Summary of Requirements and Model Review Basis ............................................. 4-1

4.2 Development of Model Review Worksheets .......................................................... 4-1

4.2.1 General Model Review ................................................................................. 4-2

4.2.2 Specific Model Review ................................................................................ 4-2

4.3 Description of Test Problems ..................................................................................... 4-5

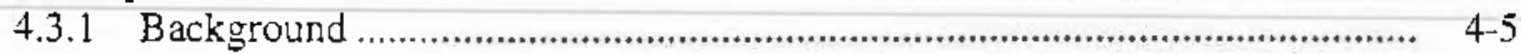

4.3.2 General Rationale Behind Choice of Test Cases.......................................... 4-6

4.3.3 Description of Seven Test Cases ................................................................. 4-7

5 MODEL REVIEW AND COMPARISON RESULTS …............................................. 5 -

5.1 Review Basis Results — Analysis Class .............................................................. 5-1 


\section{CONTENTS (Cont.)}

5.2 Test Problem Findings ………................................................................................ $5-7$

5.2.1 Analysis of Results of Test Cases .............................................................. 5-7

5.2.2 Discussions of Model Comparisons for Each Test Case............................... 5-8

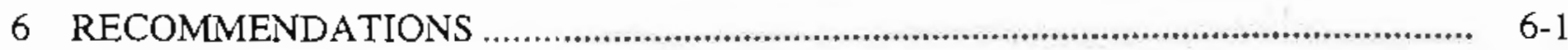

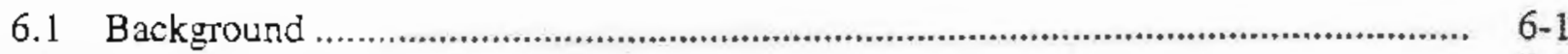

6.2 Good Modeling and Safety Analysis Practices .......................................................... 6-1

6.2.1 General Guidance ............................................................................... 6-2

6.2.2 Specific Guidance ….............................................................................. 6-3

6.3 Chemical Consequence Assessment Model Recornmendations ............................... 6-12

6.3.1 Tier I Models ............................................................................................ 6-14

6.3.2 Tier II Model Recommendations................................................................. 6-29

6.4 Chemical Consequence Assessment Modeling Needs ............................................... 6-30

6.4.1 Expand Tier I Model Reviews...................................................................... 6-30

6.4.2 Conduct a Systematic Model Performance Evaluation with

Field and Laboratory Data...................................................................... 6-31

6.4.3 Address Model Development Needs ......................................................... 6-32

6.4.4 Establish a Safety Analysis Clearinghouse Web Page ……............................ 6-35

6.4.5 Establish Chemical Dispersion Modeling Workshop …................................ 6-36

6.4.6 Consider Establishing a Joint Spills and CDCA Working Group................. 6-37

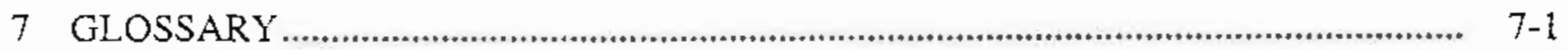

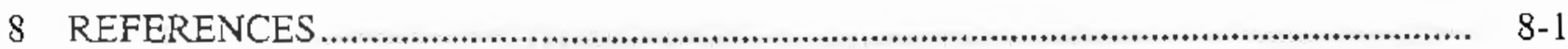

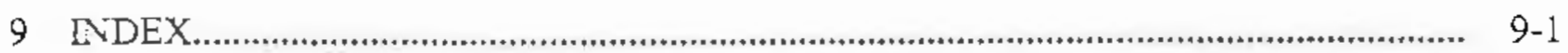

\section{APPENDIXES *}

APPENDIX A: Department of Energy Guiding Orders and Other Regulatory Requirements.

APPENDIX B: Suryey of DOE Sites.......................................................................... B-1

APPENDIX C: Partial List and Inventory of Chemical Use Within

DOE Complex

* Because of size constraints, appendixes prepared in support of this report have not been printed with the document; however, copies of individual appendixes cited in the text may be obtained upon request from Michael Lazaro, Environmental Assessment Division, Argonne National Laboratory, 9700 S. Cass Avenue, Argonne, lllinois. 


\section{CONTENTS (Cont.)}

APPENDIX D: Health Criteria for Use in Computing Hazard Zones

D-1

APPENDLX E: Initial List of Codes Considered for Review by the CDCA Working Group

APPENDIX F: Tier I and II Model Reviews and Evaluations $\mathrm{F}-1$

APPENDIX G: Test Problem Results G-1

APPENDIX H: Bios of Working Group Members $\mathrm{H}-1$

\section{TABLES}

S.1 Public Domain Models Reviewed by the CDCA Working Group ................................... xix

S.2 CDCA Conditional Model Recommendations ........................................................... xxi

1.1 CDCA Working Group Members ............................................................................ 1-4

3.1 Summary of Tier I Codes Selected for Review by the CDCA Working Group

3.2 Summary of Tier II Codes Selected for Review by the CDCA Working Group.

4.1 Examples of Analysis Classes for Five Analysis Levels

4.2 Input/Output Specification for Accident Scenario Cases

5.1 Programmatic Applications for Tier I Models ................................................................ 5-1

5.2 Comparison of Tier I Model Attributes ...................................................................... 5-2

5.3 Test Cases for Tier I Model Accident Scenario Applications ….................................. 5-8

5.4 Comparison of Results for Conditionally Recommended Codes

6.1 Suggested Typicaily Required Default Modeling Parameter Values for DBI Assessments 


\section{TABLES (Cont.)}

6.2 General Observations of the Most Likely Worst-Case Meteorology

Influencing Dispersion and Reiease Rate for Various Release Types

6.3 Representative Surface Roughness and Friction Velocity Values

in Constant Shear Stress Layer

6.4 Summary of Tier I Model Recommendations $6-13$

6.5 Tier II Models Conditionally Recommended for Tier I Review Consideration 6-29

\section{FIGURES}

1.1 Model Review/Evaluation Framework for the CDCA Working Group ..................... 1-6

2.1 Phenomenological Progression for Short- and Long-Duration Releases .................... 2-3

5.1a Base Case: Predicted Ground-Level Centerline Concentration vs.

Downwind Distance for Transient Pool Evaporation - 10 Minute Average

5.1b Base Case: Predicted Ground-Level Centerline Concentration vs.

Downwind Distance for Transient Pool Evaporation -

Instantaneous Concentration

5.1c Base Case: Predicted Ground-Level Centerline Concentration vs.

Downwind Distance for Transient Pool Evaporation - 5 Minute Average

5.1d Base Case: Predicted Ground-Level Centerline Concentration vs.

Downwind Distance for Transient Pool Evaporation - 15 Minute Average

5.1e Base Case: Predicted Ground-Level Centerline Concentration vs.

Downwind Distance for Transient Pool Evaporation - 30 Minute Average

5.1f Base Case: Predicted Ground-Level Centerline Concentration vs.

Downwind Distance for Transient Pool Evaporation - 60 Minute Average

5.2 Variant 1: Predicted Ground-Level Centerline Concentration vs.

Downwind Distance for Transient Pool Evaporation - Neutral Stability/

Stronger Wind Speed 


\section{FIGURES (Cont.)}

5.3 Variant 2: Predicted Ground-Level Centerline Concentration vs.

Downwind Distance for Transient Pool Evaporation - Greater

Surface Roughness.

5.4a Base Case: Predicted Ground-Level Centerline Concentration vs.

Downwind Distance from Steady Evaporating Pool

5.4b Base Case: Lateral Distance from Centerline to $30 \mathrm{ppm}$ Prediction

5.5a Variant 1: Predicted Ground-Level Centerline Concentration vs.

Downwind Distance from Steady Evaporating Pool.....

5.5b Variant 1: Lateral Distance from Centerline to $30 \mathrm{ppm}$ Prediction.

5.6a Variant 2: Predicted Ground-Level Centerline Concentration vs.

Downwind Distance from Steady Evaporating Pool.

5.6b Variant 2: Lateral Distance from Centerline to $30 \mathrm{ppm}$ Prediction.

5.7 Base Case: Predicted Ground-Level Downwind Centerline Concentration vs. Downwind Distance from Slowly Evaporating Contained Liquid Spill

5.8 Variant 1: Predicted Ground-Level Downwind Centerline Concentration vs. Downwind Distance from Slowly Evaporating Contained Liquid Spill

5.9 Variant 2: Predicted Ground-Level Downwind Centerline Concentration vs. Downwind Distance from Slowly Evaporating Contained Liquid Spill

5.10 Base Case: Predicted Centerline Concentration vs. Downwind Distance from Vertical Momentum Jet.

5.11 Variant 1: Predicted Centerline Concentration vs. Downwind Distance from Vertical Momentum Jet.

5.12 Variant 2: Predicted Centerline Concentration vs. Downwind Distance from Vertical Momentum Jet.

5.13 Base Case: Predicted Ground-Level Downwind Centerline Concentration vs. Downwind Distance from Buoyant Release

5.14 Variant I: Predicted Ground-Level Downwind Centerline Concentration vs. Downwind Distance from Buoyant Release 


\section{FIGURES (Cont.)}

5.15 Variant 2: Predicted Ground-Level Downwind Centerline Concentration vs. Downwind Distance from Buoyant Release

5.16 Base Case: Predicted Ground-Level Downwind Centerline Concentration vs. Downwind Distance from Horizontal Jet

5.17 Variant 1: Predicted Ground-Level Downwind Centerline Concentration vs. Downwind Distance from Horizontal Jet

5.18 Variant 2: Predicted Ground-Level Downwind Centerline Concentration vs. Downwind Distance from Horizontal Jet

5.19a Base Case: Predicted Ground-Level Downwind Centerline Concentration vs. Downwind Distance from Heavy Aerosol Release

$5.19 b$ Base Case: Predicted Ground-Level Crosswind $100 \mathrm{~m}$ Centerline Concentration from Heavy Aerosol Release.

5.19c Base Case: Predicted Ground-Level Crosswind $200 \mathrm{~m}$ Centerine Concentration from Heavy Aerosol Release.

5.19d Base Case: Predicted Ground-Level Crosswind $300 \mathrm{~m}$ Centerline Concentration from Heavy Aerosol Release

5.20 Variant 1: Predicted Ground-Level Downwind Centerline Concentration vs. Downwind Distance from Heavy Aerosol Release

5.21 Variant 2: Predicted Ground-Level Downwind Centerline Concentration vs. Downwind Distance from Heavy Aerosol Release

6.1 Example of "Decision-Tree" Approach to Selecting Appropriate Dispersion Models.

6.2 Possible Prototype APAC Web Page 


\section{ACKNOWLEDGMENTS}

Members of the Chemical Dispersion and Consequence Assessment (CDCA) Working Group of the Accident Phenomenology and Consequence Assessment (APAC) Methodology Evaluation Program express their gratitude to all members of the APAC Executive Committee for the valuable advice, support, and general guidance conveyed to the working group chairs and for their peer review contributions to this report. The members of this distinguished group are:

$\begin{array}{ll}\text { Dae Y. Chung, Chair } & \text { U.S. Department of Energy (DOE) (DP-45) } \\ \text { Farid Bamdad } & \text { Defense Nuclear Facilities Safety Board (DNFSB) } \\ \text { Sandra J. Brereton } & \text { Lawrence Livermore National Laboratory (LLNL) } \\ \text { Michael A. Lazaro } & \text { Argonne National Laboratory (ANL) } \\ \text { Georges A. Melhem } & \text { Arthur D. Little } \\ \text { Kevin R. O'Kula } & \text { Westinghouse Savannah River Co. } \\ \text { Ingle K. Paik } & \text { Westinghouse Savannah River Co. } \\ \text { Louis Restrepo } & \text { Omicron, Safety and Risk Technology } \\ \text { Jay Spore } & \text { Los Alamos National Laboratory (LANL) } \\ \text { Thomas Tuccinardi } & \text { DOE (NN-60) } \\ \text { Keith Woodard } & \text { PLG, Inc. }\end{array}$

We would like to acknowledge the contributions from Hua Wang at ANL and Joe Chang at Earth Tech for their assistance in setting up and running some of the Tier I codes for the seven test case accident scenarios. We would also like to thank several of the model developers for their views and feedback on the reviews of their codes.

The CDCA Working Group would like to express special appreciation to those who volunteered to provide peer review or help find peer reviewers for this report:

Sanford Bloom

D. Nick Daish

Albert Dietz

Nijs Jan Duijm

Robin Hall

Prof. Jerry Havens

Francis Rowsome

Prof. Michael Schatzmann

Joe Touma
Martin Marietta Energy Systems

Cambridge Environmental Research

Consultants, Scientific Model Evaluation of

Dense Gas Dispersion Models Group (SMEDIS)

U.S. Department of Energy (NN-60)

Riso

WS Atkins, Safety and Reliability,

SMEDIS Group

University of Arkansas

U.S. Department of Energy (DP-45)

University of Hamburg, Meteorological Institute,

SMEDIS Group

U.S. Environmental Protection Agency 


\section{ACRONYMS AND ABBREVIATIONS}

$\mathrm{ABP}$

ACSA

ADAM

$\mathrm{ADI}$

ADPIC

ADREA-HF

AEA

AERCLOUD.

AEROPLUME/HFPLUME

AES

AFB

AFTOX

AIChE

AIHA

ALOHA

$\mathrm{ANL}$

ANSI

APAC

API

AQPAC

$A R A C$

ARAP

ARB-OME

ARCHIE

$\mathrm{ARF}$

ARL

ARR

ASA

ASCII

ASCOT

BDBI

$\mathrm{BIO}$

BLAST

BLEVE atmospheric boiling point

accident consequence/safety analysis

Air Force Dispersion Assessment Model (computer code)

alternating direction implicit

Advection Particle In-Cell (computer code)

a three-dimensional volume dense-gas model for complex terrain

Atomic Energy Associates Consultancy Services

Aerosol Cloud Model (computer code)

pressurized jet computer modules used with HGSYSTEM

Atmospheric Environment Service

Air Force Base

Air Force Toxic chemical dispersion model (computer code)

American Instítute of Chemical Engineers

American Industrial Hygiene Association

Areal Locations of Hazardous Atmospheres (computer code)

Argonne National Laboratory

American National Standards Institute

Accident Phenomenology and Consequence

American Petroleum Institute

Air Quality Prediction Atmospheric Code (computer code)

Atmospheric Release Advisory Capability (computer code)

Aeronautical Research Associates of Princeton

Air Resource Board-Ontario Ministry of the Environment

Automated Resource for Chemical Hazard Incident Evaluation (computer code)

airborne release fraction

Air Resources Laboratory

airborne release rate

Auditable Safety Analysis

American Standard Code for Information Interchange

Atmospheric Srudies over Complex Terrain

beyond design basis incident

Basis for Interim Operations

explosion damage assessment module used with MARSS

boiling liquid, expanding vapor explosion 


\begin{tabular}{|c|c|}
\hline CALMET & CALPUFF's meteorological preprocessor \\
\hline CALPUFF & California Puff (computer code) \\
\hline CAPTEX & Cross-Appalachian Tracer Experiment \\
\hline CASRAM & $\begin{array}{l}\text { Chemical Accident Stochastic Risk Assessment Model (computer } \\
\text { code) }\end{array}$ \\
\hline CASRAM-SC & $\begin{array}{l}\text { Chemical Accident Stochastic Risk Assessment Model-Single Case } \\
\text { (computer code) }\end{array}$ \\
\hline CCPS & Center for Chemical Process Safety \\
\hline $\mathrm{CDCA}$ & $\begin{array}{l}\text { Chemical Dispersion and Consequence Assessment (Working } \\
\text { Group) }\end{array}$ \\
\hline CERC & Cambridge Environmental Research Consultants \\
\hline CG & U.S. Coast Guard \\
\hline CG-MATHEW/ADPIC & $\begin{array}{l}\text { Conjugate-Gradient Mass-Adjusted Three-Dimensional Wind Field/ } \\
\text { Atmospheric Diffusion Particle-in-Cell (computer code) }\end{array}$ \\
\hline CLOUD & Concentration Levels of Unconfined Dispersion (computer code) \\
\hline CPP & Cermak, Peterka, and Petersen, Inc. \\
\hline CPU & central processing unit \\
\hline CRDEC & Chemical Research, Development and Engineering Center \\
\hline $\operatorname{CSU}$ & Colorado State University \\
\hline CTDM & Complex Terrain Dispersion Model \\
\hline CTDMPLUS & CTDM computer code plus algorithm for unstable situations \\
\hline $\mathrm{D} 2 \mathrm{PC}$ & Detonation-Deflagration Plume Concentration Model \\
\hline DATAPROP & Data Properties for two-phase modules used with HGSYSTEM \\
\hline DBI & design basis incident \\
\hline DEGADIS & Dense Gas Dispersion (computer code) \\
\hline DIPPR & Design Institute for Physical Property Reporting \\
\hline DNFSB & Defense Nuclear Facilities Safety Board \\
\hline DOD & U.S. Department of Defense \\
\hline DOE & U.S. Department of Energy \\
\hline DOT & U.S. Department of Transportation \\
\hline DP & Office of Defense Programs (DOE) \\
\hline DRIFT & Dense Releases Involving Flammables or Toxics (computer code) \\
\hline DSWA & Defense Special Weapons Agency \\
\hline EA & environmental assessment \\
\hline EAL & emergency action level \\
\hline EC & Environment Canada \\
\hline EEE & explosions and energetic events \\
\hline $\mathrm{EH}$ & Oflice of Environment, Safety, and Health (DOE) \\
\hline
\end{tabular}




$\begin{array}{ll}\text { EIS } & \text { environmental impact statement } \\ \text { EM } & \text { Office of Environmental Managernent (DOE) } \\ \text { EMGRESP } & \text { Emergency Response (computer code) } \\ \text { EPA } & \text { LiS. Environmental Protection Agency } \\ \text { EPICODE } & \text { Emergency Prediction Information Code (computer code) } \\ \text { EPM } & \text { Emissions Production Model } \\ \text { ER } & \text { Office of Environmental Research (DOE) } \\ \text { ERP } & \text { emergency response planning } \\ \text { ERPG } & \text { Emergency Response Planning Guideline } \\ \text { ESC } & \text { Environmental Software Consultants, Ltd. } \\ \text { ES\&H } & \text { environment, safety, and health } \\ \text { FEM3C } & \text { Finite Element Model, Three-Dimensional, version C } \\ \text { FIREPLUME } & \text { a computer code for modeling plume dispersion from fires } \\ \text { FLADIS } & \text { European dense-gas experiments } \\ \text { FMC } & \text { Ford Motor Company } \\ \text { f-N } & \text { frequency-normality } \\ \text { FORTRAN } & \text { Formula Translator (computer language) }\end{array}$

GAUS 1

GRI

GUI

HACS-R/TRACE

HARM

HASCAL

HEGABOX

HEGADAS

HEGADAS-S/T

HF

HFCT

HGSYSTEM

HMRAD

HOTMAC

HOTSPOT
Gaussian - Version 1

Gas Research Institute

graphical user interface

Hazardous Chemical Soluble-Releases/Toxic Release and Atmospheric Dispersion during Chemical Emergencies (computer codes)

Hazardous Atmospheric Release Model (computer code)

Hazard Assessment System for Consequence Aralysis (SCIPUFF front end)

Heavy Gas Box module used with HGSYSTEM

Heavy Gas Dispersion Model (computer code)

the Steady and Transient components of HEGADAS used with HGSYSTEM

\section{hydroger fiuoride}

Hydrogen Fluoride Continuous and Transient modules used with HGSYSTEM

Heavy Gas System (computer code)

Hazardous Materials Response and Assessment Division

Higher Order Turbulence Model for Atmospheric Circulation (computer code)

Hot (radioactive) Simulations Portable model (computer code) 


NPUFF
ISC
IWAQM
ICO
LANL
LLNL
LNG
LOMPUFF
LPG
LPOOL

MARPLOT

MARSS

MDA

MREF

NASA

NN

NOAA

NRC

NSWC

NWS

OB/DG

ORNL

OSHA

PC

PDF

PERF

$P-G$

PGPLUME

P-G/T

PHAST

PLG
Integrated Puff (computer code)

Industrial Source Complex (computer code)

Interagency Workgroup on Air Quality Modeling

Justification for Continued Operation

Los Alamos National Laboratory

Lawrence Livermore National Laboratory

liquefied natural gas

dispersion model used with MARSS

liquefied propane gas

Liquid Pool multicomponent evaporation module used with HGSYSTEM

Mapping Applications for Response Planning and Local Operational Tasks (computer code)

Meteorological and Range Safety Support (computer code)

Modelers' Data Archive

model review and evaluation form

National Aeronautics and Space Administration

Office of Nonproliferation and National Security (DOE)

National Ocearic and Atmospheric Administration

U.S. Nuclear Regulatory Commission

Naval Surface Warfare Center

National Weather Service

Ocean Breeze/Dry Gulch

Oak Ridge National Laboratory

Occupational Safety and Health Administration

personal computer

portable document file

Petroleum Environmental Resource Forum

Pasquill-Gifford

Passive Gaussian Plume module used with HGSYSTEM

Pasquill-Gifford/Turner

dispersion module in the SAFETI (Suite for Assessment of Fire,

Explosion, and Toxic Impact) tool kit

PLG, Inc. 
PNNL

PUFF-PLUME

Q/A

QUEST

RAPTAD

RDCA

REDIPHEM

REEDM

RF

RMP

RTVSM

RVD

SAM

SAR

SBD

SCAPA

SCIPUFF

SCRAM

SLAB

SMEDIS

SNL

SPILL/HFSPILL

SRDT

SRS

SURFACE CHEMKIN

TEEL

TIBL

TNO

TSCREEN

TSR

TTN

UDM
Pacific Northwest National Laboratory

Puff and Plume Dispersion Models for Emergency Response (computer code)

Quality Assurance

CCPS-supported aerosol generation/release code

Random Particle Transport and Diffusion (computer code)

radiological dispersion and consequence assessment

REview and DIssemination of PHysical Effects Models

model providing effluent dispersion information used with MARSS

respirable fraction

Risk Management Plan

Real Time Volume Source Dispersion Model (computer code)

Relief Valve Discharge module used with TSCREEN

Site Acquisition of Meteorology

Safety Analysis Report

safery basis documentation

Subcommittee on Consequence Assessment and Protective Actions

Second-Order Closure Integrated Puff (computer code)

Support Center for Regulatory Air Models

Slab Model (computer code)

Scientific Model Evaluation of Dense Gas Dispersion Models

Sandia National Laboratories

transient pressurized liquid release modules that are used with HGSYSTEM

Solar Radiation Delta-T

Savannah River Site

Kinetic modeling of Chemical Surface deposition (computer code)

temporary emergency expostire limits

thermal internal boundary layer

The Netherlands Office of Scientific Research

Toxic Screen (computer code)

technical safety requirements

Technical 'Transfer Network

Unitifed Dispersion Model (computer code) 
USAF

USFS

UTM

VDI

VLSTRAC

VOC

WG

WINDS

WSRC

WWW

$\chi / \mathrm{Q}$
United States Air Force

United States Forest Service

Universal Transverse Mercator

Verein Deutscher Ingenieure (computer code)

Vapor, Liquid, Solid Tracking (computer code)

volatile organic compound

Working Group

Weather Information Network Display System

Westinghouse Savannah River Company

World Wide Web

Nomalized concentration with source term 


\section{MODEL REVIEW AND EVALUATION FOR APPLICATION IN DOE SAFETY BASIS DOCUMENTATION OF CHEMICAL ACCIDENTS - MODELING GUIDANCE FOR ATMOSPHERIC DISPERSION AND CONSEQUENCE ASSESSMENT}

\section{SUMMARY}

The U.S. Department of Energy (DOE), through its Defense Programs (DP), Office of Engineering and Operations Support, established the Accident Phenomenology and Consequence (APAC) Methodology Evalwation Program to identify and evaluate methodologies and computer codes to support accident phenomenological and consequence calculations for both radiological and nonradiological materials at DOE facilities and to identify development needs. The program is also intended to define and recommend "best or good engineering/safety analysis practices" to be followed in preparing "design or beyond design basis" assessments to be included in DOE nuclear and nonnuclear facility safety documents.

The APAC effort is intended to provide scientifically sound and more consistent analytical approaches, by identifying model selection procedures and application methodologies, in order to enhance safety analysis activities throughout the DOE complex. The major objectives of this effort are to:

- Identify and assess the adequacy of models or computer codes to support calculations for accident phenomenology and consequences, both inside and outside the facility, associated with chemical and/or radiological spills, explosions, and fires;

- Provide specific guidance on the "best" or most appropriate methods and computer codes for use in safety and consequence analyses; and

- Specify dose or toxic reference levels for determining off-site and on-site radiological/toxicological exposures and health effects.

The Chemical Dispersion and Consequence Assessment (CDCA) Working Group was one of six working groups formed to carry out the mission and objectives of the APAC Methodology Evaluation Program. Consistent with the APAC objectives, a major goal of the CDCA Working Group was to establish guidance for the selection and application of chemical dispersion models and methods best suited for consequence assessment applications supporting DOE facility safety documentation. These documents include Basis for Interim Operations (BIO) reports, Justification for Continued Operation (JCO) reports, and Safety Analysis Reports (SARs). The working group consisted of seven members with broad experience in air pollution meteorology, atmospheric transport and dispersion model development and evaluation, process safety engineering/management, risk assessment, chemical accident emergency planning and response, atmospheric physics, and mathematics.

The basic strategy implemented by the CDCA Working Group was to (1) compile a comprehensive list of consequence assessment computer codes designed for air dispersion 
modeling applications, (2) screen the list to a manageable number (20 to 30 models) for review, (3) place the codes to be reviewed into two groups - one group (Tier $D$ ) receiving detailed review and evaluation (including code execution with test problems) and the other group (Tier II) receiving detailed review only - and (4) carry out those reviews and evaluations and make recommendations for code use on the basis of the resuits. The test problems for the Tier I codes were designed to be representative of chemical accident scenarios that might typically occur at DOE installations, but they were not intended to be all-inclusive of the spectrum of accident sequences that are possible. It is important to note that the scope of the current APAC program does not provide for evaluation of model performance through comparison of code predictions with field or laboratory data. Although some independent performance evaluations have been conducted for a few of the models reviewed in this study, more work in this area is needed to provide decision makers with a greater degree of confidence in consequence assessment results.

To implement its strategy, the working group identified and reviewed DOE Orders, standards, and regulatory documents to help in determining the minimum requirements for chemical dispersion and consequence assessment. It should be noted that those orders, standards, and other requirements are not written to'provide specific guidance to the safety analyst. In addition to the document review, user needs were identified through a limited survey of safety analysts within the DOE complex. This information, along with knowledge of DOE chemical inventories, helped form a foundation for selecting appropriate models for review and for deciding on the forn and substance of the modeling recommendations and guidance presented in this report.

The working group members initially compiled a list of 135 computer codes, including 7 codes with chemical transport and dispersion capabilities also recommended for review by the APAC Spills Working Group. These computer codes were identified for review and evaluation to determine their applicability with respect to chemical dispersion accident analysis at DOE facilities. The codes included both dense gas and neutrally buoyant transport and dispersion thodels. Nine requirements, or criteria, were identified for use in screening the codes for further review. The initial screening was based on the following four model requirements: (1) portability and availability in the public domain, (2) "actively maintained" by the developer or model custodian/vendor, (3) applicable to chemical releases, and (4) applied in practice. The working group requirement for access to a model's source code eliminated proprietary models from further consideration. Application of this initial screening eliminated 100 of the codes. Further refinements to the screening process reduced the number of codes under consideration from 34 to 24. This group of 24 models, which included all but 1 of the 7 codes reviewed by the Spills Working Group, was assigned to one of the two review levels (Tier I or Tier ID) on the basis of meeting all or all but one of the remaining four screening criteria. All of the remaining Spills Working Group codes were selected for Tier I review. Models supported by EPA and models with special or unique capabilities were also put in Tier I. This procedure resulted in assignment of 13 models to an in-depth Tier I review and the remainder (11) to a less comprehensive, but still detailed, Tier II review. The 11 Tier II models received the same technical review as the Tier I models but were not run on the test problems. Table S.1 lists the Tier I and II models evaluated by the working group. 
TABLES.1 Public Domain Models Reviewed by the CDCA Working Group

\begin{tabular}{|c|c|c|c|}
\hline \multicolumn{2}{|c|}{ Tier I Reviews } & \multicolumn{2}{|c|}{ Tier II Reviews } \\
\hline Code Name (Ver.) & Developer/Sponsor & Code Name (Ver.) & Developer/Sponsor \\
\hline $\operatorname{ADAM}(2.1)$ & $\begin{array}{l}\text { Phillips Lab, Hanscom } \\
\text { AFB/U.S. Air Force }\end{array}$ & AFTOX $(4.0)$ & $\begin{array}{l}\text { Phillips Lab, Hanscom } \\
\text { AFB/U.S. Air Force }\end{array}$ \\
\hline ALOHA (5.2) & NOAA, HMRAD/EPA & $\operatorname{AQPAC}^{b}(1.2 \mathrm{a})$ & EC/AES \\
\hline CALPUFF (3.0) & Earth Tech/EPA & $\begin{array}{l}\text { ARAC II } \\
\text { (CG-MATHEW/ } \\
\text { ADPIC 4.0) }\end{array}$ & LINL/DOE-DP \\
\hline CASRAM-SCa $(0.8)$ & $\begin{array}{l}\text { ANL \& University of } \\
\text { Illinois/DOT \& DOE }\end{array}$ & $\begin{array}{l}\text { CTDMPLUS } \\
(93228)\end{array}$ & $\begin{array}{l}\text { EPA (ENSR }{ }^{d} \text { and } \\
\text { Earth Tech }) / E P A\end{array}$ \\
\hline DEGADIS (2.1) & $\begin{array}{c}\text { University of } A K / C G \text {, GRI } \\
\text { and EPA }\end{array}$ & $\begin{array}{l}\text { EMGRESP } \\
(1991)\end{array}$ & ARB-OME/OME \\
\hline FEM3C (3.0) & LLNU/U.S. Anny & GAUSI $(1.0)$ & F.A. Gifford/LANL \\
\hline HGSYSTEM $(3.0)$ & Shell Research Ltd.API & HARM-II $(2.01)$ & NOAA-ARLDOE \\
\hline $\begin{array}{l}\text { HOTMAC/RAPTAD } \\
(\operatorname{Ian} 1996)^{\mathrm{a}}\end{array}$ & LANLUU.S. Air Force & MARSS (3.1) & ENSCO, InC.NASA \\
\hline INPUFF $(2.3)$ & EPA, ASRL, RTP/EPA & PUFF-PLUME & SRSIDOE \\
\hline $\begin{array}{l}\text { HASCAL } \\
\text { SCIPUFE (1.0) }\end{array}$ & ARAP/DSWA & RIVSM (3.02) & $\begin{array}{l}\text { H.E. Cramer Co/ } \\
\text { U.S. Army-Dugway }\end{array}$ \\
\hline SLAB (June 1990) & LLNL/DOE & $\begin{array}{l}\text { VDI } 3783(3.0) \\
\text { (Parts } 1 \text { and } 2)\end{array}$ & $\begin{array}{c}\text { University of Hamburg/ } \\
\text { VDI }\end{array}$ \\
\hline TSCREEN (94133) & EPA (PES, Inc) & & \\
\hline VLSTRACK $(16)$ & NSWCU.S. Navy & & \\
\hline
\end{tabular}

" Because of the lack of a user"s guide anthor final code documentation at the time of code execution with the release scenarios. model resules are not given in the discussion or the compatison plots presented in Section 5.2.2. Because the HASCAL (1.0) modeling systetn was designed for rather specialized applicutions intolving biological wapons and facilties, its tallored model input and output structure limited the evaluation and fair comparison (with obher code) of the SCIPUFF (vec. 0.338) model with the chemical relesse scerarios considered in this study. A private sector version of HOTMACRAPTAD, with the same nate, also exists and is included as an alternative "Appendix $B^{\text {"N model }}$ in the U.S. Environmental Ptotection Agency's (EPA's) Guideline on Air Quality Madeling (FR August 12, 1996).

b This model is now licensed for sole distribution through Environmental Sofware Consultants as a proprietary model.

c The decision to assign this model to Tier II review was based primarity on the issue of code porability outside Lawrence Livernore National Laboratory (LLNL). Considering the Incicipated conlinued DOE lunding suppon of LLNL's Amospheric Releasc Advisory Capability. including the gentrocion of the ARAC Ill systern of models, the CDCA Working Group recommends that ARAC be considered for any subsequent mode! reviews and/or evaluaions. A complete veview. of course, would depend on salisfactory resolution of the issues peraining to the FORTRAN soune code avalability and to medel porability.

d Formerly ERT, inc.

e Formerly Sigma Research, Inc. 
The scientific evaluations of the Tier I and II computer models were designed to address not only generic issues common to each code, but also specific technical capabilities of the codes (e.g., passive versus heavy gas dispersion, atypical plume behavior). For each Tier I model, one reviewer was selected from among working group members to conduct the evaluation exercise, including running the model on the test problems (except the DEGADIS review, which was split between two working group members). To avoid conflicts of interest, no reviewer was assigned to evaluate a model that he or she had helped develop. Although model developers were not directly involved in the reviews, they were consulted as needed to clarify technical issues and to assist in the quality assurance of the model setup and input data assumptions for each of the test scenarios and the model review sheets. The overall evaluation of a code (i.e., general, technical, and sample problem) took into consideration its ability to support dispersion and consequence calculations for a spectrum of chemical releases that might be encountered. Subsequent to completion of the Tier I reviews, the individual model results were made available to the developer of that model for review and comment.

The CDCA Working Group conducted its deliberations over a 14-month period. Three meetings and a series of conference calls were held during that period. The initial meeting was held on July 13, 1995, to discuss the scope and mission of the working group and to begin screening the initial list of codes. The second meeting, which focused on establishing the details of the two-tiered approach for the model reviews, was held before the International Conference and Workshop on Modeling and Mitigating the Consequences of Accidental Releases of Hazardous Materials, held in New Orleans, Louisiana, September 26-27, 1995. Most of the modei reviews/evaluations were conducted from February to September 1996. During that period, monthly conference calls were held by working group members to discuss status and modeling issues. The last meeting of the working group was September 26-27, 1996, at Argonne National Laboratory (AVL) to (1) review the strengths and weaknesses of each Tier I and II model, (2) discuss the model test results, (3) determine the applicability of each model relative to DOE hazard and analysis class, and (4) resolve modeling issues. Additional conference calls and several follow-up consultations among working group members were held to help reach consensus on remaining issues and on the working group's recommendations.

On the basis of the model reviews, the Tier I test problem results, and the expert judgment of the working group members, it was concluded that no one model could be recommended to best handle the diversity of all possible facility accident conditions and release environments that could occur within the DOE complex. It was also the working group's collective judgment that there was no objective way to rank or rate the reviewed models or their specific attributes without the aid of a combined comprehensive model performance evaluation and sensitivity analysis. Therefore, the working group focused its recommendations on a halfdozen key elements of its model reviews, including outcome of the test problem results. Although not explicitly accounted for, the recommendations also considered individual model capabilities for use in program- and project-specific safety analysis classes and facility hazard categories. Each Tier I model was eithcr conditionally recommended for generally broad or special limited safety basis documentation (SBD) applications within its capability domain, recommended for further consideration and review, or not recommended. Each Tier II model was either conditionally recommended for further consideration or not recommended. Table S.2 lists the CDAC conditionally recommended models. This table or any other table of models contained in 
this repor is not intended to imply any relative ranking (e.g., "best" to "worst") among the models reviewed by the working group. These recommendations were based on several factors, including consideration of a model's limitations. strengths and weaknesses; previous model performance results; and for (Tier D results of the test problems. In addition, the working group supplemented its code recommendations with a set of recommended "best practices" (e.g., good engineering, modeling, and safety analysis practices) for modeling the transport, dispersion, and consequences of chemical plumes released to the atmosphere. These practices included specific analysis and documentation procedures, including guidance on determining conditions for assessing design basis incident ("worst-case") consequences. The best practices and the specific model recommendations are intended to provide the safety analyst with scientifically sound procedures and methods for conducting consistent chemical consequence accident assessments.

In its frnal deliberations, the CDCA Working Group identified and discussed several needs of chemical accident safety analysts. These needs include improving, identifying, or developing refined models for atmospheric dispersion simulations, including performance evaluation, and establishing a support network to answer technical questions, exchange ideas, and provide training. Filling these needs would provide the safety analyst with additional tools to

TABLE S.2 CDCA Conditional Model Recommendations

\begin{tabular}{ll}
$\begin{array}{c}\text { Applicable to Generally } \\
\text { Broad SBD Applications }\end{array}$ & $\begin{array}{c}\text { Applicable to Generaliy Limited } \\
\text { or Special SBD Applications }\end{array}$ \\
\hline ALOHA & $\begin{array}{l}\text { ADAM } \\
\text { CALGADIS }\end{array}$ \\
HGSYSTEM & FEM3C \\
SLAB & INPUFF \\
& TSCREEN \\
\hline Consideration for Further Review and Evaluation \\
\hline
\end{tabular}

a Tier I models that could have generally broad or special SBD applications and that were under development and not fully documented during the working group reviews. With the exception of CASRAM, these models have been published in the peer-revicwed literature and are well known in the air dispersion modeling community. SCIPUFF and HOTMAC/RAPTAD (proprietary version) are established, widely used modets whose latest versions are still in the process of being tested and/or furher developed. 
improve the accuracy of assessments and promote consistency in safety analyses across the DOE complex. The recommendations to address these needs are summarized as follows:

- Expand and refine models included in Tier I reviews and evaluations -. Supplement the Tier I model list with additional codes for evaluation with test problems. This list would include some Tier I and II codes that merited further evaluation and other codes not specifically identified that would wartant Tier I consideration. Proprietary codes, including new or overlooked models, as well as those on the initial list of 135 codes considered by the working group, could also be considered or reconsidered for review.

- Conduct a systematic model performance evaluation - To ascertain the accuracy of and uncertainty in model estimates, a quantitative model performance evaluation with a standard set of acceptable statistical performance measures and field data should be conducted for a select group of codes. Field data for conducting the evaluations could include the Burro (liquefied natural gas), Maplin'Sands (liquefied natural gas), Desert Tortoise (ammonia), Goldfish (hydrogen fluoride), and Thomey Island (Freon) data sets. The recent Kit Fox dense gas experiments are highly recommended for use since they emphasize finite duration releases over rough surfaces during stable meteorological conditions. These conditions are precisely the key data that have been lacking in previous model performance evaluations. Other relevant dense gas field and laboratory experimental data, including the European Union funded Review and Dissemination of Physical Effects Models (REDIPHEM) database and data currently being considered for use by the European-based Scientific Model Evaluation of Dense Gas Dispersion Models (SMEDIS) Programme (coordinated by the Health and Safety Executive of the United Kingdom), could also be considered.

- Address model development needs - The working group identified several analysis or capability issues that were not adequately addressed by the reviewed public domain models. Weaknesses in existing models could be addressed through a targeted model upgrade program or through further model development, including incorporation of state-of-the-science features not present in any one code. Some of the key areas needing improvement include accounting for (1) increased surface roughness typically associated with industrial plant sites and many DOE installation sites; (2) short-duration releases; (3) aerodynamic effects of buildings and surrounding terrain on near-surface releases; (4) removal mechanisms, including wet and dry deposition; and (5) aerosol fornation and evaporation. Although not identified or discussed in the model development needs, treatment of exposure and toxic load effects will continue to be an important area for further research and development.

- Establish a safety analysis clearinghouse Web page - An accident consequence/safety anaiysis (ACSA) Home Page on the World Wide Web is 
recommended to serve as a clearinghouse to respond to questions raised by safety analysts and to provide pertinent guidance on the selection and application of chemical accident dispersion models in support of SARs, BIOs, and JCOs. "Chat sessions" could also be implemented on the Web site for discussion of common problems encountered by users in the setup and execution of models conditionally recommended in this report. Although the Home Page would be established to serve the modeling community under the APAC umbrella, broader participation from national and intemational organizations involved in chemical and radiological accident consequence assessment and accident prevention could evolve. A prototype of what the CDAC component of the ACSA home page might look like is presented in the recommendations of this report.

- Conduct chemical dispersion modeling workshops - A series of workshops could be conducted to train safety analysts in the skills required to perform consistent and credible safety analyses for potential accidents involving the release, transport, and dispersion of hazardous chemical compounds. The workshops would provide for hands-on modeling training, with selected models run on workshop PCs for a full spectrum of accident scenarios.

- Establish a joint Spills and CDCA Working Group -- The test problems run for the Tier I models illustrated the difficulty of separating chemical spills and dispersion modeling calculations in many of the codes that incorporated both. If DOE chooses to implement any of the recommendations listed above from the CDCA Working Group and/or the recommendations from the Spills Working Group, an efficient and cost-effective means to address some of the cross-cutting issues would be by establishing a consolidated Spills and CDCA Working Group or a coordinated working group effort. 


\section{INTRODUCTION}

The U.S. Department of Energy (DOE) operates numerous production, reprocessing, and storage facilities that handle hazardous materials. DOE also operates hazardous waste management facilities located at national laboratories and other DOE installations and field offices throughout the United States. These DOE facilities are subject to the safety oversight of external (i.e., the Defense Nuclear Facilities Safety Board [DNFSB]) and internal (e.g., DOE Office of Engineering and Operations Support) entities to ensure that the level of hazard is appropriately assessed, that operational and shutdown risks are within prescribed limits, that voluntary worker and involuntary general public risks are tolerable, and that development of emergency action procedures is commensurate with the level of hazard posed.

To demonstrate a technically defensible basis for preparation of Safety Analysis Reports (SARs), Basis for Interim Operations (BIO) reports, and Justification for Continuing Operations ( $\mathrm{SCO}$ ) reports (collective referred to as safety basis documentation, or SBD), hazard screenings and analyses have been performed to bring nonreactor nuclear facility safety documentation into compliance with DOE Orders and standards. The content and specification requirements of accident analyses performed for a facility, including hazard analysis, source term estimates, accident sequence progression, and the ensuing consequence analysis, are now more rigorous than previously required. More detailed studies are now being routinely required, including accident phenomenology and consequence analysis.

Chemical transport and dispersion models have been developed to assist in facility safety analysis, emergency planning, environmental impact assessment, and other analytical needs within the DOE complex and elsewhere. A large portion of the public domain model development has taken place at the national laboratories and major DOE installations. Other federal government agencies also contributing to this development include the U.S. Environmental Protection Agency (EPA), the U.S. Coast Guard, the National Oceanic and Atmospheric Administration (NOAA), the U.S. Department of Defense (DOD), the U.S. Department of Transportation (DOT), and the National Aeronautics and Space Administration (NASA). Software has also been developed by scientists and engineers within the chemical and petrochemical industries and by consultants to this industrial group. In many cases, there has been parallel development of software for the same purpose, but supported by different funding sources. As expected, many of these computer codes exhibit varying capabilities associated with inherent limitations and specific technical strengths and weaknesses. In general, however, despite the number of codes of varying capabilities available, little guidance is provided regarding (1) the selection and application of chemical dispersion models for consequence assessment specific to various DOE hazard categories, input assumptions, and data, or (2) the level of modeling sophistication required at the facility at which the code is to be applied.

To address the current software and analysis issues facing safety analysts at DOF. facilities, the DOE Accident Phenomenology and Consequence (APAC) Methodology Evaluation Program was established at the request of the Office of Defense Programs (DP). The objective of this program is to review and evaluate the analysts' approaches (selection and application of computer codes and model/modeling assumptions) applied to support SAR, BIO, 
and hazard analysis documentation and to recommend specific models and standard practices to improve the technical quality and consistency of safety analysis documentation. In particular, this report provides guidance on sound technical approaches for atmospheric dispersion modeling of accidental chemical releases to aid in the preparation and review of consistent SAR documentation.

The remainder of this chapter describes the purpose and objectives of the APAC initiative. It also highlights the relevant DOE Orders, standards, and other requirements dictating the application of atmospheric dispersion computations and accident analyses in safety analysis reports and discusses a survey of current safety analysis practices within the DOE complex. To familiarize the analyst with some of the physics associated with the assessments, a description of some of the fundamental dispersion modeling principles of importance to safety analysis work is provided in Chapter 2 . Chapter 3 summarizes the safety analyst survey, identifies the three-step process used by the Chemical Dispersion and Consequence Assessment (CDCA) Working Group to select the models to be reviewed, and summarizes each of the codes selected. The bases used in the model reviews and a description of the accident scenarios used in the detailed model reviews to which select codes were subjected are detailed in Chapter 4 . The results of the model reviews and evaluations and the working group recommendations are presented and discussed in Chapters 5 and 6 , respectively. The appendixes to this report provide supplemental and complementary information on the regulatory requirements, chemical inventories, health criteria for defining hazard zones, and the model reviews.*

\subsection{PURPOSE}

The APAC Methodology Evaluation Program is a Defense Programs activity intended to provide guidance for improving the quality and consistency of the methods of analysis used for SARs, BIO reports, JCO reports, and hazard analysis documents. The objectives of this effort are as follows:

- Define current analysis practices and needs of safety analysts and identify requirements reflected in DOE Orders, standards, and guides for safety analysis.

- Identify and recommend methods (e.g., hand calculations and computer codes) available for use by safety analysts in assessing transpor and dispersion of contaminants and associated consequences (e.g., acute exposure health effects) to workers and members of the public.

- Evaluate the status and adequacy (detailing both strengths and limitations) of selected models and computer codes available for conducting accident phenomenology and consequence analyses.

\footnotetext{
Because of size constraints, the appendixes have not been printed with the document; however, copies of individual appendixes cited in the text may be obtained upon request from Michael Lazaro. Environmental Assesssment Division, Argonne National Laboratory, 9700 S. Cass Avenue, Argonne, Illinois.
} 
- Define good engineering/safety analysis practices, or "best practices," to be followed in the analyses supporting facility safety docurnentation.

- Provide recommendations to DOE on cost-effective approaches to meet APAC program objectives.

The APAC activity is overseen by an executive committee, with the work being carried out by six working groups. The working groups have reviewed selected computer codes in the following areas of accident phenomenology:

- Fire Analysis

- Explosions and Energetic Events (EEE)

- Spills Analysis

- In-Facility Release and Transport Analysis

- Radiological Dispersion and Consequences Assessment (RDCA)

- Chemical Dispersion and Consequences Assessment (CDCA)

The Spills and Fire Working Groups have focused on methods for characterizing and quantifying atmospheric releases. The scope of the Fire Working Group also addressed dispersion parameters for characterizing releases to the atmosphere from combustion products escaping building confinement or exterior fires. Although source term characterization was included in the EEE working group deliberations, its primary focus has been on methods to characterize explosion physics and effects. The focus of the last two working groups has been on methods to simulate transport and dispersion of contaminants subsequent to accidental release and the human health effects from exposure to these releases. The In-Facility Working Group was the only working group dealing with releases within a facility and charged with both source term and transport and dispersion characterization.

This report documents the work and recommendations of the CDCA Working Group. The affiliations, addresses and telephone numbers of the seven working group members are listed in Table 1.1.

Additional support was obtained from J. Chang of Earth Technology, Inc., and H. Wang of Argonne National Laboratory, who assisted in running some of the Tier I test problems. Model developer comments on reviews of their models and the test problem results were factored into the working group's findings. Independent peer review of this report was provided by the EPA, the Vapor Cloud Dispersion Subcommittee of the Center for Chemical Process Safety (CCPS) of the American Institute of Chemical Engineers (AIChE), and a European community research group currently operating under the Scientific Model Evaluation of Dense Gas Dispersion (SMEDIS) Models research program. 
TABLE 1.1 CDCA Working Group Members

\begin{tabular}{|c|c|}
\hline Michael Lazaro & Phone: (630) 252-3447 \\
\hline Argonne National Laboratory & Fax: (630) $252-3194$ \\
\hline 9700 South Cass Avenue & E-mail: muazaro@anl.gov \\
\hline \multicolumn{2}{|l|}{ Argonne, IL 60439} \\
\hline David Hesse & Phone: (614) 424-7519 \\
\hline Battelle Columbus Laboratories & Fax: (614) 424-3404 \\
\hline 505 King Avenue & E-mail: hesse@battelle.org \\
\hline \multicolumn{2}{|l|}{ Columbus, $\mathrm{OH} 43201$} \\
\hline Steve Hanna & Phone: (508) 263-6095 \\
\hline Earth Tech & Fax: $(508) 263-6700$ \\
\hline 196 Baker Avenue & E-mail: Ibhanna@ma.ultranet.com \\
\hline \multicolumn{2}{|l|}{ Concord, MA 01742-2167 } \\
\hline Keith Woodard/Jackie Lewis & Phone: (301) 907-9100 \\
\hline PLG, Incorporated & Fax: (301) 907-0050 \\
\hline 7315 Wisconsin Avenue & E-mail: kwoodard@plg.com \\
\hline Suite 620 East & or \\
\hline Bechesda, MD 20814 & jlewis@plg.com \\
\hline Carl Mazzola & Phone: (706) 650-0939 \\
\hline Stone \& Webster Engineering & Fax: $(706) 650-0887$ \\
\hline 4163 Hammond's Ferry Road & E-mail:carl.mazzola@stoneweb.com \\
\hline \multirow{2}{*}{\multicolumn{2}{|c|}{$\begin{array}{l}\text { Suite M } \\
\text { Evans. CA } 30809\end{array}$}} \\
\hline & \\
\hline Terry Huang & Phone: (803) 952-6673 \\
\hline Westinghouse Savannah River Co. & Fax: (803) 952-8305 \\
\hline $730-2 B$ & E-mail: ju-chrong.huang@srs.gov \\
\hline Aiken, SC 29808 & \\
\hline
\end{tabular}

\subsection{OBJECTIVE AND SCOPE}

The primary objective of the APAC CDCA Working Group has been to identify and evaluate publicly available computer codes with broad or special capabilities for conducting chemical-dispersion, hazard-related analyses in support of DOE safety basis documentation (SBD). In addition, the working group seeks to (1) delineate a set of recommended practices or assumptions for use when conducting hazard analyses associated with a chemical plume or puff, and (2) identify future development needs in the area of chemical consequence assessment. The 
overall process followed by the CDCA Working Group to accomplish these objectives is depicted in Figure 1.1 and briefly described below. The Tier I and Tier $I$ boxes in the figure refer to detailed model reviews and evaluations with (Tier $D$ ) and without (Tier II) code execution with test problems.

\subsubsection{Review Regulatory Requirements}

DOE and other applicable federal regulatory and guidance documents were reviewed to identify requirements pertaining to air dispersion modeling and consequence assessment. AIthough the requirements for vapor dispersion analysis generally are not specific, an attempt was made to interpret them as a specific basis for judging the capabilities of each code.

\subsubsection{Identify and Select Computer Codes Applicable to APAC Chemical Dispersion and Consequence Assessment}

An early step in the process was compiling a list of both proprietary and publicly available atmospheric transport and dispersion models. After some deliberation, working group members decided to consider only public domain codes as potential candidates for evaluation. The identified computer codes and their support documentation were then obtained for general and technical review and evaluation.

Proprietary codes were excluded from consideration in this study primarily because of the lack of accessibility to the source code. For most proprietary models, a source code is not available for independent inspection, nor are the full theoretical details and assumptions in the model algorithms published for public dissemination. However, the working group recognized that in select areas of dispersion modeling, proprietary codes may offer modeling capabilities not available in the public domain. Accordingly, proprietary codes may be revisited at a later time.

\subsubsection{Develop Comprehensive Basis for Model Reviews}

Besides the review and comparison of model sample problem results, a basis for evaluating the capabilities of each computer code was also developed. The basis included review and evaluation of the general and specific technical capabilities and limitations of each code and of its adequacy in the context of a graded modeling approach. A description of the model review and evaluation basis is provided in Section 4.2 .

\subsubsection{Develop Sample Problems for Tier I Reviews}

A set of seven sample problems was developed to test the capabilities of the Tier I codes. The sample problems represent a wide range, both in type and in magnitude, of chemical accident release scenarios required to address a variety of air dispersion conditions. These scenarios were extracted from chemical accident problems used in the American Institute of Chemical Engineers (AIChE) Guidelines for Use of Vapor Cloud Dispersion Models (AIChE/CCPS 1996). The sample problems are described in Section 4.3. It should be noted that five of the release scenarios depicted in the sample problems were primarily designed for dense gas simulations. 


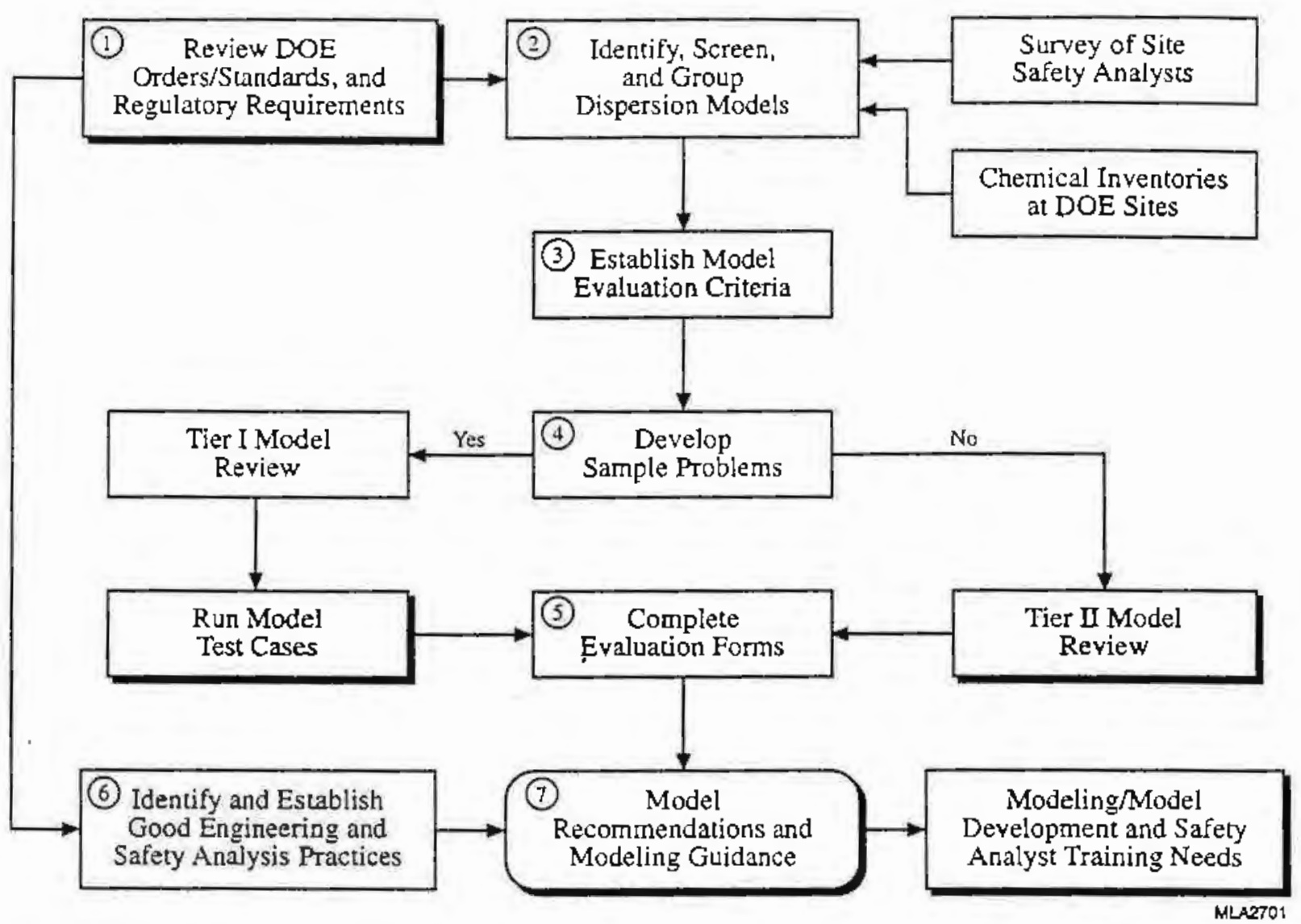

FIGURE 1.1 Model Review/Evaluation Framework for the CDCA Working Group

\subsubsection{Evaluate Computer Models (Tiers I and II) and Compare Test Case Results (Tier I)}

After data were collected and sample problems developed, the working group focused on evaluating the availability, cument status, and technical adequacy of computer models used to perform vapor dispersion analyses. Specific modeling capabiiities of each Tier I and Tier II code (see Section 3.2) were judged against the basis for evaluation, along with insights about code strengths and weaknesses. Results of the Tier I Model sample problems are reviewed, compared, and reported (see Section 5.2).

\subsubsection{Identify Best Practices}

During the evaluation of the computer models, a list of "best practices" that describe well-regarded chemical dispersion modeling analysis methods and tools for safety analysts was compiled. Best practices include selection of model input parameters for conservative and the more likely or probable consequence assessments, computational assumptions and procedures, simplification schemes, and advanced or undocumented code usage techniques. 


\subsubsection{Develop Conclusions and Recommendations}

As the evaluations were performed. Final conclusions were drawn by comparing the scientific evaluations and the results of the sample problem exercise where applicable. In addition, a set of "best practices" was documented and recommendations were made. This information is presented in Sections 6.1 and 6.2. The model recommendations for conducting a vapor dispersion and consequence analysis are presented in Section 6.3.

\subsubsection{Identify Needs for Improving Consequence Assessment for SBD}

On the basis of the model reviews and evaluations conducted by the CDCA Working Group and information collected during the survey of site safety analysts (see discussion in Sections 1.4 and 3.1), key model development and model application training needs were identified. These needs included recommendations for model performance evaluation, further Tier I model reviews, and identification of specific areas for model improvement.

\subsection{DOE GUIDING ORDERS AND OTHER FEDERAL REGULATORY DOCUMENTS}

DOE Orders on accident analysis are intended to ensure determination of the health and safety risk at a DOE facility and, if necessary, what mitigation factors are needed to minimize human health and environmental impacts. In general, DOE Orders cover regulatory requirements, but extend further into risk reduction. A more detailed discussion of the applicable DOE Orders, guides, and handbooks is presented in Appendix A.'

The initial step in the working group's process was to review present and past DOE regulatory and licensing documents and to identify specific requirements that would influence the type of analyses considered acceptable for safety analysis. The identified requirements and capabilities were further developed into the basis for evaluation of chemical dispersion methodologies. The CDCA Working Group reviewed the following documents:

- Facility Safety Analysis Reports and Hazard Analyses

- DOE Order 5481.1B, Safety Analysis and Review System

- DOE-STD-1027-92, Hazard Categorization and Accident Analysis Techniques for Compliance with DOE Order 5480. 23, Nuclear Safety Analysis Reports

- DOE-STD-3009-94, Preparation Guide for U.S. Department of Entergy Nonreactor Nuclear Facility Safety Analysis Reports

1 Because of size consiraints, appendixes prepared in support of this report have not been printed with the document: however, copies of individual appendixes cited in the text may be obtained upon request from Michael Lazaro, Environmental Assessment Division, Argonne National Laborarory, 9700 S. Cass Avenue, Argonne, Illinois. 
- DOE-STD-3011-94, Guidance for Preparation of DOE 5480.22 (TSR) and DOE 5480.23 (SAR) Implementation Plans

- Process Safety and Risk Management

- 29 CFR 1910.119, OSHA Worker Chemical Safety Process Safety Management

- 40 CFR 68, EPA Risk Management Programs for Chemical Accident Release Prevention

- Emergency Planning

- DOE Order 151.1, Planning and Preparedness for Operational Emergencies

- Design Basis Requirements

- DOE Order 6430.1A, General Design Criteria

\section{- DOE Handbooks}

- DOE-HBK-3010-94, Recommended Values and Technical Bases for Airborne Release Fractions (ARFs), Airborne Release Rates (ARRs), and Respirable Fractions (RFs) at DOE Non-Reactor Nuclear Facilities.

The approach taken was to review each of these documents and identify relevant sections. An attempt was then made to interpret the citations from each document as requirements for chemical dispersion analysis. These interpretations were summarized, and an overall set of requirements was identified. The requirements were then developed into computer code evaluation bases, which are summarized in Section 4.

Given that the DOE regulatory environment is frequently changing, and new orders and rules are being continuously developed and issued, future requirements for safety analysis may differ from those that are followed today. It is therefore prudent to consider revisiting the information in this report with respect to requirements at a future date.

\subsection{CURRENT CHEMICAL SAFETY ANALYSIS PRACTICES WITHIN THE DOE COMPLEX}

A survey of safety analysts and emergency management personnel was conducted to identify accidents analyzed and models applied in past chemical hazard SARs that have been developed at each DOE facility. A series of 12 questions, focused on chemical accident consequences, was posed to various safety analysts and emergency management personnel at major DOE facilities in order to gain insight into chemical accident assessment and the hazards contained at each of these facilities. In addition, chemical dispersion models of various levels of 
sophistication and robustness were identified for both SAR and emergency management use. The survey revealed a wide spectrum of situations and approaches, with some differences in the strategies employed to develop consequence assessments. The detailed results of the survey are presented in Appendix B.

Accident scenarios varied somewhat at each site because of differences in the hazardous materials inventory, materials storage and handling safeguards, and the type of operations. However, some commonality was found in the types of chemicals considered and the accident sequences employed to establish impact criteria. For example, all DOE reservations/field offices have to deal with the possibility of a release of chlorine from 1-ton or 150-pound containers at wastewater treatment facilities. The only exception is when chlorine tanks or cylinders are replaced with solid sodium hypochlorite. In addition, various sulfuric acid, hydrochloric acid, and nitric acid accident releases were hypothesized at most DOE sites, which use these very strong acids in many different processes. Similarly, accident sequences involving caustic bases, such as sodium hydroxide, underwent evaluation. Another commonly evaluated scenario was the release of ammonia, either in anhydrous form or as a refrigerated liquid. Less common sequences are considered at locations that employ unique processes. For example, at the Savannah River Site (SRS), accident sequences involving benzene and sodium tetraphenylborate are evaluated because of the unique in-tank precipitation process located only at SRS. At several of the DOE reservations/field offices, accident sequences involving a large number of volatile organic compounds (VOCs) are evaluated, since the 1990 Clean Air Act Amendments have identified about 140 VOCs as acutely hazardous air pollutants. A large majority of these accident sequences involve spills. However, for the same chemicals, sequences involving fires, deflagrations, and detonations have also recently undergone evaluation.

Until fairly recently (over the past 3 to 5 years), very few chemical hazards were evaluated at DOE sites because the focus was mainly on radiological hazards. More attention is now being given to chemical hazard assessment. The significant chemical hazards appear to be spills of chlorine, refrigerated ammonia, sulfuric acid, nitric acid, hydrochloric acid, sodium hydroxide, and many VOCs. Simple asphyxiants, which are relatively benign chemicals except when present in sufficiently large concentrations to reduce the atmospheric oxygen content below the level that can sustain life, are also considered. These chemicals include nitrogen, carbon dioxide, argon, and sulfur hexafluoride. Some DOE sites look at chemical interactions, or synergistic effects of mixing two chemicals together to yield a larger combined hazard. The mixes considered include formaldehyde-nitric acid, and sodium tetraphenylborate-oxalic acid, Still other chemicals undergo transformation in the atmosphere upon release, creating chemical by-products that are also hazardous (e.g., uranium hexafluoride at Oak Ridge). Each of the major DOE reservations/field offices has a diverse inventory of chemical hazards. In addition to many of the aforementioned chemicals, other hazards include acrylonitrile, fluorine, polychlorinated biphenyls, and phosphorus pentoxide. Appendix $\mathrm{C}$ provides a representative list of chemicals being used at DOE sites.

In direct response to the lack of detailed guidance in DOE Order 5481.1B, a variety of chemical dispersion models are applied at DOE reservations and field offices. At some DOE sites, radiological modeIs are utilized to obtain ratios of estimated concentrations to release quantities ( $\chi / Q$ values) downwind and then are integrated with hand-calculated chemical source 
term values. At other DOE locations, a variety of models are applied for safety analysis planning. Depending on the complexity of the evaluation, the following models have been applied: SLAB, DEGADIS, HGSYSTEM/UF, ALOHA, AFTOX, ARCHIE, and INPUFF. Models applied to emergency response situations involving chemical accidents include EPICODE, HARM- $\Pi$, HOTSPOT, and ARAC.

The survey uncovered a wide divergence of approaches used to perform analyses necessary to address chemical hazards in SARs. Analyses performed ranged from relatively simple hand calculations of $\chi / Q$ to the very complex treatment of atmospheric chemistry associated with spills of uranium hexafluoride. Models of varying pedigree and robustness have been applied because of the lack of a standard set of "best practices" applicable to the broad spectrum of chemical accident scenarios encountered in recent and past safety analysis work. 


\section{SELECTED SOURCE RELEASE AND AIR DISPERSION MODELING PRINCIPLES RELEVANT TO CHEMICAL CONSEQUENCE ASSESSMENT}

This chapter is intended to provide a summary of fundamental principles important in applying and interpreting dispersion modeling results. The discussion covers four basic areas relevant to modeling and understanding vapor cloud dispersion and exposure consequences. These areas include influences of release physics/thermodynamics, influences of meteorology, the role of hand calculations in safety analysis, and considerations important in accounting for the effects of release duration and travel, averaging, sampling, and exposure times.

It is important to note that this discussion is not intended to cover all of the details necessary to achieve a comprehensive understanding of each of the areas important to the fundamentals of vapor cloud dispersion modeling and consequence analysis. The scope of this chapter is primarily limited to a discussion of the interplay between the physics of dispersion and the modeling assumptions and approaches typically employed that will allow the reader to understand the specific model evaluation criteria and their relevance to the proper selection of a dispersion code or method for analysis of a hazardous chemical release. Although numerous papers, books, and reports have been published on this topic, the reader is referred to recent and comprehensive discussions of the necessary principles such as: the Center for Chemical Process Safety's Guidelines for the Use of Vapor Cloud Dispersion Models (AlChE/CCPS 1996), the American Petroleum Institute's A Guidance Manual for Modeling Hypothetical Accidental Releases to the Atmosphere (API 1996), and the U.S. Environmental Protection Agency's Guidance on the Application of Refined Dispersion Models for Hazardous/Toxic Air Releases (EPA 1993b). A third general purpose reference, perhaps not as comprehensive, describing modeling techniques for liquid-vapor source-term and dispersion analysis is the "Yellow Book" (TNO 1992). Further references are provided in the discussion that follows.

\subsection{INFLUENCE OF RELEASE PHYSICS AND THERMODYNAMICS, AND SUBSTANCE-MIXTURE PHYSICAL PROPERTY EFFECTS}

The physical and chemical processes under which a mass of airborne vapor undergoes dispersion into the ambient environment are inextricably linked to the characteristics of the vapor source. In some cases, the relationship between the source term and the dispersion of a vapor can become highly complex. The types of source releases that evolve into complex flow fields generally consist of superheated liquid releases, two-phase emissions, high-momentum liquid and vapor jets, positively buoyant plumes, and chemicals with a high molecular weight in comparison to that of air. In contrast to the study of many physical phenomena where highly complex behavior represents the exception to the rule, complicated dispersion scenarios actually portray the typical release situation. Even in the instance of the simplest release possible - a neutrally buoyant release where the contaminant acts as a passive tracer with no induced density or temperature gradients -- the chemical transport can be complicated by the presence of vegetative canopy, buildings, rough terrain, and land-sea effects.

The objective of this section is to provide the necessary background and guidance on the effects of the physical and chemical properties of a hazardous release on its subsequent 
dispersion in the atmosphere to assist the analyst in the selection of an appropriate dispersion model for the given vapor source term. To this end, the flow diagram provided in Figure 2.1 illustrates the linkages between source term and dispersion phenomena for both instantaneous and continuous release and serves as a template for discussions throughout this section. The relevant distinctions between the dispersion characteristics of continuous versus instantaneous hazardous chemical plumes and puffs are highlighted in Section 2.4.5. The release physics of a vapor emission is classified here on the basis of continuous versus instantaneous only because certain types of releases possible in the case of a continuous source term cannot appear in an instantaneous setting because of the limited or finite vapor source duration.

Characterization of the initial vapor source term is covered in more detail in the APAC Spills Working Group report (Brereton et. al. 1995). There may also be specific elements of the source term calculation that should be addressed in the dispersion analysis. For instance, the initial characterization of liquid aerosol formation (e.g., droplet diameter, initial trajectory of flow) falls within the scope of the source term analysis. However, other facets of the vapor source term (e.g., aerosol evaporation rate, fraction of aerosol component that falls to the ground prior to complete evaporation) would ideally be treated as an integral part of the dispersion phenomena because the conditions under which transport takes place in turn affect the total vapor source term. Of course, a simplifying assumption would be to assume that all aerosol vaporizes at the source point - an assumption that would certainly be worst-case but might not necessarily be credible and that could lead to consequences of unrealistically large magnitude.

\subsubsection{Subcooled Leaks/Spills and Liquid Jets}

In most instances, a nonpressurized subcooled liquid leak or spill (i.e., a spill where the temperature of the liquid is below the saturation temperature for the existing storage pressure) simply forms a pool on the ground surface (see "Source A", Figure 2.1). In the case of a volatile liquid, there will be vapor emissions into the atmosphere associated with pool evaporation where the latent heat of vaporization is supplied by the thermal ambiance. Under most conditions, there will be no flashing of liquid to vapor upon release to ambient conditions unless the saturation pressure is much less than that of ambient pressure (such as in the case of anhydrous ammonia, for instance). Therefore, a suspended aerosol component is usually not a consideration in these types of releases. On the other hand, the vast majority of cases involving evaporating liquid pools lead to heavier-than-air vapor emissions. Therefore, it is essential that if a dispersion model is to accurately predict the dispersion of vapors from evaporating pools, it should possess dense gas modeling capabilities.

Under high-pressure release conditions, a subcooled pressurized liquid jet may undergo breakup, with a portion of the liquid emission possibly forming small liquid droplets. Jet breakup results from mechanical nonequilibrium arising from shear flow and turbulent mixing at the boundary between the jet wall and the ambient air. A number of criteria exist for determining which breakup mode (e.g., capillary vs, aerodynamic) is applicable to the release under consideration. 


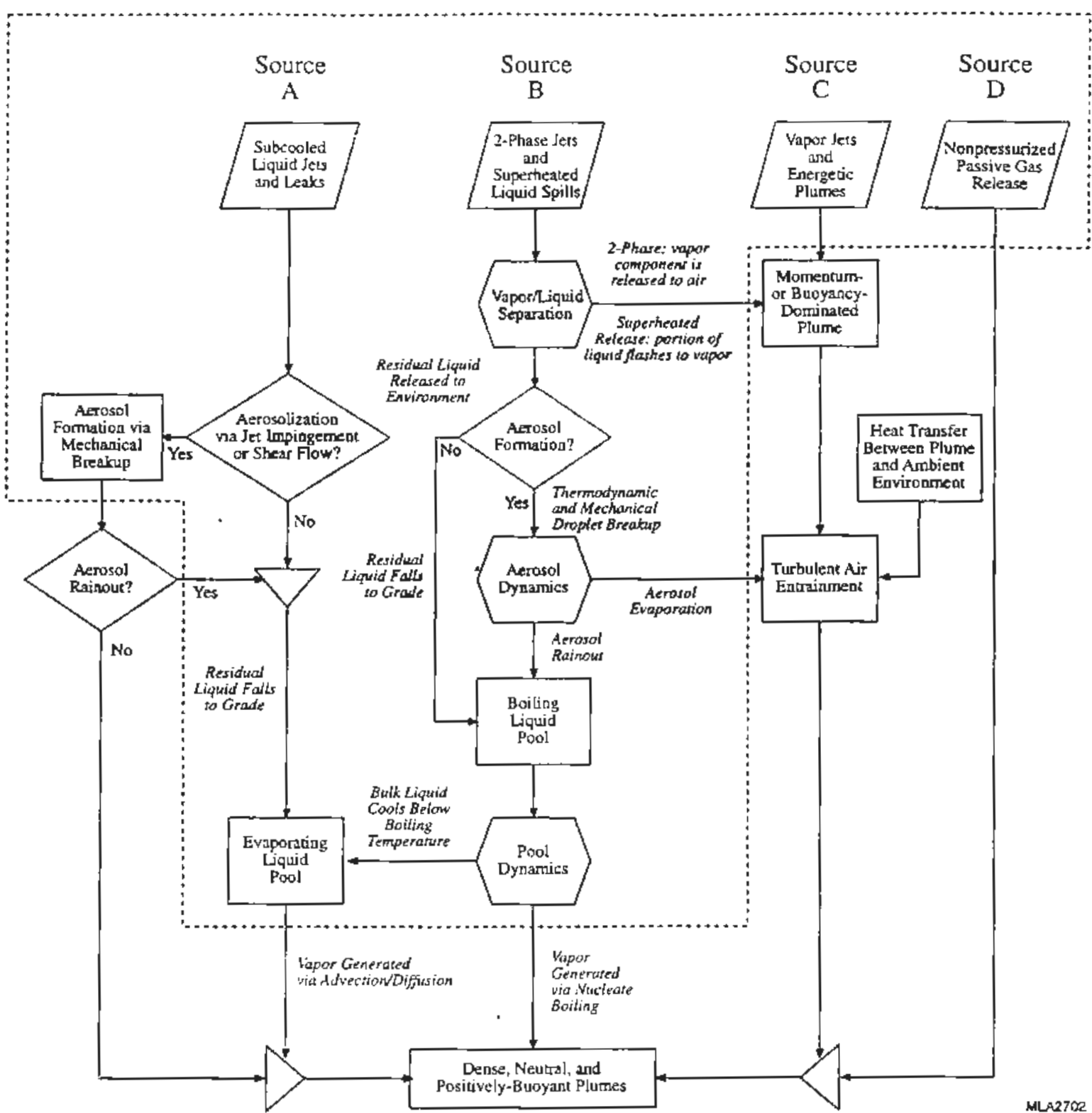

FIGURE 2.1 Phenomenological Progression for Short- (puffs, $T_{d}<0.6 T_{t}$ ) and Long(plumes, $T_{d}>2.5 T_{t}$ ) Duration Releases (See discussion in Section 2.4.5.) 
Once the flow regime has been established, a number of models may be used to calculate the droplet sizes. ${ }^{2}$ The droplet size and trajectory, as well as the prevailing wind conditions, may be used to determine if the droplet will rain out onto the ground or remain suspended as an evaporating aerosol component. Droplets that fall to the ground before complete evaporation will form an evaporating liquid pool on the ground substrate.

Subcooled liquid jet breakup leading to a suspended liquid aerosol component is generally an important factor in dispersion analysis only when either (1) the jet diameters are relatively small or (2) direct jet impingement onto an object induces large mechanical shear and breakup forces, which in turn result in an appreciable aerosol fraction. Even in the event of aerosol formation, however, most of the suspended droplets rapidly fall to the ground because of the low evaporation rate and the low convective heat transfer rate between the air and the liquid phase contaminant.

\subsubsection{Superheated Liquid Jets/Spills and Two-Phase Releases}

In contrast to the breakup of a subcóoled liquid jet, the rapid expansion of a superheated liquid (a liquid at a temperature exceeding the saturated-vapor temperature at the given pressure) often leads to aerosol formation (see "Source B" in Figure 2.1). This expansion is a result of abrupt growth of vapor bubbles in the jet that break it up to attain a final two-phase equilibrium state. This phenomenon is commonly referred to as flashing. Unlike the shear flow phenomenon experienced in subcooled liquid jets, the mechanism of aerosol formation for superheated releases is dominated by flash atomization and the transition of thermodynamic processes to a vapor-liquid equilibrium. The relatively small-diameter particles produced in the flash atomization and the high volatility associated with a superheated release may lead to persistence of the aerosol component for an extended period of time. The reference pressure used in determining if a release is superheated is typically taken to be atmospheric pressure.

The flashed fraction (the fraction of the liquid release that immediately "flashes" to vapor) may be estimated by means of a heat balance across the outlet orifice where the decrease in latent heat of vaporization and increase in heat capacity are accounted for as the initial temperature approaches the critical temperature. As the discharge stream experiences a sudden pressure decrease following release from storage, some of the liquid will flash immediately to vapor, whereas the remaining liquid will either be suspended as a finely distributed aerosol or fall to the ground and form a pool. Generally, a superheated liquid jet or spray must be present for significant quantities of liquid droplets to be suspended in the initial cloud. In contrast to a subcooled liquid jet, a greater fraction of the aerosol component remains aloft and subsequently evaporates in flight, with the remainder falling onto the ground to form a boiling or evaporating pool. Elevated jets of liquefied ammonia, for example, have been observed to remain entirely airbornc (as both vapor and liquid droplets) unless the released jet impinges directly onto a surface (Herman 1987).

2 Examples of models for calculating aerosol droplet diameter include Iannelo et al. (1989), Tilton and Farley (1990), and Woodard and Papadourakis (1995a). 
A knowledge of the distribution of droplet sizes can be used to detemine the rainout fraction. Kitamura et al. (1986) and Bettis et al. (1987) have experimentally observed that the droplet sizes are log-normally distributed. As an example of droplet rainout, one criterion states that the liquid droplet will return to the ground substrate when the inclination of the droplet trajectory with respect to the vertical direction is greater than the half angle of jet expansion at the start of entrainment. The subsequent droplet settling velocity may be calculated by solving the balance equation expressing the equality between the force of gravity on the droplet and the upward-acting viscous and drag forces. Assuming that the droplet is a sphere in laminar flow, a critical drop diameter, $d_{c}$, can be calculated above which the droplets rain out.

The rainout criterion presented above is confined to the source release point and does not enter into the dispersion calculations. However, aerosols formed in an upwardly directed release, for instance, may exhibit a much Ionger airborne residence time and should be treated in a dispersion model that predicts the near-field transport of contaminant. Furthermore, volatile compounds in a vapor-aerosol system approach thermal and phase equilibrium much more slowly than pure vapor releases. This equilibrium time scale may depend upon relative humidity, initial gas-phase concentrations, aerosol mass concentration, aerosol size, temperature, accommodation coefficient, and chemical composition. During this transition period to equilibrium, complex heat transfer effects may be present in the cloud that subsequently affect the overall dispersion characteristics of the cloud. Additional discussions on incloud heat and mass transfer when aerosois are present in a dispersing cloud may be found in Banerjee et al. (1994) and Woodard et al. (1995b).

Liquids and vapors used or stored near their saturation temperatures in pressurized tanks may upon release to the ambient environment flash to a liquid-vapor mixture much in the same manner as superheated liquid releases. High-momentum, two-phase releases are typically caused by overpressure buildup and subsequent leakage or relief valve venting in runaway chemical process reactions.

Two-phase releases (liquid-vapor mixtures stored near their saturation temperature) typically represent one of the most complex source emissions that a vapor dispersion analyst will encounter. The liquid jet release phase is the metastable state to which a superheated release progresses to a two-phased equilibrium state between vapor and liquid phases for any release under saturation conditions. These cases may necessitate a treatment of the interphase heat, mass, and momentum transfer rates accompanying changes in cloud temperature or other conditions. Models that address such phenomena will generally peform a mass, momentum, and energy balance.

A general overview of the fundamentals of two-phase jet releases is given, for example, in Chapter 6 of Fthenakis (1993). A more detailed discussion of two-phase flow models can be found in the AIChE's Design Institute for Emergency Relief Systems (DIERS/AIChE 1992). A simplified method for characterizing flashing and non-flashing two-phase flow assuming no phase slippage and thermal equilibrium between both phases is given by Leung (1996). 


\subsubsection{Particulate and Vapor Dry Deposition and Resuspension}

Dry deposition due to impingement or sorption of a contaminant onto the ground surface may be influenced by aerodynamic roughness, atmospheric stability, contaminant concentration, relative humidity, turbulence, wind speed, and other meteorological factors. In the case of gaseous sources, the retention of material at the surface is entirely dependent on molecular penetration or cohesion.

The two most common methods for modeling vapor/particulate fallout mechanisms are the "partial reflection" and "source depletion" models. The partial reflection model generally works best in the near-field downwind range of the source and usually requires some modification of the underlying dispersion equations to account for reflection of a vapor plume back into the dispersing medium. The source depletion model relies heavily on the use of a "vapor deposition velocity," which is in turn a function of wind speed, friction velocity (i.e., a velocity scale established by the surface shear stress), wind stability, and surface roughness. Numerous deposition modeling approaches exist, varying from the simple methods used in D2PC (Whitacre et al. 1987), where the deposition velocity is multiplied by the spatial dose gradient to obtain vapor cloud deposition, to more sophisticated treatments where the settling velocity appears as a term in the boundary condition imposed on the stochastic turbulent transport equation. The turbulent transport of the contaminant along the vertical concentration gradient, in the later approach, is balanced by the net contaminant flux to the ground surface (Chrysikopoulos et al. 1992). The EPA's Industrial Source Complex (ISC 3) model (EPA 1995) uses a resistance method to calculate deposition velocity. This approach includes explicit parameterizations of the effects of Brownian motion, inertial impaction, and gravitational settling. The combined phoretic and stefan flow effects on aerosols near the surface is accounted for by a small adjustment to the deposition rate $(+0.01 \mathrm{~cm} / \mathrm{s})$.

Resuspension of solid particulates either from a solid or liquid spill are generally more of a hazard in the context of radionuclide releases. Two possible examples of when these types of releases are a concern in chemical dispersion are spillage of beryllium or lithium hydride powder. The most likely mechanism for transporting these chemicals into the atmosphere would be a fire where the fine particulates would be entrained into the high-temperature rising plume, deposited downwind onto the ground surface, and then subsequently resuspended into the atmosphere.

\subsubsection{Momentum- or Buoyancy-Dominated Plumes}

As a consequence of pressure or temperature conditions initially present during a release, a vapor emission may first form a momentum- or buoyancy-dominated plume. A vapor plume is first distinguished as a momentum-dominated plume or a buoyancy-dominated plume in order to select a model that emphasizes the prevailing flow and dispersion mechanism, namely momentum transfer in the first case and sudden changes in medium density in the second (see "Source C" in Figure 2.1). No universally accepted criterion currently exists for distinguishing between momentum- and buoyancy-dominated plumes. One of the simplest methods ${ }^{3}$ classifies a

3 List (1982), section on "Turbulent Buoyant Jets." 
vapor release as a momentum plume when the characteristic momentum length exceeds the characteristic buoyancy length.

The importance of quantifying this dichotomy between mornentum- and buoyancydominated plumes has been diminished in some of the more recent models that quantify jet dispersion based on solution of the conservation equations or direct simulation, in contrast to using empirical fits to trajectory data. Nonetheless, it still serves as a useful conceptual model for understanding puff and plume dispersion.

Momentum-dominated plumes are generally associated with highly pressurized releases. Momentum-dominated plumes typically experience rapid dispersion and loss of momentum and quickly decay to a state of positive, neutral, or negative buoyancy. Hanna et al. (1982) provide estimates of the time to transition as a function of the plume momentum flux and the initial buoyancy flux.

If a release does not satisfy the criteria for a momentum-dominated plume, but the temperature difference between the vapor contaminant and the surrounding air is sufficiently large, or if the molecular weight of the contaminant is less than that of air, a buoyancy-dominated plume will be formed. Many models use the "effective height point-source" model whereby the plume elevation at the transition point where the plume entrains sufficient quantities of air such that it achieves neutral buoyancy is used in the Gaussian dispersion equation. It has become customary to neglect vapor exposure to ground-level individuals upwind of this transition point. This procedure is based on the assumption that the compensating dispersion forces of upward buoyancy are adequate to ensure that the actual vapor concentration is negligible. Such an approach, however, may in some cases be underconservative. An example of a model in which ground-level exposure is associated with a rising plume through a succession of industrial point sources may be found in Netterville (1989).

\subsubsection{Dense Gas, Neutrally Buoyant, and Positively Buoyant Releases}

This discussion focuses on the physics of a quiescent (sometime referred to as passive ${ }^{4}$ ) release of vapor with density either exceeding, equal-to, or less-than that of the surounding ambient air (see "Source D" in Figure 2.1). Dense gas releases also play an important role in the subcooled and superheated/two-phase releases described in Sections 2.1.1 and 2.1.2. The treatment of dense gas releases under high-pressure conditions requires a generalization of the discussion below by considering specially adapted plume rise models (see Hanna et al. 1996).

4 The term "passive" is aiso commonly applied to neutrally buoyant releases in general. 
The differences in density between the released chemical and the ambient air induce four primary effects on vapor dispersion: 5

1. A self-generating velocity field induced by the horizontal density difference produces a plume or cloud with increased horizontal and reduced vertical extent in comparison with a similar release with no density difference.

2. The velocity shear introduced by a self-generating velocity field, described above, may lead to entrainment of ambient air, which enhances turbulent mixing and plume/cloud dilution.

3. The variation of density in the vertical direction will be stably stratified, and turbulence and mixing may be reduced or entirely inhibited in the central portion of the plume/cloud.

4. The inertia of the released material (more predominant in the case of elevated dense jets) is directly dependent upon the density of the chemical release.

Negatively buoyant emissions, commonly referred to as dense gas releases, undergo what is often referred to as gravitational slumping. Because of the inertial and momentum effects associated with a dense gas release, the initial vapor cloud displays a much stronger tendency to resist the hydraulic pressure field induced by the prevailing winds than in the case of a neutrally buoyant release that acts effectively as an ensemble of passive tracer particles. Subsequently, a dense gas release generally undergoes much more lateral (crosswind) spreading in comparison with a passive release and may even disperse upwind from the source location because of the hydrostatic forces induced by large density gradients. In the early stages of the dense gas dispersion, air entrainment may be relatively small in magnitude, and subsequent plume concentrations tend to decrease gradually with downwind distance. A more detailed discussion of the physical phenomena of dense gas dispersion is given in Britter and McQuaid (1988) and AIChE/CCPS (1996).

Many chemical compounds have a molecular weight exceeding that of air and, therefore, form dense gases purely by virtue of excess density. However, molecular weight is not the only factor that leads to dense gas behavior. Dense gas effects can also appear in releases that are colder than air and/or contain aerosols formed in the process of a rapid liquid release from a pressurized vessel. The canonical example of a chemical possessing a molecular weight less than that of air forming a dense gas release is anhydrous liquid ammonia $\left(\mathrm{NH}_{3}\right)$. The boiling temperature is approximately $-34 \mathrm{O} F$ at ambient pressure. As the vapor evolving from a

5 A more detailed discussion of the physical phenomenon of dense gas dispersion is given in Britter and MeQuaid (1988) and AIChE/CCPS (1996). 
boiling/evaporating liquid pool of $\mathrm{NH}_{3}$ mixes with the surrounding air, the mass of the ammoniaair mixture is sufficiently cooled that its density increases significantly above the surrounding ambient air, and a dense gas cloud is formed. Even in the presence of appreciable quantities of aerosol in the cloud, the density of the cloud, taken to be the weighted average of the flashed vapor and the unflashed liquid aerosol component when there is no phase slippage, will exceed that of air.

Several criteria exist for determining the conditions when a dense gas release has been sufficiently diluted that it no longer exhibits dense gas characteristics. However, no criteria can predict precisely where the transition from dense gas to passive gas behavior occurs. Some models use a critical Richardson number, ${ }^{6}$ which can range from 1 to 100 (typically 50 is used), to determine if dense or passive gas effects dominate. This number should not be thought of as an absolute value that separates dense-gas-dominated from passive-gas-dominated dispersion (AIChE/CCPS 1996). Therefore, models with dense gas algorithms typically employ a gradual transition between dense gas and passive gas behavior.

Although most chemicals handled in the DOE complex would, if released to the environment, initially form a dense vapor cloud, ultimately, with sufficient time and dilution, all releases transition to a neutrally buoyant cloud. Under these conditions, the vapor contaminant acts as a passive tracer responding exactly with the ambient air flow field. A number of criteria can be imposed on the transition between a dense or a positively buoyant (lighter-than-air) release (e.g., conservation of mass flux through the transition plane, preservation of isoconcentration surfaces and their continuity at the transition point, etc.). An important evaluation criterion when selecting a code for use in a dispersion analysis is to understand some element of how these transitions are accomplished and what inputs are required (e.g., does the user have to specify the concentration profile at the end of the previous transport regime?).

Positively buoyant releases usually appear in the form of buoyancy-dominated plumes, which are covered in Section 2.1.4. Converse to the case where a positively buoyant release transitions to a passive vapor cloud, there is an important class of releases where a vapor emission that is initially dense or passive actually transitions to a positively buoyant vapor cloud. This phenomenon is termed delayed lift-off, whereby the vapor cloud chemically reacts with the air (more precisely the water content of the air) and generates sufficient thermal energy through an exothermic reaction to effectively reduce the density of the cloud below that of the surounding air.

\subsubsection{Building Wake and Stack/Building Downwash}

Historically, nearly all hazardous vapor cloud models have ignored the influence of buildings on ground-level releases because of the belief of model developers that these obstacles tend to enhance urbulence and therefore decrease vapor concentration. Limited experimental data imply that for plumes with vertical (laterai) spread exceeding building height (width): (1) the

6 The Richardson number can be defined as the ratio of the initial potential energy of the vapor cloud to the ambient kinetic energy. 
concentration profile for ground-level vapor sources near buildings is strongly affected for approximately five building heights, ${ }^{7}$ and (2) plume width is increased, mixing is enhanced, and the mean concentration is decreased. However. under certain circumstances, vapor cloud releases near structures may result in increased concentration. A ground-level release that is constrained between two buildings is an example of a situation that can lead to this result.

Prediction of vapor dispersion in the presence of buildings and complex topography is a currently ongoing source of intensive research. The spectrum of computational methods for treating such types of dispersion analyses varies from hand calculation procedures captured in the correlations of Britter and McQuaid (1988) to passive or buoyant releases with the ISC3 model (Huber and Snyder 1982; Huber 1977; Scire and Schulman 1981) to three-dimensional NavierStokes equations solvers such as FEM3C, which is evaluated in this study.

Vapor emissions from short stacks and vents located on top of buildings may also be subjected to a phenomenon called downwash. In downwash conditions, vapor concentration is increased above levels normally expected for downwind receptors below the source elevation because the effective source elevation is reduced by means of entrainment of the vapor plume into the wake or circulation cavity of the building or stack. The vapor emission rate, source area dimensions, building dimensions, and building configuration infiuence the cavity and wake effects from the structure. The length of the building cavity is typically between two and five times the building height.

\subsection{METEOROLOGICAL INFLUENCES}

Meteorology that is representative of the conditions that could occur at the site in question or at the time of the actual release should be used to assess the consequences of the most probable or likely potential or past accidental release. Although meteorological measurements taken at the nearest National Weather Service (NWS) surface and upper air station are generally acceptable, some modeling applications may require that appropriate values of wind speed and direction, atmospheric stability, surface roughness, temperature, and relative humidity should be obtained from on-site meteorological measurements. Guidance on what applications would warrant the use of on-site measurements is provided in Section 6.2.2.1. Specific guidance on the collection of on-site meteorological data is given in the U.S. Environmental Protection Agency's (EPA's) On-Site Meteorological Program Guidance for Regulatory Modeling Applications (EPA 1993a) and in ANSU/ANS-25 (1984). Some of the key meteorological variables that influence the consequences of a vapor cloud release are highlighted below. Ground-surface effects are discussed here because of their direct influence on the meteorological parameters that affect a vapor cloud's dispersion and transpor. More in-depth discussions on vapor cloud and air

7 Generally the flow fields in the wake of buildings can be characterized by turbulence levels of two zones - the cavity and wake regions. The cavity region is distinguished by intense, rapidly varying turbulence or "chaotic flow." This region extends downwind to about threc building heights (below streamline separating displaced flow and trapped or entrained flow). The wake region is distinguished by extreme (near wake to the near upwind flow) turbulence levels. This region is assumed to extend from about 3-10 building heights downwind for passive or buoyant releases. 
poliution meteorology, including source physics and thermodynamics, can be found in Pasquill (1974), Randerson et al. (1984), and AIChE/CCPS (1996). More general references include Hanna et al. (1982). Turner (1994), and the American Meteorological Society's Handbook on Meteorology (Houghton 1985).

\subsubsection{Wind Speed}

Wind speed affects the concentration within a vapor cloud released to the atmosphere in many ways, such as by influencing plume dilution, the rate of evaporation or mass transfer from a pool, and plume rise from a buoyant release.

Downwind concentrations from neutrally buoyant ground-level releases generally tend to vary in inverse proportion to wind speed. As a result, non-dense-gas models account for dilution by predicting proporionately decreasing concentrations with wind speed increases. In contrast, wind speed dilution effects on concentrations initially tend to be less pronounced for dense gas releases. In light winds, dense clouds tend to be "pancake-shaped" near the source and may remain rather flat and at maximum concentrations until farther downwind. With increasing wind speed, the region of maximum concentration moves away from the source, and the downwind hazard zone tends to increase with elongation of the vapor cloud. As the rate of air entrainment is increased at higher wind speeds, the hazard extent of dense clouds decreases. In addition to accounting for these dilution phenomena, most dense gas models predict a dramatic reduction in lateral spread of a ground-released vapor cloud with increased wind speed.

Meteorological conditions also play a crucial role in liquid vaporization. The rate of evaporation from a liquid pool and resulting downwind concentration increases with wind speed and with incoming solar radiation. Because of the inverse dilution effect of wind speed, concentration increases due to wind speed will be moderated and under certain conditions (e.g., low-volatility liquids) may be canceled or slightly reduced. Heat transfer between the pool and the underlying surface also affects the evaporation rate. Surface roughness and surface substrate composition (e.g., concrete, soil, clay) have an important influence on the heat transfer rate. The APAC Spills report (Brereton et al. 1995) provides a more detailed discussion of the relationship between pool boiling or evaporation and ambient conditions and meteorology.

The vapor rise due to buoyancy of a themally dominated cloud or upward-directed jet is reduced with increasing wind speed. Therefore, as in the case of evaporating pools, ground-level downwind concentrations from rising plumes and upward-directed jets tend to increase with increasing wind speed. However, there is a limiting feature to this phenomenon since the dilution effects of increased wind speed will moderate the concentration rise. Under low wind speeds, initial plume rise (due to jet momentum and/or the heat buoyancy effects) can be large, and downwind concentrations can be small. At higher wind speeds, the air's horizontal momentum reduces the jet's vertical momentum and the vapor cloud's thermal buoyancy, resulting in a bentover jet or cloud. A dense cloud released by a vertical jet may bend over and sink to the ground with moderate wind speeds. However, the density of the cloud, its release height, and the level of atmospheric turbuience can moderate or intensify the degree of bend and how fast the cloud reaches the surface. 


\subsubsection{Ambient Temperature, Relative Humidity, and Atmospheric Pressure}

Many chemicals are hygroscopic and react with the moisture content of air. These chemical transformations may dramatically change the physical-chemical behavior of a vapor release and subsequently alter its dispersion characteristics. In the case of hydrogen fluoride (HF), for instance, its hygroscopic behavior in the atmosphere transforms it from an compound with essentially neutral buoyancy to a dense gas vapor cloud.

The initial density of a pressurized liquid jet release forming a liquid-vapor mixture, liquid aerosol, or droplets with "flashed" vapor generated from the release thermodynamics can be estimated by first computing the flash fraction. The flash fraction can be estimated with a simple adiabatic flash calculation or through a number of more sophisticated methods (Fauske and Epstein 1988; Leung and Grolmes 1988; Leung 1992), including the techniques mentioned in Fthenakis (1993) for two-phased flow. Two-phase and superheated liquid jets are typical of this type of release. As the cloud moves away from the release point, changes in cloud density depend on the rate of heat transfer into the cloud. This heat transfer is a function of temperature differences between the vapor cloud and the ambient air, the relative humidity, and the atmospheric pressure. As an example, the effect on a vapor cloud formed from a subcooled jet release at ambient temperatures above the vapor temperature is to heat the cloud and therefore reduce its density. This reduction in density enhances the cloud buoyancy and narrows the initial cloud dimension. On the other hand, the effect of heat transfer on a vapor cloud formed from a superheated jet release at ambient temperatures above the vapor temperature is to partially vaporize suspended cloud droplets and reduce the cloud density. This effect is mitigated or reversed by the vaporization process in the cloud, which tends to increase its density by reducing the cloud temperature below its boiling point. Predicting aerosol cloud dynamics and behavior is further complicated by the effects of vapor density change from potential chemical reactions within the cloud (e.g., hydrogen fluoride polymerization) and/or the influence of ambient humidity from moist air entrainment (e.g., pressurized anhydrous ammonia release).

\subsubsection{Atmospheric Turbulence and Diffusion}

Turbulence in the atmosphere consists of a continuous spectrum of eddy scales, as opposed to a discrete set of scales. The concept of turbulent eddies has been commonly used to help visualize and understand the complex nature of turbulent flow. A turbulent eddy can be thought of as rotating or swirling motion with a characteristic dimension defined at a local scale. Vigorous stretching of eddy vortices is required to maintain the ever-present fluctuating vorticity inherent in a turbulent atmosphere. In the atmosphere, eddies overlap, with larger ones carrying smaller ones. This overlap features a cascading process, whereby as turbulence decays, kinetic energy transfers from larger eddies to smaller ones (Wilcox 1994). Uitimately the smaller eddies dissipate to heat through the action of molecular viscosity. Therefore, turbulent flows are always dissipative. Turbulence is characterized by random fluctuations that obviate a deterministic approach to the problem. Although most of the commonly applied diffusion models still apply deterministic techniques, stochastic approaches that treat the atmosphere as a "chaotic" or fluctuating system are receiving increased attention. 
All of the models reviewed in this report have theoretical-empirical algorithms for estimating lateral and vertical spread or diffusivity (i.e., $\sigma_{y} / \sigma_{z}, K_{y} / K_{z}$, or $\mathrm{K}-\varepsilon$ ) of a plume or puff with distance or time from the release point. These algorithms require the user to provide an estimate of the energy state or degree of turbulence in the atmosphere, which can be characterized by a continuous spectrum of energy states employed in diffusion models with a stochastic approach, or, more commonly, as discrete categories referred to as stability classes. Models using discrete turbulence methods must identify stability class to estimate $\sigma_{y}$ or $\sigma_{z}$. Without research-grade turbulence measurements, the stability class must first be determined, preferably by a simple typing scheme or method based on routinely available or easy and inexpensive measurements. Stability classification methods can generally be grouped into one of three broad approaches: solar radiation, wind fluctuation, and similarity criteria. When the dispersion parameters are combined with data obtained from tracer experiments, the degree of vertical and horizontal pollutant spread can be estimated as a function of downwind distance and stability class.

The EPA recommends and ranks four alternative schemes for estimating the PasquillGifford (P-G) stability category from on-site meteorological measurements. The agency has traditionally favored an approach for estimating the P-G stability category using a solar radiation criterion method proposed by Turner over 20 years ago. The "Tumer method" requires the use of site-specific wind speed measured at or near $10 \mathrm{~m}$ and representative cloud cover and ceiling height. The method is based on the foundation originally proposed by Pasquill (1961) and Gifford (1960) and modified slightly by Tumer (1967). Pasquill (1961) introduced a method of estimating the atmospheric stability, incorporating considerations of both mechanical and buoyant turbulence. ${ }^{8}$ He presented information on the lateral and vertical spreading of diffusing plumes as a function of six atmospheric stability classes designated A to $F$. Atmospheric conditions are described as strongly (A), moderately (B), and slightly (C) unstable; neutral (D); and slightly (E) to moderately (F) stable. ${ }^{9}$ The mechanical turbulence is considered by the inclusion of the surface wind speed (measured at approximately $10 \mathrm{~m}$ above ground). The positive generation of buoyant turbulence is considered through the insolation (incoming solar radiation). The negative generation of buoyant turbulence is considered through the nighttime cloud cover. The less the cloud cover the greater the amount of heat that escapes from the surface through infrared radiation. High wind speeds or overcast cloudiness will produce neutral conditions, D class stability. These stability categories are based on five classes of surface wind speeds, three classes of daytime insolation, and two classes of nighttime cloudiness and ceiling.

It is recognized that the "Tumer method" incorporates subjectively determined insolation assessments based on hourly cloud cover observations. A second solar radiation criterion method, solar radiation delta-T (SRDT), has in recent years been gaining favor as the

8 Frank Pasquill's dispersion coefficient and stability classification scherne forms the basis of EPA's regulatory models. His imponant contributions to the field of air pollution meteorology are the subject of a special 15-papcr memorial issue of the Joumal of Applied Meteorology (August 1997).

9 Turner (1964) incroduced a seventh stability class, sometimes referted to as G. for strongly stable conditions and low wind speeds. Because of the rarity of occurrence of conditions leading to $G$ stability, this class has not been incorporated in most models using the PG/T system. 
preferred stability classification alternative. Although EPA's Guideline for Air Quality Modeling (EPA 1996b) does not recommend the SRDT method over the Turner method, it does recommend that in the absence of requisite data to impiement the Tumer method, the SRDT method may be used. The SRDT method obviates the need for subjective cloud cover and ceiling observations (non-instrumented or human). The SRDT approach, like Turner's method, uses surface wind speed (at or near $10 \mathrm{~m}$ ). However, instead of using cloud cover and ceiling observations, it use measurements of total solar radiation during the day and low-level vertical temperature difference $(\Delta T)$ at night. The SRDT method was originally proposed by Bowen et al. (1983) and modified by EPA (1987) to retain as much as possible the structure of the P-G/T method implemented in EPA's meteorological preprocessors (e.g., MPRM and PCRAMET). The details describing and supporting the SRDT method are given in documents issued by EPA (1993a, 1997). EPA-endorsed alternatives to the use of solar radiation criterion approaches include two methods based upon wind fluctuation criterion (sigma phi and sigma theta). These methods are described in the EPA guidance $(1987,1996 \mathrm{~b})$. Although not mentioned in that guidance, some models employ the capability to use a stability scheme based on similarity criterion. Examples include approaches, originally developed by Golder (1972), that use the Monin-Obukhov scale length.

Further details and discussion are given in Hanna et al. (1982), AIChE/CCPS (1996), and EPA (1993a) on solar radiation, wind fluctuation, and the similarity criteria approach to stability classification and associated dispersion parameterization used in various dispersion models reviewed in this report. Comparisons of the differences in various methods of stability classification can be found in EPA (1993a), Sedefian and Bennett (1980), Weber and McDonald (1977), Kretzschmar and Mertens (1980), and Mitchell (1982). A fuller account of the basics of atmospheric turbulence in density stratified flows can be found in Panofsky and Dutton (1984) and J.S. Turner (1973).

\subsubsection{Ground-Surface Effects}

The heterogeneity or complexity of the underlying surface has direct influence on winds, temperature, pressure, relative humidity, and turbulence. This influence can have a pronounced effect on the diffusion and ultimate behavior of a vapor cloud or plume. Mechanically and thermodynamically induced ground-surface effects can occur in two types of "complex terrain" - rugged or irregular terrain such as steep hills, valleys, or mountains, and land-water terrain such as lake-land or sea-land interfaces. Rugged terrain does not include "gently rolling" land surfaces, and land-water terrain does not include land interfaces with relatively small water bodies (e.g., a tributary or narrow river). Both thermal (i.e., anabatic and katabatic winds in mountain valley settings) and mechanical forcing are important influences on flow patterns in irregular terrain. Thermodynamic influences tend to dominate land-water terraininduced flow pattems. However, if the land interface is irregular (e.g., abrupt up- or down-slope) or is urban, both mechanical and thermal effects can have pronounced and very complicated influences on vapor cloud transport and ultimate fate.

The boundary-layer phenomena induced by flow over irregular terrain include, but are not limited to, flow separation formed from channel and gap winds, density stratification with height, diurnal thermally driven slope or drainage winds, vortices shed from terrain obstacles, 
and wind-shear-generated turbulence enhancement. Although much uncertainty exists about the effects on downwind or down-slope vapor concentrations from streaming, vortex capture, and flow channeling, physical reasoning implies that these complex flows may constrain vapor dispersion and in some cases even form a contaminant "attractor." Further discussion of these complex phenomena and other rugged terrain phenomena can be found in Eagan (1975), Hovind et al. (1979), and Dickerson et al. (1980).

Putting these complexities aside, some general observations can be made about slope flows of dense vapors for which the scale of the release is small compared with the topographic feature or slope (Britter and McQuaid 1988). The plume/cloud widens and dilution is enhanced for an up-slope wind, and the plume/cloud and dilution is decreased for down-slope winds. Dense vapor cloud motions that are influenced by slopes are retarded by surface stress and ambient air entrainment, with dominance by the latter for slopes greater than about one degree. Entrainment will be enhanced by up-slope winds and retarded by down-slope winds, because of influence of velocity shear.

The boundary-layer phenomena induced by flow over terrain with a land-water interface include, but are not limited to, three-dimensional helical gradient onshore flow due to differential heating between land and water surfaces and lake/sea breeze mesoscale circulation. The influence of this phenomenon has primarily been studied and applied to elevated releases of positively buoyant plumes near shorelines. Although mention is made below of the effects of gradient onshore flow on entrainment and dilution of dense vapor clouds (e.g., vertical jets), the relative importance or significance of these effects is not well known.

Gradient onshore flow occurs when differential heating between colder water surfaces and warmer land surfaces (e.g., typically in the spring in the Great Lakes) establishes a horizontal pressure gradient that provides the driving force for the inflow across the shoreline. Once established, a thermal internal boundary layer (TIBL) is formed primarily due to the combined effect of the change in surface roughness in the transition from flow over water to flow over land and the rapid warming of the colder air coming from flow over the water surface by the relatively warmer air over land. This phenomenon separates the heated air from below from a cool stabie layer above. The degree of heat, momentum, and moisture exchange between the two air masses governs the shape and rate of the TIBL formation. Surface or low-level vapor clouds released near the shoreline are initially dispersed in an unstable atmosphere with a very low TIBL ceiling near the shoreline. The low TIBL ceiling height would limit vertical dispersion of vapor clouds released near the shoreline. The effect of gradient onshore flow on air entrainment and dilution of dense vapor clouds depends on the strength of the advection and vapor cloud to air temperature differential. Vertical vapor jet or buoyant releases near the shoreline are advected inland above the TIBL initially until they reach the TIBL boundary. At this intersection, the vapor cloud is rapidly fumigated into the turbulent layer below (i.e., sea breeze formation), which can lead to high contaminant surface concentrations. A continuous plume release can be fumigated for up to 8 to 10 hours.

Conditions leading to the development of lake/sea breeze circulation include (1) very light-gradient winds, usually associated with an offshore high-pressure system, (2) strong 
insolation, which is usually less than 60\% middle to high cloud cover (non-convective cloudiness), and (3) inland daytime temperatures rising above lake or sea surface temperatures. Under these conditions a lake/sea breeze will form, which can be confirmed by (1) a shift of winds from offshore to onshore during the day, and (2) the presence of a definite front or convergence zone separating surface air streams with over-water trajectories. The convergence zone is typically referred to as the lake- or sea-breeze front or wind shift line. The lake/sea breeze will not initiate with extremely light onshore flow or with a geostrophic flow that is parallel to the shoreline. Once established, mesoscale circulation fonmed in a lake/sea breeze can recirculate and accurnulate vapor cloud contaminants.

More in-depth discussions of modeling the physics and air pollution meteorology associated with land-water interaction can be found in Cagnetti et al. (1988), Luhar et al. (1995), Hanna et al. (1985), DiCristofaro et al. (1992), Venkatram (1986), Lyons (1975), Raynor et al. (1979), and Pielke (1981).

\subsection{ROLE OF HAND CALCULATIONS}

It would be a restatement of the obvious to say that hand calculations, wherever applicable, practical, and sufficiently accurate, are acceptable, if not preferred, in lieu of computer calculations. Because of their "transparency," hand calculations generally lend themselves to easier verification and often make it possible for the analyst to detect the parameters or variables to which the model is most sensitive by simple inspection or straightforward analysis.

Unfortunately, most realistic dispersion scenarios are sufficiently complicated that they preclude the use of simple hand calculations without first applying (in some cases grossly) conservative assumptions. Therefore, in most cases the price for simplicity is conservative results. Nonetheless, hand calculations are of a great value to the analyst when conservatism is justified - namely in the case of scoping calculations (Category 1) or hazard screening (preliminary hazard analysis) as two common examples. Hand calculations afford an easy and relatively quick means of assessing whether a particular release scenario exceeds the established site impact criteria and thereby merits more detailed examination or represents a sufficiently low hazard that it may be removed from further consideration.

This section concludes with a brief summary and sampling of some references pointing to hand calculational methods that can be used for various classes of dispersion phenomena.

\subsubsection{Source Geometry Approximations}

Hazardous chemicals can be released to the atmosphere either from a point source or through a distributed line, area, or volume source. Most hand calculational methods using the Gaussian plume/puff approach assume a point-source release. In general, it is difficult to approximate any source configuration other than a point or line in conjunction with the Gaussian dispersion model due to either gross errors in approximation or the heavy computational penalty. 
Applying the point-source dispersion model to a distributed area release normally overestimates downwind concentrations, particularly for receptors near the release location. To alleviate this problem, dispersion coefficients in the Gaussian equation may be estimated by accounting for the initial size of plume/puff when a point-source dispersion code is used for a distributed area source. Commonly used approaches include (1) the use of the initial dimensions of the puff or pool, and (2) the use of initial peak concentration in the plume/puff.

For the first approach, initial dimensions of the puff or pool are used to estimate initial lateral and vertical plume spread. In the case of the second approach, a virtual transition parameter is introduced into the $\sigma_{y}$ and $\sigma_{z}$ parameters to force the Gaussian continuous plume and puff equations to agree with a prescribed concentration value at the source location. Finally, this approach can be even further extended to ensure that a continuous, mass-consistent vapor concentration profile is preserved for receptor locations falling within the transition region between continuous plume and instantaneous puff (Hesse 1991).

Simple dense gas models provide hand calculational methods for estimating the gravitational slumping and vapor dispersion in calm air conditions using a simple slab or box model (see the van Ulden model described below in Section 2.3.6). There is a certain level of arbitrariness or indeterminacy in many of these approaches since either the initial radius or height of the vapor cloud must be specified - data that are difficult to formulate under most release conditions.

\subsubsection{Jet Dispersion}

Assuming that a pure vapor jet is released into relatively calm air (wind speed not exceeding $1 \mathrm{~m} / \mathrm{s}$ ) and that the downstream distance is sufficiently large that the jet density is approximately equal to that of the surrounding air, Mills and Paine (1990) describe a hand calculational procedure for obtaining the vapor concentration as a function of downwind distance, as well as the arrival time of the leading edge of the jet to the receptor location. Other more general methodologies for calculating jet dispersion that lend themselves to multistep handcalculational methods include Hwang and Chiang (1986) for buoyant jets in crossflow and TNO (1992) for turbulent free jets.

\subsubsection{Dense Gas Jet/Plume Dispersion}

In the instance of all vapor/gas dense plumes where internal turbulence dominates over ambient turbulence, a near-field model for maximum initial rise, downwind distance to plume touchdown, and the associated vapor concentrations is presented by Hoot et al. (1973). The analytic expressions for the variables described above were obtained by solving the equations of entrainment, conservation of horizontal and vertical momentum, and conservation of mass by assuming that the ambient cross-wind speed is constant, all gas-specific heats are equal, and the vapor distribution within the plume is "top hat" at any given distance from the source. 


\subsubsection{Positively Buoyant Plume Rise and Dispersion}

The plume rise equations derived by Briggs (1975) and adopted by the EPA are one of the most common methods for calculating the trajectory and centerline dispersion of a rising vapor plume and easily lend themseives to hand calculation. However, these equations form only one of an ever-expanding collection of analytic plume rise models.

\subsubsection{Stack and Building Downwash}

The physical phenomenon typically called "downwash," whereby the effective height of a plume may be suddenly reduced immediately downwind of the source, is described in detail in Section 3.2. One of the most recent collections of equations for calculating the critical wind speed and downwind distance to ground-level concentration from an elevated vapor plume release is presented by Bowman (1996). Bowman also describes the Schulman and Scire 10 (1980) and the Huber and Snyder ${ }^{11}$ (1982) treatments for downwash. All these methods lend themselves to simple hand calculation.

\subsubsection{Dense Gas Ground-Level Dispersion}

Straightforward correlations for instantaneous and continuous dense gas releases based upon small- and large-scale test data are presented by Britter and McQuaid (1988). The most important model assumptions are as follows:

- The Boussinesq approximation must apply to the release of interest (see Model Evaluation section).

- The Britter and McQuaid model (1988) represents a long-term averaged estimate of the downwind distance to a particular concentration.

- The ratio of the concentration of concern to the initial vapor concentration (same units) must be in the range of 0.002 to 0.1 .

- The density of the vapor release must equal or exceed that of the ambient air.

Britter and McQuaid (1988) also provide quantitative guidance on the effects of buildings on vapor concentration.

Another simple model, more commonly applied to "instantaneous" releases and based on approximate first principles, is the slumping cylinder model of van Ulden (1974), where radial

10 Downwind effects are accounted for through building dimension adjustments to the dispersion coefficients and incorporation of a line source in the plume rise equations.

11 Adjustments to the dispersion coefficients are made to account for buildings, but the effects on plume rise are not treated. 
spread is parameterized. and the entrainment of air through the sides of the cylinder is neglected in comparison to entrainment through the top of the cloud.

\subsubsection{Neutrally Buoyant Dispersion}

The Gaussian plume and puff equations for passive dispersion are thoroughly discussed in the handbook by Hanna et al. (1982). That handbook also describes some of the "rules of thumb" developed by Wilson (1979) for evaluating the effects of structures on vapor concentration.

\subsection{CONSIDERATIONS FOR RELEASE DURATION AND AVERAGING, SAMPLING, EXPOSURE, AND TRAVEL TIMES}

The consequences of an airborne toxic chemical release and its associated hazard impact zone may be measured in a number of ways. One means of defining the zone of concern is the maximum downwind distance where the instantaneous concentration exceeds an established minimum threshold value. However, since dispersion is a stochastic phenomenon exhibiting in some cases large variability in concentration values from one instant to the next, there must implicitly or explicitly be attached to each established concentration level the corresponding averaging time. Therefore, if hazard assessment is to be based on a concentration value to which exposure at concentrations equal-to or exceeding this value event for short moments is unacceptable, then relatively small averaging times should be employed in the dispersion analysis. On the other hand, many of the regulatory-based models use hour-to-hour variations in concentration as a means of estimating areas of impact. Thus, proper choice of averaging, sampling, and exposure times and their relation to source duration, travel time to the receptor of interest, and other factors are important considerations in any dispersion analysis.

When hazardous chemicals are released into the atmosphere, they are transported and dispersed by the atmospheric wind fleld, which is characterized by a great deal of random variability known as turbulence. The turbulent wind fluctuations cover a broad spectrum or range of time and space scales. As a result, as the time of dispersion of a plume of hazardous chemicals increases, the plume is being dispersed by a larger and larger range of wind fluctuations. Consequently, the width of the plume increases and its average centerline concentration decreases. Wilson (1995) and AIChE/CCPS (1996) provide comprehensive discussions on this topic. It is important to account for these various averaging times within dispersion models, which generally ask the user to input information concerning several types of times, as described below. However, the user should realize that not all models ask for this information, and different models ask for different combinations of subsets. The users guides should be carefully read in order to understand exactly what input parameters are required.

\subsubsection{Release Duration $\left(\mathrm{T}_{d}\right)$}

If there is a chemical accident, it will always have a [knite release duration, $\mathrm{T}_{d}$, which is defined as the time period when mass is being released to the atmosphere from the source. Eventually, the tank or pipeline will empty or someone will shut off a valve and the release will 
stop. It is possible that some releases may be nearly instantaneous (e.g., an explosion of a pressurized tank). Most common release scenarios are treated as if they are continuous and nearly constant for a finite time period. $T_{d}$, over which the chemical is escaping from the tank or pipe. Of course, in reality the release rate may be highly time variable, but most models cannot account for this variability, and those models that claim to account for the variability do so in an arbitrary and empirical way.

\subsubsection{Averaging and Sampling Time $\left(\mathrm{T}_{s}\right)$}

At any given sampling location, an instrument would observe a time series of concentrations that fluctuate because of atmospheric turbulence. Suppose concentration observations are taken every second. The magnitude of the fluctuations in concentration is typically the same magnitude as the mean or average value. Since the dose or toxic load (see Section 2.4 .3 below) is typically defined using an average concentration over some time period, it is necessary to average the concentration observations over an averaging time, $\mathrm{T}_{s}$, which refers to the total duration of time of the incident or the total time that a monitoring instrument is turned on.

\subsubsection{Exposure Time $\left(\mathrm{T}_{e}\right)$ - Dose/Toxic Load or Concentration Effects}

Toxicologists and epidemiologists have studied the health effects of many chemicals on humans and on laboratory animals. From these studies, they have derived estimates of concentrations that will cause certain health effects in humans over certain time periods. Compounds for which acute exposure data are available can be generally grouped as dosedependent or concentration-dependent chemicals.

Effects from dose-dependent chemicals are functions of both the concentration and exposure time or duration. The time periods of acute exposure interest range from a few seconds up to about an hour. However, because the data only cover a small part of the range of interest, it is often necessary to extrapolate the data by using concepts such as toxic load or dose. For example, suppose the data were all taken over times of one hour, but the user is interested in a time of five minutes. Toxic load is defined as the integral over some total time, $T$, of the product of $C^{n}$ and time dt, where $C$ is the concentration, which is raised to some power $n$, and $d t$ is sone small time increment. If the power $n$ is unity, then the resulting integration produces the quantity known as the dose. For some chemicals such as chlorine, $n$ is greater than unity, since the same "dose" will have a worse effect when applied over a shorter total time period. Tables containing information on toxic effects and values of the power $n$ are available for some chemicals, but there is still much work to be done to complete the tables for all chemicals of interest to the DOE safety analyst. See, for example, the CCPS Guidelines for Chemical Process Quantitative Risk Analysis (AIChE/CCPS 1989) for a list of probit equation coefficients and exponents for 20 commonly used chemicals.

Concentration-dependent chemicals are defined as fast-action; their effects can be assumed to be immediate and correspond more closely to concentration than to dose. Included in this category are sensory irritants, such as anhydrous ammonia, and chemicals that are vesicant or 
corrosive in their action. such as phosgene. It should be noted that the fact that a chemical exhibits concentration-dependent effects does not preclude dose-dependent effects at much lower levels (Craig et al. 1995). Any chemical that has been assigned an emergency response planning guideline (ERPG) level, for example, must be considered to have concentration-dependent toxic effects. The American Industrial Hygiene Association (ADHA) Emergency Response Planning (ERP) Committee, which has developed and issued three levels of ERPG values (based on toxic effect) for 74 chemicals or chemical compounds, has advised not attempting to extrapolate to higher guideline concentration levels for longer exposure times (AIHA 1996). This caution is based on the fact that Haber's law $\left(C \times T_{e}=\right.$ constant) is not valid for any concentrationdependent chemical, nor for all dose-dependent chemicals. Specifically, the ERP Committee states: "Extrapolation to higher guidance levels for shorter exposure periods should not be attempted by use of the Haber relationship or modifications thereof without specific validating data." Use of the ERPG values is valid for exposure periods of less than one hour, but not for longer periods.

The ERPG values are listed in Appendix D, along with temporary emergency exposure limits (TEELs) developed by Craig $(1995,1996)$ for chemicals without an assigned ERPG level. The authors note that the ERPGs and the TEELs (unless otherwise stated) should be treated as ceiling or peak values; doing so would make the estimated concentration of interest an instantaneous value. For practical purposes, the peak 15 -minute predicted concentration may be treated as the instantaneous concentration for comparison with the ERPG or equivalent toxic value.

\subsubsection{Advection Travel Time $\left(\mathrm{T}_{t}\right)$}

The geographic position of the point of the chemical release is known, as well as the position of one or more downwind receptor points of interest (e.g., plant fence line, school, hospital). Given the distance, $x$, to one of these receptor points, plus a knowledge of the wind speed, $u$ (which is carrying the plume downwind), then the travel time, $T_{t}=x / u$, can be defined. This time is used to decide whether the plume should be treated as a continuous plume or instantaneous cloud for simulating dispersion.

\subsubsection{Instantaneous Versus Continuous Release}

Assume that a chemical is released over a finite duration of time, $T_{d}$, and that the cloud travel time from the source to the receptor is $T_{1}$. Then if the release time, $\mathrm{T}_{d}$, is much less than the travel time, $T_{1}$, and the resulting cloud is viewed from above (i.e., as if a helicopter was looking down and videotaping the cloud), the cloud will detach itself from the source before its leading edge reaches the receptor, and the cloud "looks" like a puff or an instantaneous release. Conversely, if the release time, $T_{d}$, is much greater than the travel time, $T_{1}$, then the cloud "looks" like a continuous plume. Britter and McQuaid (1988) suggest that the following ratios of release 
duration and travel time be used as a criterion to decide whether to model the cloud as an instantaneous puff or a continuous plume:

If $T / T_{i}<0.6$, release treated as instantaneous.

If $0.6 \leq T_{d} / T_{t} \leq 2.5$, take minimum value of concentration estimate at $\mathrm{x}$ from calculations using both a continuous and instantaneous model.

If $T / T_{r}>2.5$, release treated as continuous.

A $T d T_{\text {t }}$ ratio of less than or greater than unity has typically been included in many hazardous gas codes, which automatically make this decision as the calculations are proceeding.

\subsubsection{Variability in Approaches}

It is important to mention again that a variety of approaches are used in the many hazardous gas models to treat the effects of the averaging time, release times, and other parameters defined above. Some models do not account for these effects at all, and sometimes two models account for the same effect in notably different ways. A postprocessor may be needed to calculate toxic loads or doses. The model users guides should be carefully read regarding these matters. 


\section{MODEL SELECTION}

\subsection{CHEMICAL ACCIDENT ASSESSMENT PROBLEMS FACED BY SAFETY ANALYSTS}

Safety analysts and emergency management personnel were surveyed to identify accidents analyzed and models applied in support of SARs developed at DOE facilities. That survey provided insights into the chemical accident issues commonly faced by the safety analyst. Those issues are summarized as follows:

1. Specific guidance is lacking for addressing a wide spectrum of accident sequences.

2. Numerous models of varying pedigree and robustness are available.

3. Only a limited number of models are available to address complex airborne chemical behavior or dispersion physics (e.g., transformations, dense gases).

4. Analysis practices for chemical accident hazards are not as mature as those employed for radiological hazards.

5. No "best practices" have been identified relative to application for a full spectrum of specific problems.

In the absence of specific guidance in DOE Order $5481.1 \mathrm{~B}$, technical guidance, or other documentation, the safety analyst is limited in his ability to focus on the correct approach for performing the particular accident analysis that is needed. The analyst has to assume the burden of matching available methodologies to a wide spectrum of specific accident sequences, with no assurance that the technique chosen will accurately represent a real event. Therefore, the best case is that confidence in the result is somewhat limited; the worst case is that the analytical results are not representative of the actual risks to health and safety.

To further complicate matters, the analyst has access to a wide range of analytical tools in an essentially guidance-free environment. More than 100 atmospheric dispersion models are available in the public domain, each with varying degrees of comprehensiveness and robustness. Each of these models contains various levels of detail in the physics employed to treat atmospheric transport and dispersion. In addition, many have not yet been subjected to independent model performance evaluation necessary to ensure that they achieve the level of accuracy required to provide confidence in their results. It is not obvious to the safety analyst which model or technique would best fit the analytical needs of the accident sequence being considered.

Although numerous dispersion models are available, few of them adequately treat the complex source-atmosphere thermodynamics, physics, and chemistry of two-phased single and multicomponent releases. multicomponent pool evaporation, and atmospheric transformations. 
Most models fail to consider the thermodynamic effects of chemical mixtures on cloud formation and dispersion.

Mathematical creatment of the chemical accident sequence and subsequent chemical species redistribution by the atmosphere is technically more complex than is the corresponding radiological analysis. This difference is due mainiy to the fact that the chemical release process can involve situations that include complex chemical and thermodynamic interactions. An example is a release of pressurized liquid carbon dioxide, which cannot exist as a liquid at ambient atmospheric pressures. Part of the liquid carbon dioxide flashes to a gaseous form, while the remainder immediately becomes a "puddle" of snow. The snow then sublimates to carbon dioxide gas. This two-phased flow is common thermodynamic behavior for cryogenic or refrigerated liquids that are gases at normal atmospheric pressures and temperatures. Thus, to be effective, chemical dispersion models must be able to address the complex thermodynamics that occur at the release-atmosphere interface.

Guidance as to which codes are best to use for which types of chemical releases is generally absent. Thus, with a full spectrim of chemical accident sequences to be considered (e.g., two-phased flows, jets, superheated and subcooled liquids) and with a large selection of dispersion models available for use, the safety analyst is faced with a difficult task of relying primarily on professional judgment and experience to select the right model for the type of accident that is being evaluated. This circumstance can only be remedied by the development of "best practices;" that is, developing documentation that matches various problem types to the available models and approaches that best address those problems.

Development of best practices is one of the primary missions of the CDCA Working Group. To ensure that best practices can be developed for all problem types, a wide spectrum of chemical dispersion models needed to be evaluated. Initially, 135 models were identified, but these were carefully screened down to the more manageable number of 24 models. Those 24 models possess varying degrees of capability and applicability, but as a whole provide a suite of codes that the safety analyst can use to evaluate most chemical consequences that are possible at his facility.

\subsection{CODE SELECTION PROCESS}

The code selection process was initiated early in the project by first examiuing previous surveys of code availability. The most comprehensive list was found in a document titled Atmospheric Dispersion Modeling Resources (Mazzola and Addis 1995). A list of European heavy-gas codes was available from another survey (Hanna 1995). Additional codes were recommended for review by APAC's Spills Working Group (Brereton 1995), members of the CDCA Working Group, and DOE and EPA representatives. The initial list of the 135 codes identified is given in Table E. 1 of Appendix E.

Since the resources available to the working group for code scientific review were limited, the list had to be substantially reduced before detailed evaluations were started. Selection criteria were established to narrow the array of codes to be considered, and the codes selected were then classified into two "tiers." Tier I codes would receive a full review and would be used 
to run a set of test cases. Tier II codes would be reviewed, but test runs would not be made. The codes not included in Tier I or $\square$ were not to be reviewed during this phase of the APAC methodology evaluation project. A few of the excluded codes were recommended for future review, either because they were not yet fully developed, ware proprietary, or were discovered too late for review. The selection criteria used to reduce the number of codes to be reviewed were as follow:

1. Codes not used for accidental releases of chemicals to the atmosphere were excluded.

2. The code had to be publicly available and portable for use in making test runs (Tier I requirement).

3. Codes that were written at DOE facilities but were not currently in use and supported were excluded. This group included many older codes that had been replaced.

4. Source code and documentation had to be available, 12

5. Codes used solely for research applications were excluded.

6. Developers of proprietary models generally prohibit access to their source codes; therefore, such models were excluded from consideration at this time.

7. It was desirable, but not required, that the code could be run on a PC.

8. It was preferred, but not required, that the code have an interface suitable for use with a chemical source term and provide results in a form suitable for comparison with guideline toxic limits (a code designed for radiological source terms alone, for example, would not qualify).

9. All codes evaluated in detail and recommend for CDCA Working Group review by the APAC Spills Working Group were identified for Tier I model review.

Application of these nine criteria reduced the list of 134 codes to the 35 models identified in Appendix E. During the second CDCA Working Group meeting, these 35 models were subjected to a more intense screening and were separated into Tier I and Tier II lists. As additional information became available, further adjustments were made. In addition, since several EPA codes were on the original list, EPA representatives were asked to recommend the

12 In some instances, the source code was not obtained for the purpose of Tier I model review and evaluation. In those instances it was the understanding of the reviewer that upon request the sources code would be made available. 
more appropriate ones for review. The final list of 24 codes selected for evaluation is provided in Appendix $E$ and in Tables 3.1 and 3.2. Features of the individual codes are summarized in Sections 3.3.1 (Tier I codes) and 3.3.2 (Tier II codes).

\subsection{SUMMARY OF CODES SELECTED}

\subsubsection{Tier I Codes}

A profile of each Tier I code selected for review by the working group (see Table 3.1) is provided below. The profiles include a brief statement on the model's capabilities, followed by capsule descriptions of its limitations and key areas of applicability. The descriptions are not

\section{TABLE 3.1 Summary of Tier I Codes Selected for Review by the CDCA Working Group}

\begin{tabular}{|c|c|c|}
\hline Code Name (Version) & Developer/Sponsor & $\begin{array}{l}\text { Additional Factors Considered for } \\
\text { Assigning a Tier I Designation }\end{array}$ \\
\hline $\operatorname{ADAM}(2.1)$ & Phillips Labs/U.S. Air Force & $\begin{array}{l}\text { Spills Working Group recommendation, models } \\
\text { reactivity of } \mathrm{HF} \text { and } \mathrm{N}_{2} \mathrm{O}_{4}\end{array}$ \\
\hline $\mathrm{ALOHA}^{3}(5.2)$ & NOAAVEA & Spilis Working Group recommendation \\
\hline CALPUFF (3.0) & Earth TechEPA & Suggested by EPA for review \\
\hline CASRAM-SC $(0.8)$ & $\begin{array}{l}\text { ANL \& University of } \\
\text { Illinois/DOT \& DOE }\end{array}$ & Spills Working Group recommendation \\
\hline $\operatorname{DEGADIS}^{\mathrm{b}}(2.1)$ & Liniversity of AKJEPA & EPA supported \\
\hline FEM3C $(3.0)$ & LLNL/U.S. Amy & Able to handle complex 3-D flow fields \\
\hline HGSYSTEM $^{\mathrm{b}}(3.0)$ & Shell Research Ltd.API & $\begin{array}{l}\text { Spills Working Group recommendation, detailed } \\
\text { treatment of hiF chemistry and thermodynamics }\end{array}$ \\
\hline HOTMACRAPTADC $(1996)$ & LANLUS. Air Force & $\begin{array}{l}\text { Prognostic meteorological model able to handle } \\
\text { complex 3-D Elow fields (run on SPARC work } \\
\text { station) }\end{array}$ \\
\hline INPLFF (2.3) & EPAVEPA & Suggested by EPA for review \\
\hline SCIPLTF $(0.338)$ & ARAPDSWA & Accounts for concentration fluctuations \\
\hline SLAB ${ }^{a, b}(1990)$ & LLNL/DOE & Spills Working Group recommendation \\
\hline TSCREEN (94133) & EPA/EPA & $\begin{array}{l}\text { Spills Working Group recommendation and } \\
\text { suggested by EPA for review }\end{array}$ \\
\hline VLSTRACK (1.6) & NSWCJU.S. Navy & $\begin{array}{l}\text { Specific to chemicalbiological agents of military } \\
\text { interest }\end{array}$ \\
\hline
\end{tabular}

a Used in preparation of "look-up" tables for use in preparation of Risk Maragement Plans for compliance with EPA's Chemical Accident Prevention Rule (EPA 1996a).

b Referenced as an alternative "Appendix B" model in EPA's Guidance on Air Quality Models (GLAQM) (EPA 1996b). Alternative rnodels, in regulatory applications, can be used in situations were the preferred model (Appendix A of GLAQM) is not applicable. Models must meet the following conditions for approval in regulatory applications as "Appendix B" models:

i. the model can be demonstrated to be applicable to the problem on a theoretical basis;

ii. the data bases that are necessary to perform the analysis are available and adequate; and

iii. performance evaluations of the model in similar circumstances have shown that the model is not biased toward underestimates.

" The proprietary version of HOTMACRR.APTAD (Yamada 1988) has been approved by EPA as an altemate "Appendix B" model. 
intended to provide a comprehensive listing of a particular code's attributes or limitations. The significance of a model's limitations would depend upon the constraining conditions of a particular application. Additional information is provided in Appendix $F$, which discusses the detailed reviews and evaluations of each model. In addition to the requirements listed in Section 3.2, key considerations in sclection of Tier I codes are noted in Table 3.1.

\section{ADAM (Air Force Dispersion Assessment Model) - Version 2.1}

Capabilities: ADAM an interactive PC-based model that incorporates a source-term (emissions simulator) that treats thermodynamics and chemistry of eight chemicals of interest to the Air Force (ammonia, nitrogen tetroxide, hydrogen sulfide, hydrogen fluoride, fluorine, sulfur dioxide, chlorine, and phosgene). The dispersion model treats neutral or dense jets and ground-based dense vapor clouds and provides a transition to a standard passive gas plume. The code is consistent with the AFTOX model solution for passive gas plumes. Emissions are simulated as continuous or instantaneous releases (liquid, vapor, or twophased) that include jets from pressurized or unpressurized containers, as well as evaporative emissions from liquid spills. ADAM accounts for simple chemical and phase changes.

Limitations: The code's chemical library is limited to eight chemicals; it has restricted capabilities for handling other chemicals of interest. (Adding chemicals to ADAM's physical-chemical property database is possible, but not a trivial exercise.) It should be noted that if the release scenario involves an elevated release or a vertically pointing jet, the code will assume the worst-case situation of a ground-based horizontal jet. If multiple simulations are required they must be done one at a time with user input control during model simulations.

Use: $\quad$ ADAM is applicable to ground-level releases of the eight chemicals mentioned above and to situations involving jets pointing horizontally. It is very useful in computing release rates for those chemicals and estimating dispersion in situations involving phase and chemical changes (considers $\mathrm{N}_{2} \mathrm{O}_{4}-\mathrm{NO}_{2}, \mathrm{HF}$, and $\mathrm{NH}_{3}$ chemistry).

\section{ALOHA (Areal Locations of Hazardous Atmospheres) - Version 5.2}

Capabilities: ALOHA is a PC-based, menu-driven model (employs a graphical user interface [GUI]), primarily intended as an emergency response model for rapid deployment by responders, as well as for use in emergency planning. It incorporates source strength algorithm with three release options: (1) pool evaporation, (2) liquid/gas leaks from tank, and (3) gas leaks from ruptured pipe. It handles both passive (Gaussian) and heavy-gas (ALOHA-DEGADIS miodel) dispersion. ALOHA's output is both in text and graphic form and includes a "footprint" plot of the area downwind of a release where concentrations may exceed a user-set threshold level. ALOHA can accept weather data transmitted from portable monitoring stations and can plot footprints on electronic tnaps 
displayed in a companion mapping application (MARPLOT). ALOHA computes time-dependent source strength for evaporating puddles (boiling or nonboiling), pressurized gas, nonpressurized gas, or liquid release from a storage vessel, and pressurized gas from a pipeline. The user can also enter a constant source strength and release duration. The ALOHA model incorporates physical and thermodynamic properties of 947 pure chemicals obtained in part from the Design Institute for Physical Property Reporting (DIPPR) database.

Limitations: ALOHA does not account for either momentum or buoyancy plume rise for neutrally buoyant releases. For dense-gas releases, it does not account for the elevation of the release and assumes it is a ground-level release. Aerodynamic effects from nearby buildings are not accounted for, while any type of deposition (e.g., gravitational settling, dry deposition, precipitation scavenging) is beyond the model's scope. Although ALOHA has a large chemical library, it cannot model chemical mixtures or account for chemical transformations in the atmosphere during transport to a receptor. If multiple simulations are required, they must be run one at a time with the user controlling the input as simulation progresses.

Use: $\quad$ ALOHA is best suited for emergency response applications, inclusive of use in the field by an on-scene coordinator. It is particularly well suited to applications involving ground-level releases of dense gases away from topographic and building obstacles. Its extensive chemical library and ability to treat a variety of chemical release scenarios makes ALOHA a practical chemical dispersion model.

CALPUFF (formally the California PUFF model) - Version 3.0 (Mod. 4)

Capabilities: CALPUFF is a multilayer, multispecies, non-steady-state puff dispersion model that can simulate the effects of time- and space-varying meteorological conditions on pollutant transport, transformation, and removal. The model can use either the three-dimensional meteorological fields computed by the CALMET preprocessor or simple single-station winds. CALPUFF contains algorithms for near-source effects such as building downwash, a transitional plume rise, partial plume penetration, sub-grid-scale complex terrain interactions, as well as longer-range effects such as pollutant removal (wet scavenging and diy deposition), chemical transformation, vertical wind shear, and overwater transport. The model has mesoscale capabilities that are normally not required for chemical spill analyses. Most of the algorithms contain options to treat the physical processes at different levels of detail, depending on the model application. Time-dependent source and emission data from point, line, volume, and area sources can be used. Multiple options are provided for defining dispersion coefficients. Time-varying heat flux and emissions from controlled bums and wildfires can be calculated with an interface to the Emissions Production Model (EPM). A GUI includes point-and-click model setup and data entry, enhanced error checking of model inputs, and on-line help files. 
Limitations: Although CALPUFF is comprehensive, it was not designed for simulating dense gas dispersion effects or for use in evaluating instantaneous or short-duration releases of hazardous materials. The model is best applied to continuous releases from industrial combustion sources rather than short-duration releases typical of many chemical spills. Averaging times are long (minimum of one hour) for input data (release rate and meteorology) and output. CALPUFF does not have a builtin library of chemicals or a spills front end.

Use: $\quad$ CALPUFF is primarily applicable to continuous release durations for industrial sources in complex meteorological and terrain situations, including multiple emissions from multiple source locations. CALPUFF may be used for certain specific DOE applications involving larger releases when long-range transport is important. Also, it can be used to model the effects of fires. The upgrades included in the newly released Version 5.0 (Mod. 5) of CALPUFF are described in Appendix F. Version 6 (Mod. 6), which has provisions for time steps of less than one hour, should be available for release by the end of 1997 or the beginning of 1998.

CASRAM-SC (Chemical Accident Stochastic Risk Assessment Model - Single Case)

Capabilities: CASRAM-SC is a comprehensive model that includes modules to simulate source emissions (including pool evaporation) and transport and dispersion. The model contains a surface energy method for parameterizing winds and turbulence near the ground. Its chemical database library contains physical properties (seven incorporated, three of which are temperature dependent) for 190 chemical compounds obtained from the DIPPR database. The pertinent physical property data for any of the over 900 chemicals in DIPPR can be incorporated into the model, as needed. with relative ease. The model computes hazard zones and related health consequences. An option is provided to account for the accident frequency and chemical release probability from transportation of hazardous material containers. When coupled with preprocessed historical meteorology and population densities, the model is capable of providing quantitative risk estimates.

Limitations: The model is not capable of simulating dense-gas behavior. The version eyaluated did not have a users guide, and the available technical documentation was preliminary. Although some minor bugs were detected in the reviewed code, the developer has given assurance that these issues have been eliminated in the current version of the code.

Use: $\quad$ CASRAM is capable of simulating a widc variety of release scenarios but is particularly well suited to assessing heaith consequence impacts and risk. 


\section{DEGADIS (Dense Gas Dispersion) -- Version 2.1}

Capabilities: DEGADIS models the atmospheric dispersion of elevated or ground-level, areasource, denser-than-air gas (or aerosol) contaminants released with either negligible momencum or as a jet from pressure relief valves into an atmospheric boundary layer over flat, unobstucted terrain. The model simulates continuous, instantaneous, or finite-duration, time-variant gas and aerosol releases. It is capable of handling the dispersion processes that accompany the gravity-driven flow, contaminant entrainment into the atmospheric boundary layer, and subsequent downwind travel from the release. DEGADIS also accounts for ground reflection when the plume's lower boundary reaches the ground level.

Limitations: DEGADIS does not provide for horizontally directed jet releases. Terrain is assumed to be flat and unobstructed. Applications of the model should be limited to releases where the depth of the dispersing layer is much greater than the height of the surface roughness elements. For transient contaminant releases where the release duration is shorter than the averaging time associated with hazardous concentration levels (e.g., 10 minutes for a short-term exposure limit), DEGADIS does not provide for concentration time averaging.

Use: DEGADIS simulates atmospheric dispersion of denser-than-air gas (or aerosol) contaminants released with negligible momentum; or as a jet, into an atmospheric boundary layer over flat, unobstructed terrain. The model treats the dispersion processes that accompany the gravity-driven flow, contaminant entrainment into the atmospheric boundary layer, and subsequent downwind transport. DEGADIS is best suited for chemical consequence assessments involving dense-gas hazardous chemical releases. It can also be applied for hazard assessments and for the development of safety basis documentation.

FEM3C (Finite Element Model, Three-Dimensional) - Version C (1994)

Capabilities: FEM3C is a three-dimensional, finite-element model designed to calculate the atmospheric dispersion of dense-gas releases. The computation approach is based on a solution of the fully three-dimensional time-dependent conservation equation of mass, momentum, energy, and chemical species. The code also possesses the option for solving two-dimensional problems, which, for analyses where such an approach is applicable, can result in large savings in computational response. The code can also model both isothermal and nonisothermal dense-gas releases, as well as neutrally buoyant vapor emissions; treat multiple simultaneous sources of instantaneous, continuous, and finiteduration releases; and model the effects of obstructions to flow and complex terrain on the vapor concentration field. The user has the option of modeling turbulence parameterized via the $\mathrm{K}$-theory or solving the $\mathrm{k}-\mathrm{E}$ transport equations. Finally, a local thermodynamic equilibrium submodel with temperaturedependent physical properties is available for analyzing dispersion scenarios involving phase-change between liquid and vapor state. 
Limitations: The FEM3C code cannot accept typical vapor/aerosol source terms (e.g., pressurized jets, time-varying vapor emissions). Furthermore, although the code can treat complex terrain (ground elevation profile), it is difficult to model the presence of heterogeneous vegetation coverage (such as grassland, bushes, and tall trees all in the same computational domain). The aerosol model is incomplete in that it does not model all the relevant physical behavior (e.g., droplet evaporation, rainout). FEM3C is configured specifically for the Cray-2 (a parallel-processing computer), and, therefore, porting the code to another mainframe architecture or serial computing hardware (e.g., workstations) would require a large computing effort (see note on workstation version of code, Section 6.3.1.3). Finally, significant postprocessing of the output by the user is required to obtain concentration values at specific locations and averaging times.

Use: $\quad$ FEM3C is appropriate in modeling vapor dispersion in the presence of complex terrain and obstacles to flow. The source types should be limited to instartaneous or constant-rate releases of vapor or vapor/liquid mixtures.

\section{HGSYSTEM (Heavy Gas System) - Version 3.0}

Capabilities: HGSYSTEM simulates releases and atmospheric dispersion of dense, neutral, and buoyant gases, with special considerations for hydrogen fluoride (HF). A separate version of the code, HGSYSTEM/UF 6 , was developed for situations involving $\mathrm{UF}_{6}$ releases. ${ }^{13}$ Physical and thermodynamic properties for 30 chemicals are incorporated in the model's database (DATAPROP). The model enables simulation of (1) ground-based transient releases of a single/multicomponent liquid or vapor jets (SPLL/HFSPLL), (2) near-source jet and elevated plume dispersion (AEROPLUME/HFPLUME), (3) pool evaporative emissions of single- or multicomponent vapor (LPOOL), (4) HF chemistry and thermodynamics (HFCT), (5) ground-based continuous steadystate or transient dense gas dispersion (HEGADAS-S/T) and instantaneous dense gas releases (HEGABOX), and (6) passive Gaussian dispersion (PGPLUME). These modules have been modified in the HGSYTEM/UF 6 version to handle $\mathrm{UF}_{6}$ chemistry/ thermodynamics and plume exothermic lift-off. The HEGADAS-S/T module includes transition from heavy gas to neutrally buoyant dispersion.

Limitations: The code's chemical library is limited to 30 chemicals, with restricted capabilities for handling other chemicals of interest. Adding chemicals to HGSYSTEM's physical-chemical property database is possible but resource intensive. The model cannot simulate flow from a pipe.

13 The $\mathrm{UF}_{6}$ version is available from Lockheed Martin Energy Systems in Oak Ridge, Tennessee, or from Earth Tech in Concord, Massachusetts (see p. 1-4 for contact address). 
Use:

HGSYSTEM is particularly well suited to applications involving HF reactive releases. and to nonreactive multicomponent releases. It is also applicable to single-component nonreactive releases of 28 other compounds or mixtures of these compounds. The code lends itself weli to predicting dispersion in the near field or in situations where temperature dependence in thermodynamic properties is important.

HOTMAC/RAPTAD (Higher Order Turbulence Model for Atmospheric Circulation/Random Particle Transport and Diffusion) - Version 1996

Capabilities: HOTMAC is a three-dimensional hydrodynamic windflow model coupled to RAPTAD, a Lagrangian diffusion model. HOTMAC solves a three-dimensional time-dependent set of conservation equations that describe wind components, potential temperature, moisture, turbulence length scale, and turbulent kinetic energy. The equations account for advection; Coriolis effects; turbulent heat, momentum, and moisture transport; and viscosity. The system treats diumally varying winds, such as the "land-sea breeze and slope winds. The code models solar and terrestrial radiation, as well as the drag and radiation effects of a forest or urban canopy, and solves a thermal diffusion problem for the soil. RAPTAD is a Monte Carlo random-particle statistical diffusion code. Pseudo particles are transported with a combination random-walk/puff dispersion approach with instantaneous velocities that include the mean wind field and the turbulence velocities.

Limitations: The greatest limitations with respect to application of HOTMAC/RAPTAD to SAR analyses are (1) spatial resolution may be too course, (2) the meteorological and terrain input data requirements are very extensive, (3) there is a current lack of adequate program user guidance and program diagnostics, and (4) no options are available for modeling denser-than-air dispersion. The vertical resolution is generally adequate (4-m increments); however, dispersion results are only reliable for a horizontal resolution of approximately $1 \mathrm{~km}$.

Use: $\quad$ HOTMAC/RAPTAD is most applicable to the transport and dispersion of a large-scale chemical release where effects of complex terrain and time-varying meteorological conditions are to be considered.

INPUFF (Integrated Puff) - Version 2.3

Capabilities: INPUFF is a Gaussian integrated puff model with a wide range of applications. The implied modeling scale is from tens of meters to tens of kilometers. The model can be used to address the accidental release of a substance over several minutes or to model the more typical continuous plume from a stack. Computations in INPUFF can be made for a multiple point source at up to 100 receptor locations. In practice, however, the number of receptor locations should be kept to a minirnum. INPUFF is primarily designed to mode! a single event during which one meteorological transition period may occur, such as going from 
aftemoon to evening conditions. Up to 144 separate meteorological periods of the same length may be used to characterize the meteorology during the event. This capability provides a time resolution that ranges from minutes to an hour. The user has the option of specifying the wind field for each meteorological period at up to 100 grid locations or allowing the model to default to a homogeneous wind field.

Three dispersion algorithms are incorporated within INPUFF, two as user selected options. The user has a choice for dispersion over short travel times, the traditional Pasquill-Gifford (P-G) scheme (Tumer 1970) or a similarity based approach described by Irwin (1983). The later approach, which requires specification of the variances of the vertical and lateral wind direction, is a synthesis of work performed by Draxler (1976) and Cramer (1976). Over long travel times, the model defaults to a dispersion scheme in which the growth of the puff becomes proportional to the square root of time. Optionally, the user can incorporate a subroutine of his choice for estimating atınospheric dispersion.

INPUFF utilizes the deposition algorithms given by Rao (1982). In the limit when pollutant sertling and dry deposition velocities are zero, these expressions reduce to the Gaussian diffusion algorithrns.

Limitations: Use of the code is limited to modeling the neutrally buoyant releases of passive gases in uniform terrain. No chemical library or source-term algorithm is provided. Other limitations not necessarily unique to NPUFF, include the inability to address (1) chemical reactions, (2) complex terain, and (3) building downwash.

Use: $\quad$ Although NPUFF has a wide range of applications, it is best used in situations that require considering temporally varying source emissions and temporally and spatially varying wind fields to simulate concentrations at multiple release and receptor locations.

SCIPUFF (Second-Order Closure Integrated Puff) — Version 0.338

Capabilities: SCIPUFF simulates atmospheric transport and dispersion of neutral and buoyant vapor clouds under complex flow regimes. The model employs a terrainfollowing coordinate system so that dispersion over complex terrain can be modeled. Concentration fields can be estimated on local to continental scales. In addition to calculating the mean vaiue of the concentration field, SCIPUFF provides estimates of concentration fluctuations by accounting for the stochastic properties of the atmosphere (e.g., wind fluctuations). The model can provide a probabilistic description of dispersion results that gives a quantitative characterization of the reliability of the model predictions. 
Limitations: The version evaluated is not capable of simulating the release and dispersion of dense vapor clouds. Model output is restricted to graphical displays, with no option for producing tabular predictions. Although not a specific limitation of SCIPUFF per se, its source-term front end (HASCAL 1.0) neither simulates release-rate physics typically encountered in rapid chemical releases from pressurized jets nor contains a physical-chemical property database to account for source release thermodynamics.

Use: The model is useful in applications requiring concentration simulations from non-dense-gas releases in complex terrain or in applications where meso- and synoptic-scale phenomena are important. Situations requiring an estimate of the uncertainty in model predictions are particularity well suited to the strengths of SCIPUFF.

\section{SLAB (Slab Model) - Version 1990}

Capabilities: SLAB is a computer model that simulates the atmospheric dispersion of denser-than-air releases and includes air entrainment into heavy gas cloud. It can treat continuous, finite duration, and instantaneous releases from four types of sources: a ground-level evaporating pool, an elevated horizontal jet, a stack or elevated vertical jet, and a ground-based instantaneous release. The evaporating pool source is assumed to be pure vapor in accordance with the evaporation process. All of the remaining sources may be either pure vapor or a mixture of vapor and liquid droplets. Although the model is primarily designed to treat denser-than-air releases, it will also simulate cloud dispersion of neutrally buoyant releases and includes lofting of the cloud if it becomes lighter than air. The model is capable of simulating (1) finite duration releases, including an instantaneous release using transient puff dispersion calculations; (2) elevated horizontal and vertical jet source releases; and (3) the thermodynamic treatment of liquid droplet formation and evaporation of both the ambient water vapor and the released emission.

To simplify the solution of the conservation equations, the equations are spatially averaged with the cloud modeled as either a steady-state plume or a transient puff. SLAB applies to continuous, instantaneous, and transient releases. A continuous release (very long source duration) is treated as a steady-state plume. In the case of a finite duration release, cloud dispersion is initially described using the steady-state plume mode and remains in the steady-state plume mode as long as the source is active. Once the source is shut off, the cloud is treated as a transient puff, and subsequent dispersion is calculated using the puff mode. For an instantaneous release, the transient puff dispersion mode is used for the entire calculation. Solution of the spatially averaged conservation equations in either dispersion mode yields the spatially averaged cloud properties. 
Limitations: Source emission estimates must be made independently by the user. Other limitations, not necessarily unique to SLAB, include the inability to address (1) chemical reactions, (2) complex terrain, and (3) building downwash.

Use: $\quad$ SLAB is particularly useful in providing time-averaged concentrations, as a function of travel time, and three spatial dimensions: downwind distance, crosswind distance, and beight above ground. The predicted concentrations take into account the effects of plume meander, the finite duration of the release, and the length of the averaging time. A long list of users have tested it successfully over a broad range of input conditions. The EPA used SLAB (and ALOHA) to develop the "look-up" tables in the agency's 1996 Risk Management Plan (RMP) rulemaking.

\section{TSCREEN (Toxic SCREEN) - Version 94133}

Capabilities: TSCREEN is designed to analyze neutrally buoyant and heavier-than-air toxic emissions and their subsequent dispersion from 1 of 27 possible types of releases. The model implements the procedures in the Workbook of Screening Techniques for Assessing Impacts of Toxic Air Pollutants (EPA-454/R-92-024). Four different transport/dispersion modules are included to account for a variety of instantaneous and continuous release configurations, including point and area sources. The modules are SCREEN2, RVD, PUFF, and the Britter-McQuaid model. TSCREEN automatically selects and executes the appropriate dispersion model for the scenario.

SCREEN2 is a Gaussian dispersion model applicable to continuous releases of particulate matter and nonreactive, nondense gases that are emitted from point, area, volume, and flared sources. The module implements all of the singlesource, short-term procedures contained in the EPA screening procedures document. It provides estimated maximum ground-level concentrations and distances to the maximum based, on a preselected range of meteorological conditions. In addition, SCREEN2 has the option of incorporating the effects of building downwash. The PUFF model is used when the release duration is finite but smaller than the travel time (i.e., an instantaneous release). This model is based on the Gaussian instantaneous puff equation and is applicable for naturally buoyant, nonreactive, toxic air releases.

The RVD module estimates short-tenm ambient concentrations for screening pollutant sources emitting denser-than-air gases and aerosols through vertically directed jet releases, such as holes in pressurized containers. The model is based on empirical equations derived from wind tunnel tests and estimates the maximum ground-level plume concentration at up to 30 downwind receptor locations. The Britter-McQuaid model is used for continuous and instantaneous releases of denser-than-air gases from liquid sources. 
Limitations: Toxic air contaminants are assumed to be nonreactive and nondepositing, and, therefore, the model is not applicable to reactive gases and particle depositions. For two-phase flows, all released liquid is assumed to travel downwind as an aerosol, with insignificant rainout near the source. Denser-than-air contaminant behavior is a consequence of the initial contaminant density, the contaminant release rate, and the ambient wind speed. Meteorological conditions resulting in worst-case concentrations cannot be uniquely defined. Time-dependent emissions cannot be simulated. Releases are assumed to be steady and under ideal conditions for gas and liquid flows.

Use: The program is designed as a screening tool to define the hazard level associated with chemical spills and to determine if more refined analyses are wartanted. It is intended for use on small- to mid-scale, nonaccidental releases.

VLSTRACK (Vapor, Liquid, Solid Tracking) - Version 1.6

Capabilities:

The VLSTRACK computer model, version 1.6, provides approximate downwind hazard predictions for a wide range of chemical and biological agents and munitions of military interest. The program was developed to be "user-friendly" and portable as FORTRAN code on either a high-speed workstation or a 386/486 or equivalent microprocessor-based personal computer. VLSTRACK features (1) smart input windows that check input parameter combinations to ensure that a reasonable attack is being defined and (2) simple and informative output graphics that display the hazard footprint for agent deposition, dosage, or concentration. The model can simulate dense gas behavior. Output can be obtained either as a cumulative hazard from the time of the attack or as a periodic hazard for each time period. The model also features variable meteorology; it allows for interfacing the attack with a meteorological forecast. This feature is very important for biological and secondary evaporation computations. For quick estimates useful for preliminary hazard evaluation, the model features a rapid approximation option for each of the attack situations. The rigorous computations can then be done if a more accurate hazard estimate is required and time permits.

The model incorporates a trivariate Gaussian puff model with varying meteorological inputs. A complex terrain version (2.1) is available but was not evaluated in this study. The model is adapted for high-altitude releases and takes the density of the plume into account, although the classical slumping treatment of heavy gas near the ground is not considered to be appropriate for military applications. A bivariate Gaussian distribution equation is used to determine deposition. A calculation grid with dynamic grid spacing is used to accumulate doses from many source locations representing multiple munitions.

Limitations: Source configurations are limited to munitions, and the model contains no library of chemicals of interest to DOE. The output is not easily compared with toxic limits expressed as ppm or concentrations. Other limitations, not necessarily 
unique to VLSTRACK, include the inability to address (1) chemical reactions and (2) building downwash.

Use: VLSTRACK is designed for estimating effects from chemical/biological warheads. It is not used in the public sector and is considered to be "Critical Technology." Applications are not well publicized, but the supporting documentation does mention Operation Desert Shield with respect to an earlier version.

\subsubsection{Tier II Codes}

This section presents the profiles for Tier II models, which are listed in Table 3.2. As for the Tier I profiles given in Section 3.3.1, these profiles include brief capsules of model capabilities, limitations, and recommended use. Additional information is provided in Appendix F, which discusses the detailed reviews and evaluations of each model.

TABLE 3.2 Summary of Tier II Codes Selected for Review by the CDCA Working Group

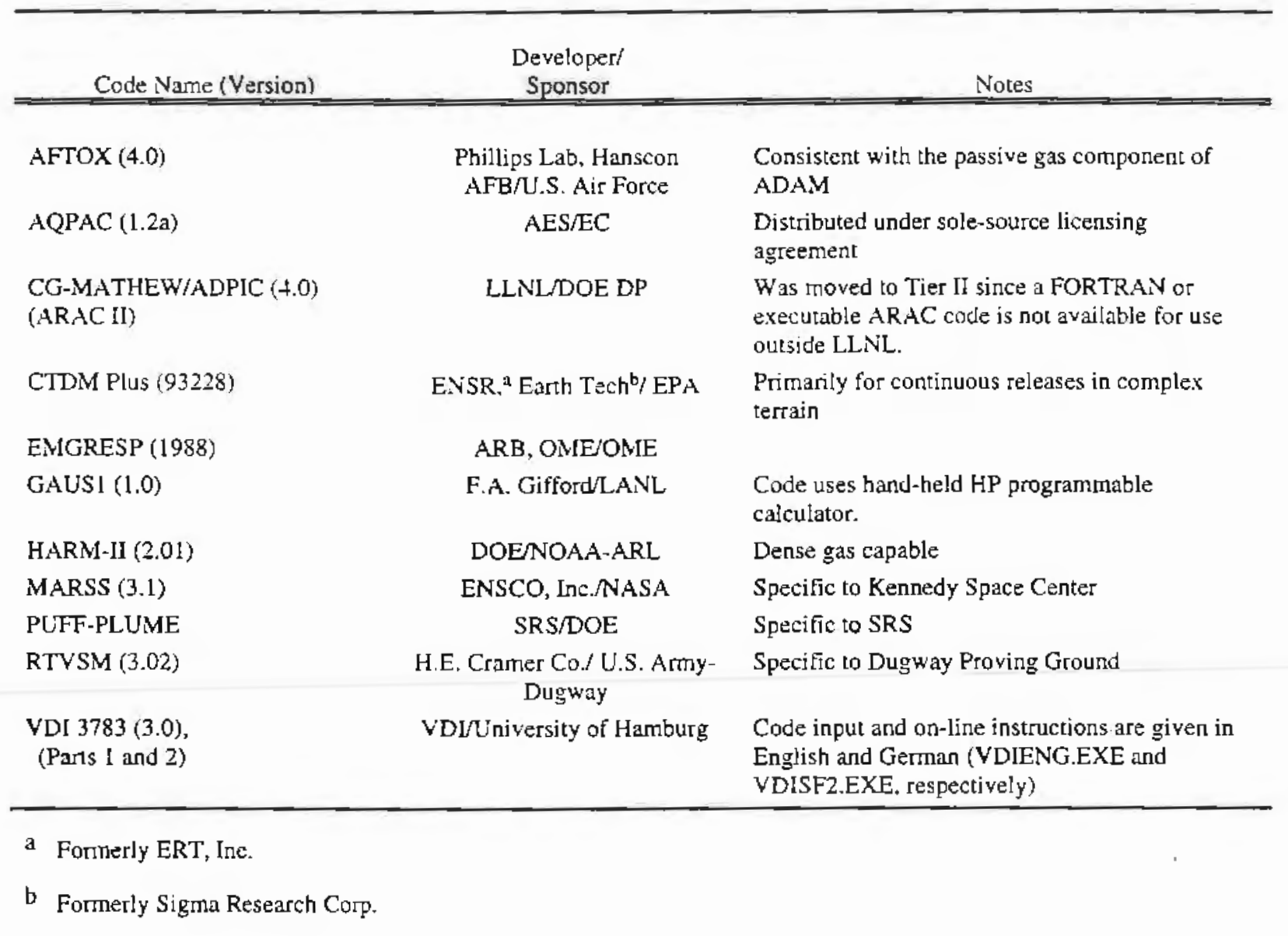


AFTOX (Air Force Toxic Chemical Dispersion Model) - Version 4.0

Capabilities: AFTOX was developed for situations where dense gas effects would not be important. The ADAM model was developed by the Air Force subsequent to the AFTOX model, and the passive gas asymptote of the ADAM model is intentionally consistent with the solution of the AFTOX model. The model contains a module for calculating the evaporative emission rate from a liquid spill. Its dispersion algorithmis can treat elevated or surface releases, neutral or buoyant plumes, and point or area sources. It contains a surface boundary layer module that allows the effects of stability and roughness to be accurately accounted for.

Limitations: The code does not apply to dense gases and cannot treat aerosols, chemical reactions, or phase changes. However, the Air Force has developed the ADAM model to treat those situations and to be consistent with AFTOX in the passive limit.

Use: - AFTOX is in use at most Air Force bases. It can be applied in an emergency response mode or in a planning mode. Although ADAM was intended to supplement AFTOX, working group members found that ADAM is not typically installed at Air Force bases, and AFTOX is being applied to all types of release scenarios.

AQPAC (Air Quality Prediction Atmospheric Code) - Version 1.2a

Capabilities: AQPAC is designed for use in emergency response to accidental releases of hazardous substances into the atmosphere. The system predicts hazard zones for potential evacuation. Both Gaussian puff and plume models are available for short- and long-term releases, respectively. A heavy-gas model is included. The model is designed for rapid field use in an emergency, runs on commonly configured PC hardware, and has a large chemical database that includes toxic limits.

Limitations: The model has a limited chemical spills front end and does not have a building wake (near-field) model.

Us $\underline{\text { Ue: }} \quad$ The code is designed primarily for use in real-time applications by the Atmospheric Enviromment Service in Canada but can be effectively used to screen the effects of most chemical releases.

ARAC II - CG-MATHEW/ADPIC (Conjugate-Gradient Mass-Adjusted Three-Dimensional Wind field/Atmospheric Diffusion Particle-in-Cell) - Version 4.0

Capabilities: This model consists of a collection of real-time meteorological data acquisition, terrain, geography, dose conversion, and chemical databases; and threedimensional diagnostic models integrated in a centralized emergency operations 
center. The system includes automated communications with remote ARAC site workstations at fixed facilities around the country that have on-site tower meteorological data acquisition. It was classified as Tier II because the code was not portable; thus reviewers could not make independent runs, and source code was not available.

Limitations: The model does not handle dense-gas releases and does not have the required physics and thermodynamics to simulate short-duration transient or steady chemical release rates. Although the model can be run independent of its several associated on-line databases and connected submodels, the breath of its capabilities would not be realized. At the time of the CDCA model reviews, CG-MATHEW/ADPIC was not available for distribution to users outside the ARAC center at Lawrence Livermore National Laboratory.

Use: The model is particularly useful in providing rapid response in situations requiring consequence assessment for major chemical and radiological events over complex terrain. Examples of such large-scale applications include the Kuwait oil fires, Chomobyl nuclear reactor accident, and Mt. Pinatubo eruption.

CTDMPLUS (Complex-Terrain Dispersion Models - Plus Algorithms for Unstable Situations) - Version 93228

Capabilities: CTDMPLUS is a refined air quality model for use in all stability conditions for complex terain applications. It is intended for application to buoyant or neutrally buoyant releases. The code calculates on an hourly (or appropriate steady averaging period) basis how the plume trajectory (and, in stable/neutral conditions, the shape) is deformed by each hill. The computed concentration at each receptor is then derived from the receptor position on the hili and the resultant plume position and shape.

Limitations: The code is limited to modeling the neutrally buoyant releases of passive gases. No chemical library or source-term algorithm is provided in the code.

Use: $\quad$ CTDMPLUS is best used for continuous releases in situations that require the incorporation of complex terrain in dispersion modeling.

\section{EMGRESP (EMergency RESPonse) - 1988}

Capabilities: EMGRESP simulates liquid spills-evaporating pool source term calculations and dispersion calculations, using gaseous or particulate release source terms. EMGRESP uses a simple neutral buoyancy Gaussian plume dispersion model and a heavy-gas dispersion model. The model was classificd Tier II primarily because it is a Canadian code that is not generally in use in the United States. 
Limitations: The dispersion parameters used in the Gaussian dispersion model generally yield overly conservative results for downwind vapor dispersion. Time-varying source terms cannot be used in the code, and only "instantaneous" dense-gas releases are modeled.

Use: $\quad$ EMGRESP is most suitable for use as a screening tool to determine if a potential chemical release warants analysis by a more refined method.

HARM-II (Hazardous Atmospheric Release Model) — Version 2.01

Capabilities: HARM-II is an interactive dose assessment model designed to predict consequences of accidental releases of hazardous materials, either chemical or radioactive. The model combines both passive- and heavy-gas codes for chemical spills, as well as standard transport and diffusion codes for radionuclide releases. Reactive chemistry is considered for those chemicals with exothermic release characteristics. The calculations are performed on a scale of 10 meters to 30 kilometers. The model considers both passive and heavy gases, as well as aerosols. HARM- $\Pi$ address both mechanical and buoyant plume risk and is a mass-consistent model with a three-dimensional wind field. The model contains algorithms that address dry deposition and gravitational settling.

Limitations: HARM-II is subject to the normal limitations of Gaussian modeling and simple heavy-gas models. It is site-specific, requiring data on local topography and building configurations. It does not consider the effects from wet deposition, and its chemical library is limited to about 266 chemicals; as contrasted with 947 chemicals in ALOHA.

Use: $\quad$ HARM-II is primarily an emergency management consequence assessment tool that addresses accidental releases of hazardous chemicals and radionuclides. Applications are geared to facilities managed by the Oak Ridge Operations Office. They include the Y-12 Plant, Oak Ridge National Laboratory, the K-25 Plant, Paducah, Portsmouth, and Femald. It can also be used for hazard assessments and environmental impact statements. It is simple enough to be used for scoping evaluations, yet because of its treatment of the physics of chemical transformations, it can be used for specialty analyses as well.

GAUS I (GAUSsian) - Version 1.0

Capabilities: The GAUS 1 code is written for a Hewlett-Packard hand-held calculator and contains more than 2,200 built-in mathematical and progranming commands. It calculates time-averaged, straight-line (unvarying winds for duration of an individual simulation) rural and urban plume concentrations using the standard Gaussian formulas that typically appear in air pollution regulatory applications. Short-duration releases can be simulated as quasi-instantaneous releases using a Gaussian puff formula. The program employs a number of very useful auxiliary 
formulas, for example, in building wake, dry deposition, dosage, and risk applications.

Limitations: The code is witten for a specific programmable calculator (HP48SX), which may restrict user access. No source release or dense-gas algorithm is included in the model formulation.

Use: $\quad$ The model is versatile and easy to use for evaluating a wide range of practical air contaminant problems.

MARSS (Meteorological and Range Safety Support) - Version 3.1

Capabilities: MARSS is a stand-alone system. Meteorological data from the Weather Information Network Display System (WINDS), effluent dispersion from REEDM, and BLAST damage assessment model outputs are obtained as ASCII files from the Cyber 860 mainframe by the microVAXes using a conmunication link. Several main processes are available: meteorology, diffusion, safety map, and auxiliary display. The diffusion process provides the paths and toxic corridors predicted by the dispersion models OB/DG and/or LOMPUFF. The major available functions are graphic weather displays, tabular weather displays, REEDM concentration displays, etc. It can make concurrent runs of up to 12 OB/DG scenarios and one LOMPUFF scenario.

Limitations: The OB/DG model is limited in its capabilities and does not fully leverage the complex array of meteorological data sources available. In addition, the model has no ability to account for vertical variations in the windfield. It is unable to deal with elevated releases and has a weak source strength submodel. The LOMPUFF model also can not account for vertical variations in the wind field.

Use: $\quad$ MARSS is exciusively used at Kennedy Space Center. However, its algorithms are applicable to other locations, as long as the limitations of the model are observed.

PUFF-PLUME (Puff and Plume Dispersion Models for Emergency Response)

Capabilities: The PUFF-PLUME code simulates chemical/radionuclide dispersion that includes wet and dry deposition, real-time input of meteorological observations and forecasts, and puff or plume dispersion modes. It is one of a suite of codes for modeling atmospheric releases and is used primarily for first-cut results in emergency sinuations.

Limitations: The code is limited to modeling the neutrally buoyant releases of passive gases in uniform terrain. No chemical library or source-term algorithm is provided in the code. Moreover, it is too site-specific. 
USe: $\quad$ PUFF-PLUME is best used in situations that require short response time for predicting the consequences resulting from accidental releases.

RTVSM (Real-Time Volume Source Model) -- Version 3.02

Capabilities: RTVSM version 3.02 is designed to calculate dosage, intravenous dosage, concentration, concentration versus time profiles, fumigation concentration, time-average concentration, vertical deposition, precipitation deposition, and gravitational deposition. The aforementioned calculations can be performed by using elevated buoyant and/or non-buoyant point, volume, area, stack, and/or line sources. Emission rates are user-specified for instantaneous, quasi-instantaneous, exponentially decaying, and continuous sources. RTVSM is used to assist in the design and analysis of chemical and biological agent stimulant and military smoke/obscurant dissemination tests. It uses site-specific dispersion algorithms (i.e., Cramer) developed from tracer studies at Dugway Proving Ground.

Limitations: RTVSM does not consider dry deposition for particles with aerodynamic equivalent diameters less than 10 micrometers. In its real-time mode, RTVSM is designed to access the real-time data file from Dugway Proving Ground's network of remote automated weather stations and would have to be modified for real-time applications at other locations.

Use: As developed, RTVSM is exclusively used at the Dugway Proving Ground, where it was intended for assessment of chemical and biological smoke/ obscurant testing.

VDI (Verein Deutscher Ingenicure) - Version 4.0

Capabilities: The VDI guidelines provide a computerized approach for modeling positively, neutrally, and negatively buoyant releases. The model treating positively and neutrally buoyant releases can handle time-dependent point, line, area, and volume sources and can calculate the downwind distance to a concentration level of concern for user-specified ambient conditions in the absence of buildings or obstructions to flow. The dense-gas model can compute the downwind distance to the lower flammability limit for a flammable vapor release and can compute the distance to transition to a neutrally buoyant vapor cloud for toxic gas releases. Estimates of the final toxic endpoint may be obtained by either running the neutrally buoyant model or, alternatively, running the program STOER, which combines both the positively/neutrally buoyant model with the dense-gas model. Finally, the dense-gas model can be used in conjunction with 25 different building and street canyon configurations to model the effects of obstacles to flow on downwind vapor concentration.

Limitations: The method used for modeling neutrally buoyant releases for line, area, and volume sources may potentially incur errors when the dimensions of the source are not small compared with the target downwind distance. The meteorological 
conditions are controlled internally in the dense-gas model to obtain worst-case and median dispersion conditions and are not available to the user for modification. Although the published guidelines are documented in both English and German, only the dense-gas computer model provides input instructions and output in English. Currently, the model does not have an algorithm for computing release rates from yapor or liquid spills.

Use: Its design for estimating the largest danger areas for a given initial release quantity or release rate (e.g., has a limited input requirement) make VDI a useful screening tool for estimating the flammable and toxic endpoints associated with initially dense-gas releases. These endpoint estimates can take into account the presence of fences, buildings, and street canyons located near the source point. 

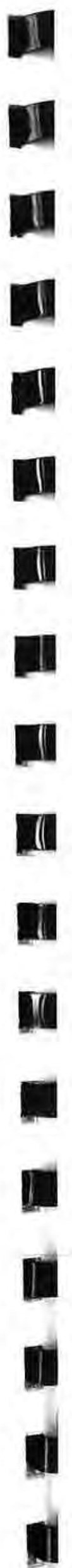


\section{BASIS FOR MODEL REVIEW AND EVALUATION}

\subsection{SUMMARY OF REQUIREMENTS AND MODEL REVIEW BASIS}

The DOE complex houses a diverse inventory of hazardous chemicals. During a recent facility survey of chemical safety practices, described in the Chemical Safety Vulnerability Report (DOE/EH-0396P) (DOE 1994), a significant number of potentially hazardous situations were revealed. Chemical safety analysis and consequence assessments for safety basis documentation (SBD) have historically not received the same level of attention given to radiological hazards. The lack of guidance in the selection and application of models in support of chemical safety analysis has lead to inconsistent assessments. These assessments have not always been appropriate for preparation of DOE non-reactor-facility Safery Analysis Reports (SARs), Basis for Interim Operation (BIO) documents, and other SBD. The results of the APAC process are intended to upgrade the level and consistency in analysis supporting SBD and be of value to DOE's Office of Defense Programs (DP), as well as to the DOE Offices of Environmental Management (EM), Environment, Safety, and Health (EH), and Nonproliferation and National Security (NN).

The contents of the requirement documents applicable to DOE facilities, summarized in Section 1.3, were used as partial guidance in establishing the multiphased code review process. Further details are provided in Appendix A. The major phases as highlighted in the model review/evaluation framework (Section 2.2) included (1) code identification and screening, (2) development of basis for model reviews, (3) development of test problems, and (4) running Tier I models with test problems and evaluating the results.

In developing the second-phase model review basis, the CDCA Working Group studied use by DOE and other federal entities of regulatory information as guidance for the selection of accident phenomenology and consequence analysis methods. The primary, but limited, guidance is found in DOE Order 5481.1B, Safety Analysis and Review System, and DOE Order 6430.1A, General Design Criteria. A second level of references includes supporting standatds (e.g., DOE Standard 1027-92). Before starting its code reviews, the CDCA Working Group developed criteria for measuring how well the current tools meet their intended objectives. The candidate chemical release methodologies were also evaluated for applicability in the context of DOE's graded approach hierarchy and in support of safety analyses as a primary function.

\subsection{DEVELOPMENT OF MODEL REVIEW WORKSHEETS}

The review bases applied to the Tier I and $\Pi$ models are both general and specific. Before codes were assigned to reviewers, a 12 -page set of model review and evaluation forms (MREFs) was developed to guide the content and level of detail expected in each code evaluation. These forms were reviewed by each working group member and revised by group consensus before use in the model reviews. A set of blank MREFs is reproduced in Appendix F. The length of each set of forms varies for each code since the reviewers were not limited as to the amount of space used for the comments. 
The MREFs are divided into two parts - Part I is for the General Model Review, and Part II is for the Specific Model Review. The contents of each part are described below.

\subsubsection{General Model Review}

Part I.A, Code Summary, of the General Model Review section lists 23 topics to be used as guidance for the reviewer in providing a descriptive summary of each code. The topics include information about where the code originated and is maintained and a summary of capabilities. An overview of applications of the model is included, along with references for model documentation, applications, and comparisons. The input and output are to be summarized and the user-friendliness assessed for setup and calculations. Specific details about hardware and software requirements are also listed. These summaries will provide potential users with information they can use at the initial screening stage of the model selection process. The model profile statements given in Section 3.3 of this report were derived from the Part L.A reviews.

The DOE uses computer codes in several ways in the process of applying a graded approach to evaluating the safety of its facilities. The standard DOE-STD-1027-92 on Hazard Categorization and Accident Analysis Techniques defines a "graded approach" as "a more rigorous and more thoroughly documented analysis and evaluation of higher-hazard facilities than lower-hazard facilities, given the potential for more wide spread and severe consequences if a higher-hazard facility fails to meet its safety basis requirements." It goes on to say that "The graded approach directs that the effort should be proportional to the complexity of the safety systems relied on to maintain an acceptable level of risk. Simple facilities would require less sophisticated analysis."

The graded approach applies to analysis classes as well as to the hazard level of a facility or hazard category. Part I.B, Evaluation Against DOE Requirements, of the General Model Review section provides a summary of how each model applies to each of five analysis classes. Table 4.1 shows each of the analysis classes considered. The evaluation forms also provide a summary of how each model relates to the analysis classes. An analyst could use this information to determine whether a particular model would be appropriate for the analysis being considered.

\subsubsection{Specific Model Review}

The specific reviews were designed to ascertain the relative scientific and technical merits and deficiencies of the codes. The results are summarized in the remaining sections of the MREFs and are used to address the detailed features of the code. The forms take on the character of questionnaires prompting the reviewer with a list of detailed code attributes (or features). Columns are provided for adding notes concerning code capabilities and limitations. The major evaluation categories considered appropriate for computer codes that address issues of atmospheric dispersion and chemical release consequences are listed below. It should be noted that the release (source term) algorithms were evaluated separately by the Spills APAC Working Group. The information requested on the evaluation forms is meant to be sufficiently detailed so that reviews done by different reviewers could be compared. 
TABLE 4.1 Examples of Analysis Classes for Five Analysis Levels

\begin{tabular}{|c|c|}
\hline Level & Analysis Class \\
\hline 1 & $\begin{array}{l}\text { Scoping Evaluations } \\
\text { - Inventory Limits } \\
\text { - Preliminary Hazard Analysis } \\
\text { - Environmental Management Hazard Category Standard }\end{array}$ \\
\hline 2 & $\begin{array}{l}\text { Hazard Analysis/Consequence Assessment } \\
\text { - Hazard Analysis Document } \\
\text { - Preliminary Functional Classification } \\
\text { - Basis for Hazard Analysis } \\
\text { - Environmental Assessment (EA) }\end{array}$ \\
\hline 3 & $\begin{array}{l}\text { Accident Analysis } \\
\begin{array}{l}\text { - SARs } \\
\text { - BIOs } \\
\text { - Final Functional Classification } \\
\text { - JCOs } \\
\text { - Environmental Impact Statement (EIS) }\end{array}\end{array}$ \\
\hline 4 & $\begin{array}{l}\text { Emergency Management/Response } \\
\text { - Basis for Protective Action } \\
\text { - Real Time Response }\end{array}$ \\
\hline 5 & $\begin{array}{l}\text { Special Purpose Analysis } \\
\text { - Hazardous Compounds/Materials Requiring Special Treatment } \\
\text { (e.g., } \mathrm{HF}_{2} \mathrm{NH}_{3}, \mathrm{UF}_{6} \text { ) } \\
\text { - Phenomenon } \mathrm{Specific} \mathrm{(e.g.,} \mathrm{complex} \mathrm{flow} \mathrm{pattems} \mathrm{induced} \mathrm{by} \text { closely space buildings, nearby terrain, seashore, lakes) } \\
\text { - Facility-based }\end{array}$ \\
\hline
\end{tabular}

Information was requested in each of the 11 categories, with several elements (code features) that were to be addressed by each reviewer. It is expected that a potential user would review these code characteristics to determine whether the code was appropriate for the intended application. In the working group review process, adjustments were made on the fonms on the basis of comments from other reviewers. Both the general and specific model review foms were distributed to the model developers for their review and comment. Any feedback form received from the developers was considered for inclusion on the final forms.

The major categories and descriptions of the kind of information requested in each are given below. Each feature had to be identified as being included or not and as being a minimum 
requirement or not, and each had to be briefly described, including limitations and assumptions. Each section also contains an "other" category for use in describing features that are not on the form. The major categories listed on the MREFs are discussed briefly below using the same alphabetic section identifiers as on the forms.

\section{A. Source Term Algorithm}

Although source term algorithms were considered separately by the Spills APAC Working Group, several of the dispersion and consequence models contain source term algorithms. The source configuration features that are available in the model are identified and briefly described. Columns are provided for indicating whether each feature of the source term was evaluated by the Spills Group and to indicate if any recommendations were made.

\section{B. Input Parameters Required to Run the Transport and Dispersion Model}

Most of the parameters in this category are used to describe the physical characteristics of the release, such as release rate, size, shape, and temperature. These characteristics are often important in initial plume dispersion and determining near-field concentrations.

\section{Dispersion Submodel Type}

The calculation model used to quantify the atmospheric dispersion is identified in this section. Several functions are listed that are used by different models, including Gaussian, gradient transport, or stochastic simulations.

\section{Model Capabilities/Physics}

The parameters included in this category are used to describe the interaction between the effluent and the physical attributcs of the ambient environment. The buoyancy (positive or negative) of the effluent relative to the atmosphere is included here. Other parameters include the effects of buildings and geographic features, wind field variations, and surface interactions.

\section{E. Transport Submodel}

The features described in this section pertain to the downwind transport of effluent in the ambient environment. Some specific examples are stochastic treatment, time scales, variable trajectory, and vertical wind profiles.

\section{F. Meteorological Input}

The meteorological data required as input to the model are identified in this section. Some or all of these data may be required by the models. There may be optional parameters in some models that can be used for more in-depth analyses. Most models do not use all of the features listed because altematives for characterizing the same phenomenon are included in some models. 


\section{G. Health Consequences Submodel}

Health Effect Wethodologies are listed in this section. These items include criteria for converting concentration into health effects and risk.

\section{H. Source/Receptor Mitigation Measures}

Mitigation strategies (e.g., evacuation, sheltering, interdiction, and application of spray/foam) that may be included in some models are described in this section.

\section{Output Capabilities}

The output (display) features of the model are described in this section. Output can be tabular or graphic in nature and can include a variety of calculated information. Models often provide data that is useful for some applications but not others; thus it is important that the user has options to reduce the volume of output.

\section{J. Uncertainty Analysis}

Uncertainty in the results of a given calculation can be expressed in different ways. This section was included to allow descriptions of the model treatment of uncertainty, if any.

\section{K. Sample Problem Results Sheet}

A cross-reference to the test problems that were run for the Tier I codes is provided in this section. The information includes which sample problems were run, a brief description of how the tests were run (and if there were difficulties), and a reference to Appendix F where the results are reproduced.

Each category had several elements that were addressed by each reviewer. It is expected that a potential user would review these code characteristics to determine whether the code was appropriate for the intended application. In the final working group meeting, adjustments were made in the sheets on the basis of comments from other reviewers. Both the general and specific model review sheets were distributed to the model developers for their review and comment.

\subsection{DESCRIPTION OF TEST PROBLEMS}

\subsubsection{Background}

Regardless of any claims in model documentation about the accuracy and applicability of a particular software program, it is prudent to make verification runs and compare model results against a standard set of test problems. Model performance evaluation is especially important when reviewing a group of models intended for use in regulatory or safety basis documentation. The best way to do this is to execute the program using data from field or laboratory experiments, and then determine the mean biases and the typical scatter of the predictions compared with the observations (e.g., see the comparative evaluation of 15 models 
using 8 field experiments reported by Hanna et al. 1993). In lieu of model performance evaluations, comparative testing (the procedure selected for these Tier I model evaluations) by running models against a set of realistic but hypothetical accident scenarios can provide useful application insights. Brighton et al. (1994) reported on a study of this type, where 8 models commonly used in Europe were run on 25 test cases. An obvious drawback to this method is that the model predictions can only be compared in a relative sense. For example, if 10 models were run for the same scenarios and 9 of those models agreed with each other within $20 \%$ while the 10th model produced results a factor of five higher, it might be that the results of the outlier model (\#10) would actually agree better with field data than would the results of the other nine. With this caveat, it was still decided to follow the test case methodology for this phase of the model evaluations and perhaps do some comparison with field data in a future phase of the study (see Section 6.4).

\subsubsection{General Rationale Behind Choice of Test Cases}

The working group determined that to evaluate the Tier I models it would be desirable to choose about 5 to 10 test cases representing a cross-section of release scenarios likely to be encountered at DOE facilities. It was decided that the cases should include situations involving evaporation from liquid spills (both cryogenic and more slowly evaporating), vertical heavy-gas jets, passive but toxic gas plumes, multicomponent dense-gas releases, and time-variable releases. The first draft of test cases was taken from the set of seven cases reported by Hanna et al. (1996) in the latest edition of the AIChE Guidelines for Use of Vapor Cloud Dispersion Models. That set was selected by an AIChE committee consisting of about 20 chemical engineers responsible for vapor cloud scenario selection, modeling, and consequence analysis within their chemical companies. The AIChE set included several pressurized jet releases involving heavygas dispersion. All but one of the release scenarios involved dense-gas behavior. This group assumed that source emissions rates were given as inputs and did not need to be calculated by the models.

It should be noted that the test problems were intended to represent classes of release types that are typically encountered in an SAR. Nonetheless, it was necessary in some cases to place physical constraints on problem definitions in order to isolate and test certain modeling features in a collection of codes. For example, consider a postulated flashing hydrogen fluoride release from a system pressure of 4 bar to atmosphere that does not initially entrain air and forms a dense, quiescent area source. In this instance, one objective of analyzing the test problem was to evaluate each code's capabilities in aerosol formation and transport modeling. Although several codes that were used to analyze this scenario possessed some capabilities in modeling vapor cloud dispersion in the presence of liquid aerosol, they differed greatly in their ability to nodel a high-momentum, two-phase jet (with capabilities ranging from a detailed threedimensional Navier-Stokes equation solver to no modeling capabilities whatsoever). Without placing some limitations on the problem statement, the final results would very likely obscure the underlying contribution to the aerosol model because of the vastly different capabilities in turbulent jet modeling. Therefore, in some cases it was advantageous to limit the conditions of the test problems in such a way that code comparisons could be made on a relative model-bymodel basis. 


\subsubsection{Description of Seven Test Cases}

Table 4.2 contains the input data sufficient to run each of the 13 Tier I models for the seven test cases. In each case, at least two sensitivity runs were also designed, involving changes to one or two input parameters, giving a total of 32 runs to make (see Table 5.3). In this evaluation, several of the models could not be run for all test cases (for example, ADAM could be run only for eight specific chemicals). A brief overview of the rationale for selection of the individual test cases is given below.

Pressurized Liquid Chlorine Spill (Test Case A): It is assumed that liquid chlorine at ambient temperature is spilled on the ground and spreads out to cover an area of $1,000 \mathrm{~m}^{2}$. Chlorine then evaporates into the atmosphere from this area source. The evaporation rate (ER) varies over a 60-minute period, as shown in Table 4.2, with a large emission rate for the first minute, followed by much smaller emission rates. The two sensitivity runs involve changes in wind speed/stability class and surface roughness.

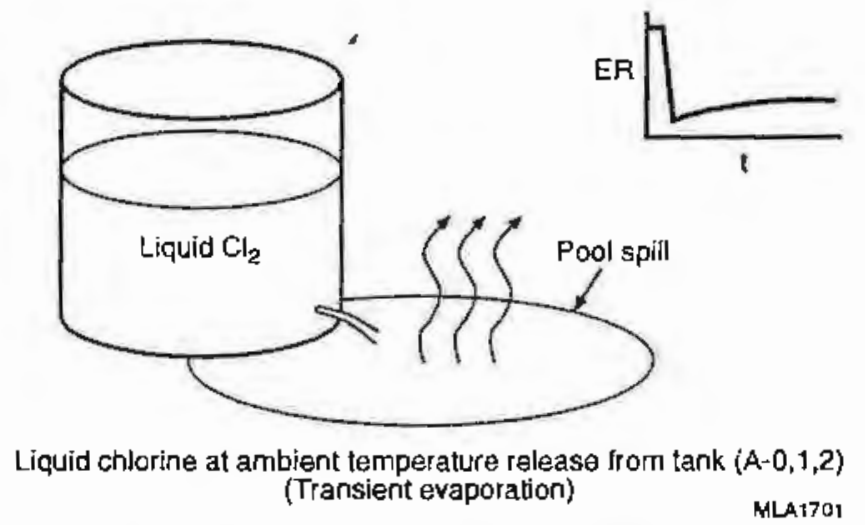

Refrigerated Liquid Chlorine Spill (Test Case B): Test case B is similar to test case A involving chlorine storage, except that the initial temperature for test case B is the boiling point of chlorine, and the evaporative emission rate is constant over one hour. The sensitivity runs involve a change in wind speed/stability and an increase in the height of the receptor.

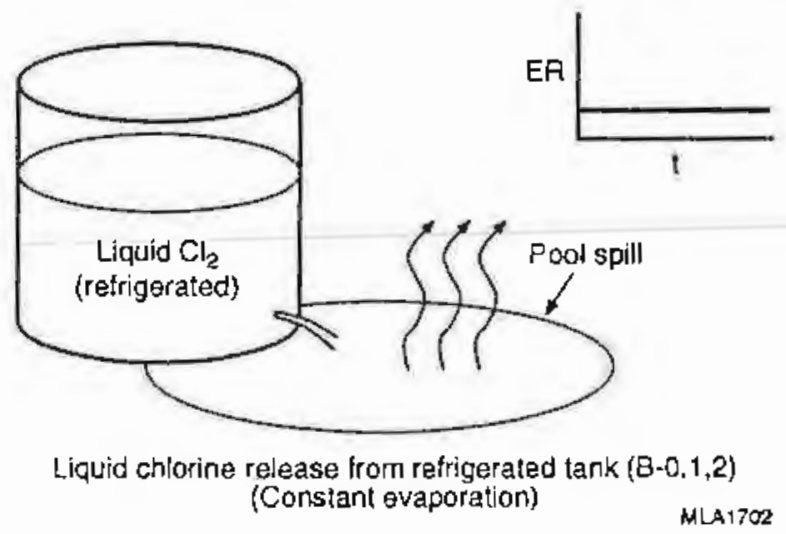


$\stackrel{f}{\infty}$ TABLE 4.2 Input/Output Specification for Accident Seenario Cases

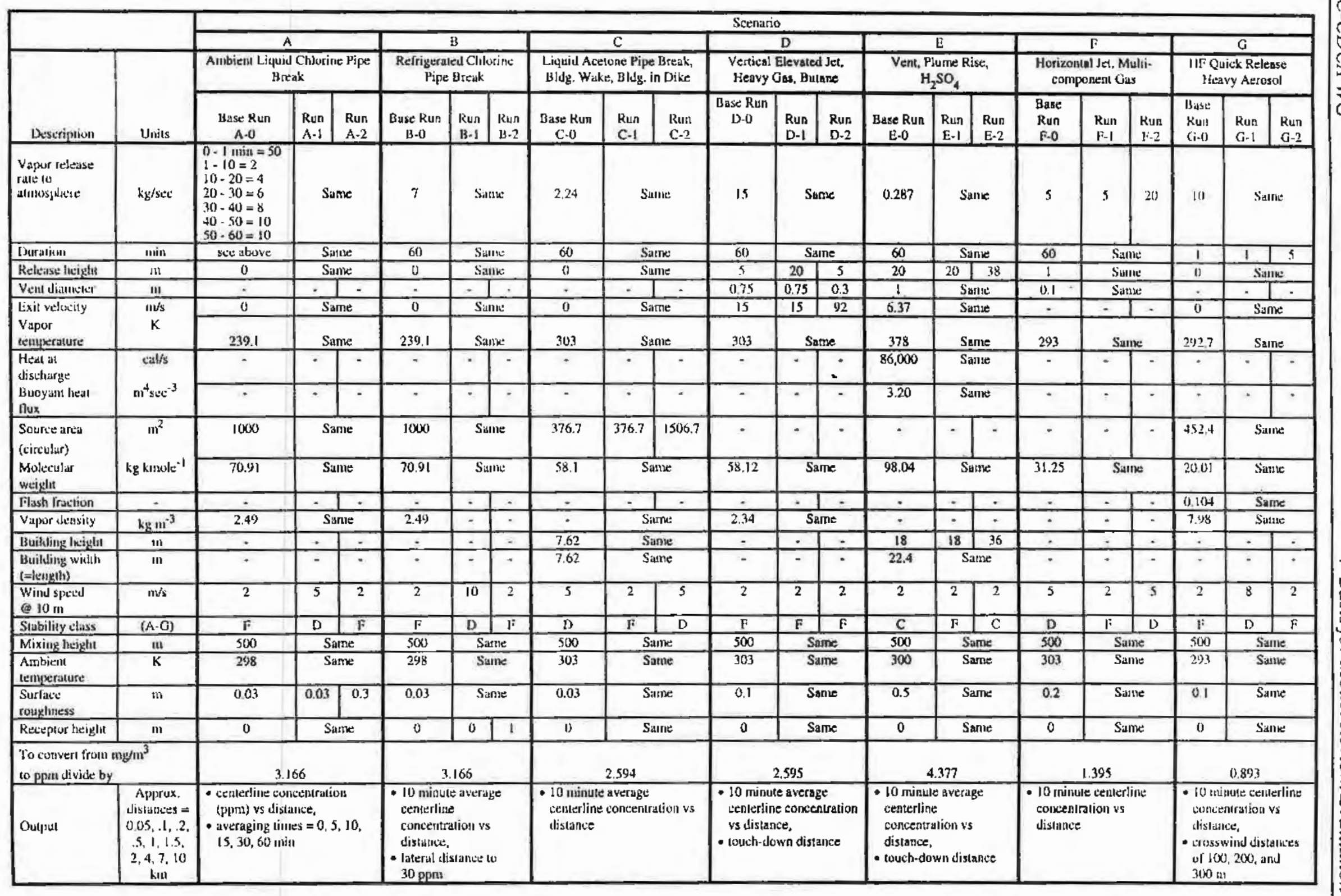


Liquid Acetone Spill (Test Case C): Liquid acetone is spilled into a diked area, with evaporation from a surface area of $376.7 \mathrm{~m}^{2}$. This test case provides a contrast to test cases $A$ and $B$, since acetone evaporates much more slowly than chlorine and the surface area of the pool is limited by the dike's geometry. Sensitivity runs include changes in wind speed/stability and surface area for evaporation.

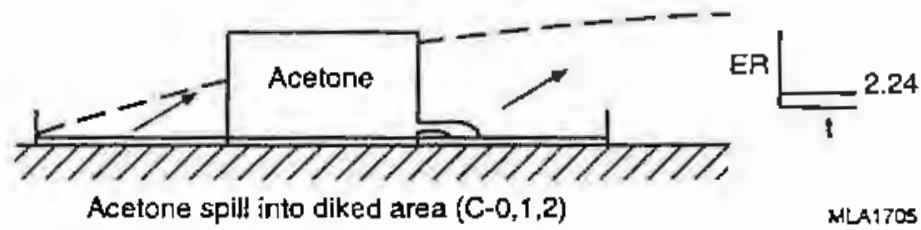

Vertical Elevated Jet of Butane (Test Case D): A dense gas, butane, is released continuously over one hour at high velocity from an upward-pointing pipe. The dense jet should initially rise because of momentum of the jet and then bend back towards the ground. Sensitivity tests involve modifications to the stack height and the initial jet diameter (which affects momentum).

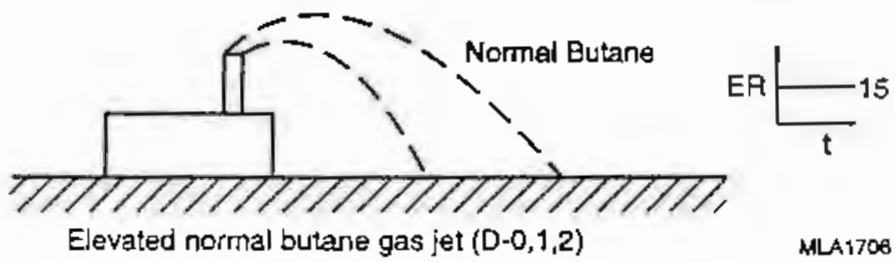

Release of Dilute Sulfuric Acid Mist from a Vent (Test Case E): It is assumed that a runaway reaction occurs in a building and resulting sulfuric acid mist is released from a vent on top of the building. The dilute plume can be treated as a slightly buoyant gas. The wind speed/stability and the building height are altered in the two sensitivity runs.

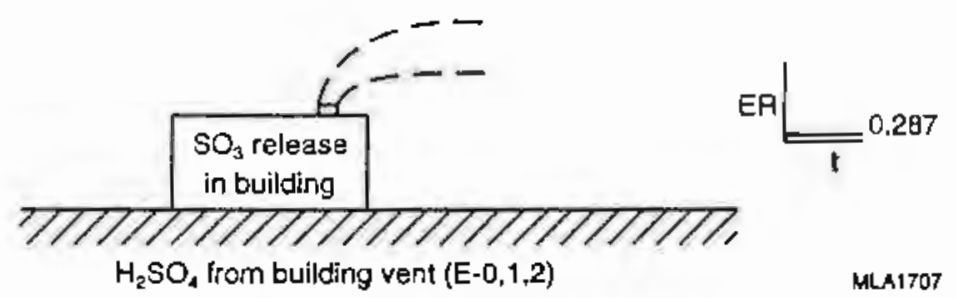

Horizontal Jet of Multicomponent Gas (Test Case F): A multicomponent gas is represented by a pseudo-gas with a molecular weight equal to the effective value of the mixture. The jet is released parallel to the ground. The sensitivity runs account for changes to wind speed/stabilicy and release rate.
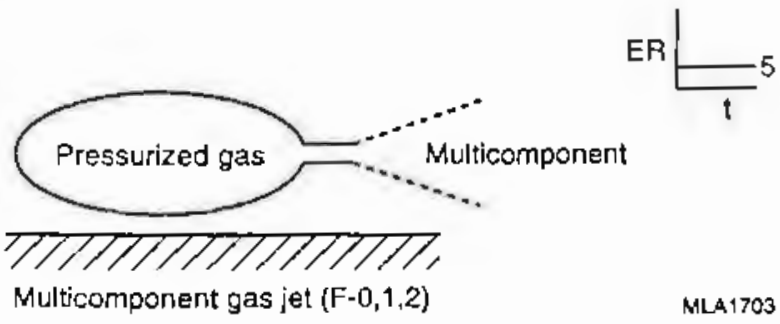
Short Duration Release of Heavy Hydrogen Fluoride (HF) Aerosol/Gas Mixture (Test Case G): Hydrogen fluoride aerosol and gas are assumed to be released at a high rate for one minute, followed by a $90 \%$ reduction for the next nine minutes. The release is simulated as an area source of area $20 \mathrm{~m}^{2}$. The wind speed/stability and duration of release are varied in the sensitivity runs.

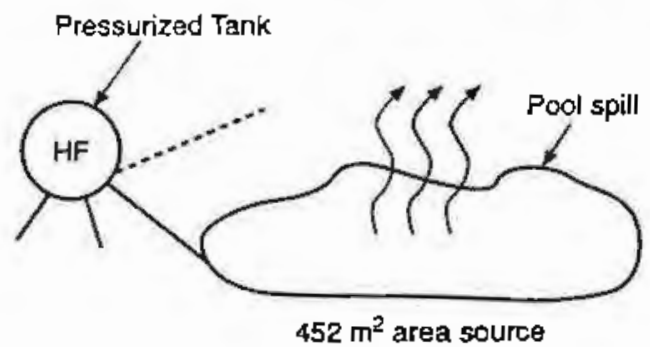

HF AerosoVGas Release (G-0,1,2)

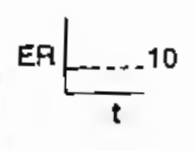

NLA1704 


\section{MODEL REVIEW AND COMPARISON RESULTS}

\subsection{REVIEW BASIS RESULTS - ANALYSIS CLASS}

The Tier I and Tier II codes were evaluated by the CDCA Working Group against the general and specific bases for review summarized in Cbapter 4 and discussed in detail in the Model Review and Evaluation Forms (MREFs) found in Appendix F. Table 5.1 indicates the applicability of the evaluated Tier I models to each of the five analysis levels shown in Table 4.1. Table 5.1 summarizes infomation from the MREFs in Appendix $F$ and represents the working group's consensus after considerable discussion.

TABLE 5.1 Programmatic Applications for Tier I Models

\begin{tabular}{|c|c|c|c|c|c|}
\hline \multirow[b]{2}{*}{ Model } & \multicolumn{5}{|c|}{ Analysis Class ${ }^{2}$} \\
\hline & $\begin{array}{c}\text { Scoping } \\
\text { Evaluations }\end{array}$ & $\begin{array}{c}\text { Hazard } \\
\text { Analysis/ } \\
\text { Consequence } \\
\text { Assessment }\end{array}$ & $\begin{array}{l}\text { Realistic } \\
\text { SAR } \\
\text { Accident } \\
\text { Analysis }\end{array}$ & $\begin{array}{c}\text { Emergency } \\
\text { Management } \\
\text { Response }\end{array}$ & $\begin{array}{l}\text { Special } \\
\text { Purpose } \\
\text { Analysis }\end{array}$ \\
\hline ADAM & $N$ & $\mathrm{Y}$ & $\mathrm{Y}$ & $\mathrm{N}$ & $\mathrm{Y}$ \\
\hline ALOHA & $\mathrm{Y}$ & $\mathrm{Y}$ & $\mathrm{Y}$ & $Y$ & $N$ \\
\hline CALPUFF & $N$ & $Y$ & $Y$ & $\mathrm{~N}$ & $\mathrm{Y}$ \\
\hline CASRAM-SC & $N$ & $\mathrm{Y}$ & $\mathrm{Y}$ & $N$ & $N$ \\
\hline DEGADIS & $\mathrm{N}$ & $\mathrm{Y}$ & $Y$ & $\mathrm{~N}$ & $\mathrm{~N}$ \\
\hline FEM3C & $\mathrm{N}$ & $\mathrm{N}$ & $\mathrm{N}$ & $N$ & $\mathrm{Y}$ \\
\hline HGSYSTEM & $\mathrm{N}$ & $\mathrm{Y}$ & $\mathrm{Y}$ & $\mathrm{N}$ & $Y$ \\
\hline HOTMAC/RAPTAD & $\mathrm{N}$ & $N$ & $\mathrm{~N}$ & $N$ & $\mathrm{Y}$ \\
\hline INPUFF & $\mathrm{Y}$ & $\mathrm{Y}$ & $\mathrm{Y}$ & $\mathrm{N}$ & $\mathrm{N}$ \\
\hline SCIPUFF & $\mathrm{N}$ & $\mathrm{Y}$ & $\mathrm{Y}$ & $\mathrm{Y}$ & $\mathrm{Y}$ \\
\hline SLAB & Y & $\mathrm{Y}$ & $\mathrm{Y}$ & $\mathrm{N}$ & $\mathrm{N}$ \\
\hline TSCREEN & $\mathrm{Y}$ & $\mathrm{Y}$ & $Y$ & $N$ & $N$ \\
\hline VLSTRACK & $N$ & $\mathrm{~N}$ & $\mathrm{~N}$ & $N$ & $\mathrm{Y}$ \\
\hline
\end{tabular}

2 See Table 4.1 for examples of applications in each of the anaiysis classes.

Because the table reflects the subjective judgment of the model reviewers, ocher, less subjective, factors should also be considered in selecting a model for a specific use. Some of the more important of these other factors are given in Table 5.2, which summarizes the key model attributes for each of the Tier I models. Table 5.2, along with reference to the MREFs and model documentation as well as the analyst's knowledge of site-specific and source-specific constraints, should be used in selecting a model for use in a particular application. 


\begin{tabular}{|c|c|c|c|c|c|c|c|c|c|c|c|c|c|}
\hline \multirow[b]{2}{*}{ Model Altribule } & \multicolumn{13}{|c|}{ Models with and without a Particular Capability or Peature ( $T=$ treased, NT = not treated) } \\
\hline & ADAM & ALOHA & CALPUFF & $\begin{array}{c}\text { CASRAM- } \\
\text { SC }\end{array}$ & DEGAIJIS" & $\mathrm{FEM} 3 \mathrm{C}$ & $\begin{array}{c}\text { HG- } \\
\text { SYSTEM }^{2}\end{array}$ & $\begin{array}{l}\text { HOTMAC } \\
\text { RAPTAD }^{b} \\
\end{array}$ & INPUFF & SCIPUFF & SLAB" & TSCREEN & $\begin{array}{l}\text { VLS- } \\
\text { TRAC } \\
\end{array}$ \\
\hline \multicolumn{14}{|l|}{ Kelease Type ${ }^{e}$} \\
\hline Spills Front End & $T$ & $\mathrm{~T}$ & NT & $T$ & $\mathrm{NT}$ & NT & $T$ & $\mathrm{NT}$ & NT & $N T^{4}$ & $\mathrm{NT}$ & $T$ & $T$ \\
\hline Pussive: Ground level & $T$ & $\mathrm{~T}$ & $T$ & $\mathrm{~T}$ & $\mathbf{T}$ & $\mathrm{T}$ & $\mathrm{T}$ & $T$ & $\mathrm{~T}$ & $\mathbf{T}$ & $\mathrm{T}$ & $T$ & $\mathrm{I}$ \\
\hline Passive: lillevaled & $T$ & $\mathrm{~T}$ & 1 & $\mathrm{NT}$ & $T$ & T & $\mathrm{T}$ & $\mathbf{T}$ & $T$ & $\mathbf{T}$ & $\mathrm{T}^{\prime}$ & T & $\%$ \\
\hline Pissive: 'Jime-Varying & $\mathrm{NT}$ & $\mathbf{T}$ & $\tau^{\prime}$ & $\tau$ & $\mathrm{I}$ & $\mathrm{NT}$ & $\mathrm{T}$ & $T$ & $\mathrm{~T}$ & $\mathrm{~T}$ & $T$ & NT & $\mathrm{J}$ \\
\hline Heavy Gis: Ground Level & $\mathrm{T}^{*}$ & $\mathbf{T}$ & NT & $\mathrm{NT}$ & $T$ & $\mathrm{~T}$ & $\mathrm{~T}$ & NT & NT & NT & $\mathrm{T}$ & $T$ & $T$ \\
\hline Heavy Gas: Elevated & $\mathrm{T}$ & NT & NT & NT & $T$ & $\mathbf{T}$ & $T$ & NT & NT & NT & $T$ & ]$^{*}$ & $T$ \\
\hline Heuvy Gas: Time-Varying & $N^{\prime} \Gamma$ & $\mathrm{T}$ & $\mathrm{NT}$ & $\mathrm{NT}^{\top}$ & $T$ & Nif & $T$ & NT & NT & $\mathrm{NT}$ & $\tau$ & $\mathrm{NT}$ & $\mathrm{T}$ \\
\hline Liquid Jel Relealse & 3 & NT & $\mathrm{NT}$ & N'] & $\mathrm{T}$ & $\mathrm{NT}$ & $\mathrm{T}$ & NT & NT & NT & $\mathrm{T}$ & $T$ & NT \\
\hline \multicolumn{14}{|c|}{ Physics/Chemistry/Thermodynamics } \\
\hline Entrained Aerosol & $\mathrm{T}$ & $\mathrm{T}$ & NT & NT & $\mathrm{T}$ & $\mathrm{T}$ & $T$ & NT & NT & NT & $\mathrm{T}$ & $\mathrm{N} r$ & $\tau$ \\
\hline $\begin{array}{l}\text { Plume Rise (monenlum, } \\
\text { buoyant) }\end{array}$ & NT & NT & $\mathrm{T}$ & NT & $T$ & $\mathrm{~T}$ & $\mathrm{~T}$ & 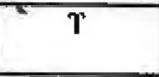 & $T$ & $\mathbf{T}$ & $T$ & $\tau$ & $\mathrm{N}^{\prime} \mathrm{T}$ \\
\hline $\begin{array}{l}\text { Building Wake }>5 \mathrm{Bldg} \mathrm{Hts} \\
\text { downwind }\end{array}$ & NT' & NT & $\mathrm{T}$ & NT & NT & $T$ & $T$ & NT & NT & NT & NT & $\mathrm{T}$ & NT \\
\hline Plume Lift-olt" & NT & $\overline{N r}$ & NT & NT & NT & NT & $\tau$ & NT & NT & NT & NT & NT & $\mathrm{NT}$ \\
\hline Mesoscale Transporl' & $\mathrm{NT}$ & NT & $\mathrm{T}$ & $\mathrm{NT}$ & NT & NT & $\mathrm{N}^{\prime} \mathrm{T}$ & $T$ & NT & $T$ & NT & $\mathrm{NT}$ & $\mathrm{NT}$ \\
\hline Complex Terrain ${ }^{\mathrm{I}}$ & NT & $\mathrm{NT}$ & $\mathrm{T}$ & NT & NT & $T$ & $\mathrm{NT}$ & $T$ & NT & $T$ & NT & $\mathrm{NT}$ & NT \\
\hline Land/Water linterface ${ }^{f}$ & $\mathrm{NT}$ & NT & $\tau$ & NT & NT & NT & $\mathrm{NT}$ & $T$ & NT & NT & NT & NT & NT \\
\hline In-Cloud Chemistry & $\begin{array}{c}T^{3} \\
\left(11 \mathrm{~N} ; \mathrm{N}_{4} \mathrm{O}_{4}\right) \\
\end{array}$ & NT & $\begin{array}{c}\mathrm{T} \\
\left(\mathrm{SO}_{x} \mathrm{NO}_{x}\right)\end{array}$ & NT & $\mathrm{N} T^{\top}$ & NT & $\begin{array}{c}\mathrm{T} \\
\text { (IIFYUP, }\end{array}$ & NT & NT & NT & $\mathrm{NT}$ & $\mathrm{N}^{\prime} \mathrm{T}$ & NT \\
\hline Dry Deposition & $\mathrm{NT}$ & NT & $\mathrm{T}$ & $\mathrm{NT}$ & NT & $\mathrm{T}$ & $\mathbf{T}$ & NT & $\mathrm{T}$ & $T$ & $\mathrm{NT}$ & $\mathrm{N}^{\prime}$ & $\mathrm{T}$ \\
\hline Wet Deposition & NT & NT & $\mathrm{T}$ & NT & NT & NT & $T$ & NT & NT & NT & NT & NT & $\mathrm{T}$ \\
\hline \multicolumn{14}{|l|}{ Oulpu feature } \\
\hline HFward Zone/Fooiprint & $r$ & $T$ & NT & $T$ & $\mathrm{~T}$ & $\Upsilon$ & $\mathrm{T}$ & $T$ & $\mathrm{~N}$ & $T$ & NT & $\mathrm{N} \mathrm{N}^{\prime}$ & $\mathrm{T}$ \\
\hline $\begin{array}{l}\text { Near-Ficld, within I00 in of } \\
\text { the Release (On-Site) }\end{array}$ & $\mathrm{T}$ & $T$ & $T$ & $\mathrm{~T}$ & $\mathrm{~T}$ & $\mathrm{~T}$ & $T$ & NT & $\mathrm{T}$ & NT & $\mathrm{T}$ & $\mathrm{T}$ & $\mathrm{T}$ \\
\hline $\begin{array}{l}\text { Variable Averaging } \\
\text { Time }(<l \text { h) }\end{array}$ & $\mathrm{T}^{\prime}$ & $\begin{array}{c}\mathrm{Nr} \\
(1-\mathrm{hr})\end{array}$ & $\begin{array}{c}\mathrm{NT}^{\mathrm{hi}} \\
(=\mathrm{I} \mathrm{hr})\end{array}$ & $\mathrm{T}$ & $\mathbf{T}$ & $\mathrm{T}$ & $T$ & $\mathrm{~T}$ & $T$ & $\mathrm{~T}$ & $\mathrm{~T}$ & $T^{1}$ & $\mathrm{~T}$ \\
\hline
\end{tabular}


TABLE 5.2 (Cont)

\begin{tabular}{|c|c|c|c|c|c|c|c|c|c|c|c|c|c|}
\hline \multirow[b]{2}{*}{ Model Allribult: } & \multicolumn{13}{|c|}{ Moduls with and without a Particular Capability or Fenture ( $T=$ trealed. NT = not trealed) } \\
\hline & ADAM & ALOHA* & CALPUFF & $\begin{array}{c}\text { CASRAM- } \\
\text { SC } \\
\end{array}$ & DEGADIS" & FEM3C & $\begin{array}{c}\text { HG- } \\
\text { SYSTEM" }\end{array}$ & $\begin{array}{l}\text { HOTMAC } \\
\text { RAPTAD }\end{array}$ & INPUFF & SCIPUFF & SLAB" & TSCREEN & $\begin{array}{l}\text { YLS- } \\
\text { TRAC }\end{array}$ \\
\hline \multicolumn{14}{|l|}{ Ouput Heature (Cour. $)$} \\
\hline Plume Meanderj & $\Gamma$ & NT & $\mathrm{T}^{\prime}$ & $\mathrm{NT}$ & NT & $\mathrm{NT}$ & NT & $\mathbf{T}$ & $\mathrm{NT}$ & $T$ & $T$ & $\mathrm{NT}$ & T \\
\hline 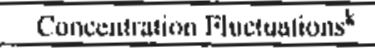 & NT' & NT & $\mathrm{N}]^{\top}$ & $\mathrm{NTl}$ & $\mathrm{NT}$ & $\mathrm{T}$ & $\mathrm{T}$ & $T$ & NT & $\mathrm{T}$ & $\mathrm{T}$ & $\left.N^{\prime}\right]^{\prime}$ & Ne \\
\hline $\begin{array}{l}\text { Concelotrution, Jiute } \\
\text { 3isistoriest }\end{array}$ & "l" & $T$ & NT & $\mathrm{N}^{\prime} \mathrm{N}^{\prime}$ & $\mathrm{T}$ & $\mathrm{J}^{4}$ & $T$ & $T$ & $T$ & $T$ & $\mathrm{~T}$ & $\mathrm{NT}$ & $\mathrm{T}$ \\
\hline $\begin{array}{l}\text { Tunde-Intetraled } \\
\text { Concentraltons" }\end{array}$ & $\mathrm{T}$ & $\mathrm{T}$ & $\mathrm{Nr}$ & $\mathrm{Ni}$ & $\mathrm{NT}$ & $\pi$ & $\mathrm{T}$ & $\mathrm{T}$ & $T$ & $T$ & $\mathrm{NT}$ & $\overline{N T}$ & $T$ \\
\hline Uneertainly Analysis & $N T$ & NT & $\mathrm{Ni}$ & $\mathrm{T}$ & $\mathrm{NT}$ & $\mathrm{NT}$ & $\mathrm{NT}$ & NT & $\mathrm{NT}$ & $\mathbf{T}$ & NT & $\mathrm{NI}$ & $\mathrm{N1}$ \\
\hline Propnostic & NT & NT & $T$ & $\mathrm{NT}$ & $\mathrm{NT}$ & NT & NT & $T$ & NT & NT & NT & $N T$ & $\mathrm{NI}^{\prime}$ \\
\hline Risk Asstssmient & $\mathrm{NT}$ & NT & NT & $\Gamma$ & $\mathrm{NT}$ & NT & $\mathrm{NT}$ & $\mathrm{NT}$ & NT & NT & $\mathrm{NT}$ & $\mathrm{NT}$ & $\mathrm{N}^{\prime \prime} \mathrm{T}^{*}$ \\
\hline $\begin{array}{l}\text { Performance Evalwation" } \\
(\mathrm{Y}=\mathrm{Yes} / \mathrm{N}=\mathrm{Nu})\end{array}$ & & & & & & & & $=$ & & & & & \\
\hline $\begin{array}{l}\text { Developer/Lndependent } \\
\text { Field/Luboritory Dita }\end{array}$ & $\mathrm{Y} / \mathrm{Y}$ & $N^{m} N$ & $\mathrm{Y} / \mathrm{Y}$ & $N^{*} / N$ & $\bar{Y} / \mathrm{Y}$ & $\bar{Y} / \mathrm{Y}$ & $\overline{Y N}$ & $\mathrm{YN}$ & $\bar{Y} / \mathrm{Y}$ & $Y N$ & $Y \sim$ & NN & $Y \mathbb{N}$ \\
\hline
\end{tabular}

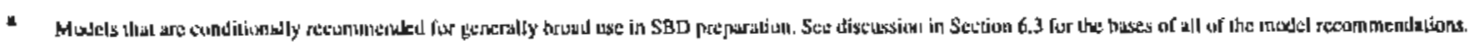

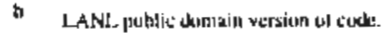

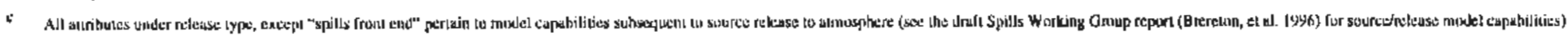

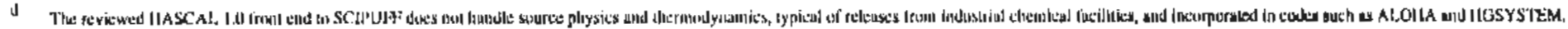

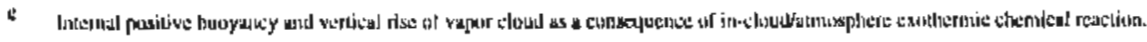

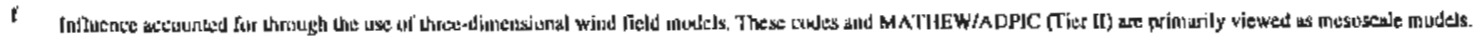

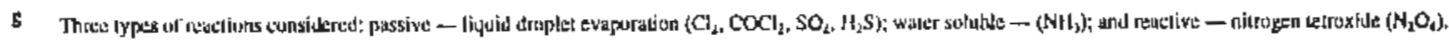

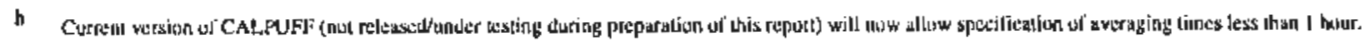

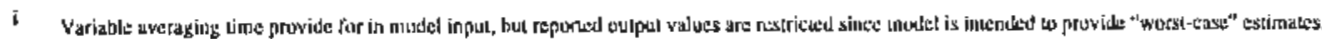

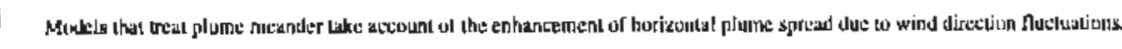

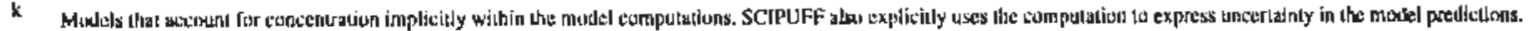

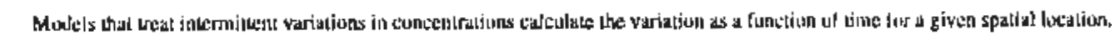

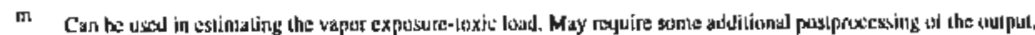

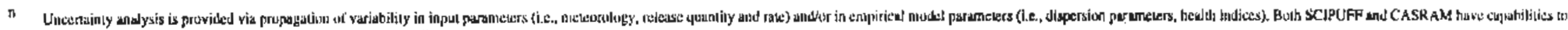

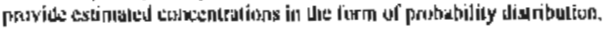

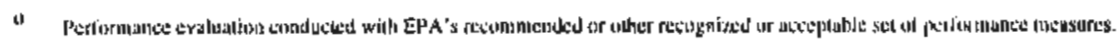

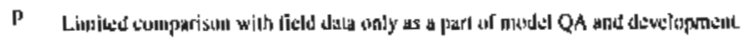


Four major categories of characteristics are included in Table 5.2 - release type, physics/chemistry/thermodynamics, output features, and performance evaluation. An extensive set of footnotes is included to clarify the contents of this tabular summary. Each of the four categories is discussed below.

\section{Release Type}

Spills Front End - Although not a release category, the first attribute (spills front end) is inclnded to indicate if the code has the capability to compute a time-dependent liquid, gas, and/or two-phased release vapor. The model would be capable of handling a variety of accidental release conditions (e.g. horizontal jet) and the dispersion of the resulting vapor cloud. 14

Each of the seven test problems fell into one (or more) of the next seven vapor release categories designed to encompass most of the more probable DOE release scenarios. Note that use of the word "passive" primarily was meant to distinguish neutral and buoyant from "heavy-gas" releases. Some codes also consider chemical reactions for which there is no distinction in the seven categories.

Passive: Ground Level - A release at ground level of a neutrally buoyant plume.

Passive: Elevated - A release above the ground (e.g., from a stack) of a neutrally buoyant vapor. Plume rise can occur due to momentum. For this study, buoyant releases were also included in this category; however, strictly speaking they are not "passive" releases.

Passive: Time-Varying - A longer-term release of a neutrally buoyant vapor that changes magnizude every 15 minutes or less.

Heavy Gas: Ground Level - A release at ground level of a heavier-than-air vapor. The vapor could be heavy because of its molecular weight being higher than that of air and/or because of an increase in density at lower temperatures.

Heavy Gas: Elevated - A release above the ground of a heavier-than-air vapor that can sink after release because of its negative buoyancy.

Heavy Gas: Time-Varying - A longer-term release of a heavier-than-air vapor that changes magnitude every 15 minutes or less.

14 Although Table 5.2 indicates that some models (i.e., DEGADIS, HGSYSTEM, SLAB) treat all or most of the release types listed, this may not be the case for all variations of that release type. For example, the DEGADIS (Ver. 2:1) handles vertical but not horizontal jets. 
Liquid Jet Release - A release of a liquid chemical with a significant exit velocity. Modeling of jet effects is often important in determining initial dispersion characteristics.

\section{Physics/Chemistry/Thermodynamics}

Entrained Aerosol - Liquid droplets that are mixed in the plume initially after release. During the release of pressurized liquids, some amount of the liquid may not vaporize and will be entrained within the gaseous plume as aerosols (droplets). Models that incorporate the proper physics for deposition and entrainment fall into this category.

Plume Rise (momentum, buoyant) - Plume rise is computed above the release point because of heated and/or mechanically exhausted effluent.

Building Wake $>5$ building heights downwind - The initial dispersion of a plume release in or near the aerodynamic building wake. For this evaluation, these effects are considered beyond five building heights downwind from the release point.

Plume Lift-Off - Plume rise of vapor cloud above the release elevation because of internal positive buoyancy and/or as a consequence of in-cloud/atmosphere exothermic chemical reaction.

Mesoscale Transport - Vapor cloud transport well beyond the release point into flow regimes that are influenced by factors other than the meteorological conditions at the release location. This influence is often accounted for through the use of threedimensional wind field models.

Complex Terain - Perturbations in the wind field that affect plume transport and dispersion caused by terrain features. These effects are often accounted for through the use of three-dimensional wind field models and particle-in-cell or puff dispersion models.

Land/Water Interface - Perturbations in the wind field that affect plume transport and dispersion primarily caused by the difference in temperature (and surfaces) that often exist between land and water. Atmospheric conditions near shorelines are not aiways represented accurately by data measured from a single location. These effects are often accounted for through the use of thermal internal boundary layer (TIBL) models or three-dimensional wind field models.

In-Cloud Chemistry - Three types of in-cloud chemistry are considered: (1) passive (liquid droplet evaporation), (2) water soluble (chemicals change characteristics in the presence of water), and (3) reactive (chemical reactions take place in the cloud).

Dry Deposition - Removal of particulate from the plume by deposition on the ground surface due to gravity and other factors such that they are no longer considered in the cloud concentration. 
Wet Deposition - Removal of particulates from the plume by capture in precipitation and deposition on the ground surface such that they are no longer considered in the cloud concentration.

\section{Output Features}

Hazard Zone/Footprint - A tabular and/or graphic representation of the area where the concentration exceeds a specific hazard level.

Near-Field, within $100 \mathrm{~m}$ of the Release (On-Site) - Capability of a model to compute concentration within $100 \mathrm{~m}$ of the release location. Computing on-site concentration is complicated by features such as buildings and dikes and release characteristics such as exit velocity, sinking heavy gas, and angle of release.

Variable Averaging Time ( $<1$ hour) - Computation of the average concentration for a time period less than one hour and selectable by the user.

Plume Meander - A tendency for a plume under low wind speeds (less than $6 \mathrm{~m} / \mathrm{s}$ ) in stable atmospheric conditions to change direction in the horizontal plane such that the plume is wider than would be predicted by use of, for example, the standard deviation of wind direction $(\sigma y)$ for a PG stability class. The correction is made in different ways, including changes in wind direction and changes to $\sigma y$ as a function of averaging time.

Concentration Fluctuations - A capability to account for concentration variations imphicitly within the code computations.

Concentration, Time Histories - A capability to treat intermittent variations in concentrations as a function of time for a given spatial location.

Time-Integrated Concentrations - Accumulation of vapor concentration over a period of time for use in estimating the vapor exposure-toxic load. May require some additional postprocessing of the output.

Uncertainty Analysis - A propagation of variability in input parameters (e.g., meteorology, release quantity and rate) and/or in empirical model parameters (e.g., dispersion parameters, health indices).

Prognostic - A capability to forecast future weather patterns controlling plume transport and dispersion on the basis of current measurements of meteorological conditions.

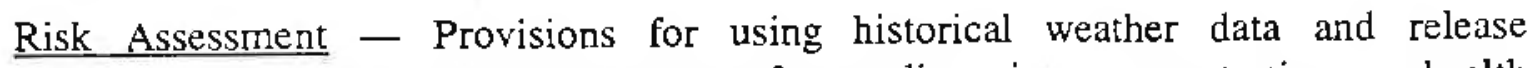
probabilities to determine the frequency of exceeding given concentrations or health effects. 


\section{Performance Evaluations}

By Developer/Independent Reviewer Using Field or Laboratory Data - An indication of the extent of comparison of a given code with measured data from laboratory or field tests.

\subsection{TEST PROBLEM FINDINGS}

Table 5.3 summarizes the test cases, described in Chapter 4 , that were run for each of the Tier I models. Results from 3 (CASRAM-SC, HOTMAC/RAPTAD, and SCIPUFF) of the 13 Tier I models reviewed are not reported because of the lack of a user guide and/or full or complete technical documentation, and/or their beta-version state at the time of our review. With the exception of CASRAM, these models have been published in the peer-reviewed literature and are well known in the air dispersion modeling community. SCIPUFF and HOTMAC/RAPTAD (proprietary version) are established, widely used models whose latest versions are still in the process of being tested and/or further developed.

\subsubsection{Analysis of Results of Test Cases}

The model predictions for each of the test cases are plotted in Figures 5.1 through 5.21 (which are at the end of the chapter). Curves for all models are plotted together so that they can be visually compared. It should be pointed out that some models are missing from some plots, because not all models could be successfully applied to all scenarios (in some cases, the model would not run the test case, in other cases the model was not applicable to the particular test case). Some models appear to be outliers on the plots. The reasons for that situation, if known, are discussed below.

TSCREEN often predicts high. This is intentional; in fact, the model automatically runs through a series of stability class/wind speed combinations in order to choose a "worst case." Thus, although the test case may be defined as neutrally stable atmospheric conditions (Class D) with $5 \mathrm{~m} / \mathrm{s}$ winds, the TSCREEN model may choose stable conditions (Class $F$ ) with $2 \mathrm{~m} / \mathrm{s}$ winds. For this reason the TSCREEN predictions should not be used in defining ranges of model predictions except for scenarios using the same conditions that TSCREEN selected as the "worst case." Because of the design of TSCREEN, its predictions depicted in Figures 5.1 through 5.21 should be conservative (i.e., worst-case predictions) compared with the other models.

VLSTRACK often produces predictions whose magnitude and slope (with distance) disagree with the other models. Since VLSTRACK is a Department of Defense model intended for application to specific munitions, it has not been tested for a wide variety of release scenarios. Thus, we recommend that VLSTRACK predictions not be included in comparing the ranges across all the models.

Most of the test cases involve plumes with initial densities greater than that of air. Two (CALPUFF and INPUFF) of the 10 Tier I model comparison results reported here do not treat dense gases. Consequently, the predictions of these models are not expected to be accurate 
TABLE 5.3 Test Cases for Tier I Model Accident Scenario Applications

\begin{tabular}{|c|c|c|c|c|c|c|c|c|}
\hline \multicolumn{10}{|c|}{ Tier I Accidentai Release Scenario Simulations } \\
\hline
\end{tabular}

2 Model does not trest dense-gas effects

close to the source in cases when dense-gas effects are important. The plotted modeling results for CALPUFF and INPUFF are intended to illustrate how these models compared with codes designed to handle dense-gas releases.

Within the context of the above caveats, results of the test cases are discussed below. No quantitative comparisons, such as biases or mean-square errors, have been calculated because these test scenarios are only hypothetical cases, and there is no "right" answer. Instead, the discussions center on the range of the predictions by the various models at certain key points.

\subsubsection{Discussions of Model Comparisons for Each Test Case}

Test Case A - Time-Variable Liquid Chlorine Spill at Ambient Temperature (Figures 5.1a-f through 5.3): Plots are given for several averaging times. Because it can be confusing to attempt to make comparisons from one averaging time to another, and because some models (NNPUFF, HGSYSTEM, and SLAB) correct for averaging time and other models (ADAM, DEGADIS, and ALOHA) do not, these discussions center on the results for a 10 minute averaging time (Figure 5.1a). As found by Hanna et al. (1993) for several field tests, DEGADIS is seen to predict relatively high near the source. At large distances downwind $(\sim 10 \mathrm{~km})$ INPUFF predicts at the low end of the range, and HGSYSTEM predicts at the high end. The model curves are not bunched together on the figure, but seem to cover a range of less than an order of magnitude. When we look at a distance of $1,000 \mathrm{~m}$, the predictions cover a range of a factor of about 14 (i.e., 75 ppm for ADAM to 1,343 ppm for HGSYSTEM). ADAM tends to have a negative bias (lower estimates) compared with the other models in the downwind range from $50 \mathrm{~m}$ to $1.5 \mathrm{~km}$.

The test case A-1 variant, with stability/wind speed changed from $F / 2$ to $D / 5$ (Figure 5.2), shows better agreement in model predictions. This result is seen consistently across all test cases - the model predictions agree best for neutral conditions with moderate wind speeds. At a distance of $1,000 \mathrm{~m}$, the predictions of concentration agree within a factor of 3 . At a distance of $100 \mathrm{~m}$, the predicted concentrations also agree within a factor of 3 . ADAM tends to 
have a negative bias (lower estimates) compared with SLAB and HGSYSTEM in the downwind range from 100 to $1,000 \mathrm{~m}$, but not as large as the A-0 cases. This observation is consistent with ADAM's observed negative bias tendency when its predictions, along with the predictions of 13 other models, are compared with the Desert Tortoise $\left(\mathrm{NH}_{3}\right)$ field observations (Hanna 1991). 15

The factor of 10 increase in surface roughness evaluated with the test case A-2 variant shows that the reduction in magnitude of ADAM's predictions compared with those of SLAB and HGSYSTEM are larger close in $(\leq 500 \mathrm{~m})$ and smaller farther out $(>1,000 \mathrm{~m})$. The magnitude of the disagreement between models is about the same as in test case A-0. HGSYSTEM and SLAB are in good agreement except near the source $(<100 \mathrm{~m}$ downwind).

Test Case B - Refrigerated Liquid Chlorine Spill (Figures 5.4 through 5.6): Test case B is similar to case A, except for case B the chlorine is refrigerated and the emission rate is constant. Although curves are given for 10 models in Figure 5.4a and 6 models in Figure 5.4b for the reasons discussed in Section 5.2.1, a lower weight should be given to VLSTRACK, and TSCREEN should be viewed as a worst case (F/1). ADAM is consistently low, as it is for most of the test cases. The EPA's INPUFF and CALPUFF models generally agree with the dense-gas models, since the plume density is close to neutral at downwind distances beyond 50 to $100 \mathrm{~m}$. FEM3C predicts near-freld concentration values exceeding those of all the other codes evaluated for test case B-0 in the range of 50 to $100 \mathrm{~m}$. Between 100 and $200 \mathrm{~m}$, the FEM $3 \mathrm{C}$ results fall within the upper and lower bounds on the results obtained from the other models. Neglecting ADAM (which is an outlier on this plot), the range in model predictions of concentration at a downwind distance of $1,000 \mathrm{~m}$ is about a factor of 10 . At a distance of $100 \mathrm{~m}$, neglecting ADAM for the same reason and VLSTRACK (which may not handle density effects that would be evident here), the downwind concentrations differ by about a factor of 3 .

The test case B-1 variant, with stability class/wind speed changed from $F / 2$ to $D / 10$ (Figure 5.5a), shows a tendency for model curves to converge, as was found for test case A. TSCREEN is an outlier because it is still using F/1 to calculate a worst case. Ignoring VLSTRACK, the predictions agree within a factor of 2 , since all models asymptotically approach a standard Gaussian plume model, with standard EPA sigmas in the limit of neutral stability with high winds.

The test case B-2 variant (Figures 5.6a and 5.6b) increases the receptor height from 0 to $1 \mathrm{~m}$. The results exhibit little change from the B-0 case. Only DEGADIS, HGSYSTEM, and SLAB appear to have lower concentrations. The crosswind widths vary widely between models, as shown in Figures 5.5b and 5.6b.

Test Case C - Liquid Acetone Spill and Evaporation (Figures 5.7 through 5.9): Acetone does not evaporate as rapidly as chlorine. Consequently, test case $\mathrm{C}-0$ (Figure 5.7) reduces to an area source of nearly neutrally buoyant gas. Furthermore, the base case is associated with neutral conditions and a wind speed of $5 \mathrm{~m} / \mathrm{s}$. As expected, the model predictions

15 ADAM tends to underpredict observed concentrations at 100 and $800 \mathrm{~m}$ by a factor of two or three. 
in Figure 5.7 are similar (discounting TSCREEN, which chooses an F/l as the worst case). The curves for five models (HGSYSTEM, SLAB, CALPUFF, ALOHA, and INPUFF) fall nearly on top of each other, with disagreements of about a factor of 2 .

The test case C-1 variant, with stability class/wind speed changed from $D / 5$ to $F / 2$ (Figure 5.8), still shows good agreement among most models, but the scatter of the curves now increases to about a factor of 2 everywhere. The slippage of model conformity for stable conditions is found in most test case results.

In the test case $\mathrm{C}-2$ variant, the area of the source increases by a factor of 4 (Figure 5.9). For these conditions, the HGSYSTEM curve is about a factor of 3 or 4 above the other curves. It is difficult to interpret these results. The models may be increasing their calculation of source evaporation rate, and it is not certain how the calculated virtual source distance from the release location is changing as the size of the area source increases.

Test Case D - Vertically Pointing Butane (Heavy Gas) Jet (Figures 5.10 through 5.12): Test case D is included because the dense-gas plume will sink to the ground within $50 \mathrm{~m}$ of the source, even though it is initially directed upwards at great speed. CALPUFF and INPUFF cannot account for dense gases and, therefore, do not allow the dense-gas plume to settle to the ground quickly enough. For case D-0 (Figure 5.10), predictions of the dense-gas models (SLAB, HGSYSTEM, DEGADIS, and ALOHA) are spread over a range of about a factor of 5 to 7 at distances greater than $400 \mathrm{~m}$, with SLAB at the high end of the range and DEGADIS at the low end. The results are about the same for the two variants to the test case (variant D-1 - increased stack height; variant D-2 - increased initial jet speed [Figures 5.11 and 5.12]). With the higher stack height or wind speed, the plumes calculated by CALPUFF and INPUFF stay aloft even longer before impacting the ground. For these types of scenarios (i.e., dense-gas jets released upward from stacks), use of a dense-gas model is essential.

Test Case E-Warm Gas Plume with $\mathrm{H}_{2} \mathrm{SO}_{4}$ Mist from Building Vent (Figures 5.13 through 5.15): Scenario $E$ does not involve dense gases at all. In fact, the plume is initially buoyant and is released from a vent on the roof of a building. For test case E-0 (Figure 5.13), the predictions of the SLAB, CALPUFF, and ALOHA models agree within a factor of about 2 at distances beyond about $500 \mathrm{~m}$. At shorter distances, the CALPUFF model more accurately accounts for the downwash effects of the building; whereas SLAB and ALOHA do not account for the aerodynamic effects of the building. The TSCREEN model is probably picking a different combination of stability class and wind speed, which would account for its different slope. The FEM3C results for the E-0 test case are relatively high in the near-field range of 50 to $100 \mathrm{~m}$ downwind from the stack location. As explained in the detailed discussion of the FEM $3 \mathrm{C}$ sample problem evaluations (Appendix F), an extremely small time step size was required to preserve stability in the evolving flow field. A combination of slip boundary conditions and mixed boundary conditions was applied along the sides of the building, which would allow for zero normal velocities and still permit a large degree of tangential flow. The concentration values in the lee side of the building exhibited oscillations both in time and space, which could be interpreted as either high downflow activity along the sides of the building or insufficient spatial resolution in the wake cavity. FEM3C results appear to be approaching the results of CALPUFF in the far field, but results beyond $500 \mathrm{~m}$ were not reported because CPU time constraints 
required truncation of the computational grid. The downflow phenomenon appears not to be present in the FEM3C E-1 test case results with the F stability conditions. The two test variants (Figures 5.14 and 5.15) involve (1) $\mathrm{F} / 2$ instead of $\mathrm{C} / 2$, and (2) a taller building. The resulting differences in the models as seen on the plots are similar to test case E-O (Figure 5.13).

Test Case F - Dense Gas Jet, at Elevation of I m, Pointing Horizontally Downwind (Figures 5.16 through 5.18): Seven model curves are plotted on Figure 5.16 for test case F $\sim 0$. It should be noted that TSCREEN is picking a worst case and, therefore, should not be considered in defining the model ranges. Five of the models (SLAB, INPUFF, CALPUFF, ALOHA, and DEGADIS) agree remarkably well - within about $50 \%$ at most distances. The HGSYSTEM curve, which is about a factor of 2 below the others at small distances, is more correct because it is accounting for the dynamics of jet entrainment and interactions with the nearby ground surface. The non-dense-gas models (INPUFF and CALPUFF) are doing relatively well because the concentration quickly drops to levels such that dense-gas effects are no longer important. The results for variants $F-1$ and F-2 (Figures 5.17 and 5.18) (which change from $F / 1$ to $D / 5$ and increase in release rate from $5 \mathrm{~kg} / \mathrm{s}$ to $20 \mathrm{~kg} / \mathrm{s}$, respectively) are similar. Note that for the larger release (test case F-2), the predictions of HGSYSTEM at distances greater than $500 \mathrm{~m}$ are even further below (now a factor of 3 or 4) those of the other models than for test case F-0 (Figure 5.16).

Test Case G-Short Duration HF Gas/Aerosol Mixture Released from Area Source (Figures 5.19 through 5.21): This test case is intended to simulate a two-phase pressurized jet. However, for most of the models to handle the scenario, it had to be modified so that it was treated as an area source. Also, the phase changes and thermodynamic effects of the aerosols are not accounted for. As before, TSCREEN and VLSTRACK should not be included in the comparisons of curves on the figures. Note in Figure 5.19a for case G-0 that ADAM now tends to have a slight (at $100 \mathrm{~m}$ ) to a strong (at $1,000 \mathrm{~m}$ ) positive bias compared with HGSYSTEM and SLAB predictions. Also note that HGSYSTEM, SLAB, ALOHA, and DEGADIS appear to agree within a factor of about 4 at $100 \mathrm{~m}$ and within a factor of about 2 at 1,000 m. Those four models should produce the best answers for this scenario hecause they include the proper physical assumptions for dense-gas releases. FEM3C compares somewhat high with HGSYSTEM, SLAB, and DEGADIS considering that the option for phase change and heat transfer was activated in FEM3C for this case, and, therefore, it was expected that the physics of the problem would be modeled similarly to the other models. Even INPUFF produces a reasonable curve, despite the fact that it does not account for dense gases. Wide variation in results is exhibited for crosswind concentrations, as seen in Figures 5.19b through 5.19d.

With variant G-1 (Figure 5.20), where the stability class/wind speed is changed from $\mathrm{F} / 2$ to $\mathrm{D} / 8$, the predicted concentrations all drop by about an order of magnitude, and the plume acts more like a nonbuoyant release at downwind distances beyond $50 \mathrm{~m}$. ADAM predictions are still a factor of about 5 greater than predictions of the other models at distances beyond about $500 \mathrm{~m}$.

With variant G-2 (Figure 5.21), involving an increase in the duration of the release from 1 minute to 5 minutes, the chrves converge. This release is more like a continuous plume, which the models are expected to better simulate than shorter finite-duration releases. FEM3C predicts 
relatively little dispersion of the cloud between 50 and $100 \mathrm{~m}$. This follows, in part, because zero pressure boundary conditions were imposed on a subdomain of the spatial grid to model the blocking effects of such a large quiescent aerosol cloud released over a relatively short time period. However, upon review of the results, it may be that the blocking effects were overstated in the problem setup. This situation illustrates the fact that a code with more flexibility in problem description can exhibit a wide variety of results, which need to be correlated to actual experiment. Once the vapor escapes the blocked domain (where velocities and pressure gradients were set to zero), the cloud rapidly disperses.

Table 5.4 presents the results of two simplified summaries of the data where attempts were made to identify trends. The results of the four conditionally recommended codes (see discussion in Section 6.3 for the basis of the model recommendations) were computed at distances of 100 and $1,000 \mathrm{~m}$ downwind. In the two left-hand columns, the average spread or difference in predicted concentrations between one code and another are compared for each test run and are averaged over all test runs for distances of 100 and $1,000 \mathrm{~m}$, respectively. The results indicate that no single code is always at yariance with the others since the average spread is always between about 3.0 and 4.0 at $100 \mathrm{~m}$ and between 2.4 and 3.5 at $1,000 \mathrm{~m}$ with little difference between models.

In the second comparison (the two right-hand columns) an attempt was made to find the relative bias among codes on the high side or low side. Each code for each test was indexed from 1 to 4 (highest to lowest) on the basis of its concentration predictions. As shown, the average relative bias indexes for the codes appear to be very different, indicating that no one code is always producing higher or lower results than another.

TABLE 5.4 Comparison of Results for Conditionally Recommended Codes

\begin{tabular}{|c|c|c|c|c|c|}
\hline \multirow{2}{*}{\multicolumn{2}{|c|}{ Code }} & \multicolumn{2}{|c|}{$\begin{array}{l}\text { Average High/Low } \\
\text { Spread Ratio }\end{array}$} & \multicolumn{2}{|c|}{$\begin{array}{l}\text { Average High/Low } \\
\text { Bias Index }\end{array}$} \\
\hline & & @ $100 \mathrm{~m}$ & (@) $1,000 \mathrm{~m}$ & (a) $100 \mathrm{~m}$ & (@) $1,000 \mathrm{~m}$ \\
\hline \multicolumn{2}{|c|}{ ALOHA } & 3.5 & 2.9 & 2.0 & 2.3 \\
\hline \multicolumn{2}{|c|}{ DEGADIS } & 4.0 & 3.5 & 1.8 & 2.5 \\
\hline \multicolumn{2}{|c|}{ SLAB } & 3.0 & 2.9 & 2.2 & 1.4 \\
\hline \multicolumn{2}{|c|}{ HGSYSTEM } & 3.9 & 2.4 & 2.4 & 2.0 \\
\hline \multicolumn{6}{|c|}{ 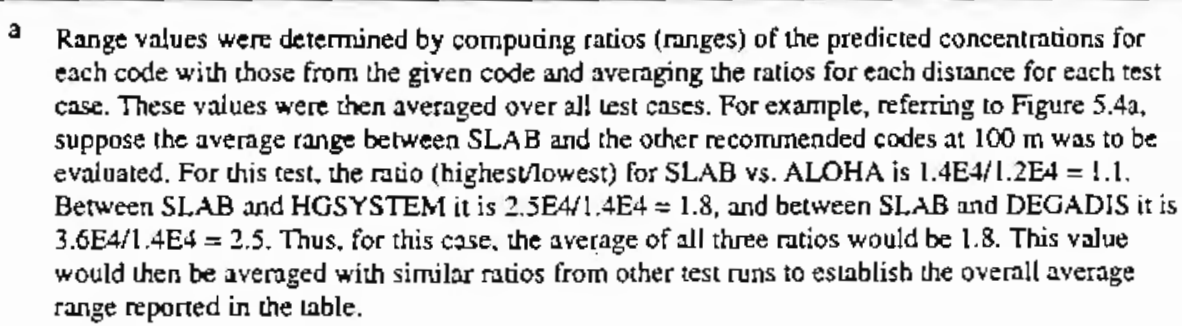 } \\
\hline \multicolumn{6}{|c|}{$\begin{array}{l}\text { The highllow bias index was determined by ordering the codes from } 1 \text { to thighest to lowest) on } \\
\text { the basis of predicted concentrations at each distance for each test run and averaging the results. }\end{array}$} \\
\hline
\end{tabular}




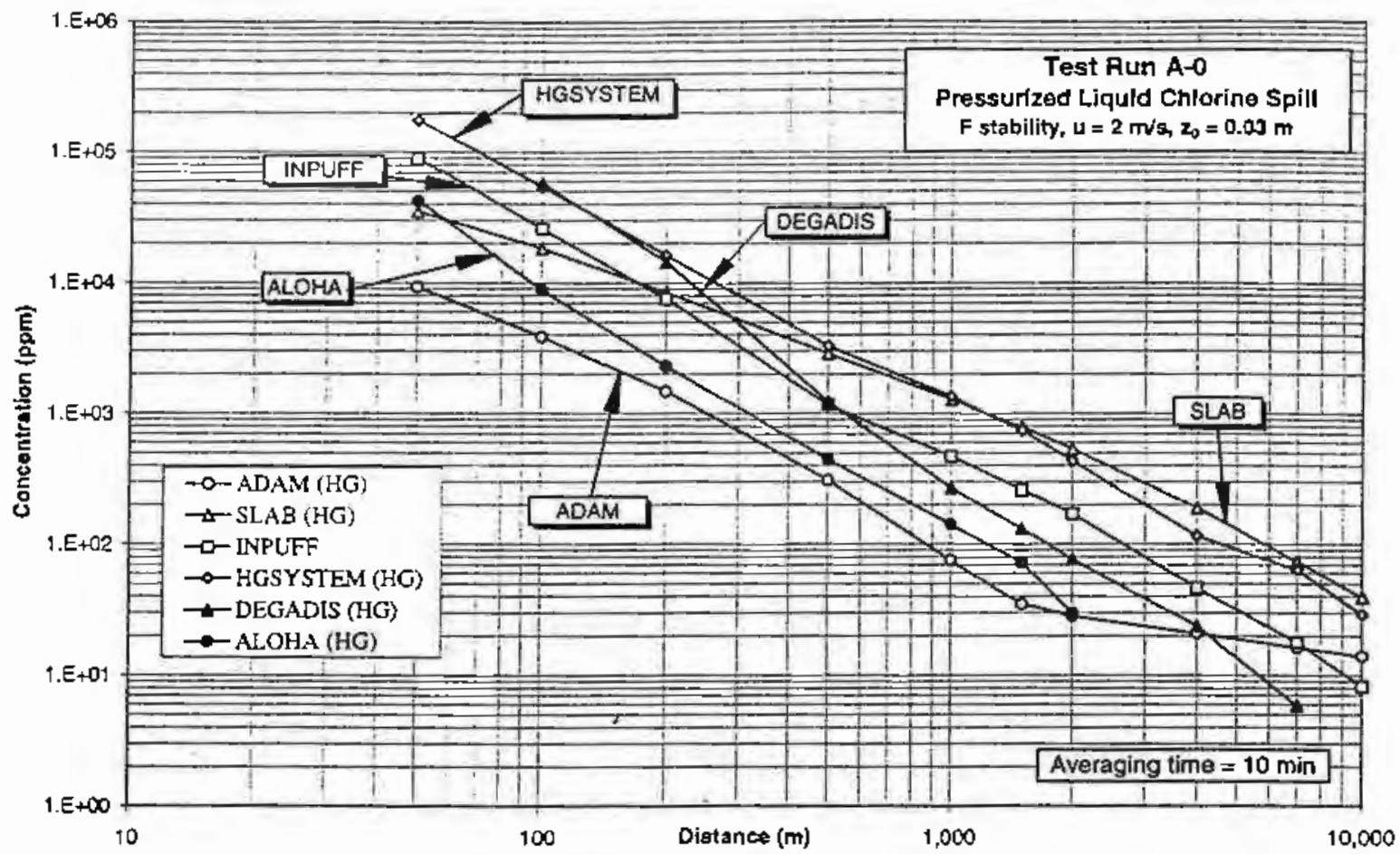

FIGURE 5.1a Base Case: Predicted Ground-Level Centerline Concentration vs.

Downwind Distance for Transient Pool Evaporation - 10 Minute Average

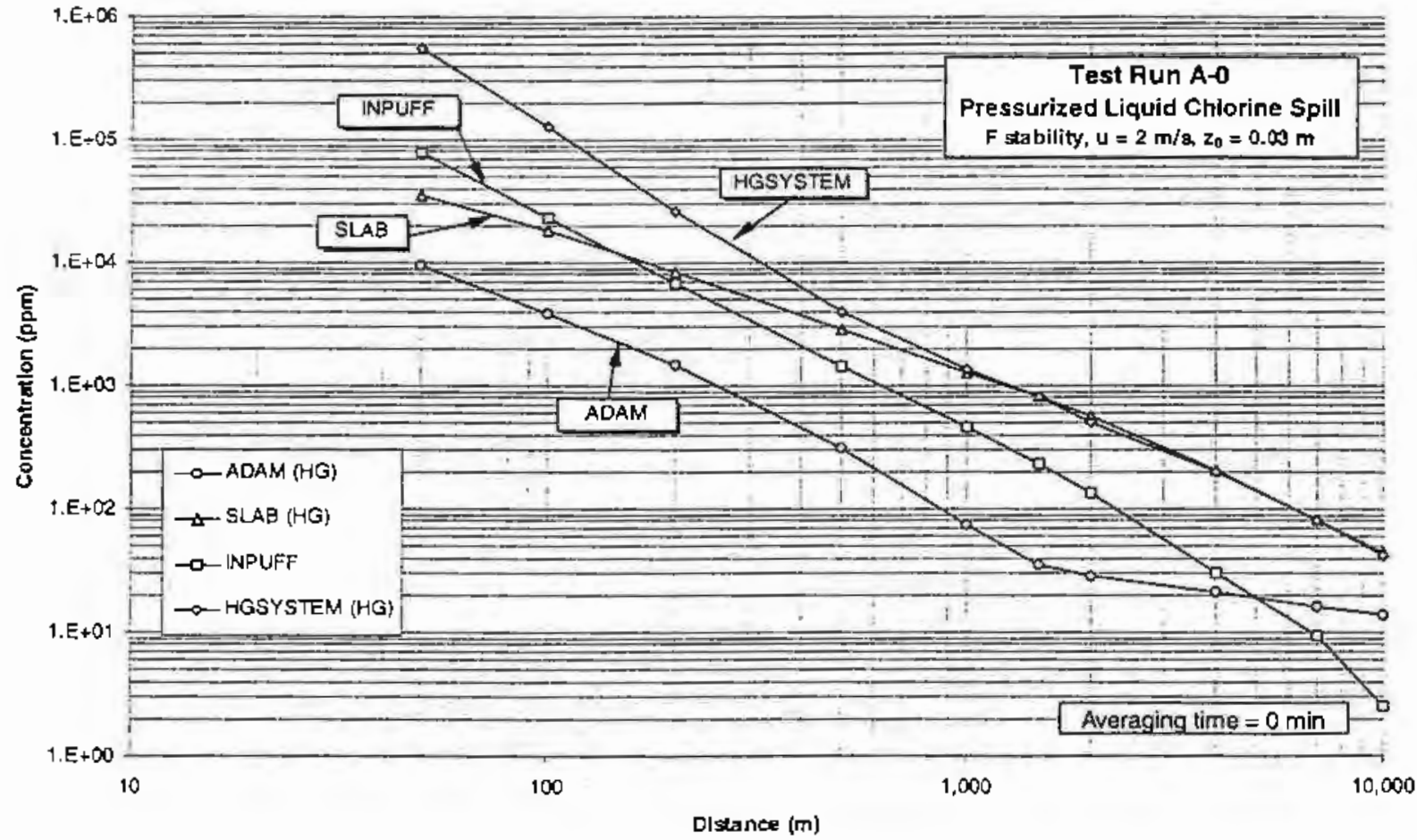

FIGURE 5.1b Base Case: Predicted Ground-Level Centerline Concentration ys. Downwind Distance for Transient Pool Evaporation - Instantaneous Concentration 


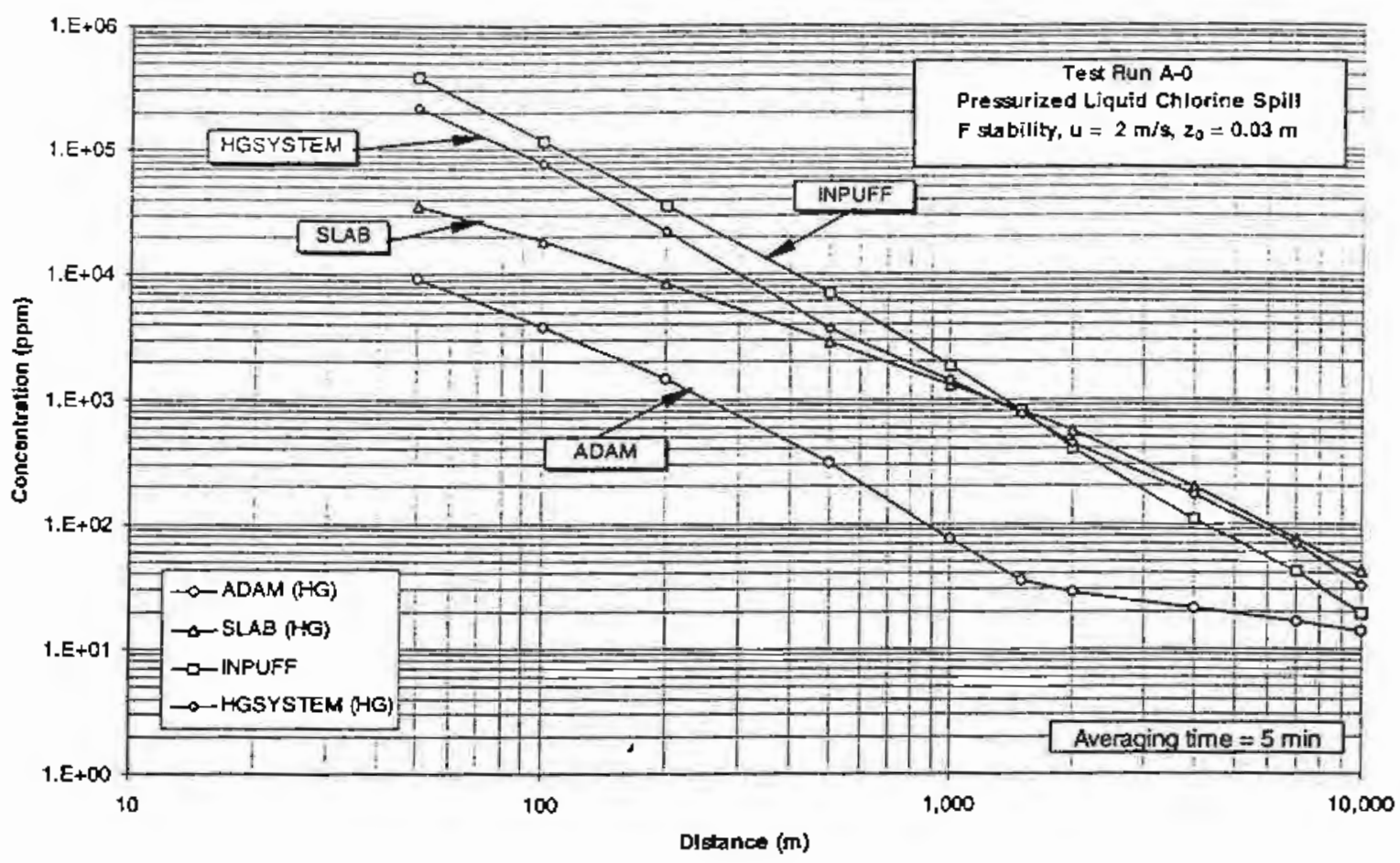

FIGURE 5.1c Base Case: Predicted Ground-Level Centerline Concentration vs. Downwind Distance for Transient Pool Evaporation - 5 Minute Average

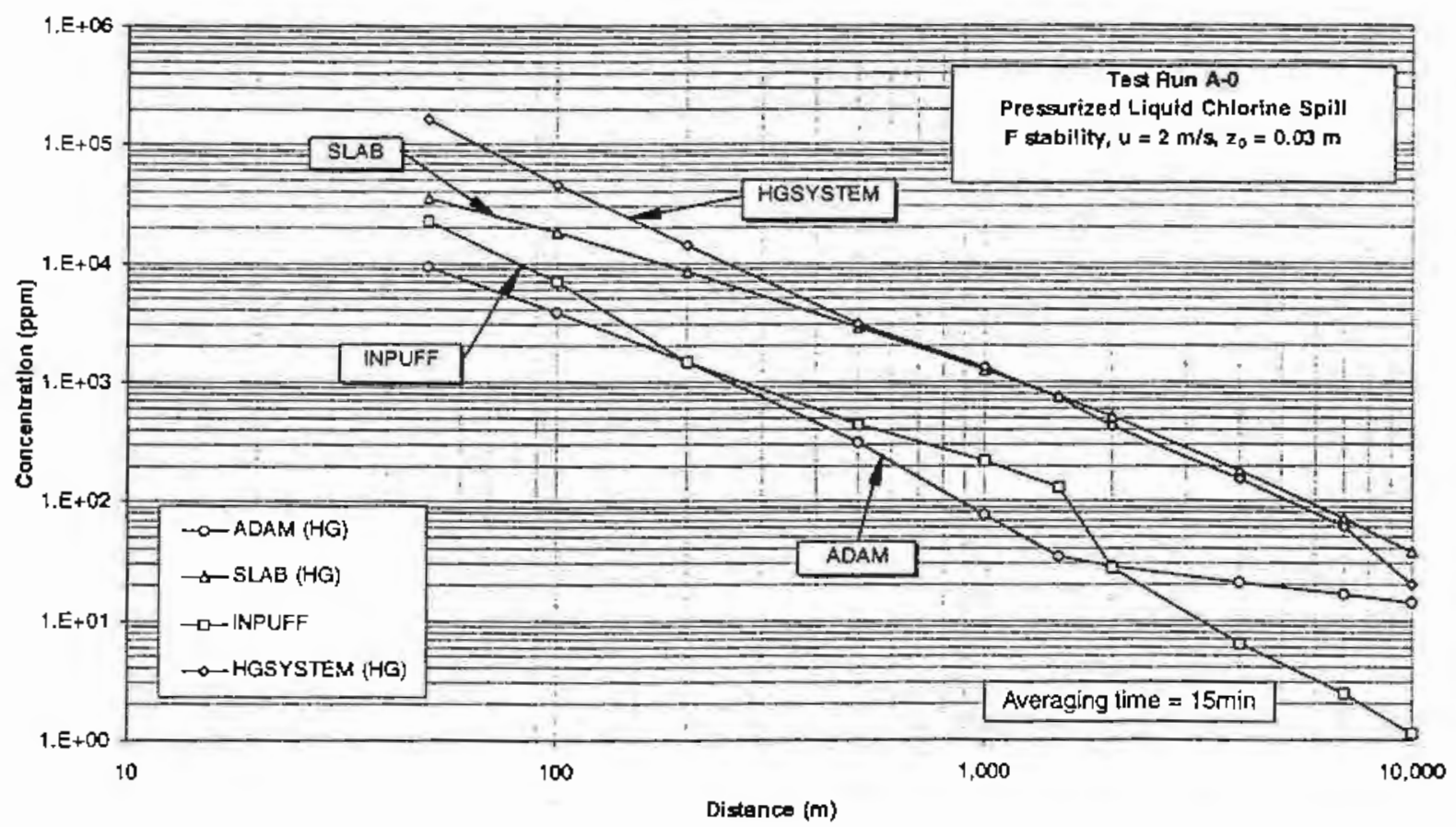

FIGURE 5.1d Base Case: Predicted Ground-Level Centerline Concentration vs. Downwind Distance for Transient Pool Evaporation - 15 Minute Average 


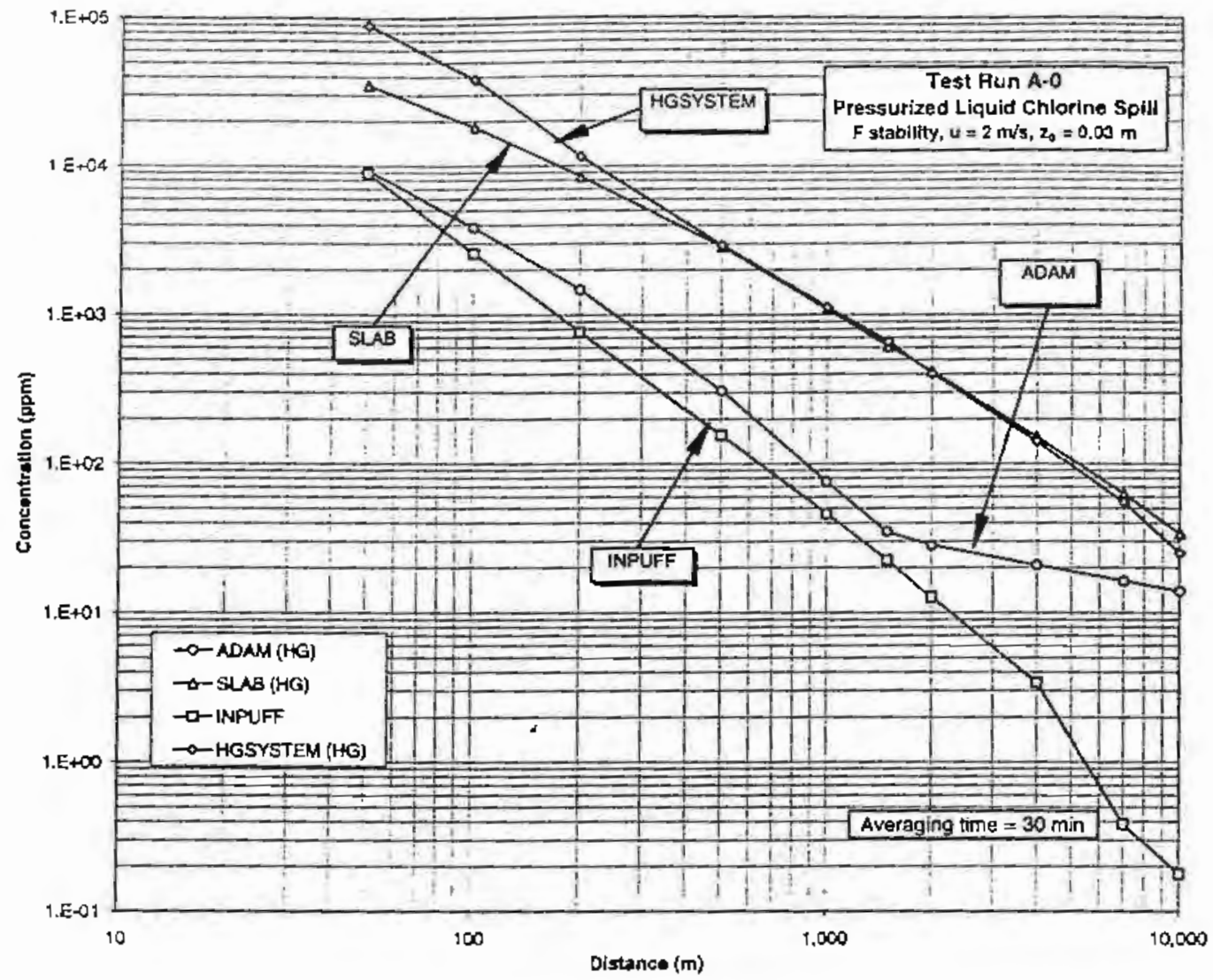

FIGURE 5.1e Base Case: Predicted Ground-Level Centerline Concentration vs. Downwind Distance for Transient Pool Evaporation - 30 Minute Average

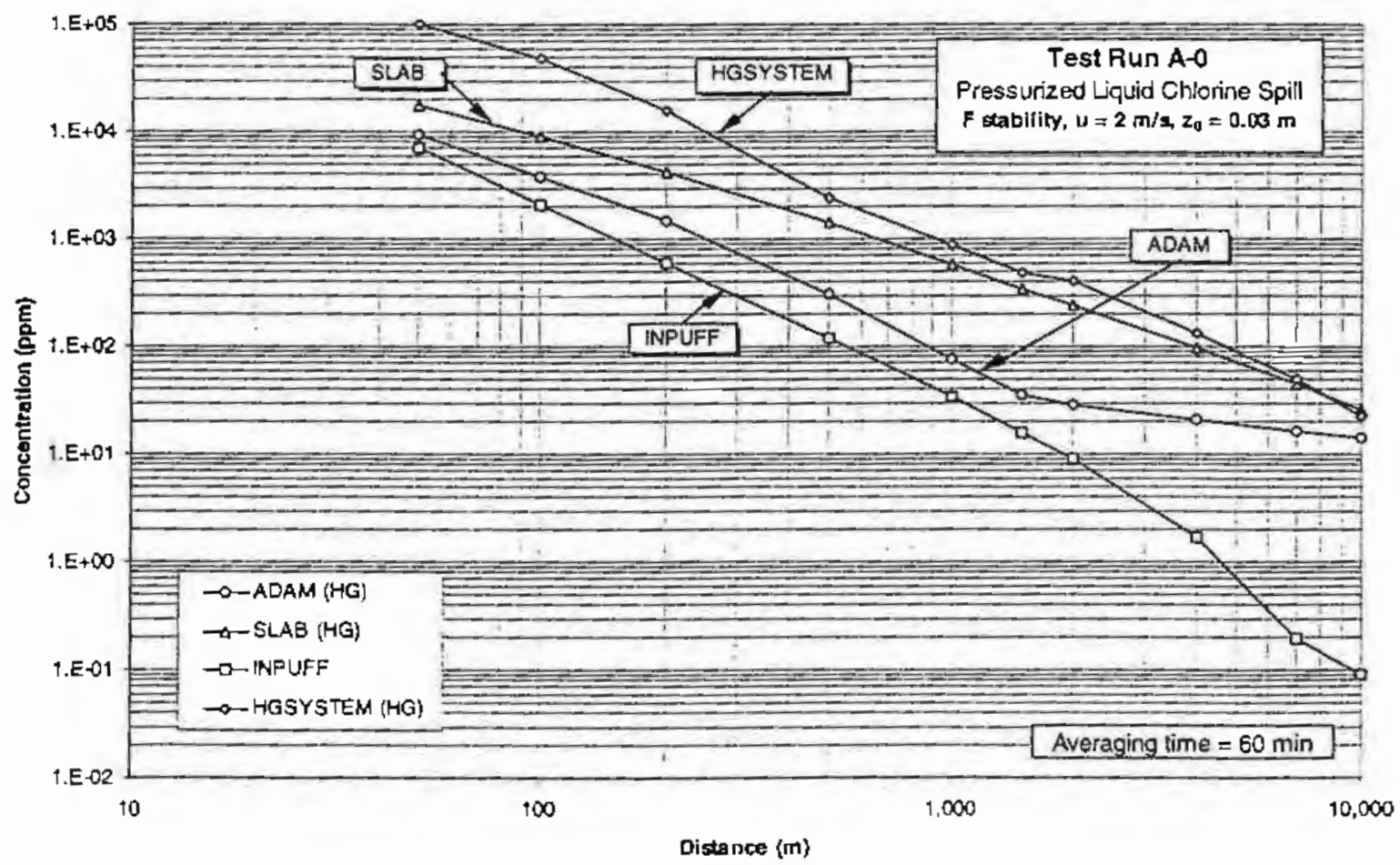

FIGURE 5.1f Base Case: Predicted Ground-Level Centerline Concentration vs. Downwind Distance for Transient Pool Evaporation - 60 Minute Average 


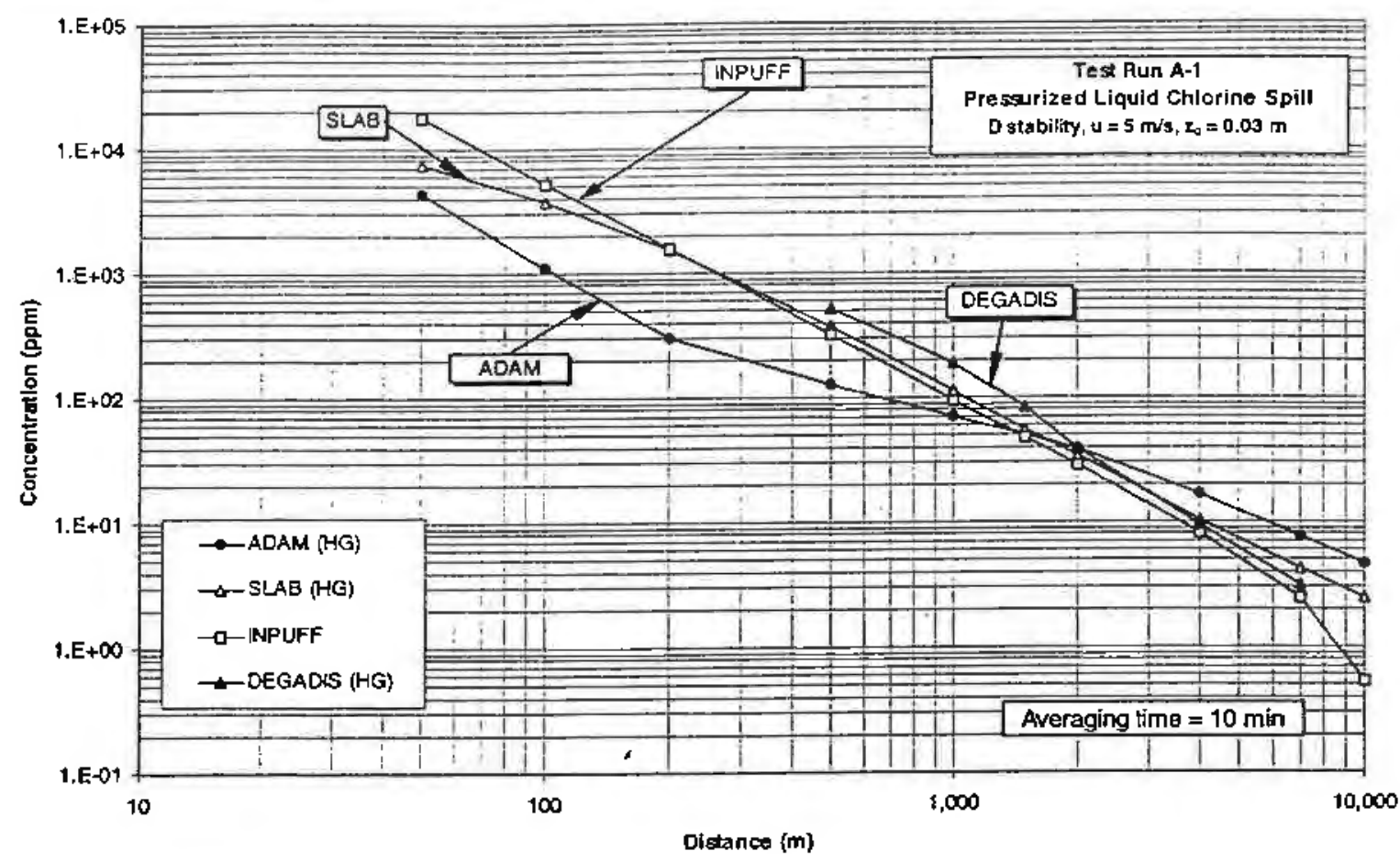

FIGURE 5.2 Variant 1: Predicted Ground-Level Centerline Concentration vs. Downwind Distance for Transient Pool Evaporation - Neutral Stability/Stronger Wind Speed

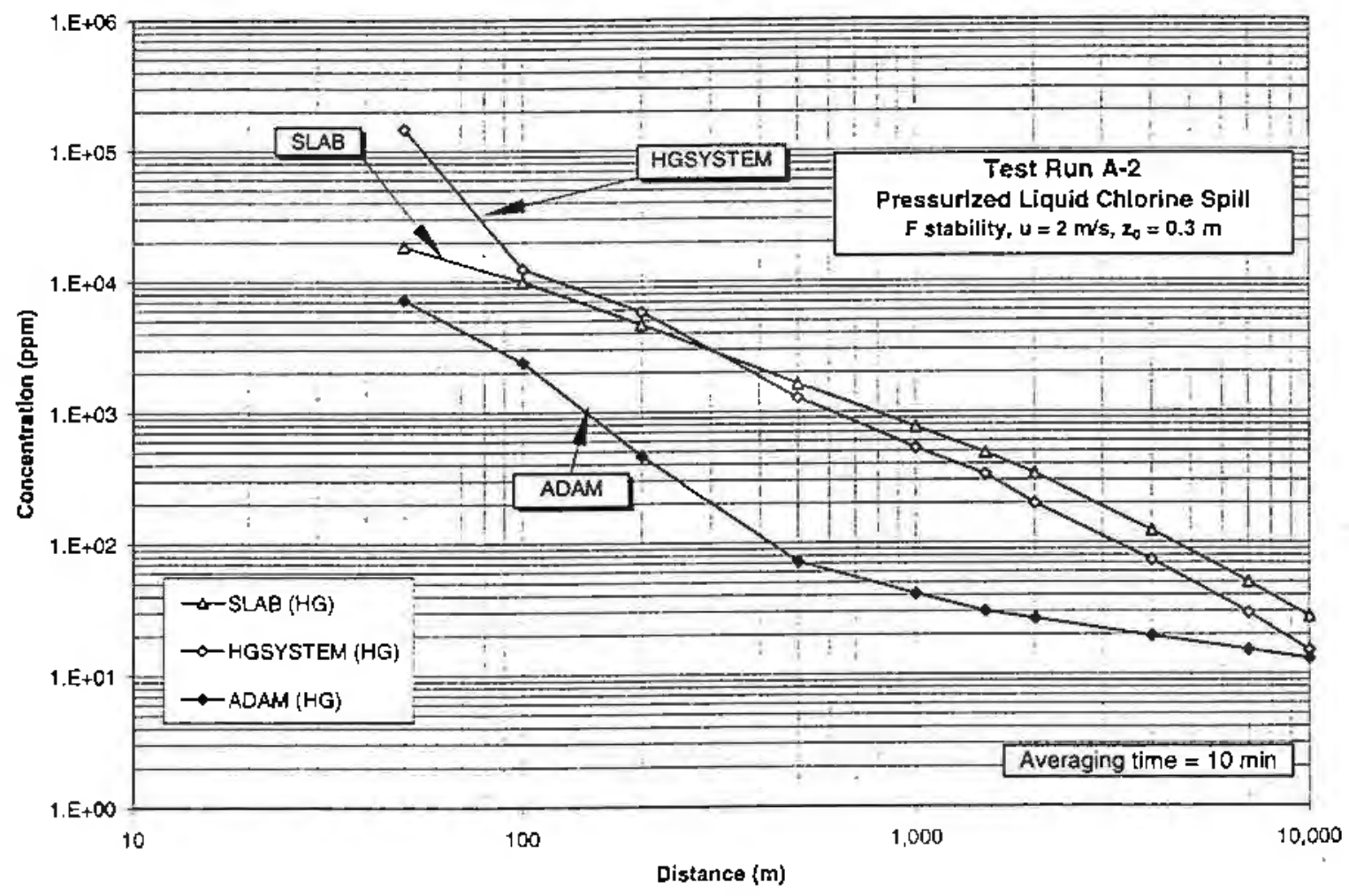

FIGURE 5.3 Variant 2: Predicted Ground-Level Centerline Concentration vs. Downwind Distance for Transient Pool Evaporation - Greater Surface Roughness 


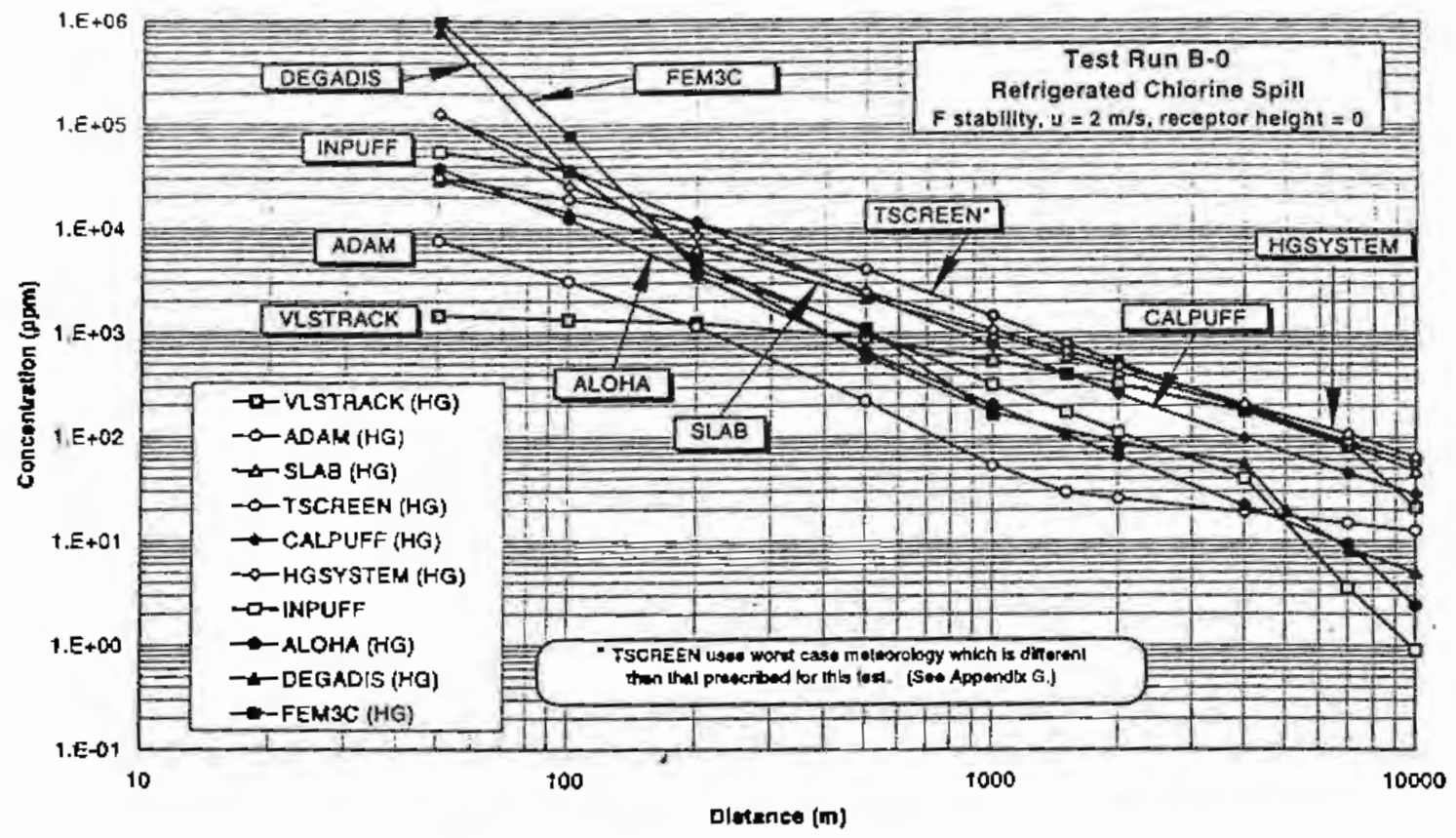

FIGURE 5.4a Base Case: Predicted Ground-Level Centerline Concentration vs. Downwind Distance from Steady Evaporating Pool (Moderately Stable Conditions, Wind Speed = $2 \mathrm{~m} / \mathrm{s}$, Ground-Level Receptor)

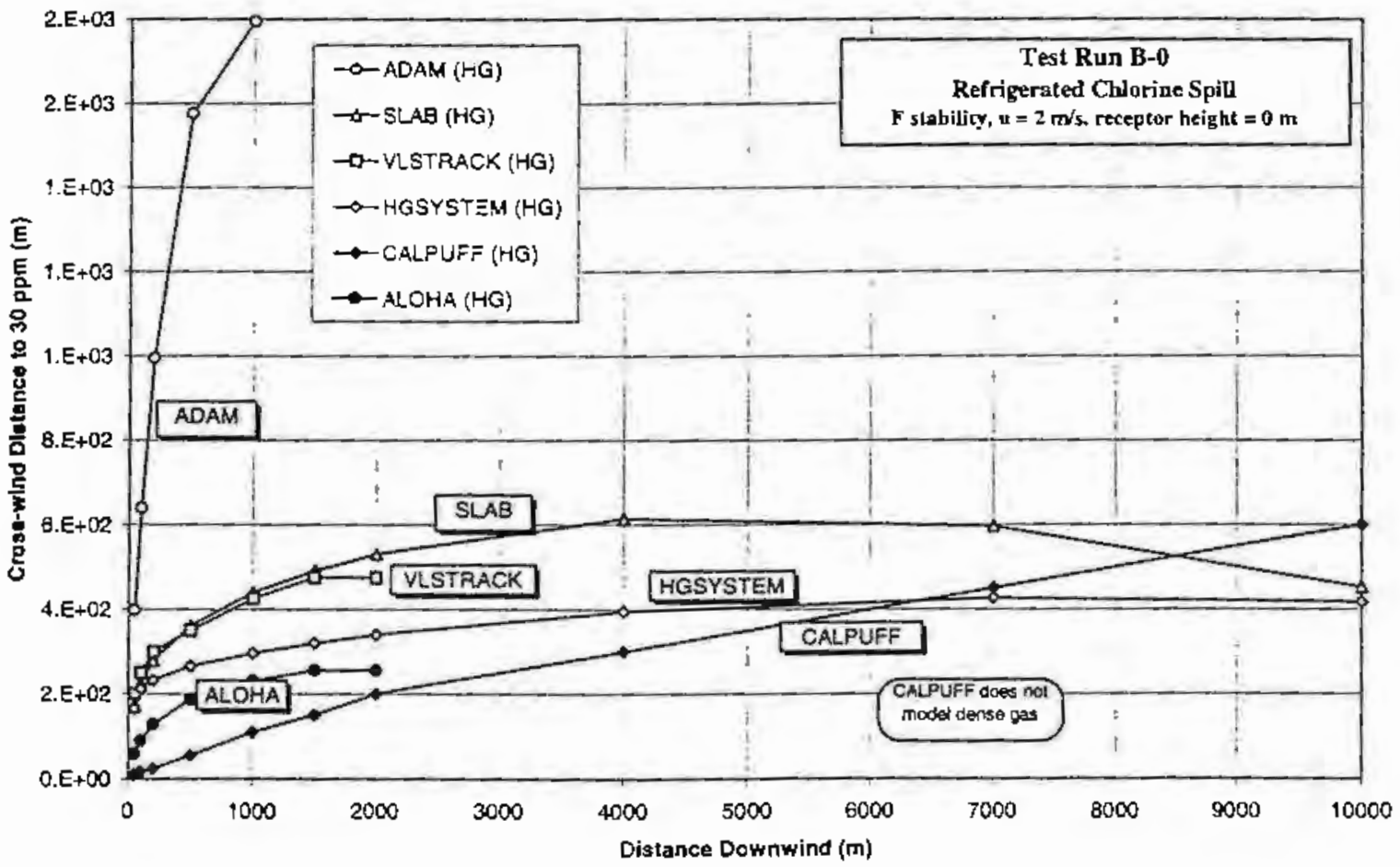

FIGURE 5.4b Base Case: Lateral Distance from Centerline to 30 ppm Prediction (Moderately Stable Conditions, Wind Speed $=2 \mathrm{~m} / \mathrm{s}$, Ground-Level Receptor) 


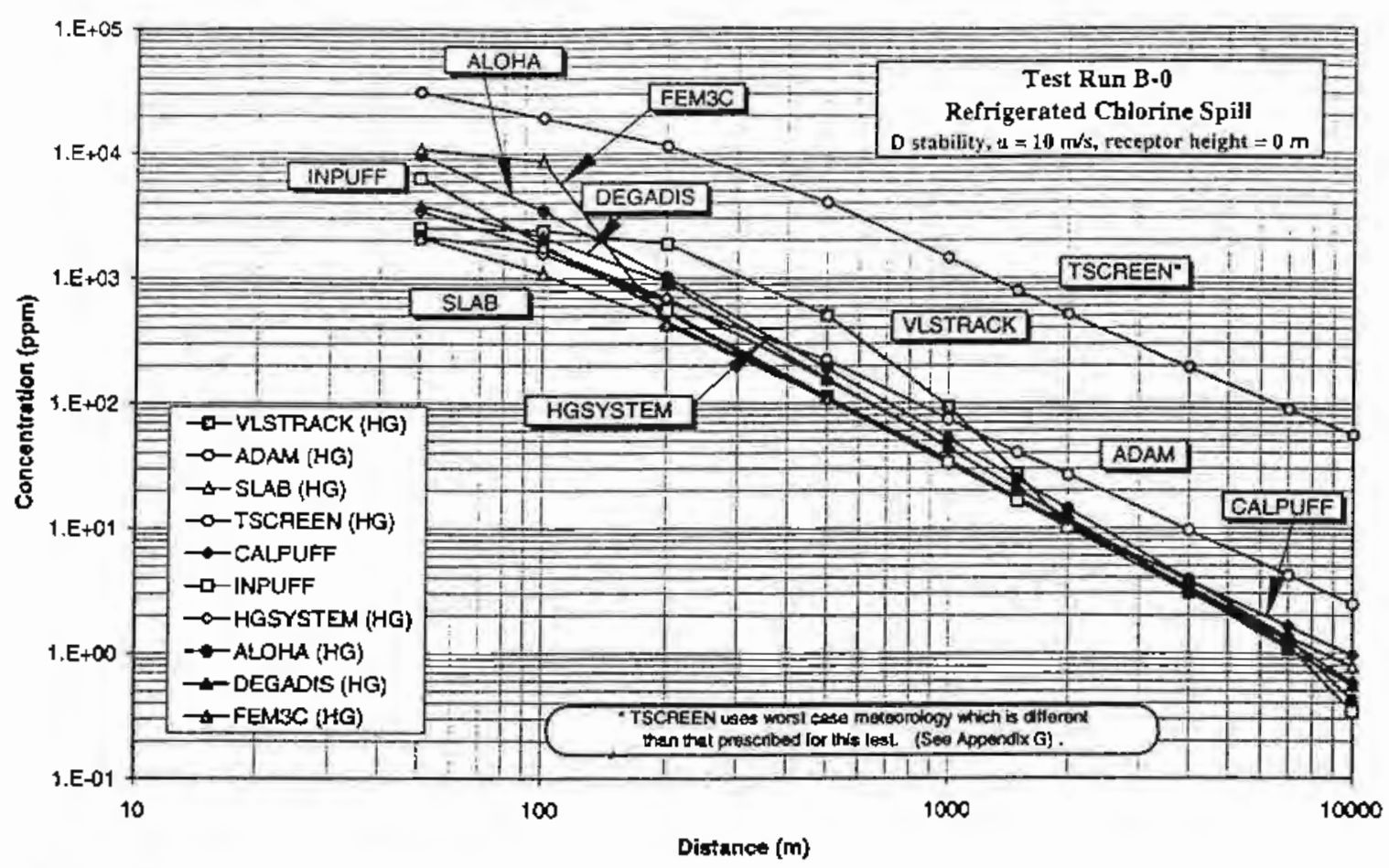

FIGURE 5.5a Variant 1: Predicted Ground-Level Centerline Concentration vs. Downwind Distance from Steady Evaporating Pool (Neutral Conditions, Wind Speed $=10 \mathrm{~m} / \mathrm{s}$, Ground-Level Receptor)

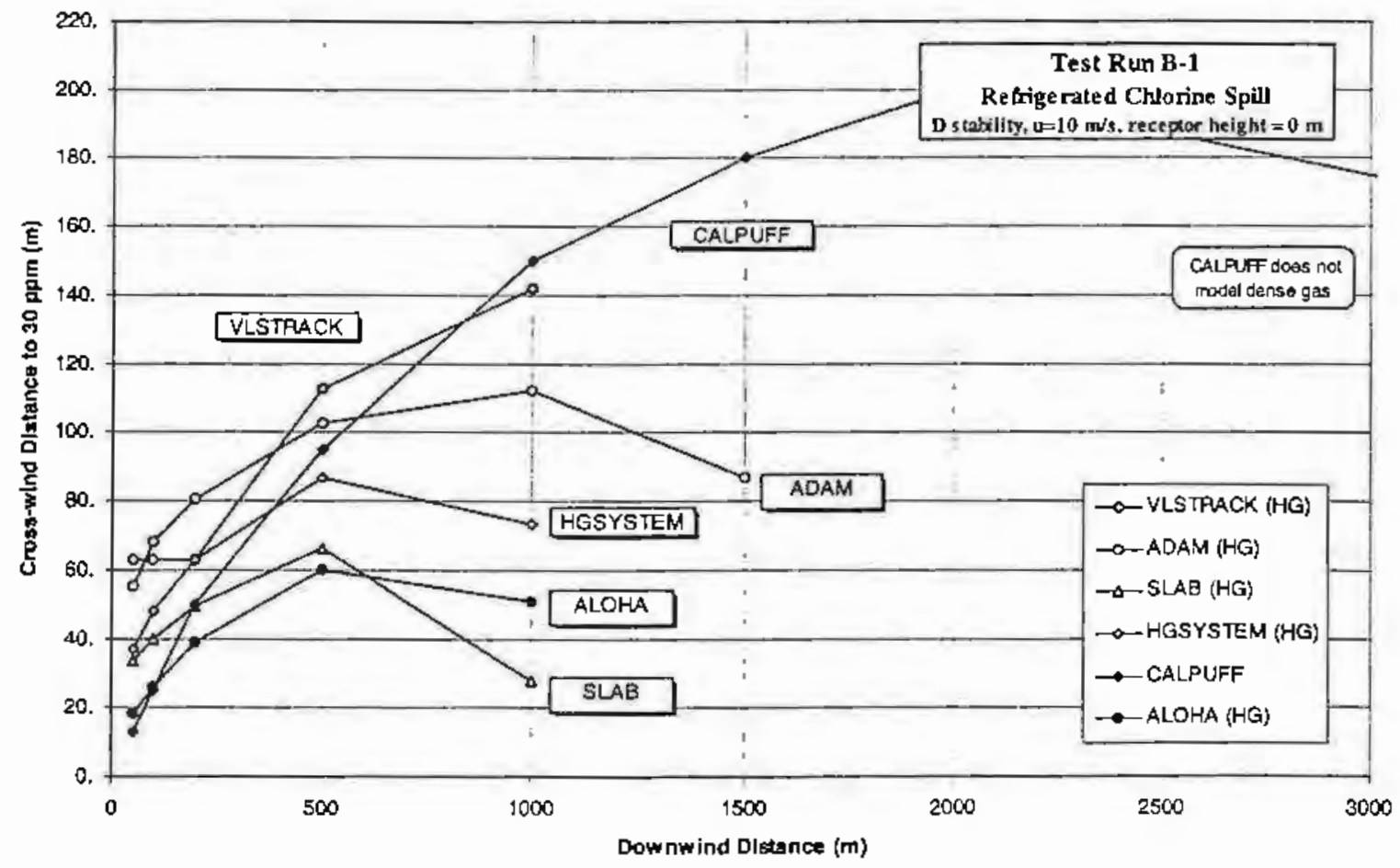

FIGURE 5.5b Variant 1: Lateral Distance from Centerline to $30 \mathrm{ppm}$ Prediction (Neutral Conditions, Wind Speed $=10 \mathrm{~m} / \mathrm{s}$, Ground-Level Receptor) 


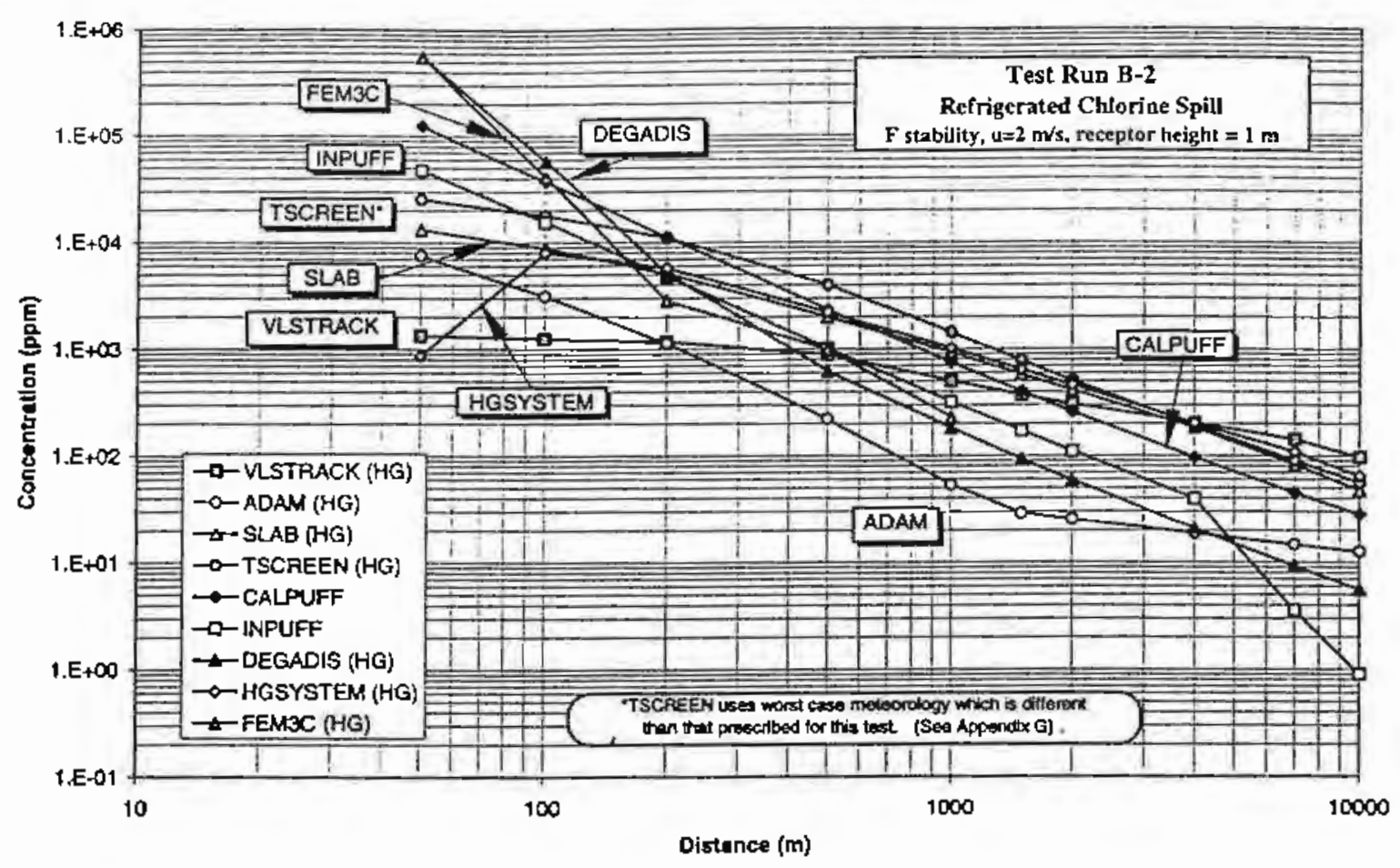

FIGURE 5.6a Variant 2: Predicted Ground-Level Centerline Concentration vs. Downwind Distance from Steady Evaporating Pool (Moderately Stable Conditions, Wind Speed $=2 \mathrm{~m} / \mathrm{s}$, Elevated Receptor)

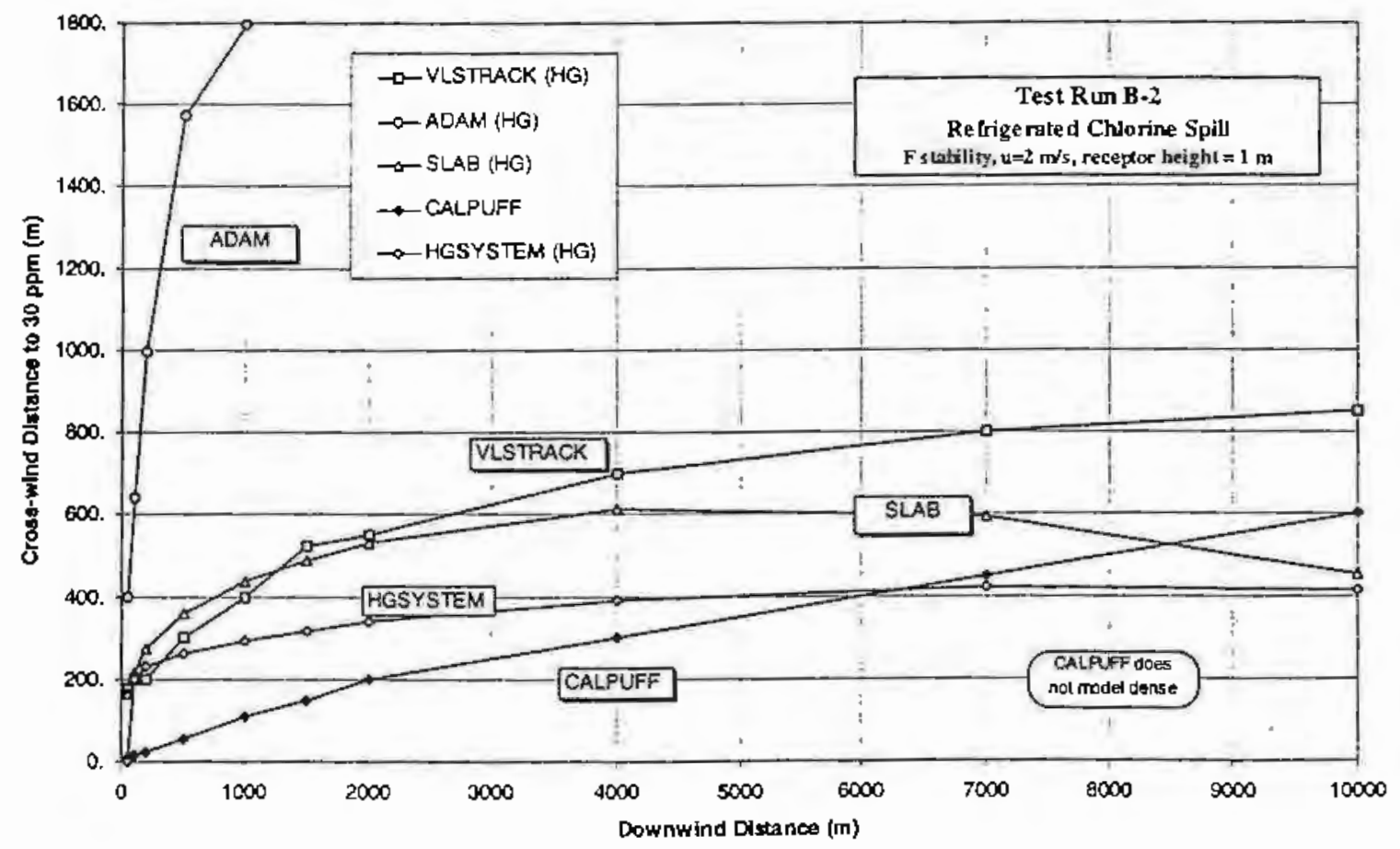

FIGURE 5.6b Variant 2: Lateral Distance from Centerline to $30 \mathrm{ppm}$ Prediction (Moderately Stable Conditions, Wind Speed $=2 \mathrm{~m} / \mathrm{s}$, Elevated Receptor) 


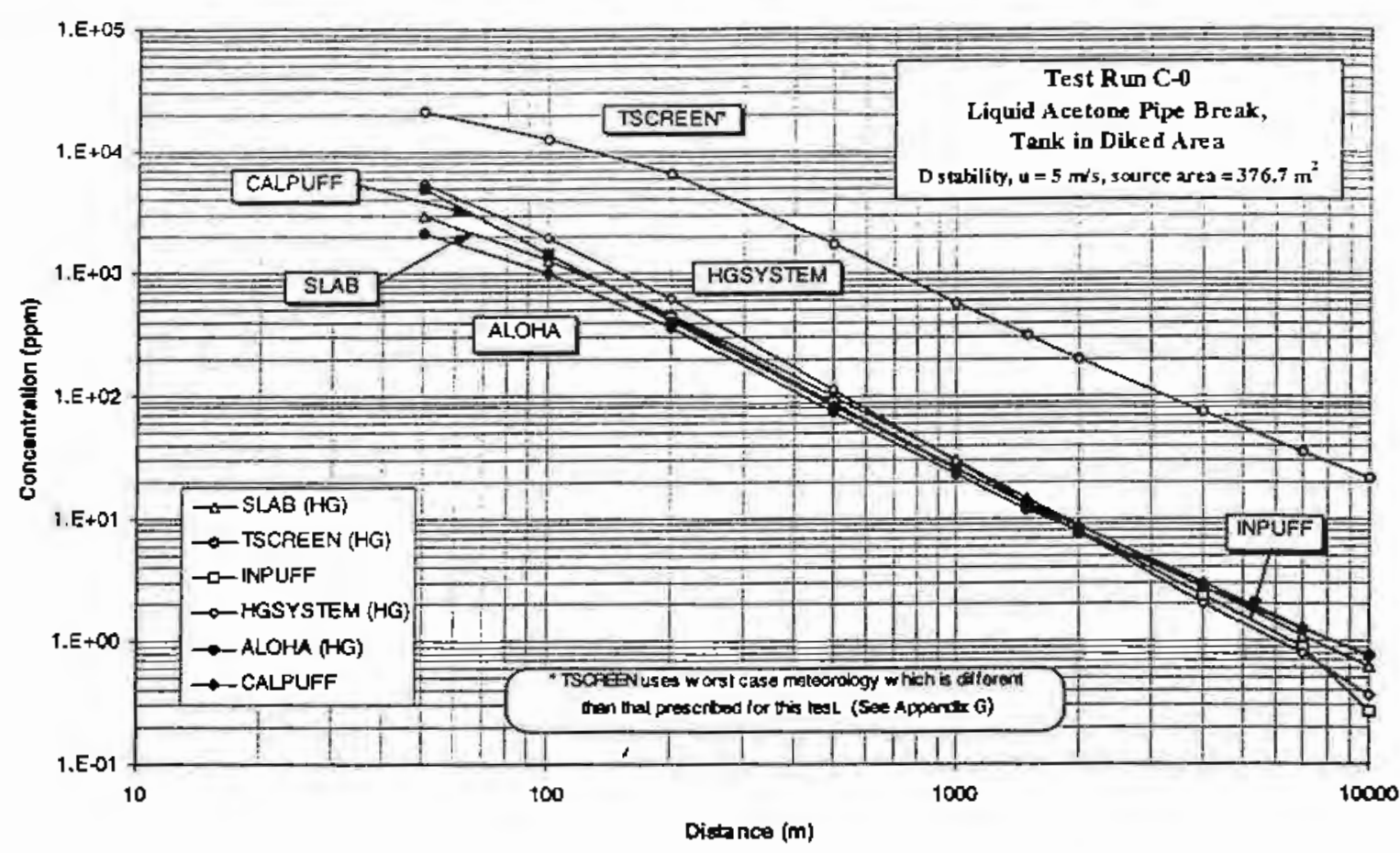

FIGURE 5.7 Base Case: Predicted Ground-Level Downwind Centerline Concentration vs. Downwind Distance from Slowly Evaporating Contained Liquid Spill (Neutral Conditions, Wind Speed $=5 \mathrm{~m} / \mathrm{s}$, Diked Area $=377 \mathrm{~m}^{2}$ )

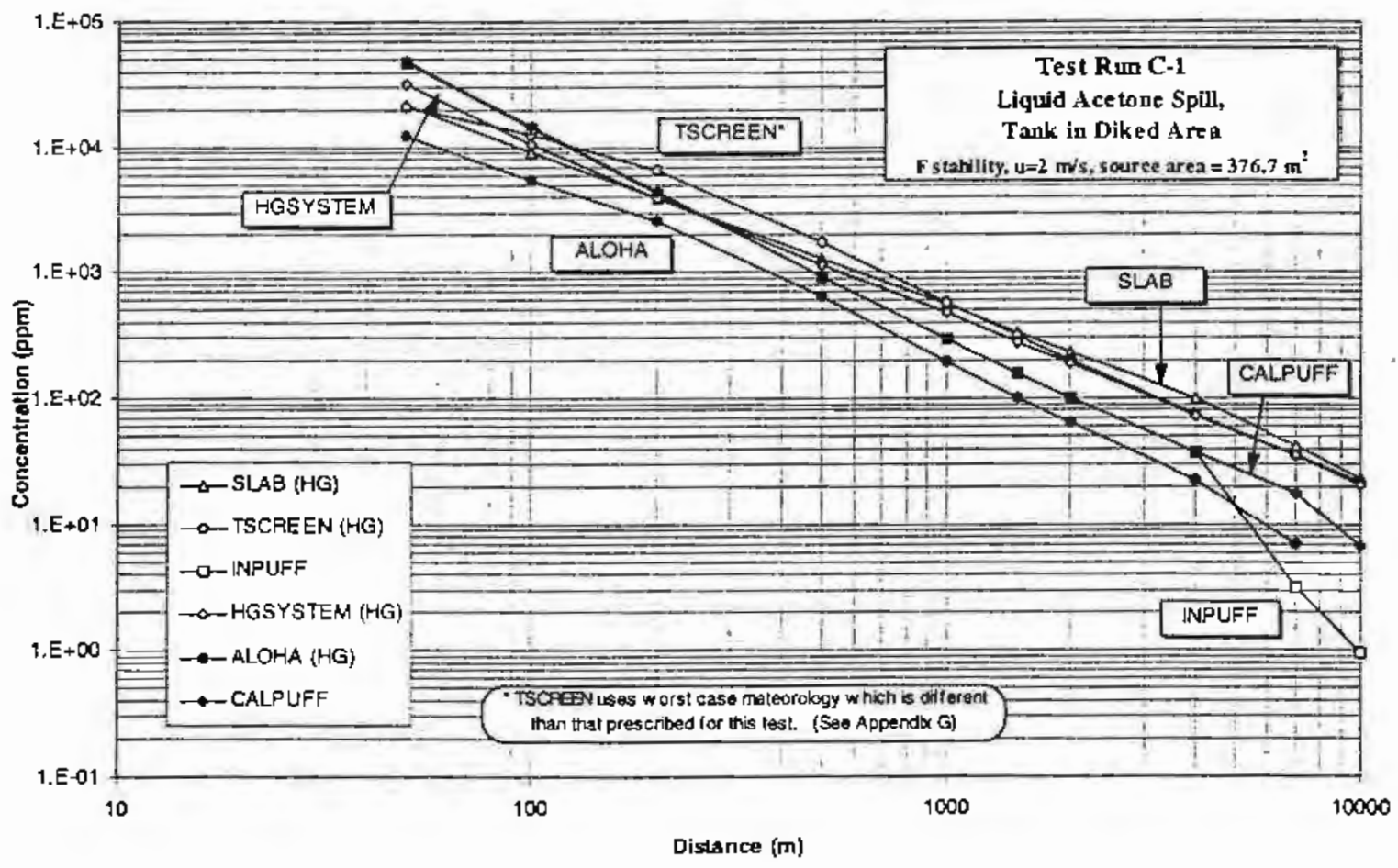

FIGURE 5.8 Variant 1: Predicted Ground-Level Downwind Centerline Concentration vs. Downwind Distance from Slowly Evaporating Contained Liquid Spill (Moderately Stable, Wind Speed = $2 \mathrm{~m} / \mathrm{s}$, Diked Area $=377 \mathrm{~m}^{2}$ ) 


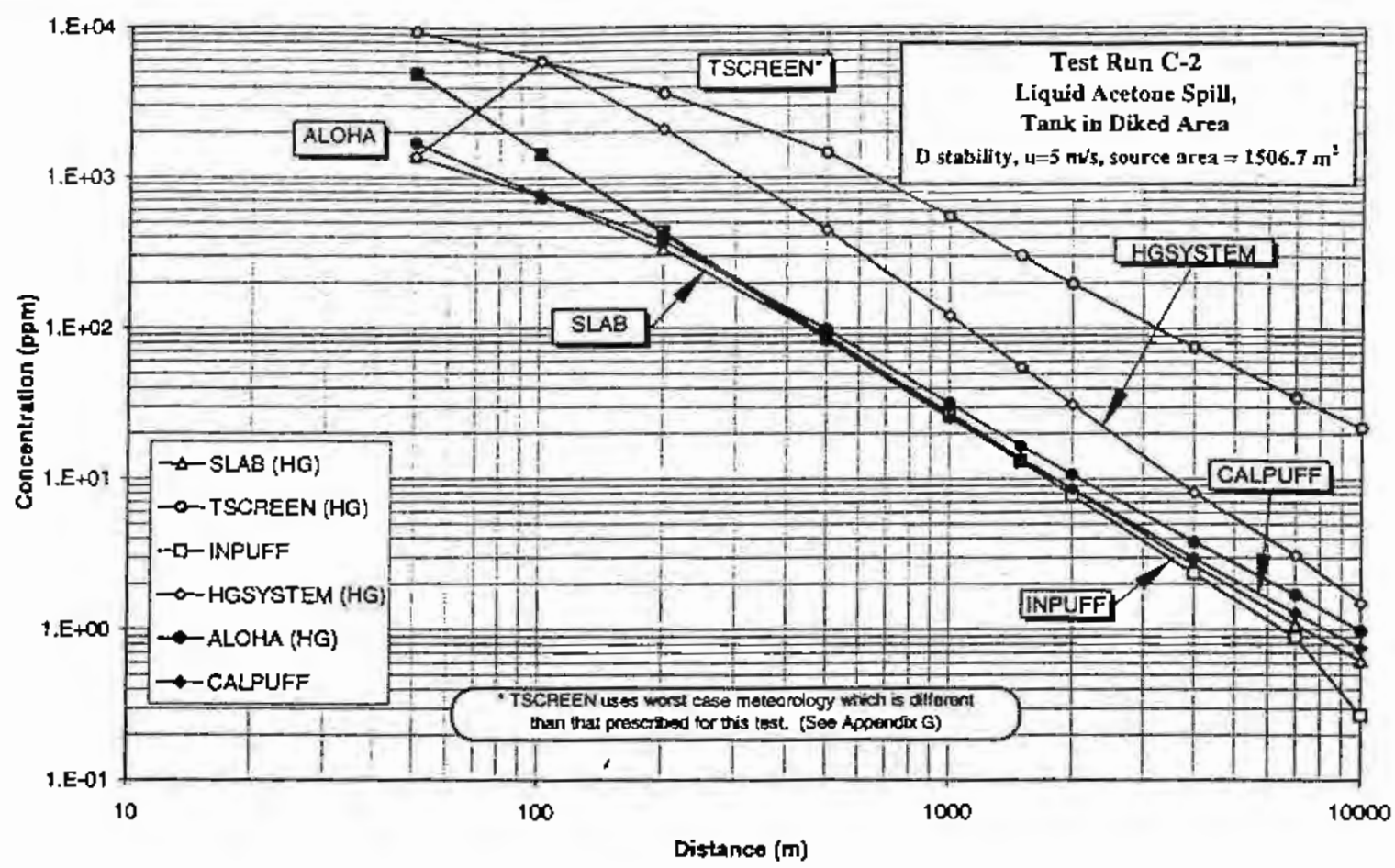

FIGURE 5.9 Variant 2: Predicted Ground-Level Downwind Centerline Concentration vs. Downwind Distance from Slowly Evaporating Contained Liquid Spill (Neutral Conditions, Wind Speed $=5 \mathrm{~m} / \mathrm{s}$, Diked Area $=1507 \mathrm{~m}^{2}$ )

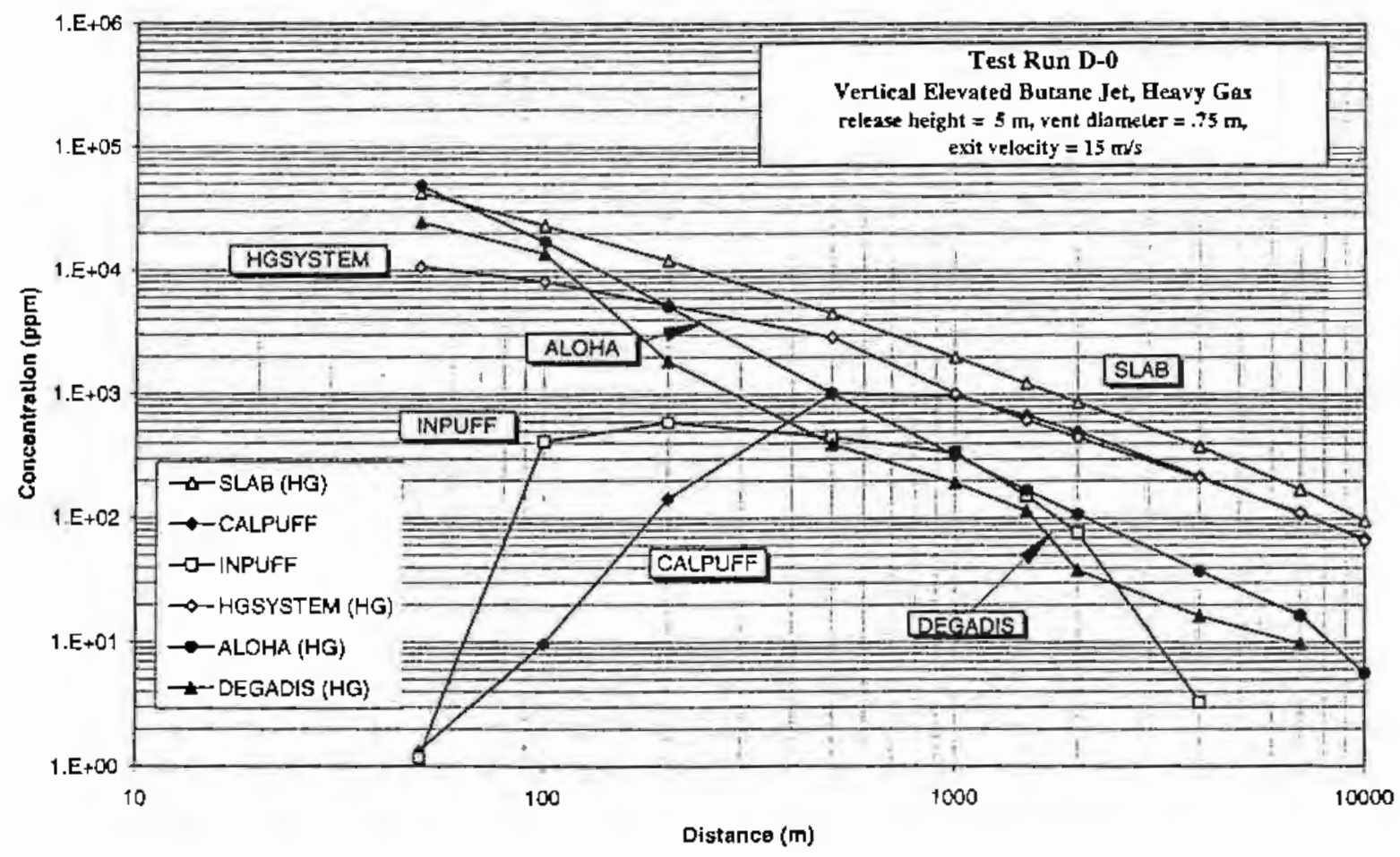

FIGURE 5.10 Base Case: Predicted Centerline Concentration vs. Downwind Distance from Vertical Momentum Jet (Moderately Stable, $\overrightarrow{\mathrm{u}}=2 \mathrm{~m} / \mathrm{s}, \mathrm{h}_{\mathrm{r}}=5 \mathrm{~m}, \mathrm{~d}_{\mathrm{v}}=75 \mathrm{~cm}$, $V_{Y}=15 \mathrm{~m} / \mathrm{s}$ ) 


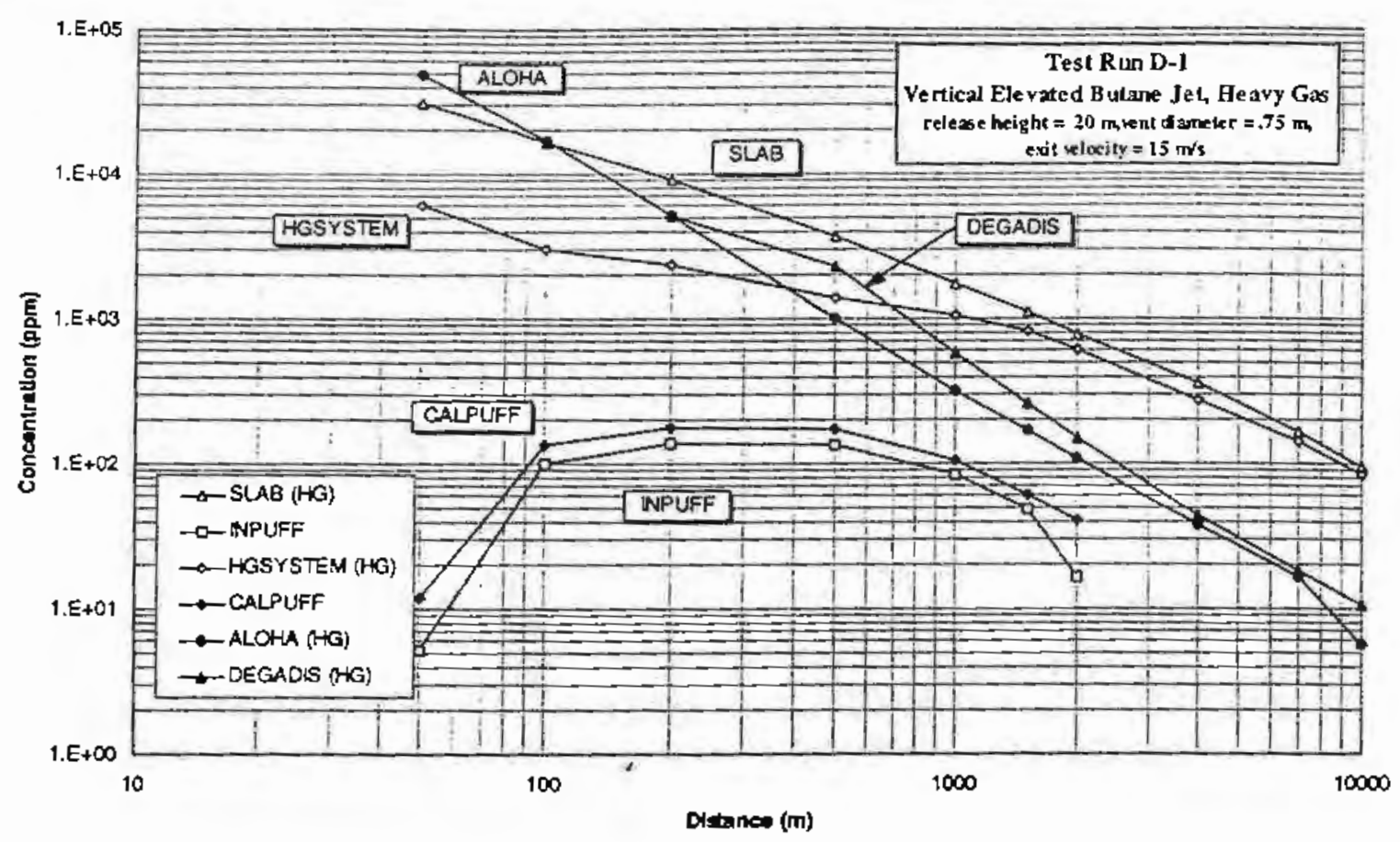

FIGURE 5.11 Variant 1: Predicted Centerline Concentration vs. Downwind Distance from Vertical Momentum Jet (Moderately Stable, $\bar{u}=2 \mathrm{~m} / \mathrm{s}, \mathrm{h}_{\mathrm{r}}=20 \mathrm{~m}, \mathrm{~d}_{\mathrm{v}}=75 \mathrm{~cm}$, $V_{v}=15 \mathrm{~m} / \mathrm{s}$ )

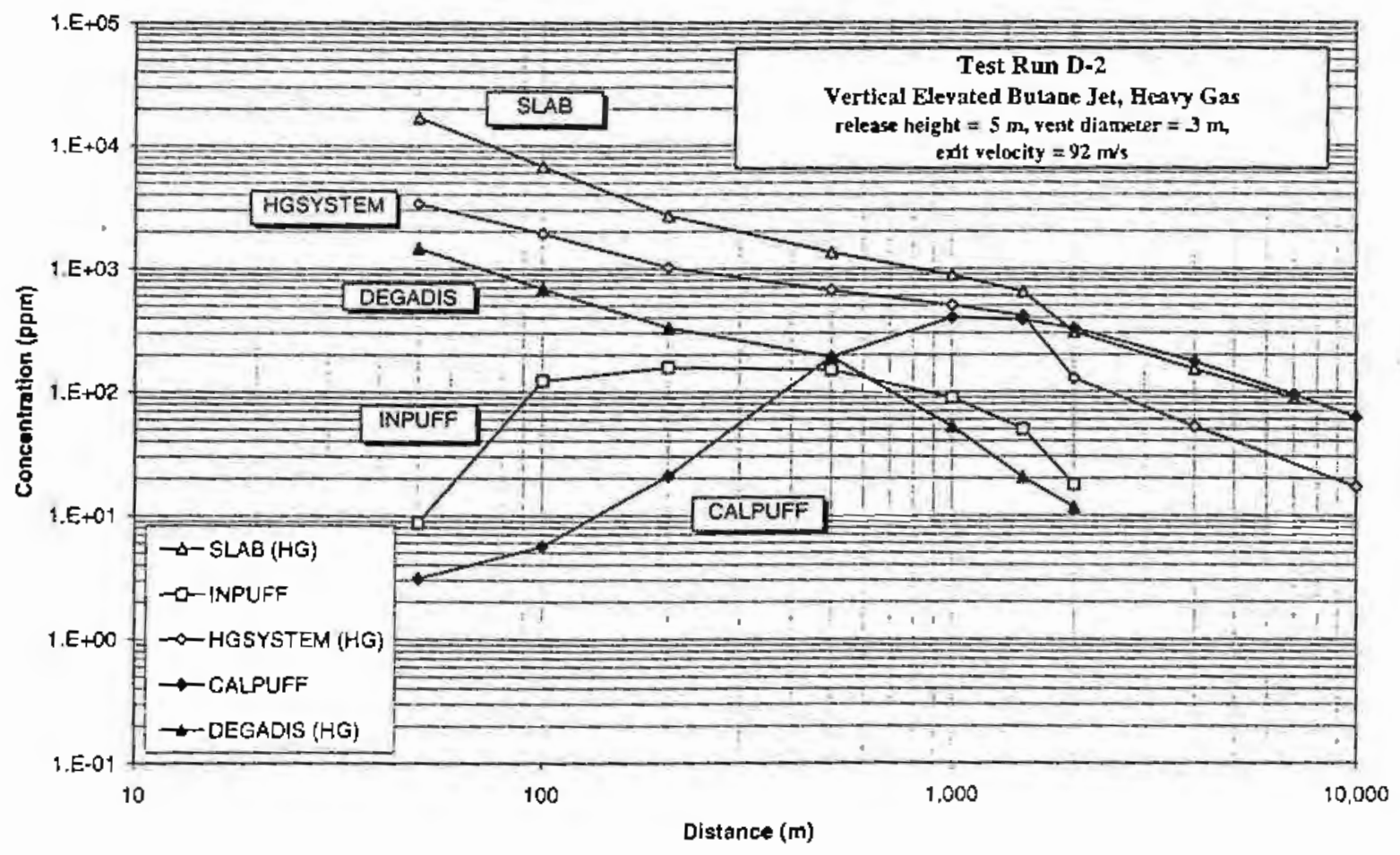

FIGURE 5.12 Variant 2: Predicted Centerline Concentration vs. Downwind Distance from Vertical Momentum Jet (Moderately Stable, $\bar{u}=2 \mathrm{~m} / \mathrm{s}, \mathrm{h}_{\mathrm{r}}=5 \mathrm{~m}, \mathrm{~d}_{\mathrm{v}}=30 \mathrm{~cm}$, $v_{v}=92 \mathrm{~m} / \mathrm{s}$ ) 


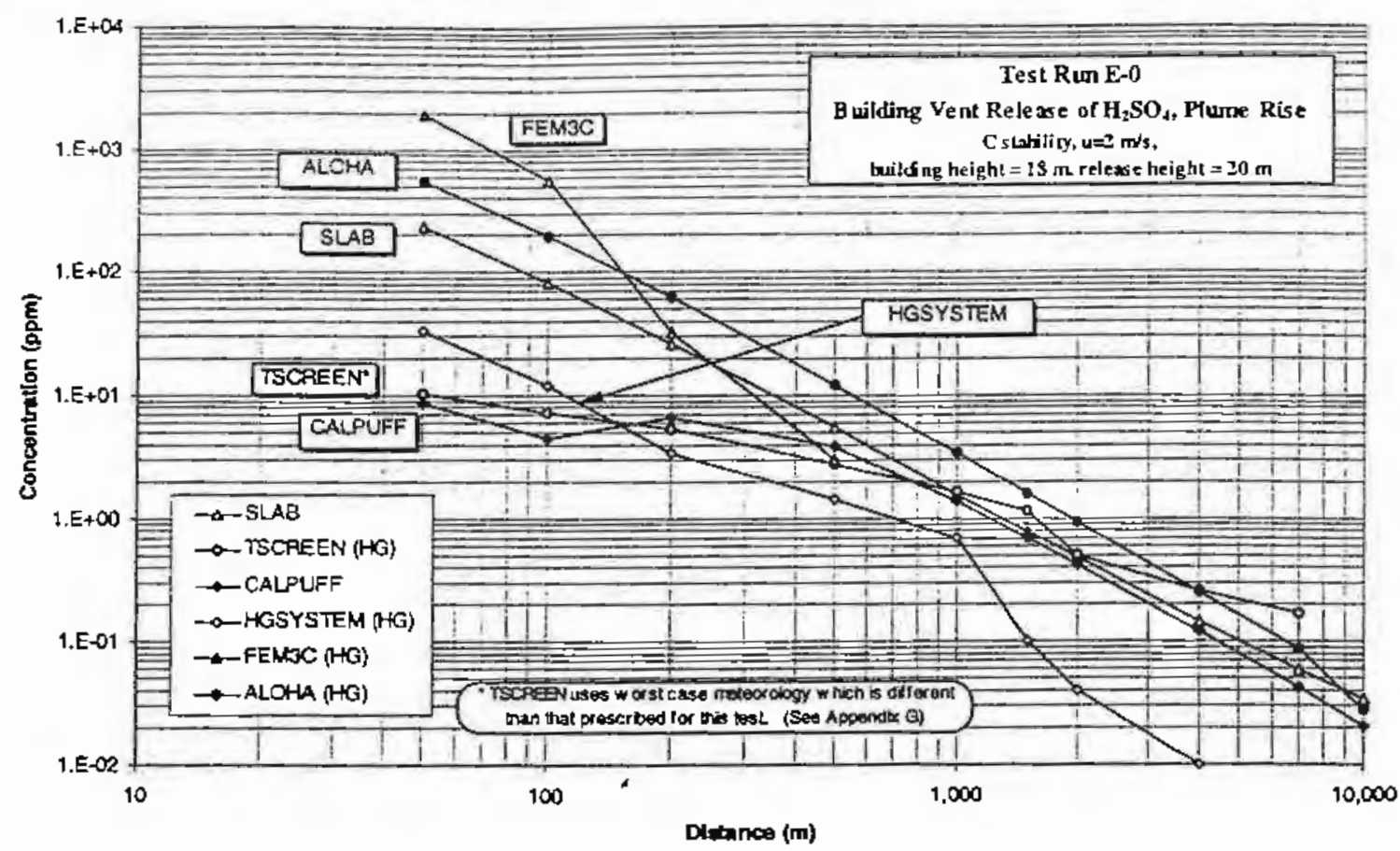

FIGURE 5.13 Base Case: Predicted Ground-Level Downwind Centerline Concentration vs. Downwind Distance from Buoyant Release (Slightly Unstable, $\bar{u}=2 \mathrm{~m} / \mathrm{s}, \mathrm{T}_{\mathrm{v}}=378 \mathrm{~K}$, $h_{r}=20 \mathrm{~m}, h_{b}=18 \mathrm{~m}, d_{v}=1 \mathrm{~m}, V_{\mathrm{v}}=6.4 \mathrm{~m} / \mathrm{s}$ )

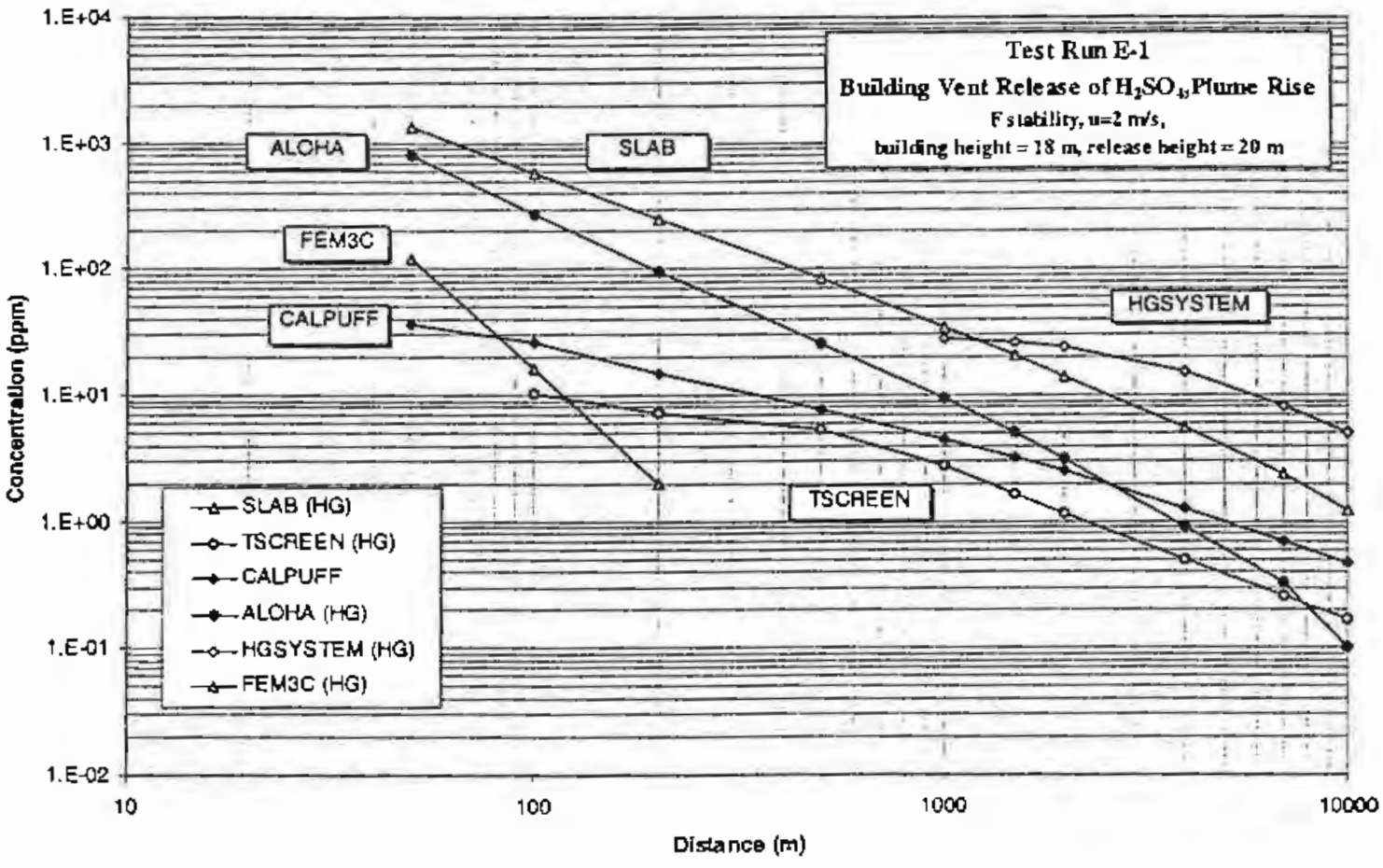

FIGURE 5.14 Variant 1: Predicted Ground-Level Downwind Centerline Concentration vs. Downwind Distance from Buoyant Release (Moderately Stable, $\overline{\mathrm{u}}=2 \mathrm{~m} / \mathrm{s}, \mathrm{T}_{\mathrm{v}}=378 \mathrm{~K}$, $h_{r}=20 \mathrm{~m}, h_{b}=18 \mathrm{~m}, d_{\mathrm{v}}=1 \mathrm{~m}, \mathrm{~V}_{\mathrm{v}}=6.4 \mathrm{~m} / \mathrm{s}$ ) 


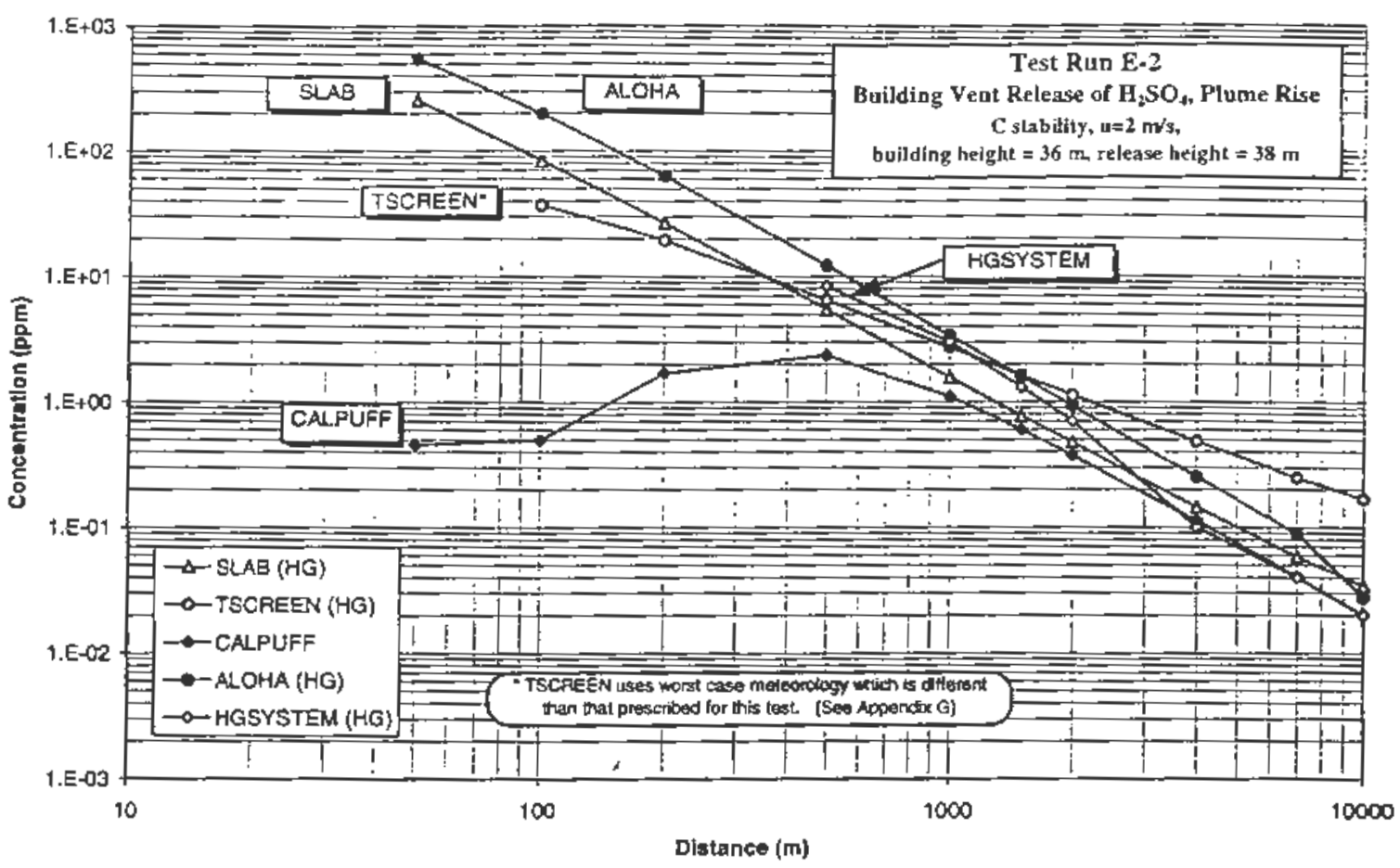

FIGURE 5.15 Variant 2: Predicted Ground-Level Downwind Centerline Concentration vs. Downwind Distance from Buoyant Release (Slightly Unstable, $\bar{u}=2 \mathrm{~m} / \mathrm{s}, \mathrm{T}_{\mathrm{V}}=378 \mathrm{~K}$, $h_{r}=38 \mathrm{~m}, h_{b}=36 \mathrm{~m}, \mathrm{~d}_{\mathrm{v}}=1 \mathrm{~m}, \mathrm{~V}_{\mathrm{Y}}=6.4 \mathrm{~m} / \mathrm{s}$ )

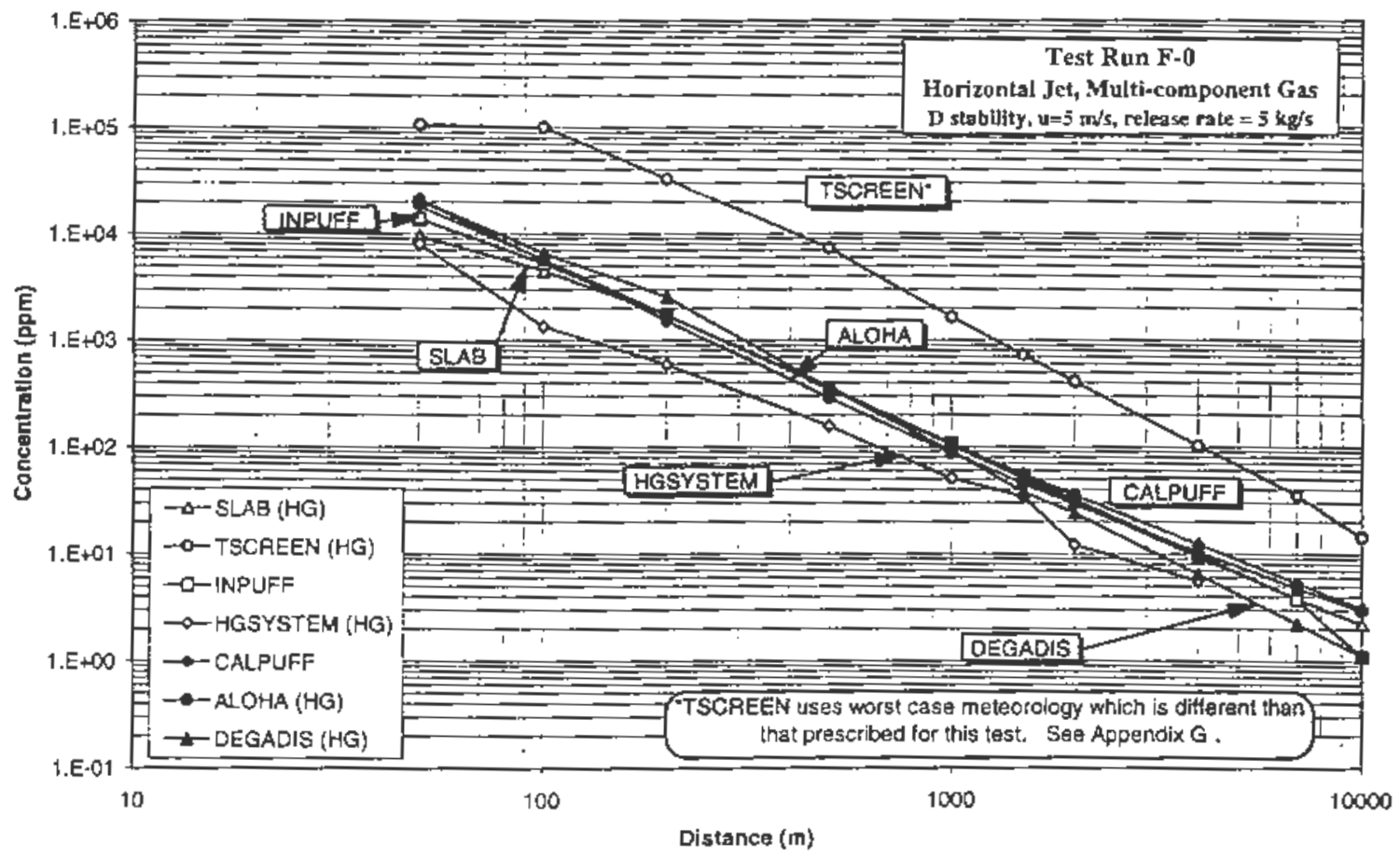

FIGURE 5.16 Base Case: Predicted Ground-Level Down wind Centerline Concentration vs. Downwind Distance from Horizontal Jet (Neutral Conditions, $\bar{u}=5 \mathrm{~m} / \mathrm{s}, Q=5 \mathrm{~kg} / \mathrm{s}$ ) 


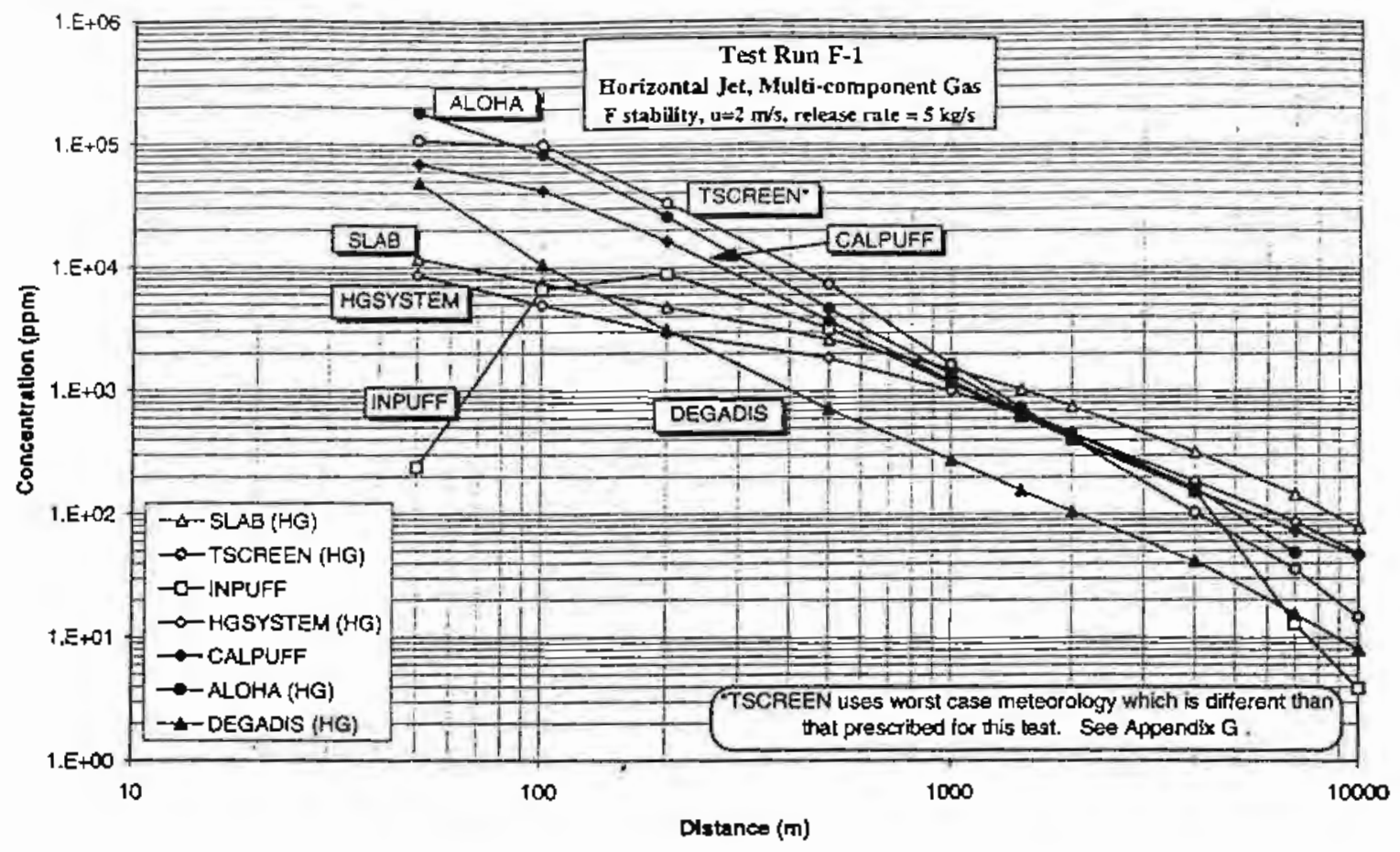

FIGURE 5.17 Variant 1: Predicted Ground-Level Downwind Centerline Concentration vs. Downwind Distance from Horizontal Jet (Moderately Stable, $\bar{u}=2 \mathrm{~m} / \mathrm{s}, \mathrm{Q}=5 \mathrm{~kg} / \mathrm{s}$ )

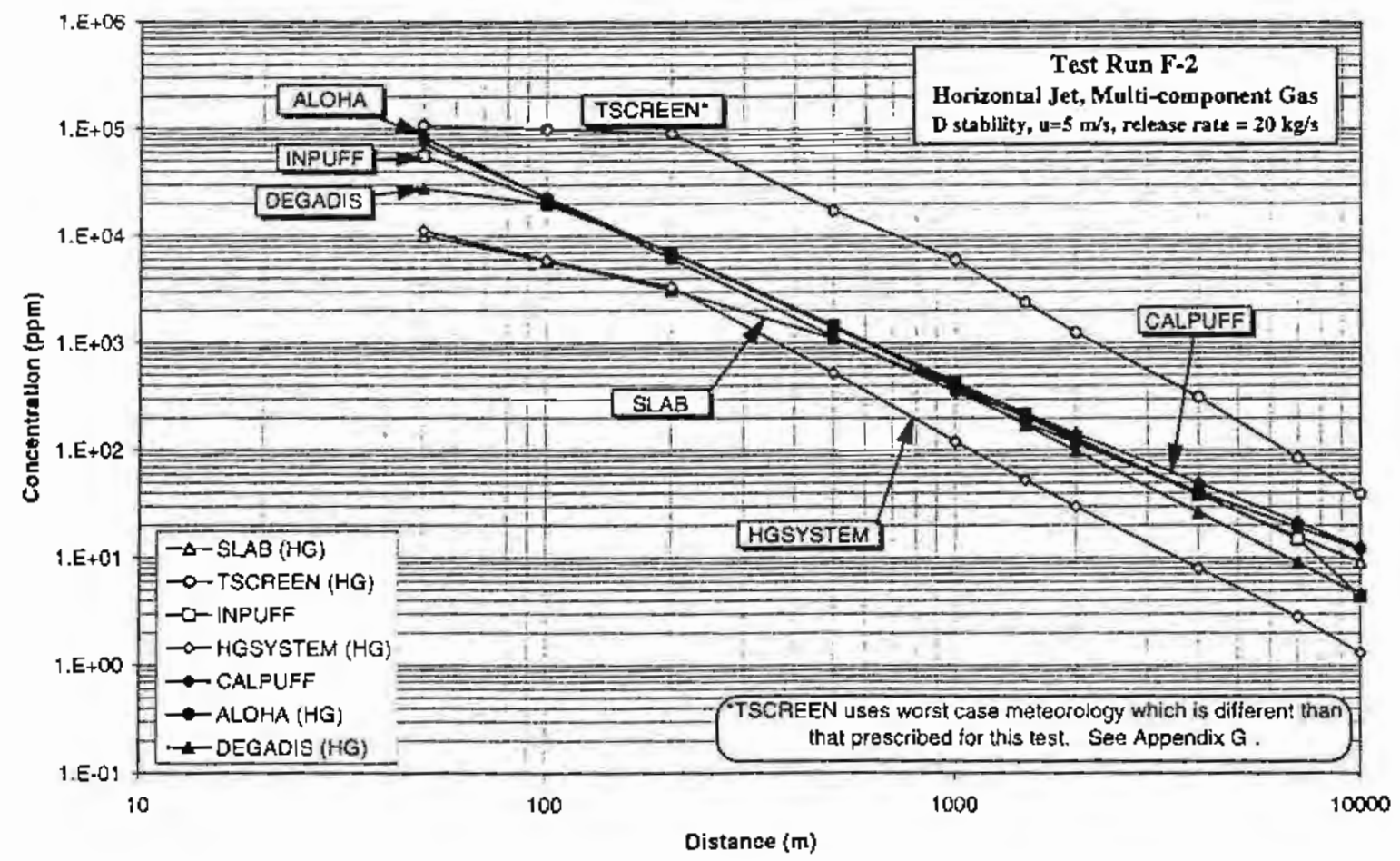

FIGURE 5.18 Variant 2; Predicted Ground-Level Downwind Centerline Concentration vs. Downwind Distance from Horizontal Jet (Neutral Conditions, $\vec{u}=5 \mathrm{~m} / \mathrm{s}, \mathrm{Q}=20 \mathrm{~kg} / \mathrm{s}$ ) 


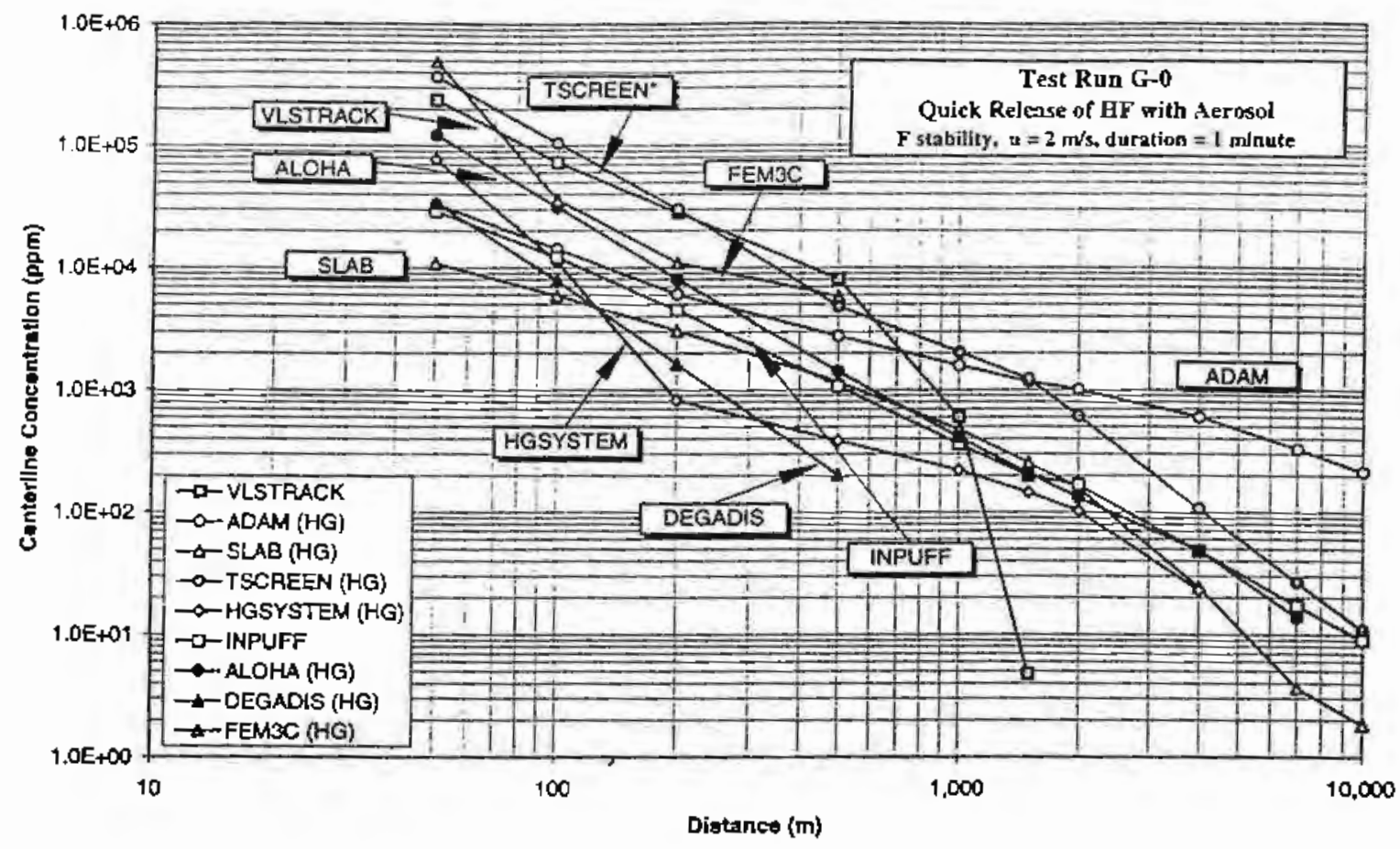

FIGURE 5.19a Base Case: Predicted Ground-Level Downwind Centerline

Concentration vs. Downwind Distance from Heavy Aerosol Release (Moderately Stable, Wind Speed $=2 \mathrm{~m} / \mathrm{s}, \mathrm{t}_{\mathrm{d}}=1 \mathrm{~min}$ )

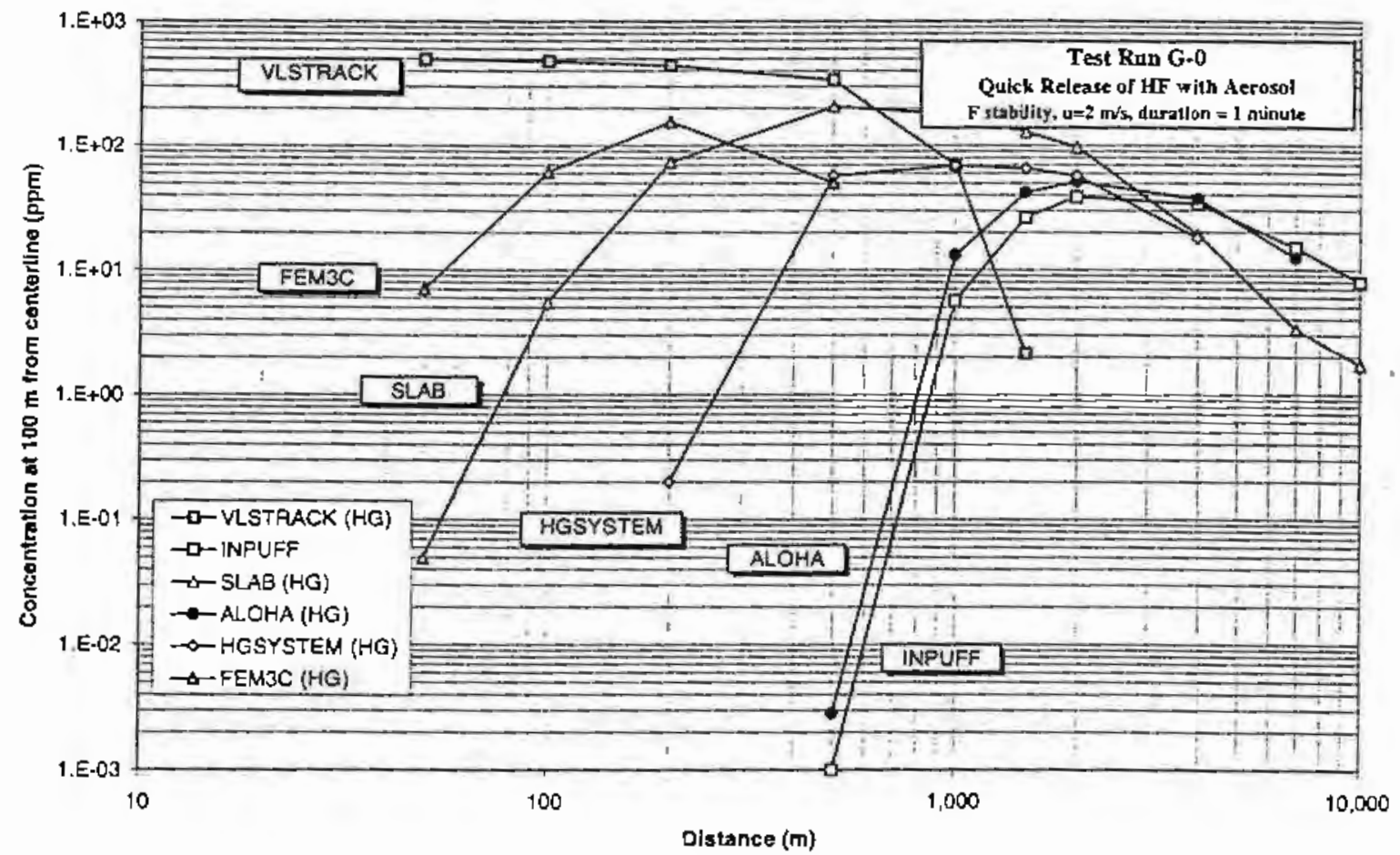

FIGURE 5.19b Base Case: Predicted Ground-Level Crosswind $100 \mathrm{~m}$ Centerline Concentration from Heavy Aerosol Release (Moderately Stable, Wind Speed $=2 \mathrm{~m} / \mathrm{s}$, $t_{d}=1 \mathrm{~min}$ ) 


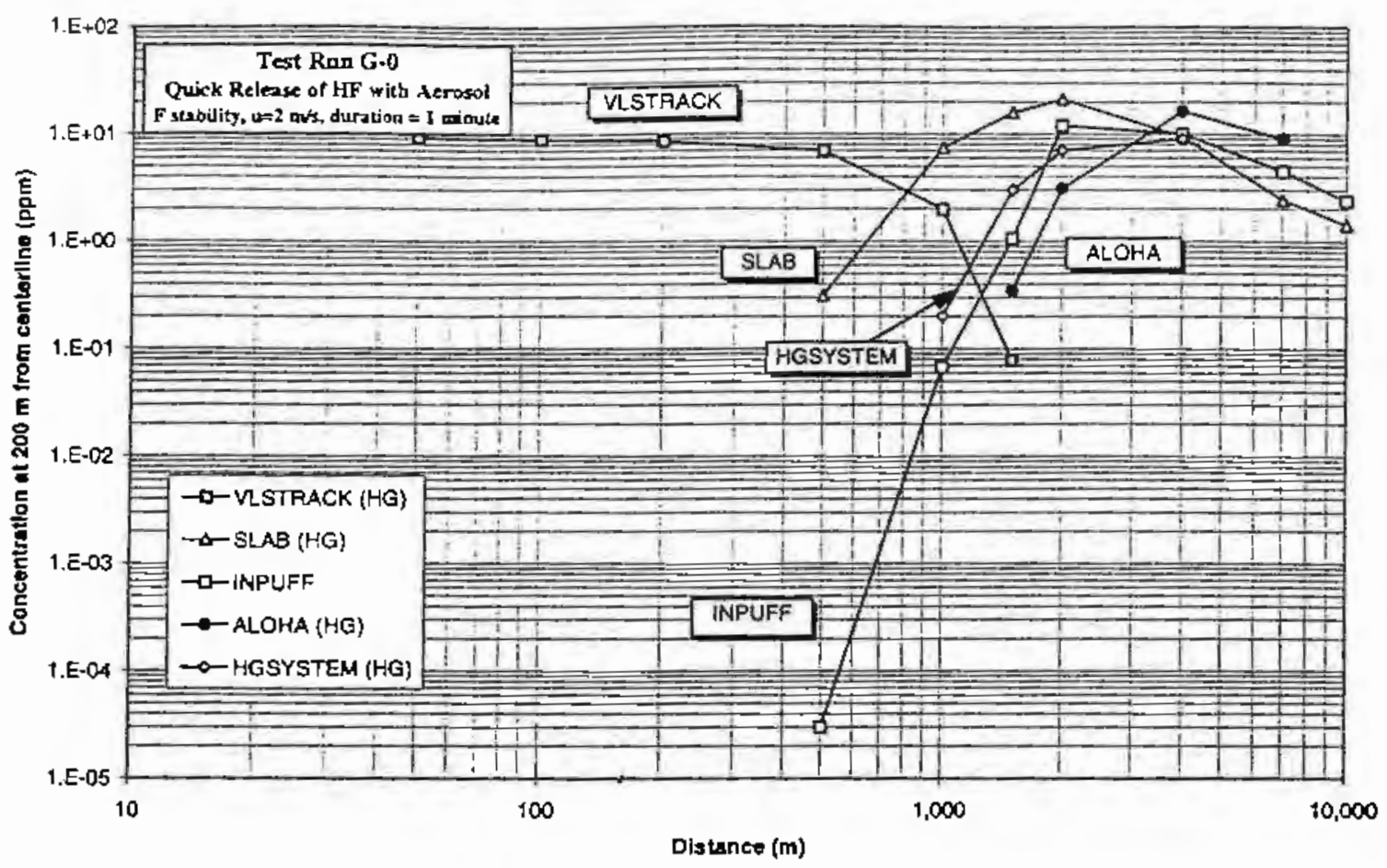

FIGURE 5.19c Base Case: Predicted Ground-Level Crosswind $200 \mathrm{~m}$ Centerline Concentration from Heavy Aerosol Release (Moderately Stable, Wind Speed $=2 \mathrm{~m} / \mathrm{s}$, $\mathrm{t}_{\mathrm{d}}=1 \mathrm{~min}$ )

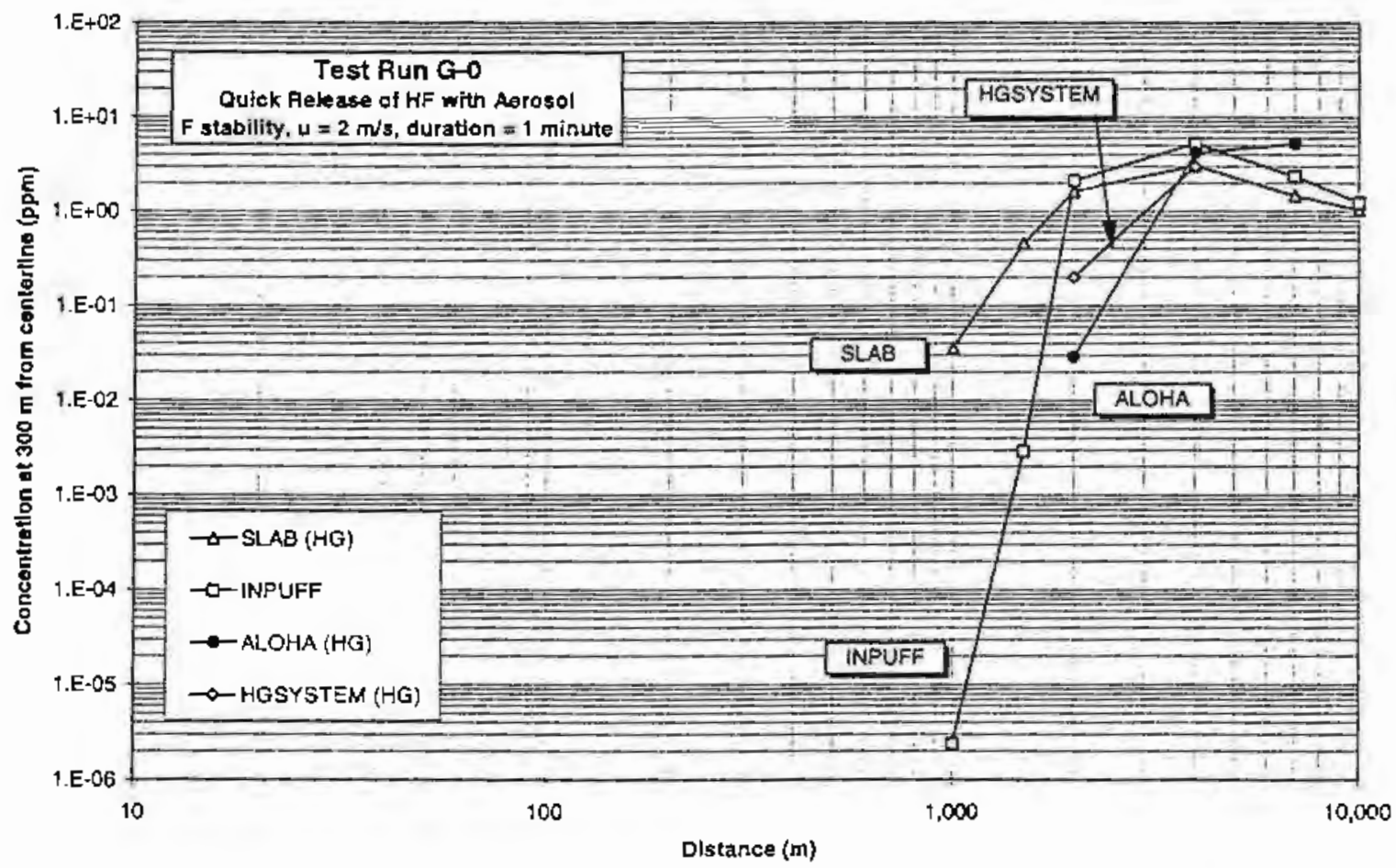

FIGURE 5.19d Base Case: Predicted Ground-Level Crosswind $300 \mathrm{~m}$ Centerline Concentration from Heavy Aerosol Release (Moderately Stable, Wind Speed $=2 \mathrm{~m} / \mathrm{s}$, $t_{d}=1$ min) 


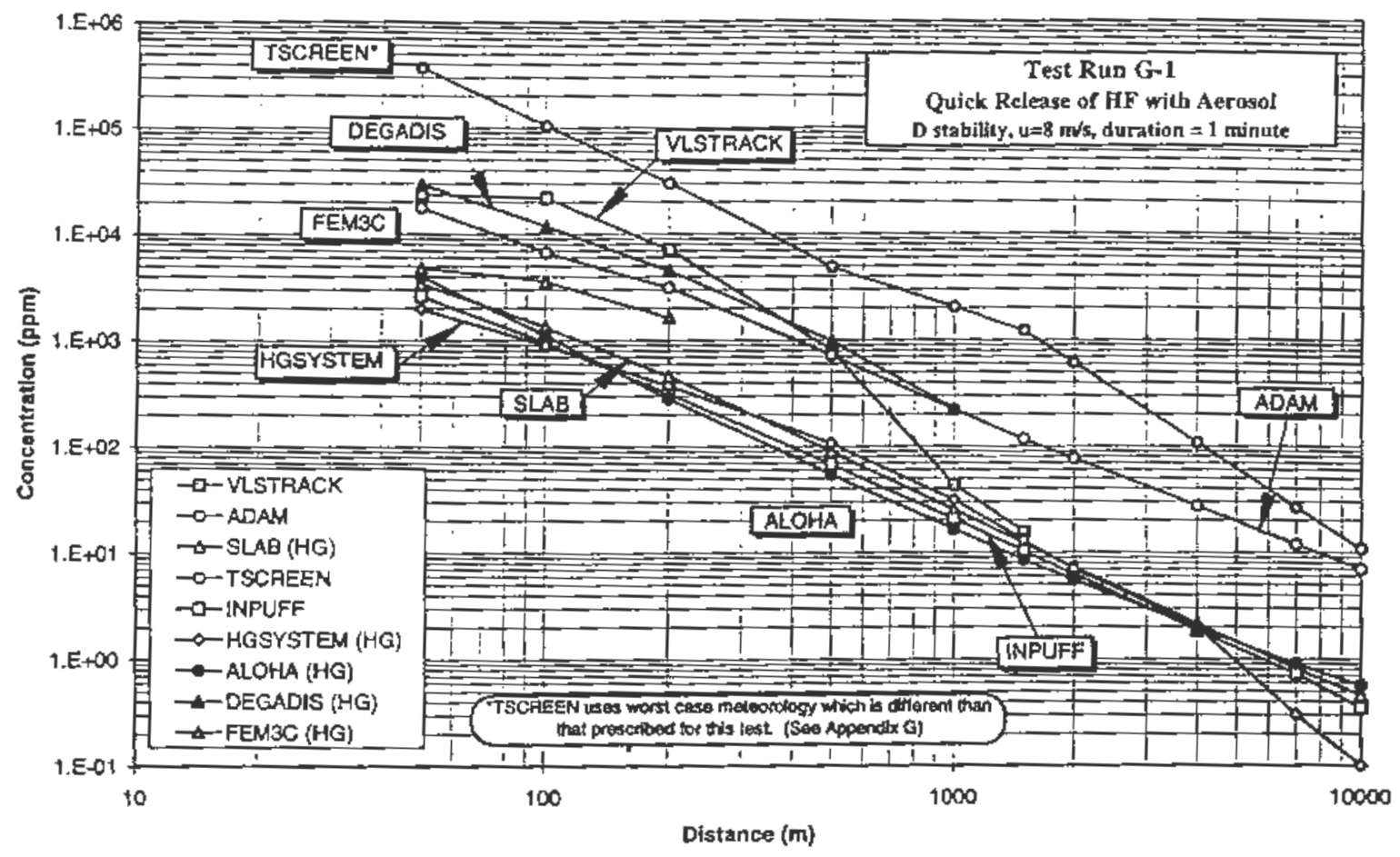

FIGURE 5.20 Variant 1: Predicted Ground-Level Downwind Centerline Concentration vs. Downwind Distance from Heavy Aerosol Release (Neutral Conditions, Wind Speed = $\left.8 \mathrm{~m} / \mathrm{s}, \mathrm{t}_{\mathrm{d}}=1 \mathrm{~min}\right)$

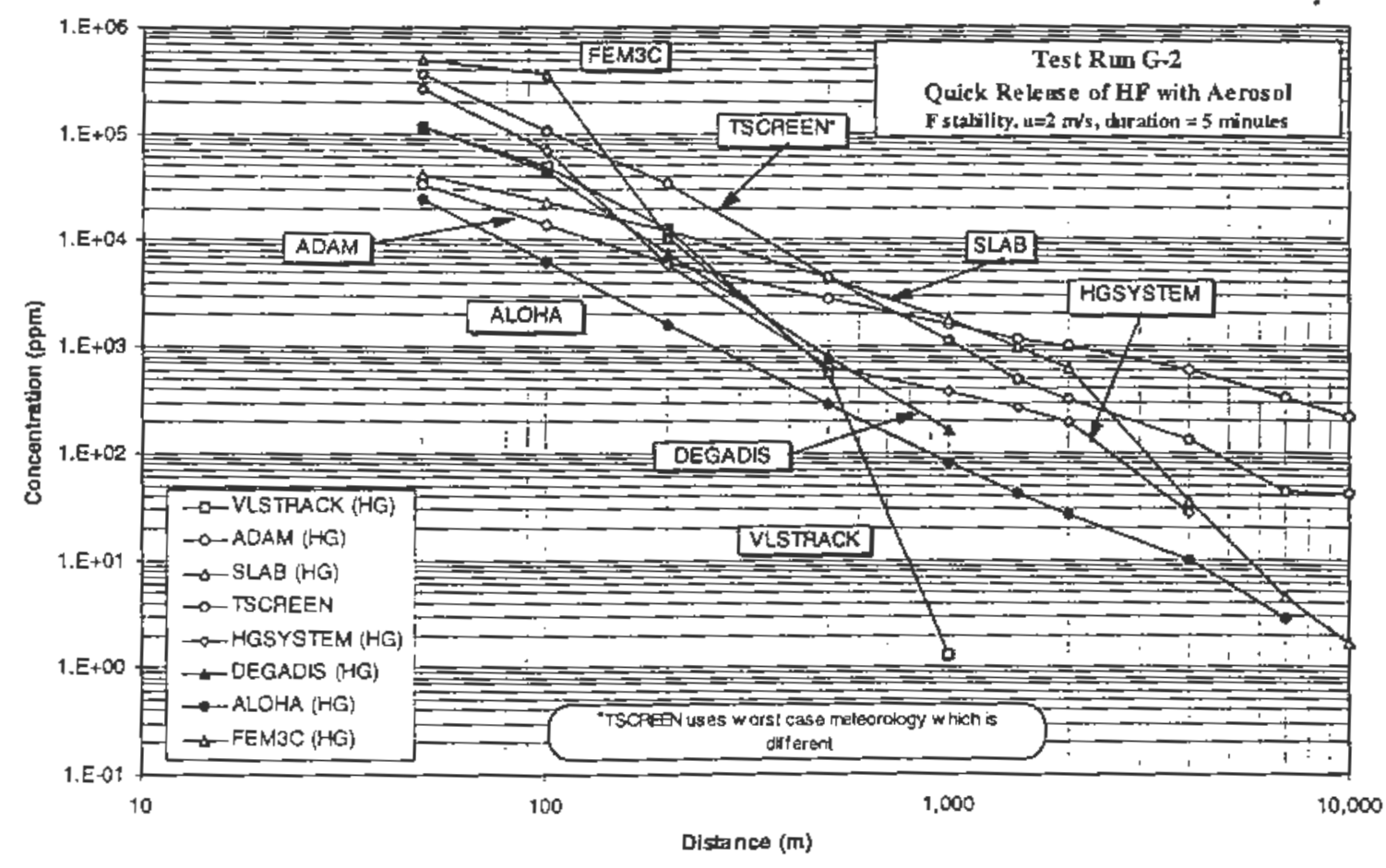

FIGURE 5.21 Variant 2: Predicted Ground-Level Downwind Centerline Concentration vs. Downwind Distance from Heavy Aerosol Release (Moderately Stable Conditions, Wind Speed $=2 \mathrm{~m} / \mathrm{s}, \mathrm{t}_{\mathrm{d}}=5 \mathrm{~min}$ ) 


\section{RECOMMENDATIONS}

\subsection{BACKGROUND}

Assessment of the possible consequences of a situation involving accidental release of a chemical to the atmosphere requires computations that could range from making simple hand or calculator estimates to applying advanced computer transport and dispersion models. Results from these computations will aid the regulatory (e.g., DOE Orders and standards, EPA rules) decision-making process pertaining to the safe operation of new or existing facilities within the DOE complex. In addition to estimating potential worker and/or public exposures and environmental impacts of chemical releases, such modeling may provide pertinent information for assessing siting options; designing a facility; planning emergency management and environment, safety, and health programs; and establishing operating conditions and procedures. The results of the modeling exercise may be used only within the DOE community of specialists, or they may undergo a broader review for discussion and assessment as part of external regulatory oversight procedures (e.g., accident prevention, process safety management, environmental impact assessment). Because of the importance of this information, the mission of the CDCA Working Group was to develop a list of recommended best practices for personnel conducting chemical-dispersion safety and consequence analyses at DOE facilities and to provide guidance on selection of the most appropriate methods and models for such analyses.

The guidelines provided in Section 6.2 are intended to provide general guidance on best practices for chemical accident safety analysis, along with more specific guidance related to consistent modeling procedures. Section 6.3 presents the working group's public domain model recommendations. Careful consideration was given to the Tier I and Tier II model reviews discussed earlier in this report. The recommendations are conditioned in terms of applicability of the evaluated models to a variety of facility, site location, and meteorologically constrained release conditions. The conditionally recommended models are not necessarily the only ones applicable or suitable for the specified applications, and the recommendations should not be considered endorsements. Finally, Section 6.4 identifies modeling needs and provides recommendations for strengthening and expanding the modeling tools available to the safety analyst for conducting scientifically sound and consistent consequence assessments. The ultimate goal is to improve the support necessary to ensure the protection of the health and safety of DOE facility workers and the public in the surrounding communities.

\subsection{GOOD MODELING AND SAFETY ANALYS1S PRACTICES}

Identification and application of best practices (i.e., good engineering, safety analysis, and modeling practices) are based in large part on experience in using dispersion models and understanding of the underlying physical processes involved in vapor cloud transport and diffusion. The objective of this section is to expand upon the modeling issues and themes repeatedly encountered when performing consequence assessments, including analysis of 
representative "design basis incident" (DBD) and "beyond design basis incident" (BDBI) 16 scenarios. The terms as used here are aralogous to, but not equivalent to, the terms altemative (formerly called more-likely) and worst-case release scenarios curently used in EPA guidance. ${ }^{17}$ The DBI and BDBI are chemical accident release scenarios with the most likely and the Iargest potential impact, respectively, that might credibly occur during facility operations. Contrary to conventional wisdom, the BDBI analysis is not always the simplest set of conditions to define. Like many other physical processes, the dispersion of an airbome contaminant can be affected by a host of physical parameters, and some of these parameters have competing effects in terms of mitigating or exacerbating the consequences associated with a release.

Recent guidance on the use of best practices in the selection and application of atmospheric dispersion models can be found in a policy statement issued by the Council of the Royal Meteorological Society (Britter et al. 1995). The working group established by this Council also provide recommendations for several modeling issues that should be considered in conducting and documenting a consequence assessment or modeling analysis in general. The report's modeling assessment documentation issues include the need for full discussion of analysis assumptions and the need for identification of how the analyst met the model input data requirements. Justification of model choice, sensitivity analysis, quality assurance, and model uncertainty and variability are also covered. Although the recommendations in that report are not explicitly incorporated into this document, the report is recommended as a good reference for conducting modeling in support of safety analysis documentation.

\subsubsection{General Guidance}

A number of general best practices should be considered in any dispersion analysis. These practices, along with general guidance provided in the Spills Working Group report (Brereton et al. 1996) and the general guidance referred to in the policy statement referenced above, are recommended in conducting consistent assessments for chemical safety basis documentation. The following are among the more crucial elements that should be taken into account:

1. Dispersion analyses conducted in support of SARs and other applications should use at least one set of BDBI release conditions for each accident scenario to determine the maximum possible impact on receptors of interest. A more detailed discussion of modeling assumptions for determining DBI and BDBI conditions is provided in Section 6.2.2.

16 DBI and BDBI are terms used in this report to distinguish recommended best practices specific or typical to chemical accident asscssments from established or recommend best practices specific or typical to radiological accident assessments.

17 Terms used in EPA's guidance for off-site consequence analysis in support of compliance with the Risk Management Progran (Accident Prevention Rule, EPA 1996a). 
2. All modeling assumptions and the rationale for their use should be clearly documented. The items that should be considered in this documentation are also identified in Section 6.2.2.

3. The capabilities of the dispersion model chosen to perform the analysis should be commensurate with the level of detail required in the analysis. This capability should be consistent with the "graded approach" which directs that "effort should he proportional to the complexity of the facility and the safety systems relied on to maintain an acceptable level of risk" (DOE-STD-1027-92).

4. The public domain dispersion models identified in Section 6.3 are conditionally recommended for use in SAR applications. In cases where alternative models may be needed for specific applications, the analyst should provide a clear and concise justification for model selection and application. It is strongly recommended that if alternative models are needed for use in consequence assessments for preparation of safety-basis documents, such models should have been subjected to performance evaluation and source code quality assurance $(Q / A)$ verification and validation. The code should also be readily available for public access and detailed scrutiny.

5. Use of hand-calculation procedures carried out with a scientific or programmable calculator is an appropriate and acceptable alternative to computer codes if adequate for the level of detail required in the analysis. Requirements for scoping evaluations (Level 1 Analysis Class, see Table 4.1 and discussion in Section 4.2.1), such as done to determine inventory limits or to conduct a preliminary hazard analysis, are at the appropriate level of detail for hand-calculation methods. Calculations performed in support of safety analyses may need to be supported by independent verification and review.

\subsubsection{Specific Guidance}

The guidance in this section is intended to provide analysts at DOE sites with a set of recommendations on conditions that should be considered in conducting consistent and scientifically sound DBI and BDBI consequence assessments. The information should be used in concert with the specific guidance given in the APAC Spills Working Group report (Brereton et al. 1996). For chemical consequence assessments, the BDBI should represent an accident scenario with the largest possible release quantity of the hazardous chemical of interest, considering administrative controls, 18 from a container/vessel or process line failure that results

18 Administrative controls are procedures that limit the quantity of a substance that can be stored or processed in a vessel or pipe at any one time, or procedures that occasionally allow a vessel or pipe to store larger than usual quantities (e.g., during shutdown) (EPA 1996a). 
in the greatest hazard zone or distance to the chemical-specific toxic endpoint. This quantity for single-phase gaseous or two-phase (gas plus liquid aerosol) releases should be assumed to have a duration of 10 minutes. A liquid spill should be assumed to be instantaneous and to spread to a depth of $1 \mathrm{~cm}$ in an undiked area or to instantaneously cover a diked area. Spilis onto porous soils or other surface substrates that would influence pool growth and heat transfer may be accounted for in the model applied in estimating the release rate due to pool evaporation (EPA 1996a).

The toxic endpoint or index is usually expressed as a concentration below which toxic effects would most likely not be observed within a one-hour exposure time. Background on chemical exposure concentration effects and toxic load is given in Section 2.4.3. The ERPG-2 or TEEL-2 toxic values or indices should be used in determining hazard zones (see recommendation in Section 6.2.2.3) for both the DBI and BDBI accident scenarios. Use of ERPG-2 values for consequence assessments is consistent with the intent of current EPA guidance for risk management plans (EPA 1996a), and the use of ERPGs in general and TEELs for chemicals without an assigned ERPG value is consistent with the recommendations from DOE's Subcommittee on Consequence Assessment and Protective Action (Craig 1996). These indices are provided, along with the definitions of the toxic level or health hazard they represent, in Appendix D.

The guidance provided in the following subsections is specific to modeling assumptions for BDBI and DBI consequence assessments. The guidance deals with worst-case and most likely, or alternative, assumptions for meteorological input (atmospheric stability, wind speed, temperature, relative humidity, and surface roughness), terrain and building influences, and the specification of receptor locations. Although specific worst-case parameter values are identified for certain release types, these values should be interpreted as reference points for key parameters used in determining the set of model input values leading to conservative consequence estimates. An iterative procedure is typically used in determining worst-case parameter values. An example of this procedure is given using the HGSYSTEM model in EPA guidance on the application of refined dispersion models for hazardous or toxic air releases (EPA 1993b). Modeling parameters used for the DBI or more likely scenarios should be representative of typical conditions at the accident site. The set of default parameters given in Table 6.1 is recommended as a starting point in conducting DBI assessments. The analyst, however, should attempt to obtain the most representative data available. Further guidance on conducting DBI and BDBI modeling assessments is given in the discussion that follows.

\subsubsection{Characterization of Meteorological Conditions}

Wind Speed and Atmospheric Stability - The proper specification of meteorological conditions, specifically wind speed and atmospheric stability class, is important in conducting DBI and BDBI (worst-case) impact assessments. Background information on the influence of wind speed and atmospheric stability on vapor cloud dispersion was given in Sections 2.2 .1 and 2.2.3. Some general observations on release types and stability-wind speed combinations that would lead to the required level of conservatism in BDBI vapor-cloud modeling are given in Table 6.2. 
TABLE 6.1 Suggested Typically Required Default Modeling Parameter Values ${ }^{\mathrm{a}}$ for DBI Assessments

\begin{tabular}{|c|c|c|c|c|}
\hline Parameter Name & Conditions & Remarks/Comments & $\begin{array}{c}\text { Recommended } \\
\text { Value } \\
\end{array}$ & $\begin{array}{l}\text { Units of } \\
\text { Measure }\end{array}$ \\
\hline Roughress Length & $\begin{array}{l}\text { Indusurial } \\
\text { Urban } \\
\text { Rural }\end{array}$ & $\begin{array}{l}\text { Large refinery/chemical plant complexes } \\
\text { "Built-up" area (See Table 6.3) } \\
\text { Agricultural areas, sparse trees and buildings }\end{array}$ & $\begin{array}{c}1 \\
0.1 \\
0.03\end{array}$ & $\mathrm{~m}$ \\
\hline PG Stability Class & $\begin{array}{l}\text { Unstable } \\
\text { Neutral } \\
\text { Stable }\end{array}$ & $\begin{array}{l}\text { Wartm/hot days, light winds with low cloud cover } \\
\text { Day or night wich cloud cover and/or high winds } \\
\text { Cold nights, clear sky, low wind, nural } \\
\text { Cold nights, clear sky, low wind, urban }\end{array}$ & $\begin{array}{l}\text { A } \\
D \\
\text { F } \\
\text { E }\end{array}$ & $\begin{array}{l}-{ }^{b} \\
- \\
-\end{array}$ \\
\hline Wind Speed & $\begin{array}{l}\text { Stability A } \\
\text { Stability D } \\
\text { Stabilicy F } \\
\text { Stability E }\end{array}$ & $\begin{array}{l}\text { Daytime, high insolation } \\
\text { Overcast, "high" winds } \\
\text { Cold nights, clear sky, low wind, nural } \\
\text { Cold nights, clęar sky, low wind, urban }\end{array}$ & $\begin{array}{l}2 \\
4.5 \\
1.5 \\
1.5\end{array}$ & $\begin{array}{l}\mathrm{m} / \mathrm{s} \\
\mathrm{m} / \mathrm{s} \\
\mathrm{m} / \mathrm{s} \\
\mathrm{m} / \mathrm{s}\end{array}$ \\
\hline Relative Humidity & - & Non-reactive fluids, no phase change & 50 & $\%$ \\
\hline Wind Direction & - & $\begin{array}{l}\text { Not a direct input parameter, chemical accident } \\
\text { consequence modeling; assume it always blows } \\
\text { towards the most sensitive area. }\end{array}$ & & \\
\hline \multicolumn{2}{|c|}{ Wind Speed Measurement Height } & NWS reference height & 10 & $\mathrm{~m}$ \\
\hline Receptor Height & & Ground Level & 2 & $\mathrm{~m}$ \\
\hline Averaging Time & & Based upon the toxicity index (e.g.. ERPG values) & 60 & 1 \\
\hline
\end{tabular}

a Other source parameters such as release rate and state, themodynamic and physical properties must be obtained or calcuiated according to the modeling application. Also sec Spills Working Group report (Brereton et al. 1996).

b $-=$ not applicable.

Source: Adapted from API (1996).

Dispersion and dilution are minimized under stable atmospheric conditions (Class F) with light winds, which would likely result in worst-case impacts from near-surface instantaneous or continuous releases of passive (i.e., neutrally buoyant) gases and continuous near-surface releases of dense vapors. On the other hand, worst-case consequences from puff or short-duration (i.e., $t_{d}<0.6 t_{t}$; see Section 2.4.5), near-surface releases of dense vapors are likely to be associated with neutral atmospheric stability (Class D) and moderate wind speeds. This distinction in worst-case dispersion behavior for dense puffs versus dense plumes and passive plumes/puffs is due to the fact that higher wind speeds tend to damp the lateral spread of shortduration dense-gas releases near the source, thus permitting downwind advection with less dilution (AIChE/CCPS 1996). This dampening for dense gas plume releases is not as significant due to a more sustained momentum driven turbulence for continuous releases, which lessens the influence of the tendency of surface-Iayer turbulence to damp internal spread. Slightly elevated 
TABLE 6.2 General Observations of the Most Likely Worst-Case Meteorology Influencing Dispersion and Release Rate for Various Release Types

Near Ground Level (e.g., vertical or horizontal jet)

$(\mathrm{h}=\mathrm{l}-10 \mathrm{~m})$

1. Passive puff or plume

2. Dense puff

3. Dense plume

Ground LeveI (e.g., pool evaporation) $(\mathrm{h}=0 \mathrm{~m})$
Stable with light winds

Neutral with moderate winds

Stable with light winds

Boiling pool $\left(\mathrm{T}_{\mathrm{bp}}<\mathrm{T}_{2}\right)$ : Surface heat transfer and chemicals" physical/thermodynamic properties plays dominant role. Nonboiling pool $\left(\mathrm{T}_{\mathrm{bp}}>\mathrm{T}_{\mathrm{a}}\right)$ : wind speed, stability, and ambient temperature are important in combination with the pool surface area, vapor pressure and mass transfer coefficient.

Elevated (e.g., roof vent, stack)

1. Passive puff or plume ( $50 \mathrm{~m}<\mathrm{h}<100 \mathrm{~m}$ )

Neutral conditions with moderate winds

2. Buoyant plume $(\mathrm{h}>100-200 \mathrm{~m})$

Very unstable, wind speed has a dual and compensating influence

a $h=$ effective release height (physical plus buoyancy and/or momentum components of rise).

b Refers to atmospheric stability class and wind speeds that result in worst-case consequences for a specific release type. For dispersion estimates, light and moderate wind speeds are typically defined in the tange of 1 to $2 \mathrm{~m} / \mathrm{s}$ and 3 to $4 \mathrm{~m} / \mathrm{s}$, respectively. As used hete, stable and neutral conditions are the Pasquill-Gifford, F and D classes, respectively. Very unstable is Pasquill-Gifford Class A. These variables have influences on dispersion andor release rate.

(10-50 m release height) dense puffs, if they sink to the ground within a few tens of meters of the release point, would, as instantaneous near-surface releases, tend to produce worst-case impacts under neutral and moderate winds.

Detemining worst-case conditions for pool evaporation is more complicated because of the many variables that influence both the rate of release from the pool and the dispersion of the vapor cloud once in the atmosphere. The evaporation rate from a cryogenic liquid spill (normal boiling point of liquid is less than the ambient temperature) depends almost entirely on the heat balance affecting the boiling liquid within the pool. Conduction from the surface is the initial and primary source of input heat transfer to the pool. This conductive heat transfer and the heat of 
vaporization of liquid are of primary importance in controlling the rate of evaporation from a cryogenic pool. Parameters that influence the conductive heat transfer and thus the evaporation rate include ambient temperature, pool surface area, and the thermal conductivity and diffusivity of the soil. Evaporation from non-boiling or -cryogenic (normal boiling point of liquid is greater than the ambient temperature) liquid pools is also heat-balance-dependent but is more influenced by atmospheric advection (i.e., convective heat transfer by surface winds) and solar radiation (i.e., radiative heat transfer from the atmosphere). These pools evaporate more slowly than cryogenic pools, depending on the atmospheric condition just mentioned and on the liquid's vapor pressure and surface area. Other parameters that play a role in controlling the evaporation rate include the molecular weight of the liquid, liquid-gas interface temperature (approximately equivalent to ambient temperature for slowly evaporating liquids), and the mass transfer coefficient, for which empirical relations have been determined (see also AIChE/CCPS 1996).

For passive elevated releases at heights of between 50 and $100 \mathrm{~m}$, regardless of release duration, worst-case off-site impacts are most likely associated with neutral stability and moderate wind speeds. Assessment of worst-case worker exposure may need to consider slightly to moderately unstable conditions. Worst-case impacts for strongly buoyant plumes with effective release heights greater than 100 to $200 \mathrm{~m}$ typically occur during moderate to very unstable atmospheric conditions. Wind speed has a dual effect on these releases by its contravening influence on downwind concentrations through its effect on plume rise and dilution.

Because of the complexities just described in establishing worst-case conditions, it is recommended that the general guidance given in Table 6.2 be used in determining the specific wind speed, stability, and/or ambient temperature (also see discussion below) combination that produces worst-case impacts. This determination should be done through an iterative process. This process is most needed for establishing the worst case for dense puffs, evaporating pools, and elevated releases. The worst case for near-surface passive puffs and plumes and longduration dense plume releases is typically assumed to be for wind speeds of $1.5 \mathrm{~m} / \mathrm{s}$ and atmospheric stability Class F. These conditions are what EPA recommends for worst-case accident scenarios regardless of the release type (EPA 1996a). However, as discussed above and as indicated in Table 6.1, these conditions will most likely not produce worst-case impacts for short-duration releases of dense gases.

Assessment of DBI or more-likely accident scenarios should use meteorological conditions that are most typical or representative of the facility location. These conditions should be identified by examining records of the frequency of occurrence of wind speeds and atmospheric stability classes over a sufficient period of time. If available, at least two years' worth of on-site meteorological data should be reviewed to determine the most typical wind speed and stability class conditions. If data from the nearest representative off-site National Weather Service station are to be used, at least five years' worth of records should be examined to determine appropriate meteorological conditions to be used for DBI consequerice assessments. Default or starting values are given in Table 6.1.

Temperature and Relative Humidity - Ambient temperature should also be considered in determining worst-case conditions for pool evaporation and, as already mentioned, 
for pool fires. For pool evaporation, the highest credible temperature (e.g., the 95th percentile of a five-year record of daily high temperatures for the warmest month of the year) should be used with iteratively determined wind speed and atmospheric stability for BDBIs. For pool fires, on the other hand, the lowest credible ambient temperature will typically induce the maximum rate of cooling in the plume and hence the largest rate of decline in plume elevation with downwind distance. As mentioned for pool evaporation, various combinations of wind speed and stability should be used in estimating pool fire BDBI impacts.

The water content of air is an important variable influencing heat transfer between the vapor cloud and air. Heat transfer generally enhances turbulence and dilution in the vapor cloud. When the humidity is relatively high, less air is required to mix with a cloud at a temperature different than that of ambient to achieve thermal equilibrium. This fact follows from the significantly higher heat capacity of water in comparison with that of dry air. Furthermore, a number of chemicals are hygroscopic and react with the moisture in air. These chemical transformations can dramatically change the physical-chemical behavior of the vapor release and should be considered when performing a BDBI or DBI analysis.

The rate and properties (e.g., temperature, relative humidity) of ambient air entrained into a vapor cloud influence the cloud's density and, therefore, its behavior (e.g., denser clouds will tend to spread more laterally because of gravitational forces). Before these effects are considered, the initial cloud density of a gaseous or aerosol release must be estimated. This parameter can be estimated by using the ideal gas law and by specifying the release temperature and the molecular weight of a single-component release or the average molecular weight of a multicomponent release. The molecular weight $\left(\mathrm{M}_{\mathrm{me}}\right)$ for mixtures can be determined by the mass fraction of each component as follows:

$$
M_{m c}=\sum_{i=1}^{n} \frac{m_{i}}{M_{i}}
$$

where

$$
\begin{aligned}
& m_{i}=\text { mass fraction of substance } i \\
& M_{i}=\text { molecular weight of substance } i, \text { and } \\
& n=\text { number of compounds in the mixture. }
\end{aligned}
$$

Surface Roughness - Objects or roughness elements on the ground surface, characterized in models with a surface roughness length or parameter $\left(z_{0}\right)$, affect the mean wind velocity and turbulence in the surface layer. This layer is generally defined as the lowest $50 \mathrm{~m}$ of the atmospheric boundary layer where frictional forces and horizontal shear stress are dominant. Although the most reliable estimates of $z_{0}$ can be obtained from measured wind profiles (see the Glossary [Chapter 7] to this report), estimates based on ground surface characteristics are lypically used. Another related and important parameter used in characterizing surface layer turbulence, typically used in the model reviews in this study, is the friction velocity $\left(u_{*}\right)$. This parameter is a direct measure of the frictional forces of wind and mechanical turbulence in the surface layer. Typical values of $z_{0}$ and $u_{*}$ for light and moderate wind speeds are given in Table 6.3. 
TABLE 6.3 Representative Surface Roughness and Friction Velocity Values in Constant Shear Stress Layer ${ }^{3}$

\begin{tabular}{|c|c|c|c|}
\hline \multirow[b]{2}{*}{ Surface Type } & \multirow[b]{2}{*}{$\mathrm{z}_{0}(\mathrm{~cm})$} & \multicolumn{2}{|c|}{ Friction Velocity, $\mathrm{u}_{*}(\mathrm{~cm} / \mathrm{s})$} \\
\hline & & $\begin{array}{c}\text { Light Winds, } \\
\overline{\mathrm{u}}=1.5 \mathrm{~m} / \mathrm{s}\end{array}$ & $\begin{array}{c}\text { Moderate Winds, } \\
\overline{\mathbf{u}}=4 \mathrm{~m} / \mathrm{s}\end{array}$ \\
\hline $\begin{array}{l}\text { Urban/suburban } \\
\text { Cities with very tall buildings }\end{array}$ & $\begin{array}{c}40-300 \\
300\end{array}$ & $\begin{array}{c}18.6-49.8 \\
49.8\end{array}$ & $\begin{array}{c}49.6-132.9 \\
132.9\end{array}$ \\
\hline Cities to large towns & 120 & 28.3 & 75.5 \\
\hline Small towns & 55 & 20.7 & 55.2 \\
\hline Town outskirts & 40 & 18.6 & $\quad 49.6$ \\
\hline $\begin{array}{l}\text { Woodlands and forests } \\
\text { Thin/thick grass up to } 50 \mathrm{~cm} \text { high }\end{array}$ & $\begin{array}{c}20-120 \\
5-10\end{array}$ & $\begin{array}{l}15.3-28.3 \\
11.3-13.0\end{array}$ & $\begin{array}{l}40.8-75.5 \\
30.1-34.7\end{array}$ \\
\hline Cut grass 1 to $5 \mathrm{~cm}$ high & $0.1-1$ & $6.5-8.7$ & $17.3-23.2$ \\
\hline Smooth sand & $10^{-2}$ & 5.2 & 13.9 \\
\hline Flat snow covered ground & $10^{-3}$ & 4.3 & 11.5 \\
\hline Very smooth (mud flats/ice) & $1 \times 10^{-3}-3 \times 10^{-3}$ & $4.3-4.7$ & $11.5-12.5$ \\
\hline
\end{tabular}

In general, the overall effect of increased surface roughness is enhancement of mixing between the vapor cloud and the environment as a result of surface-induced increase in turbulence. Occasionally, however, certain elevated (e.g., building vents) or surface releases may be affected by the orientation of adjacent surface elements (e.g., buildings) that can channel flow to produce elevated concentrations behind such elements (see discussion in Section 6.2.2.2). Most dispersion models can only accept a single value for surface roughness for a given set of calculations. The sensitivity nu for test scenario A-2 $\left(\mathrm{z}_{0}\right.$ increased an order of magnitude from $0.3 \mathrm{~m}$ to $3 \mathrm{~m}$ ) illustrates the reduction in centerline concentrations with increased surface roughness (see Figures 5.1 a and 5.3). The effect appears to be greater for the HGSYSTEM and SLAB models with distance from release $(100 \mathrm{~m}$ versus $1,000 \mathrm{~m})$. This result seems consistent with Britter and McQuaid (1988), who note that the influence of $z_{0}$ is less on dense-gas releases than on passive-gas releases. It is therefore recommended that for the conservative estimates required for the BDBI assessments and for consistency in performing these assessments, modeling should be conducted with $z_{0}$ and $u_{*}$ values consistent with "worst case" winds (e.g., see Table 6.3). Consequence assessments for the more likely or DBI release scenarios should use representative site-specific values. 
Finally, some care should be exercised when assigning a surface roughness value $z_{0}$ in the event of a vapor source located near ground surface. In many dispersion models, $z_{0}$ is the elevation at which the wind speed approaches zero. Therefore, if the roughness height exceeds the source height, molecular diffusion dominates the vapor transport modes in the vicinity of the source. Within this range, most dispersion models cannot provide a reliable estimate of vapor concentration. As the vertical elevation of the plume to the toxic endpoint of concen exceeds the height of the elements represented by $z_{0}$, the boundary layer approach to eddy turbulence again becomes valid. Most models, DEGADIS for example, use a linear interpolation for the wind speed for heights less than $2 \mathrm{z}_{0}$ so that the wind profile is continuous all the way down to the surface $(z=0)$. Thus, the $z \cong z_{0}$ dilemma in the logarithmic wind profile is avoided. Source heights for pipe ruptures and similar occurrences can easily be less than surface roughness typical of industrial complexes or urban areas $\left(\mathrm{z}_{0} \cong 0.5\right.$ to $\left.1 \mathrm{~m}\right)$.

\subsubsection{Characterization of Surrounding Terrain and Building Influence}

Flat-Plane Assumption Versus Complex Terrain - From a modeling standpoint, the simplest way to treat terrain is to characterize the region between the vapor source and receptor as an idealized perfectly reflecting flat plane. For this condition, there is assumed to be no turbulence in the flow field induced by features at ground level (i.e., the surface is assumed to be perfectly smooth), and any flux of air parcels impinging on the ground surface will be perfectly reflected back into the air stream with no loss (i.e., deposition) of any tracer species present in the moving air mass. This characterization choice is especially advantageous from the perspective of selecting the other worst-case parameters, such as for elevation of the source or meteorological conditions, for instance. With the flat-plane assumption, it is then possible to prescribe generic, unqualified assigninents of values for the other parameters that will ensure worst-case impact (see recommendation on surface roughness parameter in Section 6.2.2.1). In applications where surrounding terrain relief is nearby (within 2 to $5 \mathrm{~km}$ ) and is significantly elevated ( 210 times the height of a near-surface release), models that account for complex terrain influences should be used in conducting DBI assessments. The flat-plane assumption does not ensure worst-case results in a situation where a heavier-than-air vapor source is located in a valley or large depression or flow is channeled by the pressure of buildings or natural features. In such cases, a complex terrain or building wake effects model should be considered for use in performing the vapor dispersion analysis.

Finally, in applications where topographic features are large compared with the scale of the release, use of complex terrain or building wake models should also be considered. Guidance for the determination of when topography should be treated on a detailed local scale is given, for instance, in Britter and McQuaid (1988) and Britter (1982).

Presence of Buildings or Obstacles to Flow - In the presence of obstacles to flow, vapor concentration fields can become highly complex (see Section 2.1.7). With the exception of special building- (or terrain-) source configurations, the spatially average vapor concentration is generally not increased above the values that would be present in the absence of obstacles to flow. However, the concentration isopleths in almost all cases are distorted in such a manner that a receptor may be exposed to a higher concentration because of a shifting of the areas enclosed by a given concentration isopleth. 
The most recent reviews of methods to estimate the effects of structures on toxic vapor dispersion can be found in Schulman et al. (1990), Britter (1989a), Brighton (1989), and Wilson (1979). Nearly all the experiments conducted on the effects of building aerodynamics and the formulas for estimating toxic gas concentrations resulting from these effects are most valid for passive releases. Relatively little information is available for dense-gas dispersion around buildings. Formulas are available for estimating the effects of building canyons on plume confinement, concentration on building faces due to roof or side vent releases, and near-wake or cavity concentrations due to roof vent releases. These methods are summarized in recent AIChE/CCPS guidelines (1996) and are incorporated in the HGSYSTEM model (version 3.0). Far-wake estimates can be made with EPA's Industrial Source Complex (ISC) Model (EPA 1995). These building cavity and wake influence approaches are adequate for both BDBI and DBI consequence assessments.

Accurate estimates of the vapor concentration field near complex structures or terrain features require either site-specific experimental data (wind tunnel and/or field measurements) or use of detailed computational fluid dynamics codes. One disadvantage of the latter approach is that use of such codes requires imposition of complicated boundary conditions and specification of detailed turbulence parameters in order to achieve accurate results.

\subsubsection{Characterization of Receptors of Interest and Identification of the Haxard Zone}

Near-Field Versus Far-Field Dispersion Prognosis - Many dispersion analysts maintain that the commonly used dispersion models (e.g., Gaussian plume and puff equations) are not valid in the near field (i.e,, distances less than 100 to $200 \mathrm{~m}$ from the source). If one accepts the precept of self-similarity in dispersion analysis, then it is just as valid to assume that the Gaussian plume equation applies immediately at the source and hundreds of kilometers downwind, as well as in the typically quoted range of $100 \mathrm{~m}$ to $50 \mathrm{~km}$ (tracer experiments supporting the Pasquill-Gifford dispersion parameters ranged from 50 to $800 \mathrm{~m}$ and were extrapolated to $100 \mathrm{~km}$ ). The problem lies in the question of whether the parameters or functional relationships are adequately represented in these ranges. A brief discussion of the importance of averaging time considerations is given in Section 2.4.2. A more detailed discussion is provided in AIChE/CCPS (1995).

Although many instances require the application of more detailed computer models for simulation of near-field dispersion, in some cases fewer details are more appropriate. Instances where more complex models would be more appropriate include situations where complex cloud dynamics (e.g., density and temperature gradients, eddy turbulence occurring over multiple scales, heat and momentum transfer effects) may dominate the transport and dispersion of a vapor cloud. As downwind distance increases, details of the source term (e.g., source dimensions, release thermodynamics, variability in vapor release rate, release elevation above ground) become correspondingly less important to the predicted vapor concentration exposure at the receptor location.

Identification of the location of maximally exposed receptors for accident scenarios involving passive-gas releases is generally amenable to hand or calculator methods described, for 
example, in Tumer's (1994) Workbook of Amospheric Dispersion Estimates. Dense-gas calculations can be performed with the aid of nomograms in Britter and McQuaid's (1988) Workbook on Dispersion of Dense Gases (also see AIChE/CCPS 1996). Worst-case consequences can be expressed as those conditions that lead to the largest distances downwind for a given concentration (e.g., toxicity value expressed in terms of an ERPG or TEEL value) or that lead to the largest area for that given concentration value. The conditions leading to the largest distance are not necessarily the same as the conditions leading to the largest area. Most models reviewed in this report provide a capability for estimating a hazard zone or area that is specific to the toxicity value of the chemical compound of interest. For consistency, both the BDBI and the DBI hazard zone or largest area should be estimated for consequence assessments on the basis of the guidance provided in this section.

Elevation of the Receptor with Respect to the Source - The maximum vapor exposure to an individual on an idealized perfectly reflecting flat-plane surface with no obstacles to flow would occur when the vapor source was located at the same elevation as the receptor's breathing zone. For this reason, the vapor source is often assumed to be located at or near ground level, unless some feature of the process or postulated accident renders this situation highly unlikely. However, as described in Section 6.2.2.2, locating the source at ground level may also increase interaction of the vapor cloud with the ground substrate and any associated terrain features or obstacles to flow. Elevated releases may in a very few cases increase the downwind distance to a selected level of concern, because an elevated plume may not be subject to the aerodynamic effects of turbulence-enhancing terrain features between a source and receptor that would otherwise reduce vapor concentration and mitigate the impact of a ground-level release.

\subsection{CHEMICAL CONSEQUENCE ASSESSMENT MODEL RECOMMENDATIONS}

The selection of a particular model or type of modeling procedure for use in chemical consequence assessment should be justified and documented in terms of the objectives and the constraining conditions of the analysis at hand. This process should include consideration of criteria for the omission or inclusion of factors that may determine the most appropriate type of model or analysis procedure for use. It may be necessary to consider, for example, the omission or inclusion of such factors as dense-gas dispersion behavior; the influence of topography, surface conditions, and the presence of buildings on dispersion and source behavior; the influence of coastal meteorology; and the characterization of surface features as typically urban, suburban, industrial, rural, or some variation. Conclusions on the suitability of a model will need to be related to the specific characteristics of the release and of the site of interest. The fact that a particular dispersion or transport mechanism is not addressed by a model that is available, or that the mechanism would be difficult or expensive to address, should not be regarded as adequate criteria for excluding that mechanism from consideration if there is a case for its inclusion on technical grounds. The guiding principle in the justification of the procedures chosen should be the demonstration of fitness for purpose.

Although in many cases there will be a need to use computer software implementations of models in assessments, there may also be instances when scoping or screening hand or calculator methods are adequate (see Section 6.2). For example, when such calculations reliably show that the upper limit of a numerical quantity is so far below an appropriate reference level of 
concern that more detailed estimation is not merited, it would be a waste of resources to apply more involved methods.

The Tier I model recommendations of the CDCA Working Group are summarized in Table 6.4. A factor considered in formulating these recommendations included the need to apply a graded modeling approach in chemical dispersion modeling in support of SAR, BIO, and JCO safety basis documents (SBD). In addition, the working group carefully weighed its review and evaluation of each of the Tier I and II codes, including the strengths and weaknesses and the capabilities and attributes of each model, as well as the insights gained from the Tier I test scenarios. Use of those scenarios should not be considered either a substitute for model performance evaluation with field observations or a means for determining which model is "best." However, comparison of results from models run for the same test case does provide a measure of the relative variability in model estimates through observation of the specific release scenario conditions for which the code yields comparable or outlier results. This comparison was helpful in developing the best practice guidance on design and beyond design basis (i.e., worstcase) accidents presented in Section 6.2 and also in formulating the model recommendations given in this section. It should be noted that the CDCA Working Group is not presenting specific recommendations on the basis of the relative hazard class for a particular DOE nonradiological facility. Working group members felt that we could not formulate such recommendations in an objective and scientifically defensible manner without further consideration of the specifications or details used to support facility hazard classifications. Another important factor regarding the recommendations was consideration of the user friendliness of the codes. A few codes classed as conditionally recommended (Table 6.4) are identified in the discussion below as requiring a relatively large computer and human resource expense in problem setup, code execution, and result interpretation. Since two of these codes, specifically FEM3C and HOTMAC/RAPTAD, have unique attributes or capabilities that would serve special-purpose analysis needs, they are

TABLE 6.4 Summary of Tier I Model Recommendations ${ }^{a}$

\begin{tabular}{|c|c|c|c|}
\hline $\begin{array}{c}\text { Conditionally } \\
\text { Recommended for } \\
\text { SBD } \\
\end{array}$ & $\begin{array}{c}\text { Conditionally } \\
\text { Recornmended for } \\
\text { Special/Limited SBD }\end{array}$ & $\begin{array}{c}\text { Conditionally } \\
\text { Recommended for further } \\
\text { Review and Evaluation } \\
\text { for SBD }\end{array}$ & $\begin{array}{l}\text { Not Recommended } \\
\text { for } \mathrm{SBD}\end{array}$ \\
\hline $\begin{array}{l}\text { ALOHA } \\
\text { DEGADIS } \\
\text { HGSYSTEM } \\
\text { SLAB }\end{array}$ & $\begin{array}{l}\text { ADAM } \\
\text { CALPUFF } \\
\text { FEMSC } \\
\text { INPUFF } \\
\text { TSCREEN }\end{array}$ & $\begin{array}{l}\text { CASRAM-SC' } \\
\text { HOTMAC/RAPTAD } \\
\text { SCIPUFF }^{b}\end{array}$ & VLSTRACK \\
\hline
\end{tabular}

a SBD = Application to chemical accident consequence assessment for safety basis documentation; codes in bold face italics have dense-gas capabilities.

b New or evolving versions of these codes may warrant further consideration and review by APAC or succeeding program. Except for CASRAM, these models have been published in the peer-reviewed literarure and are well known in the air dispersion modeling communiry. SCIPUFF and HOTMAC/RAPTAD (proprietary version) are established, widely used models whose latest versions are still in the process of being tested and/or further developed. 
included among the models conditionally recommended and recommended for further review, respectively.

Finally, as discussed in more detail below, the committee recommendations are "conditional" in that the recommendation to use a particular code applies only for that code's capability domain, as identified in Table 5.2 .

\subsubsection{Tier I Models}

\subsubsection{Recommendations}

As shown in Table 6.4, the CDCA Working Group placed each of the Tier I models it reviewed into one of four groups: (1) conditionally recommended, (2) conditionally recommended for special/limited applications, (3) conditionally recommended for further review and evaluation, and (4) not recommended. In general, a model was conditionally recommended if it has been extensively applied in chemical accident consequence assessment or emergency planning applications and the working group felt the model could have a sufficiently broad application to potential facility accidents involving chemical releases in support of DOE safety basis documents (SBD). The summary tabulations from the model reviews presented in Chapter 5 of this report (Table 5.3) played a significant role when the working group judged the breadth of applications for the conditionally recommended models. Codes in this group were also required to have previously gone through performance evaluation with field and/or Iaboratory data and/or to compare reasonably well with inodels subjected to such evaluations (see the Chapter 5 model comparisons and the individual model reviews below). All of the conditionally recommended models are capable of handling accidents involving dense vapor clouds. These types of potential accidents were judged by the working group to be most predominant within the DOE complex.

The five Tier I codes that were conditionally recommended for special/limited applications generally have special or limited applications that are less broad than those of the conditionally recommended models. Although in theory one of these, the FEM $3 \mathrm{C}$ code, is potentially applicable for wider use, it was conditionally recommended only for special/limited applications because of practical considerations. Use of the code is both human and computer resource intensive, requiring the use of a Cray "super computer" platform for execution. This limiting requirement weighed heavily in the working group's recommendation. ADAM and TSCREEN were conditionally recommended for special or limited application primarily because of their limited chemical database and a screening level design, respectively. Two of the other models (CALPUFF and INPUFF) that were conditionally recommended for special or limited applications do not have the capability to address dense vapor clouds.

Three Tier I models were conditionally recommended for further review and evaluation. Two of these, SCIPUFF and CASRAM-SC, were in "beta" version status and lacked complete documentation when reviewed. However, both of these codes have special attributes and potentially have sufficiently broad applications to warrant a further review when official versions (e.g., 1.0 or higher) are released and complete documentation becomes available. The other model in this group, a public domain version of HOTMAC/RAPTAD, also was not fully 
documented and is still evolving. The working group's reviews indicated that although these models have technical merit, they are not yet ready for broad applications in support of chemical consequence analysis for DOE facilities.

One model was designated as not recommended for DOE safety analysis applications. The VLSTRACK model was not recommended because it was developed for rather specialized military applications that limit its suitability for analyzing chemical accidents at DOE facilities.

\subsubsection{Supplemental Assistance to Analysts}

As a supplemental aid to the recommendations contained in this report, the CDCA Working Group developed a framework to help potential model users identify appropriate models for specific applications. This framework, as illustrated in Figure 6.1, involves a "decision tree" approach that can be used in the model-selection process. We have included only a few of the many attributes or features that chemical dispersion models can include (see Table 5.2). Therefore, this figure serves as an example of an approach that could be expanded and refined for future use by safety analysts'or DOE personnel responsible for review of SBD.

To use the decision tree in Figure 6.1, one starts at the top level and works down the tree, successively answering the question in each diamond-shaped box. If the model feature in focus is required for the chemical release being evaluated, then the "yes" branch should be followed. If the feature is not required, then the "no" branch is taken. This process leads to the identification (at the end of the last branch) of the model or models appropriate for the situation being evaluated.

\subsubsection{Rationale for Recommendations}

In addition to the considerations discussed above, other rationale for the CDCA Working Group's recommendations for each Tier I model are summarized below. The primary basis for each recommendation is summarized first, followed by brief discussions of (1) code history (2) documentation and ease of use, (3) application strengths and weaknesses, (4) test result comparisons, and (5) performance as measured by comparison with field data. The models are discussed in alphabetical order.

It should be noted that the pertinent "condition" for all conditional recommendations is that a model is recommended for use only within its applicable capability domain(s), as identified in Table 5.2 and indicated in Figure 6.1. The working group discussed but made no attempt to rank or rate the models on the specific attributes of the models reviewed. It was judged that there was no objective way to do this without a comprehensive model performance evaluation and sensitivity analysis. Another condition considered by the working group was to limit model use to applications within its performance domain. This idea was rejected, however, because currently only limited data are available for use in establishing such domains. Perhaps the recommendations for future model performance evaluations, given in Section 6.4, would permit refinements to this guidance and allow specification of performance domain requirements. 
ADAM (Air Force Dispersion Assessment Model) _- Version 2.1

\section{Conditionally recommended for special SBD applications}

ADAM is conditionally recommended only for special SBD applications (within its application domain) primarily because its limited library includes only eight chemicals, two (phosgene and nitrogen tetroxide) of which have very specialized weapons and rocket propellant applications of special interest to the Air Force.

ADAM was originally developed in the mid-1980s to assist the Air Force in assessing possible hazards from potential accidents involving use of rocket propellants and oxidizers at various Air Force installations. The model was upgraded in 1992 to address larger surface roughnesses and to correct noted errors in the coding of some equations. The upgrade also included the addition of two chemicals, fluorine and hydrogen fluoride, to its original library of six chemicals (ammonia, nitrogen tetroxide, sulfur dioxide, phosgene, hydrogen sulfide, and chlorine). The model's technical documentation and user's guide are well written and complete (reflecting upgrades to the code). ADAM's, major strengths are that it treats a wide variety of source release conditions, and it accounts for dense-gas effects, as well as source thermodynamics effects, latent heat exchanges, phase changes, and near-source in-cloud chemistry. In the limit of passive or neutrally buoyant gases, ADAM asymptotically approaches the Air Force's AFTOX model.

The code's weaknesses are that it can only be applied for the eight chemicals in its chemical library and cannot be applied to elevated releases or vertically directed jets. Model predictions for the release scenarios used in this study revealed that ADAM sometimes predicts lower concentrations than the other Tier I models. However, in some test cases, the results from ADAM are in the middle of the range of predictions by the other models. Hanna et al. (1991) have shown on the basis of four experiments from the Desert Tortoise field data that ADAM underestimates observations by a factor of about 2 to 3 at distances of $100 \mathrm{~m}$ from the release. At distances of about $3 \mathrm{~km}$, the results are mixed, with underpredictions of a factor of 3 or 4 for two experiments and overpredictions for two experiments. The model developers have shown good agreement between the ADAM predictions and observations of temperature and concentration at the Goldfish HF field trials.

\section{ALOHA (Areal Locations of Hazardous Atmospheres) - Version 5.2}

\section{Conditionally recommended for $S B D$ applications}

ALOHA is conditionally recommended for SBD applications within the model's capability domains. This recommendation is based primarily on its broad acceptance and application in regulatory and emergency response situations and its ability to provide concentration estimates from both dense- and passive-plume behavior that are within a factor of 2 of field observations.

ALOHA is actively maintained, with upgrades released about every two years. The model was first written in the early 1980 s as a passive gas plume model for in-house response. A chemical property library, meteorological station port interface, and base mapping 


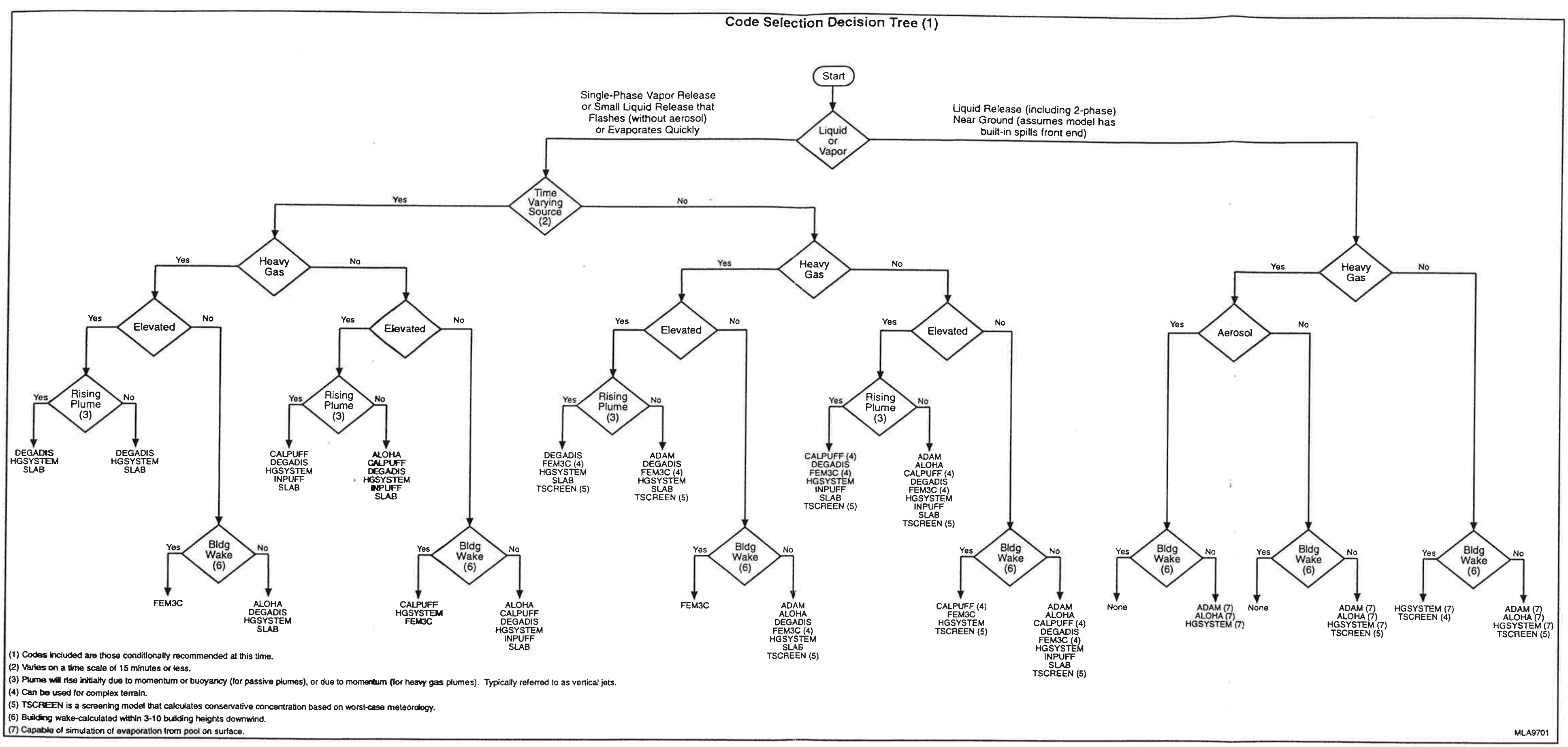

FIGURE 6.1 Example of "Decision-Tree" Approach to Selecting Appropriate Dispersion Models 
capabilities were added in the late 1980s. Time-dependent Gaussian and heavy-gas dispersion algorithms, along with some additional source strength capabilities, were added in 1991. A Microsoft Windows version was released in 1992, with a plan for release of version 5.2.1 sometime in early 1997. Although the theoretical description of ALOHA (version 5.0) has not been updated since 1992, the changes in the code since version 5.0 are reflected in the model's users guide (EPA/NOAA 1995) and in a newsletter periodically issued by the National Safety Council. The code has a graphical user interface (GUD) designed primarily for ease of use by "first responders" (such as community fire departments). It contains pull down menus with prompts and warning and caution messages. The model takes only about 15 minutes to set up and has a rapid execution time (with fast CPU, typically less than 1 minute). ALOHA's major strengths are its user-friendly dense- gas capabilities and its extensive library of chemicalphysical property data used in characterizing source physics and thermodynamics. The model is also able to address a wide variety of releases from pipe and tank ruptures, including two-phased flows involving pressurized or cryogenic releases.

ALOHA's major weaknesses are its inability to account for the elevation of the release of dense gases (it assumes release is at ground level) and to account for variable averaging times, which may be important in obtaining accurate assessments of exposure and health consequences. Although not a problem for all uses, its GUI may not be well suited to consequence assessment applications requiring many iterations (e.g., a production run involving assessment of many accident scenarios).

For the test cases, the concentration predictions of ALOHA were within a factor of 2 of the predictions of the three other dense-gas codes conditionally recommended for SBD in this study (DEGADIS, HGSYSTEM, and SLAB) (see discussion in Section 5.2.2). Of particular note is the conclusion that the predictions of ALOHA, DEGADIS, HGSYSTEM, and SLAB all appeared to agree within a factor of 5 (on average). In a separate quality assurance study (Evans 1994), the National Oceanic and Atmospheric Administration has compared ALOHA results with concentrations from the Goldfish field experimental data. ALOHA has met the Gaussian model limitation for flat terrain of a factor of 2 accuracy for a wide variety of release scenarios. The working group members and the model developer are not aware of any independent performance evaluations for the current version of ALOHA. (Hanna et al. [1991] evaluated an early non-dense-gas version [4.2] of the model.)

\section{CALPUFF - Version 3.0}

\section{Conditionally recommended for special SBD applications}

CALPUFF is conditionally recommended for special SBD applications within the model's capability domain primarily because of its design for passive, continuous-release plume dispersion applications. The model is not capable of treating dense-gas dispersion.

CALPUFF was originally developed for the California Air Resources Board by Sigma Research. The code is part of a modeling system that includes both pre- and postprocessors, including a three-dimensional meteorological model. The model is being distributed by the EPA 
for general testing and review, and approval for its use for regulatory assessments should be sought on a case-by-case basis. The code documentation is thorough and easily attainable.

CALPUFF is well documented and has a user-friendly interface. The code is run in a Windows environment, as are the postprocessors. The calculations can include time- and spacevarying parameters. The variety of input variables and options available make the code very flexible, but input requirements are somewhat cumbersome. Output is detailed; it may actually provide more information than necessary for most applications. Time scales are a minimum of one hour and, thus, are longer than needed for most chemical accident analyses. It has been reported that version 6 (completed after this study began) contains provisions for time steps of less that one hour.

The most significant weakness of CALPUFF, from the DOE perspective, probably is the lack of a front end spills model that computes evaporation, jet effects, etc. The model does not compute the area of impact above ERPG level, nor does it have a dense-gas model. Two of the seven test problems used in this evaluation could not be run with CALPUFF. The results of the problems that could be run were most like the results for INPUFF and ALOHA. The largest difference between CALPUFF and other models in the study was for the elevated release (test case D). For these cases, CALPUFF generally predicted lower concentrations near the source because it could not handle dense gases.

In an evaluation by the Interagency Workgroup on Air Quality Modeling (TWAQM), CALPUFF results were compared with tracer data collected during the Cross-Appalachian Tracer Experiment (CAPTEX). (EPA 1995). As a result of that evaluation, the model has been enhanced to make it more suitable for mesoscale applications.

CASRAM-SC (Chemical Accident Stochastic Risk Assessment Model - Single Case) Version 0.8

\section{Conditionally recommended for further review and evaluation}

CASRAM-SC is conditionally recommended for further review and evaluation in the future because the model is still in the preliminary draft stage of development, and the version available for review had neither a users guide nor complete technical documentation. Further consideration for future review and evaluation should be given only when this documentation is in final form. Publication in a peer reviewed joumal would aid a decision for further model review. The model's features and attributes could eventually have special (e.g., risk quantification), but limited, utility in SBD applications. The possibility of broader SBD applications could be considered in future reviews if the model's planned upgrade includes the ability to treat dense-gas dispersion.

The strengths of CASRAM are that it can calculate source terms, and it includes a sophisticated module for accounting for atmospheric boundary layer wind, temperature, and turbulence profiles. However, the code does not treat dense gases and assumes that all releases are at ground level. In our test-case comparisons, the model predictions appeared to be very sensitive to some meteorological parameters, such as solar radiation. A users guide would have 
been of value in the seiection of appropriate input parameters (i.e., develofers guidance on choice of meteorological parameters such as solar angle. cloud cover, and wind speed) in running cases for a particular Pasquill-Gifford stability class. The model has not bean subjected to an independent model geriormance avaluation.

\section{DEG.tDIS (Dense Gas Dispersion) - Version 2.1}

\section{Conditionally recommended for $S B D$ applications}

DEGADIS is conditionally recommended for SBD applications within the model's capability domains primarily because of its wide acceptance and application in regulatory and other situations and its ability to provide concentration estimates from both dense- and passiveplume behavior that are wichin a factor of 2 of field observations. The model is also listed as an EPA "Appendix B" refined air quality model that may be considered for individual regulatory applications on a case-by-case basis (Guideline for Air Quality Modeling, EPA 1996b).

DEGADIS (Yersion 1.0) was developed for the U.S. Coast Guard and Gas Research Institute in 1985. In 1988, Havens (1988) interfaced Ooms' (1974) jet model with DEGADIS to create version 2.0; in 1989, Spicer and Havens (1989) modified Ooms' jet model to create DEGADIS version 2.1. ${ }^{19}$ The model is accessible through the EPA's SCRAM Bulletin Board (BBS/TTN 2000, Web page access at: http://www.epa.gov/scram001). Although the model is fairly easy to run, the user must take special care to fully understanding the user options and the data input requirements (e.g., distinction between isothermal and nonisothermal simulations, release density considerations in specifying initial and final mole fractions). The users guide alone is not adequate in explaining how to set up release scenarios in a manner that will lead to results that are consistent and within the demonstrated performance range of the model. The user is strongly encouraged to supplement the users guide with information from such references as the EPA's Guidance on the Application of Refined Dispersion Wodels for Hazardous/Toxic Air Releases (EPA 19936).

A major strength of the model is its ability to address transport and dispersion of many types of dense-gas releases and to account for a variety of surface roughness elements. It can also address atmospheric dispersion of contaminant releases in passive dispersion flow regimes. A major weakness is the lack of a source release model or a spills front end. Another weakness is its inability to account for the effects of buoyant plumes. DEGADIS can only address pure chemical releases, not chemical mixtures or chemical transformations.

In the test runs for this study, DEGADIS predicted concentrations within a factor of 5 (on average) of the model predictions of the other dense-gas codes that are conditionally recommended for SBD in this study (ALOHA. HGSYSTEM, and SLAB) (see discussion in Section 5.2.2). DEG.ADIS sometimes predicts relatively high near the source for the ambient temperature chlorine scenario, but performed as expected for the other scenarios. Of particular

19 A new version of DEGADIS is expected to be ready for release in early 1998. Additional details are available through Steve Wiersma at the GRI. 
note is the conclusion that for the short-duration $\mathrm{HF}$ gas/aerosol mixture scenario, the predictions of ALOHA, DEGADIS, HGSYSTEM, and SLAB all appeared to agree within a factor of 2 for distances greater than $1,000 \mathrm{~m}$. When compared with the Desert Tortoise and Goldfish field data, DEGADIS predictions (corrected for initial dilution effects from aerosol jets) demonstrate reasonable performance, with a mean bias of about $30 \%$ and typical uncertainty or variance in the range of $50 \%$ to $70 \%$ (Hanna et al. 1991).

FEM3C (3-Dimensional Finite Element Method) - Version C

\section{Conditionally recommended for special SBD applications}

FEM3C is conditionally recommended only for special SBD applications within the model's capability domain primarily because of its resource-intensive (both computer and user) requirements. However, the model is very capable of treating ground or elevated releases of dense gases and has special capabilities for treating atypical plume behavior (i.e., building wake effects) and flow fields in complex terrain.

FEM3 was first released in 1983 as a modified Galerkin finite element method for solving the time-dependent conservation equations of mass, momentum, energy, and species of an inert gas. Subsequent upgraded versions of the code were released in 1988, 1990, and the final version in 1994. At present, no technical support is provided for the code and no further enhancements are planned. The reference technical manual and users guide are complete and provide all the necessary information to set up and run the code. Although the code assumes all physical quantities are specified in MKS units, it would useful if the users guide explicitly defined the units for each input item. The user documentation would also be more helpful if a companion tutorial document were available, especially to assist the analyst in constructing the computational grid and specifying the boundary conditions. The users guide currently lists the input requirements for use of several grid generation options, but only refers the user to select textbooks for further information, such as definition of tems and the actual implementation of the grid structure.

FEM3C possesses a number of outstanding strengths, such as (1) capability to model complex turbulent fluid flow in the presence of complex terain and obstacles to flow, (2) accommodation of multiple, simultaneous sources, and (3) capability to tailor the meteorological conditions and wind flow field to satisfy a variety of boundary conditions, thus giving the code the flexibility to model very site-specific release scenarios. The weaknesses in the code include the following: (1) it cannot accept a number of common vapor emission source terms (e.g-, pressurized jets, timle-varying vapor emissions), (2) the aerosol model is incomplete in the sense that it does not model all the relevant physical behavior (e.g., droplet evaporation, rainout), (3) the code is configured specifically for execution on the Cray (a parallel-processing computer), and, therefore, porting the code to another mainframe architecture or serial computing hardware would likely require a large programming effort, and (4) the output requires significant postprocessing by the user to obtain concentration values at specific locations and for specific averaging times. 
Because the amount of setup time required for each test problem was substantial and because only limited Cray time could be secured for this analysis, the probiem evaluations focused more heavily on evaluating the code functionality, capabilities, and reasonableness of the final results. ${ }^{20}$ Large-scale field data validations of the code have been published for the Buro series LNG spill tests, the Thorney Island experiments, and the LNG vapor bartier verification field trials, also known as the Falcon Series. FEM3C code performance in comparison with the Burro data was extremely good in virtually all test cases. FEM3C also predicted within a factor of 2 the vapor concentration associated with dispersion from a fenced storage area for the Falcon data. The model consistently underpredicted the vapor concentration in the Thomey Island trials by as much as a factor of 2 . The causes for the underpredictions resided in the inability of the turbulence model present in the first version of FEM3 to handle the early phase of a instantaneous complex flow problem, subsequently leading to the $k$ - $\varepsilon$ turbulence model present in FEM3C.

\section{HGSYSTEM (Heavy Gas System) - Version 3.0}

\section{Conditionally recommended for SBD applications}

HGSYSTEM is conditionally recommended for SBD applications within the model's capability domains primarily because of its wide acceptance and application in regulatory and other situations. In independent model performance evaluations, the code has shown its ability to provide concentration estimates, from both dense- and passive-plume behavior, that are within a factor of 2 of field observations. The model is also listed as an EPA "Appendix B" refined air quality model that may be considered for individual regulatory applications on a case-by-case basis (Guideline for Air Quality Modeling, EPA 1996b).

The first public domain version of HGSYSTEM (version 1.0) was released in November 1990. The current version (3.0), which is the model's second public domain release, was released in 1994. A uranium hexafluoride $\left(\mathrm{UF}_{6}\right)$ version of the code, HGSYSTEM/UF 6 , was released in 1996. This recent history indicates that the code is actively maintained by the developer. The technical reference manual and users guide are sufficiently clear and detailed to provide the user with the necessary background to set up a problem. Additional guidance, perhaps with sample problems, in the users guide would be useful in interpreting the numerical results.

The major strengths of the code include (1) efficient handling of time-dependent dispersion by automated selection of advection and averaging time, (2) treatment of multicomponent mixtures and vapor-aerosol generation, (3) detailed treatment of $\mathrm{HF}$ and $\mathrm{UF}_{6}$ source physics and thermodynamics and dispersion, and (4) a favorable independent model performance evaluation (Hanna et al. 1993). A weakness of the code, as noted in the execution of the test problems for this evaluation, is that under very stable conditions with low wind speeds for evaporative simulations and under low exit velocity for stack releases, the model exhibits

20 A workstation version of FEM3C is now available for limited distribution. Further details are available through Steve Wiersma at the GRI. 
some compurational instabilities. It should also be noted that the choice of the most appropriate modeling approach does require a fair amount of prior user experience and familiarity with the code's features. This observation stems from the numerous altemative options available for modeling a given release scenario. A few bugs in the code have been noted in the evaluation and passed along to the developer.

In general, the model combines relative simplicity of input with advanced modeling capabilities to yield a widely applicable code that would support most SAR chemical release scenario analyses. In the test runs for this evaluation, HGSYSTEM predicted concentrations within a factor of 5 (on average) of the predictions of ALOHA, DEGADIS, and SLAB (the three other dense-gas codes conditionally recommended for SBD in this study). Model performance, when compared with field observations, showed that HGSYSTEM (version 1.1) performed well (a relative mean bias of about $+30 \%$ and relative scatter about equal to the mean) in comparison with the performance of six other public domain and proprietary codes. Its predictions are as good as three of the four best models that are clustered together on the model performance graphs. All of the models tended to overpredict plume widths and underpredict plume depths by about a factor of 2 (AIChE/CCPS 1996).

HOTMAC/RAPTAD (Higher Order Turbulence Model for Atmospheric Circulation/Random Particle Transport and Diffusion) - Version January 1996

\section{Conditionally recommended for further review and evaluation}

HOTMAC/RAPTAD is conditionally recommended for further review and evaluation primarily because the version available for review did not have adequate user documentation for applying the code in a consistent and reliable manner. The model's features and attributes could eventually have special, but limited, utility in SBD applications (e.g., for prognostic wind fields in complex terrain). Possible use for broader SBD applications would be considered in future reviews if the model's planned upgrade includes the capability to treat dense-gas dispersion. The private sector version of the model is also listed as an EPA "Appendix B" refined air quality model that may be considered for individual regulatory applications on a case-by-case basis (Guideline for Air Quality Modeling, EPA 1996b).

The inception of HOTMAC began in 1982 with work by Yamada (1988), but the first actual full-scale version of the code was released in 1987. The particle transport code RAPTAD, designed to be used in conjunction with the prognostic meteorological model HOTMAC, was first described in the public literature in 1990 . The code has been upgraded since then; in fact, at least one revised version of the code was released following the January 1996 version evaluated in this study. A computer interface between the GASFLOW in-facility (indoor) transport and dispersion model (Spore et al. 1996) and HOTMAC/RAPTAD is under development at Los Alamos National Laboratory (LANL). Work is currently being conducted at LANL to complete a users guide for the code. The reference technical manual has been completed to a greater detail than the users guide, but stili requires updating to reflect some of the new modeling capabilities incorporated into the code. Many code features and input requirements may be unfamiliar to the safety analyst accustomed to modeling only relatively small, episodic releases. To make the code more usable by the safety analysis community, it would be very advantageous to develop a 
companion tutorial document, especially to assist the analyst in constructing the computational grid and in specifying the boundary conditions, and to provide some guidance of when nudging (Newtonian relaxation) is required, as well as some practical examples.

The primary strengths of HOTMAC/RAPTAD are (1) its modeling capabilities in the areas of multiple sources, complex air flow fields, and the effects of complex terrain on wind, temperature, and turbulence fields; (2) its use of prognostic equations for both the turbulent kinetic energy and length scale to drive vertical eddy diffusivity; (3) its relative numerically stable and computationally efficiency for codes of similar genre; and (4) its ability to allow for multiple (i.e., large and small) eddy turbulence. This last feature results from the fact that RAPTAD is a Lagrangian dispersion model. The weaknesses in the code include the following: (1) it has no provision for denser-than-air dispersion; (2) it lacks sufficiently refined spatial resolution to model short-duration episodic releases of vapor; (3) it requires extensive tailoring of the input data to reflect the climatic characteristics of a specific site; (4) it uses the hydrostatic approximation to flow and, therefore, may give incorrect results when the terrain is steep, winds are strong, and stability is high; and (5) the altemating direction implicit (ADI) numerical method used to solve the prognostic equations is diffusive.

Because the users guide was not complete at the time of the code evaluation, the problem evaluations focused more heavily on evaluating the code's functionality and capabilities, and the reasonableness of the final results rather than on attempting an extensive numerical comparison with the other dispersion codes in the study. An extensive number of experimental validations have been performed for HOTMAC, including air flow and $\mathrm{SO}_{2}$ concentration simulations (1992), coastal complex terrain simulations (1992), noctumal drainage flows, the 1982 ASCOT Bush Creek data simulations (1987), and the CAPTEX long-range transport data (1988). Air quality studies conducted with HOTMAC/RAPTAD have been verified against available data for Mexico City, Mexico; El Paso, Texas; and Santiago, Chile.

\section{INPUFF (Integrated Puff) - Version 2.3}

\section{Conditionally recommended for special SBD applications}

INPUFF is conditionally recommended for special SBD applications within the model's capability domain primarily because it is intended only for passive instantaneous release puff dispersion applications. The model is not capable of treating dense-gas dispersion.

NPUFF was originally developed by EPA in mid-1980 to assist permit applicants in modeling the air quality downwind of incineration ships. The code has been revised several times, and the last revision (version 2.3) was released in September 1988. The code is considered easy to use. The model's technical report and users guide provide detailed discussions of the dispersion algorithm, modeling assumptions/limitations, and input requirements.

The major strength of the INPUFF code is its capability to simulate dispersion from multiple, semi-instantaneous/continuous, neutrally buoyant point sources (moving or stationary) over a spatially and temporally variable wind field. Other significant strengths are the model's capability to handle time-dependent release rates and estimate ground-level or aboveground 
concentrations at up to 100 receptor locations for the user-specified averaging time. The major weakness is that the code cannot model dense-gas dispersion because of the use of Gaussian puff models. Compared with other Tier I codes, test run results from INPUFF are considered reasonable considering that the code assumes all scenarios as neutrally buoyant point source releases. Model performance has not been compared against field data.

SCIPUFF (Second-Order Closure Integrated Puff) - Version 0.338

\section{Conditionally recommended for further review and evaluation}

SCIPUFF is conditionally recommended for further review and evaluation primarily because the version available for review had neither a users guide nor complete or final technical documentation. The model's fearures and attributes could eventually have special, but limited, utility in SBD applications (e.g., for uncertainty analysis). Review for broader SBD applications would be considered in future reviews if the model's planned upgrade includes ability to treat dense-gas dispersion.

SCIPUFF was onginally developed in the mid-1980s under the sponsorship of the Electric Power Research Institute and subsequently under the Defense Nuclear Agency. The model's draft technical report (April 1995) provides a detailed discussion of the model physics but does not sufficiently highlight the practical applications and limitations of the code. The present abbreviated form of the users guide lacks the detail required to aid the user in selecting input parameters or model options appropriate to specific applications.

The model's graphical user interface is designed to provide intended users (i.e., Department of Defense personnel) with a "user friendly" tool. The Hazard Assessment System for Consequence Analysis (HASCAL) front end to SCIPUFF provides an "operator-friendly" interface for entering model input parameters and displaying model results graphically. It also supports graphical displays of instantaneous concentration, integrated dose, and ground deposition in the form of contour plots superimposed on a vector-based, high-resolution digital base map. The major user-oriented shortcomings of the reviewed version of HASCAL/SCIPLFF are as follows: (1) output results can only be displayed as "on-screen" graphical contour plots for instantaneous concentration, integrated dose, and ground deposition (no tabulated model output summaries), (2) the HASCAL "front end" is more appropriate to nuclear and/or biological, rather than chemical material releases, and (3) the SCIPUFF-required available input parameter set for user entries is kept to a minimum (stored in or retrieved from pre-defined accident release scenarios).

The model system (HASCAL/SCIPUFF) is evolving rapidly, with release of system version 2.0 in October 1996 (SCIPUFF version 0.6). This version may have addressed the noted weaknesses and limitations inherent in the version reviewed by the CDCA Working Group. Because SCIPUFF is conditionally recommended for further review and evaluation, the model application results (with accident scenarios) are not presented in this report. Although SCPPUFF has not yet been subjected to an independent model performance evaluation, plans were initiated in November 1996 to conduct a study comparing field observations with model predictions. 


\section{SLAB (Dispersion Model for Denser-than-Air Releases) - Version June 1990}

\section{Conditionally recommended for SBD applications}

SLAB is conditionally recommended for SBD applications within its capability domains primarily because of the model's wide acceptance and application in regulatory and other situations. Also, in independent model performance evaluations SLAB has demonstrated its ability to predict concentration estimates for both dense- and passive-plume behavior that are within a factor of 2 of field observations. The model is also listed as an EPA "Appendix B" refined air quality model that may be considered for individual regulatory applications on a caseby-case basis (Guideline for Air Quality Modeling, EPA 1996b).

SLAB grew out of some basic research involving experiments with chemical releases at China Lake and the Nevada Test Site in the early 1980s. The code first became publicly available in 1985, primarily for applications involving evaporative emissions. The code can be obtained from the EPA SCRAM Bulletin Board (Web page access through BBS/TTN 2000 at: http:/www.epa.gov/SCRAM001).

SLAB is a well-documented dense-gas-dispersion model that is in wide use. Its major strength is its ease of use as a dense-gas model, with application to jets released at any angle, to ground-level evaporative area sources, and to instantaneous sources. It also contains a unique averaging-time algorithm that allows application of the model to time-varying source emission scenarios. A major weakness, for users involved with complicated release scenarios, is that SLAB does not calculate its own source emission rates. The user is required to specify an emission rate input. In the test cases for this evaluation, SLAB produced concentration predictions that were within a factor of 5 (on the average) of the predictions of ALOHA, DEGADIS, and HGSYSTEM (three other dense-gas models conditionally recommended for SBD) (see discussion in Section 5.2.2). Its dense $-g a s$ algorithms have been satisfactorily compared with field observations, and its passive or neutrally buoyant gas algorithms agree with standard dispersion formulas in common use by EPA and DOE.

\section{TSCREEN (Toxic SCREEN) - Version 94133}

\section{Conditionally recommended for special SBD applications}

TSCREEN is conditionally recommended for special SBD applications within its capability domain primarily because of its design as a screening tool, with capability in simulating impacts due to dense-gas dispersion.

TSCREEN was designed to be used as a screening model in evaluating the effects of a series of typical release scenarios. It was developed by Pacific Environmental Services, Inc. under contract to EPA. The model should be used with the Workbook of Screening Techniques for Assessing Impacts of Toxic Air Pollutants (revised) (EPA 1992). The workbook also serves as model documentation. TSCREEN is very easy to use. Problems can be set up quickly using the top-down menu prompts for input data. The screen displays are self-explanatory and on-line help is available. If a set of input data is entered that cannot be used by the model, an alternative configuration will be suggested that is similar. 
TSCREEN's major strengths are its user friendliness, its fast calculation time, and its variety of source configurations. Chemical data can also be entered and stored for future use. Input data sets can be retrieved, modified, and rerun. One weakness is its inability to run source configurations that are not described in the workbook. Only two of the seven test cases for this study could not be run. Since it is intended as a screening tool, TSCREEN does not have several functions typical of a full-featured model, such as capabilities to use specific meteorological data or to consider time-dependent releases. In most of the test cases for which it was used, TSCREEN calculated the highest results. It should be noted that TSCREEN is designed to select meteorological conditions that produce the worst-case results; these conditions are often different from those that were defined for the test cases and used by the other models. No history of comparison with field data was identified, but comparing it would be difficult because the user has no control over the meteorological conditions used in the calculations.

\section{VLSTRACK (Vapor, Liquid, and Solid Tracking) - Version 1.6}

\section{Not recommended for DOE SBD applications}

VLSTRACK is not recommended for DOE safety-basis documentation primarily because it is designed for special military applications. The VLSTRACK model (version 1.0) originally was developed in response to Navy requirements during Operation Desert Shield. Version 1.2 was a further development of version 1.0. Version 1.3 was developed in response to comments hy users. Then, major revision and enhancement of version 1.3 were undertaken in response to requirements of the Space and Naval Warfare Systems Command, U.S. Marine Corps, U.S. Army Space and Strategic Defense Command, U.S. Army Nuclear and Chemical Agency, and Defense Nuclear Agency. VLSTRACK 1.5 included all of the features of previous versions and added the capability of addressing many situations resulting from high-altitude intercepts of chemical or biological warheads. Version 1.6 has been developed in response to an independent technical review performed by the National Oceanic and Atmospheric Administration (NOAA). The NOAA reviewers found significant theoretical errors in the methodology developed for VLSTRACK. VLSTRACK 1.6 corrects these theoretical errors, integrates the features of all of the specialized versions, and adds some additional capabilities developed for these versions. Newer versions were issued during this study.

The documentation for the model is complete and discusses each input screen. The user interface consists of data entry windows that respond to either mouse or keyboard commands. There are separate windows for each type of input, and online help is available. Strengths of the model are in applications for munitions. In its present form, VLSTRACK is very difficult to apply to common chemical spills of the type generally evaluated hy DOE. Only two of the test cases for this study could be attempted with VLSTRACK. The VLSTRACK results for those test cases do not show the same downwind distribution as the other models. Generally the model predicts lower concentrations close to the source and higher concentrations farther out. No history of comparison with field data was identified. 


\subsubsection{Tier II Model Recommendations}

The Tier II reviews (evaluation but no execution with accident scenarios) were limited in scope and not intended to provide sufficient information to support a conditional recommendation for use from the working group. However, the Tier II reviews were sufficient to either recommend models for further review and consideration (Table 6.5) or not recommend them for use in SBD preparation.

\section{TABLE 6.5 Tier II Models Conditionally Recommended for Tier I Review Consideration}

\begin{tabular}{|c|c|c|}
\hline Model & $\begin{array}{l}\text { Noteworthy Factors } \\
\text { to Consider }\end{array}$ & $\begin{array}{l}\text { Limiting Factors } \\
\text { to Consider }\end{array}$ \\
\hline
\end{tabular}

AFTOX 4.0

ARAC II

$A Q P A C^{a}$

CTDMPLUS

HARM-II

GAUS1

RTVSM

VDI
129 chemical database/95th percentile toxic corridor

Particularly well suited to major releases with meso- and synoptic-scale transport

Incorporates a spills front end and treats dense vapor cloud dispersion effects

Explicitly accounts for flow and plume distortion in complex terrain without the need for a data and computer intensive 3-D wind field model (i.e., FEM3C)

Incorporates a spills front end and treats dense vapor clouds, aiso able to handle timedependent releases and chemical transformations

Easy to use screening tool that provides a wide variety of useful and quick calculations (e.g., building wake, stack downwash, wevdry deposition)

Capable of ureating dense vapor clouds, continued development support expected

Capable of treating the effecting of complex building structures on the transport and dispersion of dense vapor clouds.
No dense gas algorithm

Code not available for use on computers outside the ARAC center, and current version (II) does not have dense vapor cloud capability or a spills front end

Distribution and licensing for sales have been turned over to private sector

Developed for neutrally or positively buoyant routine steady-state releases

Small chemical library

Progranmed only for use on a HP-48 calculator and does not have dense vapor cloud capability

Site-specific empirical data from Dugway Proving Grounds may limit the extent of model application

Distribution and licensing for sales have been turned over to the private sector. Does not currently have a spills front end.

a AQPAC is now solely licensed for distribution as a proprienry model. Reviews of such models will be an issue to be addressed in any future Tier I model evaluations that are conducted under the APAC progran. 
The eight models recommended for review as Tier I codes in any future evaluations possess several noteworthy characteristics, or factors, that could provide the safety analyst with useful additional or alternative capabilities in conducting chemical accident consequence assessments. For example, four of the eight models have dense-gas capabilities. These noteworthy factors and any limiting factors that should also be considered are listed in Table 6.5.

The ARAC II model is a special case because it met all of the conditions for a Tier I review under this study, but it could not be reviewed as a Tier I code because of its lack of portability (cannot be used outside the LLNL ARAC Center). In part because of ARAC II's expected strong continued funding and development support and its national and international recognition in the atmospheric modeling community, the CDCA Working Group recommends that the APAC Executive Committee reopen the issue of code portability for further discussion. This point may be moot with regard to ARAC II (the code's CG-MATHEW/ADPIC module, version 4.0, is now available for purchase through the Energy, Science, and Technology Software Center at Oak Ridge National Laboratory), but portability will still be an issue for ARAC III when it is ready for applications at the ARAC Center.

The working group felt that the remaining Tier II models, MARSS, PUFF-PLUME, and EMGRESP would not warrant further review primarily because they either have too specialized or site-specific applications or are not expected to be maintained and kept up to date. Further discussion on the need for additional Tier I reviews is given in Section 6.4.

\subsection{CHEMICAL CONSEQUENCE ASSESSMENT MODELING NEEDS}

At the onset of the APAC methodology evaluation initiative, the APAC Executive Committee requested that each of the working groups identify areas where further research, development, and/or guidance would ensure continued improvement in the safe operation of DOE's current and future chemical and radiological installations. Upon completion of our work, the CDCA Working Group identified six research or training areas with potential for providing cost-effective means for achieving that goal. Our recommendations cover the need for further model review, evaluation, and development. We also address the need to train the safety analyst in consistent application of tools and approaches recommended in the guidance provided in this report. Finally, we identify a need to keep the guidance current and reflective of improvements in model and modeling approaches as the state-of-the-science evolves.

\subsubsection{Expand Tier I Model Reviews}

The Tier I and $\Pi$ reviews helped identify the capabilities, the limitations, and the strengths and weaknesses of models selected for this evaluation. Four codes were conditionally recommended for broad SBD applications. However, none of these codes is expected to be able to handle all of the potential accident scenarios that the safety analysis may need to address. Although limited in scope, the survey of safety analysts revealed that some applications may not be adequately addressed or addressed at all by the codes they were currently using, which included the models conditionally recommended in this report. For example, chemical accidents involving very hot plumes generated from flash or pool fires and boiling liquid, expanding vapor explosions (BLEVEs) involving generation and atmospheric injection of aerosols and/or vapors 
from debris fragments are not specifically or adequately addressed in the current set of conditionally recommended Tier I codes. In view of these limitations, it would be prudent to conduct additional Tier I model reviews. The 11 Tier I and II models recommended for further study in Section 6.3 should be considered in such reviews.

Inclusion of proprietary models in future reviews should be considered, including some of those models in the initial CDCA Working Group list of 134 codes given in Appendix D to this report. Some examples of additional public domain and proprietary models, not all of which are listed in Appendix D, that have special strengths, capabilities or features that should be included among models to be considered for further Tier I reviews include (1) UDM (formerly PHAST), (2) QUEST, (3) SLAM, (4) DRIFT, (5) ADREA-HF, (6) AERCLOUD, (7) CLOUD, (8) FIREPLUME, (9) HACS-RITRACE, (10) GASTAR, (11) HEAVY PUFF, (12) GReAT, and (13) SUPER-CHEMS. The working group strongly recommends that firm, precise, and clear criteria be used in selecting models for further Tier I reviews. The selection process should involve an initial screening of codes for capabilities that would enhance or supplement future chemical consequence assessments. An expanded survey of safety analysts would help focus on their special needs. The selection criterias should also require that to be eligible for Tier I consideration, a code should bave complete technical documentation and a users guide. An internal quality assurance certification and some level of performance evaluation should also be considered in the screeming process. Finally, refinements to the approach used for model reviews in this study, including consideration of the methods and findings of the SMEDIS program, need to be factored into any subsequent Tier I model reviews.

\subsubsection{Conduct a Systematic Model Performance Evaluation with Field and Laboratory Data}

The only way to determine how well a model really performs is to evaluate its predictions with field and laboratory data. As mentioned during our discussions of the models' predictions based on the hypothetical accident scenario test cases described in this report, it is possible to run 10 models on a test case and find that one model's predictions do not agree with the predictions of the other nine models. Although the results provide information on a comparative basis, they shed little light on model performance in absolute terms. It may be that the one outlier model is corect and the other nine models are not. Insight into this anomalous behavior would likely be revealed in an evaluation with real data.

The consensus of the CDCA Working Group is that the conditionally recommended Tier I models, including those recommended for further review, and some selected Tier II models reconmend for Tier I review should be evaluated using the Modelers' Data Archive (MDA). This archive is a data set used by Hanna et al. (1993) in their evaluations of dense-gas models. The MDA includes dense-gas field trials from Burro (LNG), Coyote (LNG), Desert Tortoise $\left(\mathrm{NH}_{3}\right)$, Goldfish (HF), Maplin Sands (LNG), and Thorney Island (Freon) experiments. The Prairie Grass passive-gas data set is also included. We recommend that standard statistical performance measures, such as those incorporated in the BOOT software and recognized by EPA and the American Meteorological Society, be applied in the model performance evaluations. 
The MDA data set should be augmented with the data from the new Kit Fox $\mathrm{CO}_{2}$ experiments when they become available in late 1997. Those field data are unique because they include studies of finite-duration releases over rough surfaces during stable, light-wind conditions. Other data that should be considered in the model performance evaluation are the European dense-gas field databases (e.g., the FL.ADIS ammonia experiments) and extensive laboratory databases (dense-gas experiments in wind tunnels at the University of Hamburg, The Netherlands Organization of Applied Scientific Research (TNO); U.S. Environmental Protection Agency; Ford Motor Co.; Atomic Energy Associates Consulting Services (AEA); Cermak, Peterka, and Petersen, Inc. (CPP); Colorado State University; and others).

In addition, the data set can be significantly expanded with extensive existing data contained in the European Union funded REDIPHEM project database. Data in research areas specific to improving the understanding of the effects from aerosols, obstacles, and complex terrain may be available in the future from ongoing data archiving efforts of recent and planned experiments. The curtent European SMEDIS project, for example, has special interest in data pertinent to these areas. Since SMEDIS and APAC have common goais and interests in the area of model performance evaluations and scientific model reviews (which in SMEDIS are included together under the umbrella of "scientific model evaluation"), opportunities may exist for future collaborations or information exchange.

Part of the new research study should be directed toward deciding on improved standards or criteria for assessing model performance. This should include the review of traditionally applied performance measures, such as those that have been endorsed by the EPA, and new measures that may be adopted in the SMEDIS "model validation" effort. In particular, SMEDIS does not intend to rank the models according to performance evaluation results. However, it may set targets for performance measures, i.e., a model would meet such targets if its performance measures for certain experiments fell within certain ranges of values (which could be reviewed from time to time). This procedure emphasizes absolute rather than relative performance of models. It is also suggesting that performance should be deemed acceptable if the targets are met. In practice it cannot be detemined beyond a certain point whether it is the model itself or the intrinsic uncertainty in such problems that is responsible for differences between predictions and data, and so further attempts to improve performance are not necessarily worthwhile.

\subsubsection{Address Model Development Needs}

On the basis of the Tier I and II model reviews and the survey of safety analysts, the working group identified several areas not adequately addressed or treated by any of the identified existing public domain models. In addition, no one model was capable of handling all of the potential accident scenarios that could be encountered. Some or all of the identified weaknesses of the models could be addressed through a targeted upgrade program (e.g., adding front ends to codes such as SLAB and DEGADIS) or through further model development, including incorporation of a comprehensive set of state-of-the-science features not present in any one code. Key modeling areas needing improvement include, but are not limited to, capabilities to account for the influence of (1) increased surface roughness typically associated with many DOE installation sites and industrial plants, (2) the aerodynamic effects of buildings and surounding terrain on near-surface releases, (3) short-duration releases, and (4) aerosol 
formation, evaporation, and removal, including wet and dry deposition. Each of these areas is briefly discussed below.

1. Air Dispersion Around Industrial Facilities - Occasionally cerain releases may be affected by building orientation that can channel flow into a building cavity recirculation region, producing elevated concentrations (see Item 2 below). However, in many instances, surface terrain features and obstacles to flow (e.g., tall grass, trees, plant equipment, buildings) enhance turbulence in a vapor cloud and thereby increase dispersion. These turbulence features are parameterized in a number of dispersion codes as a surface roughness length. This roughness length represents the average large-scale eddy size and is usually determined from measured wind profiles. Experimental data on surface roughness length exist, and attempts have been made by the American Petroleum Institute, the Petroleum Environmental Research Form, and the EPA to develop a quantitative methodology for estimating vapor dispersion in the presence of variable terrain features. A need currently exists to develop a parallel program for DOE/industrial complexes in order to estimate surface roughness length applicable to vapor sources at DOE facilities. Such a program may involve (1) collecting meteorological data and facility equipment dimensions for process facilities, (2) comparing surface roughness lengtlı estimates from on-site wind profiles to estimates from existing quantitative methods, and (3) developing a procedure for estimating surface roughness length from on-site data.

2. Building Wake Effects - Relatively few computer models are available for modeling the transport and diffusion of airborne vapor in the presence of obstacles to flow. The aerodynamic effects of buildings and surrounding terrain become especially important when the vapor source is located near such features since the vapor concentration profiles become very complex and dynamic. Many of the currently available public domain codes that treat small-scale turbulence in the presence of obstacles to flow are based on the $k-\varepsilon$ turbulence model. Simple analytical formulas (Wilson 1979 and Britter 1989a) that fill the data quite well are employed in the ISC and HGSYSTEM/UF $_{6}$ models. The $k-\varepsilon$ turbulence model offers a more comprehensive turbulent field predictive capability than $\mathrm{K}$-theory in the case of complex terrain or obstacles to flow. The level of accuracy, however, depends on the availability of experimentally determined parameters used in the model and on the appropriate selection and use of wall functions. $2 \downarrow$ Other difficulties in accounting for turbulent flow around

21 The standard $k-\varepsilon$ model is not valid in the immediate neighborhood of a wall, the ground surface, or any other barrier to flow in the viscous buffer layer. The computational domain of the model is terminated in the fully turbulent region at some prescribed distance from the wall, whereupon use is made of logarithrnic wall laws to bridge the gap between the no-slip condition at the wall and the fully turbulent fow conditions. 
objects include very extensive input requirements and the need for very extensive computing facilities (generally a supercomputer is required to get reasonably fast execution times). Development needs in this area include (1) removal of subjectivity in the selection of wall functions, (2) improvement to the turbulence closure models that relaxes the requirements for empirically determined parameters, (3) development of turbulence models that accept time-varying boundary conditions (e.g., a time-dependent vapor release rate), and (4) construction of user-friendly preprocessor packages that assist in input preparation and numerical grid generation.

3. Short Duration Releases - Many existing dispersion models are designed to assess either instantaneous releases or continuous long-term releases. Furthermore, many of the jet and plume release models are based in part on similarity theory, where the "tail effects" (i.e., the effects of discontinuities or sudden changes in concentration profile at the edges of the vapor cloud or plume) are neglected. A short-duration release may exhibit complex dynamic flow behaviors, especially in the early phases of the release, that are not well modeled as a mass-centered symmetrical puff or a steady-state continuous plume. Modeling a short-duration release as either an instantaneous release or a steady-state plume is in many instances overly conservative. Development of a short-term release model would need to consider solution techniques for the diffusion equations involving a timedependent, spatially distributed source.

4. Aerosol Formation, Evaporation, and Removal Mechanisms - Despite the large amount of research interest in the areas of theoretical and experimental liquid aerosols, no comprehensive aerosol physics model currently exists for use in vapor source-term and dispersion calculations. The lack of an accurate and reliable aerosol fornation model can lead to errors in simulating pressurized liquid releases and subsequent vapor dispersion. Work has been recently published on aerosol rainout and droplet size correlation and aerosol condensation and evaporation. This work has been compared to experimental programs sponsored by the AIChE (1998). A number of outstanding areas still require research and development, including (1) investigation of aerosol formation, focusing on effects of source geometry and release physics and thermodynamics, and the subsequent droplet size distribution, (2) development of efficient direct simulation algorithms that minimize the need for tunable parameters for use in the decoupling of complex interactive aerosol phenomena, and (3) improvement and validation of mass and heat transfer algorithms in an aerosol cloud. 


\subsubsection{Establish a Safety Analysis Clearinghouse Web Page}

An APAC homepage could be established on the World Wide Web to provide online resources to aid the safety analyst in selecting and applying chemical dispersion codes in support of SARs, BIOs, and JCOs. Figure 6.2 shows a possible prototype of such a Web page.

Many potential search and ranking options exist for the code database query summarized in one of the elements shown on the prototype homepage. The analyst might, for instance, wish to obtain a list of all codes with a specific modeling capability (e.g., "list all chemical dispersion codes that accept a time-dependent, dense-gas source term"). The datahase search engine could also be designed so that the list of candidate codes to support a particular application would be based upon a graded search involving a list of desired modeling capabilities and application at the Savannah River Site, for example. The final list of identified codes could then be ranked on the basis of the number of "hits" registered on the modeling capabilities list and the relative importance of each matched item.

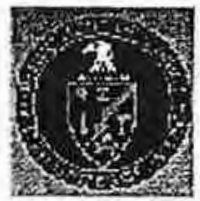

\section{APAC \\ Accident Phenomenology and Consequence Methodology Evaluation}

About APAC - Background, objectives, and general information on the APAC program

Working Groups - List of working groups, with links to group pages containing code evaluation summaries

Codes - Complete hist of codes evaluated under APAC program, with links to code summary pages, or go directly to a specific code page
Code Database Search - Query the APAC code database for specific modeling criteria. This search could be used to summarize modeling features for a single code and to perform code-to-code feature comparisons

General Search - Search the APAC webspace by keyword

Download documents - Download working group code evaluation reports and other general APAC documents

FIGURE 6.2 Possible Prototype APAC Web Page 
Document downloading from the homepage preferably would be limited to printable files only, in order to maintain control of document content and prevent the proliferation of unapproved document modifications. This control could be accomplished, for example, by using a portable document file (PDF) in conjunction with a document viewer such as Acrobar ${ }^{\Theta}$, which would also permit document searches and offer text recognition. Furthermore, various existing security measures, such as placing passwords on select pages or search features, that could offer some degree of protection by limiting the viewing audience with access to sensitive information.

Other possible features of an Internet-based CDCA support service might include:

- A clearinghouse to respond to safety analysts' questions about selection and application of chemical accident dispersion models.

- The capability to submit jobs to a designated computing facility for executing codes with special hardware requirements or that are maintained under configuration control. This service would, for example, accept the user-supplied input stream, run a script to execute the dispersion analysis code, and then transmit output files via E-mail back to the analyst. Furthermore, this service could be formalized in a manner to provide online code support if an analyst had difficulties in using the code or interpreting the results.

A safety analysis home page could be established to serve as a clearinghouse to respond to questions raised by safety analysts on the selection and application of chemical accident dispersion models specifically in support of SARs, BIOs, and JCOs. This report and guidance on specific technical and policy issues could be made available periodically.

\subsubsection{Establish Chemical Dispersion Modeling Workshop}

The CDCA Working Group has compiled a large amount of broad-based information covering a wide-range of technical topics relevant to dispersion analysis. Without some type of training program or workshop to effectively disseminate this information, it is probable that many safety analysts who read this report will not obtain the full benefit of its contents. Various types of training forums can be used to effectively communicate the important principles of chemical dispersion consequence assessment. For this type of material and the targeted audience, a workshop forum would be most appropriate. Accordingly, the CDCA Working Group recommends that a comprehensive workshop be designed, developed, and delivered to provide safety analysts with the skills needed to perform consistent and credible safety analyses of accidents involving chemical releases. The workshop should be designed to assist the analyst with code selection and to address the multiple release scenarios of hazardous chemical compounds, the various transport and dispersion characteristics of atmospheric redistribution, and the subsequent health effects to on-site and off-site receptors.

DOE's Office of Nonproliferation and National Security has already developed a threeday Consequence Assessment Workshop, which has been conducted at the Idaho National Engineering and Environmental Laboratory and the Rocky Flats Environmental Technology Site. 
The Subcommittee on Consequence Assessment and Protective Actions (SCAPA) is now considering expanding this workshop to five days and broadening the scope to include the following topical areas:

- Chemical release source term (gas/liquid tank releases, liquid pool releases, pipe releases, cryogenic and denser-than-air releases, and multicomponent releases);

- Fire release source term;

- Energetic event source term (detonations, deflagrations, BLEVEs);

- Chemical dose overview (types of exposure limits, types of toxicity data, dose time, entry route, body weight, and breathing rate adjustments);

- Temporary emergency exposure levels (TEELs); and,

- Chemical mixtures (types of health effects, past treatment, combined health effect index).

The CDCA Working Group recommends that DOE's Defense Program, Office of Engineering and Operations Support, explore possible collaboration with the Office of Nonproliferation and National Security in developing the atmospheric modeling/consequence assessment workshop.

\subsubsection{Consider Establishing a Joint Spills and CDCA Working Group}

Most chemical release accidents involve liquid spills. The test problems run for the Tier I models involved only vapor releases to the atmosphere. It was difficult to separate the liquid (evaporation) part from the chemical spill dispersion modeling calculations in many of the codes that incorporated both. While a few of the conditionally recommended models do not have spills front ends (i.e., SLAB and DEGADIS), the general consensus of the working group is that for liquid releases of any sizable magnitude, the use of a dense-gas model with a spills front end is highly recommended. If DOE chooses to implement some or all of the recommendations made by the CDCA and Spills Working Groups, in some cases it may be more efficient to complete certain tasks under a joint Spills and CDCA Working Group arrangement. That arrangement would enable the CDCA Group to run codes with recommended spills front ends starting with liquid releases rather than the vapor releases tested. We would not, however, necessarily preclude codes that do not have spills front ends from recommendation for Tier I review consideration or performance evaluation. 


\section{GLOSSARY}

Absolute humidity - The ratio of the mass of water vapor in moist air to the volume occupied by the mixture (i.e., the density of the water vapor)

Absorption -.. In meteorology, the retention of radiant energy by an irradiated substance and its conversion into other forms of energy.

Adiabatic (process) - Without exchange of heat across the boundaries of a system.

Adiabatic lapse rate $(\mathrm{dT} / \mathrm{dz})$ - The rate of decrease of temperature with height of a parcel of dry air lifted adiabatically. This rate is equal to $\mathrm{g} / \mathrm{C}_{\mathrm{p}},\left(9.8^{\circ} \mathrm{K} / \mathrm{km}\right)$ or a temperature decrease of about $1^{\circ} \mathrm{C}$ for each $100 \mathrm{~m}$ rise in elevation, where $\mathrm{g}$ is the acceleration of gravity and $\mathrm{C}_{\mathrm{p}}$ is the specific heat of air at constant pressure. A wide range of temperature gradients are observed in the atmosphere. The average lapse rate in the troposphere, which represents a balance between vertical mixing processes and radiative heat exchanges, is $-0.65^{\circ} \mathrm{C} / 100 \mathrm{~m}\left(-3.5^{\circ} \mathrm{F} / 1,000 \mathrm{ft}\right)$. The dry adiabatic lapse rate is $-1^{\circ} \mathrm{C} / 100 \mathrm{~m}$ $\left(-5.4^{\circ} \mathrm{F} / 1,000 \mathrm{ft}\right)$. The average lapse rate is closer to the dry adiabatic lapse rate.

Advection - The transport of a fluid property by bulk motion of the fluid, sometimes called convection in engineering terminology.

Aerosol - A solid or liquid particle existing as a dispersed phase in the atmospheric medium.

Albedo - The percentage ratio of radiant energy reflected by a body, especially a planetary surface, to that incident upon it (cf. reflectance). Albedos of uniform surfaces, such as fresh snow $(80-90 \%)$ or green forest $(5-10 \%)$ have been measured. The albedo of planet earth is estimated from satellite data to be about $30 \%$.

Averaging time - the time interval over which the instantaneous vapor concentration, or any other quantity of interest, is averaged.

Baroclinic - An atmospheric state that is not barotropic, in which temperature gradients exist in constant pressure surfaces. A consequence is the variation of the geostrophic wind with height.

Buoyancy-dominated plume - The buoyancy flux (proportional to the product of temperature difference between vapor and ambient air and the volume flux of a plume) of a continuous vapor release exceeds the momentum flux (product of the plume vertical speed and the volume flux). Examples of buoyancy-dominated plumes include the combustion products from medium-sized fires and releases of a chemical gas/vapor with a molecular weight less than that of air. 
Beyond design basis incident (BDBI) - Chemical accident release scenario with the largest potential impact that might credibly occur during facility operations. For SARs, JCOs, or BIOs, DBI should be conservative even if such incidents are quite rare.

Boussinesq approximation - The first or one of the first attempts to develop a mathematical description of turbulent stresses sought to mimic the molecular gradient-diffusion process. To do this, Boussinesq (1877) introduced the concept of eddy viscosity. The Boussinesq eddy-viscosity approximation solved the algebraic model of turbulence (simplest of all turbulence models) to compute the Reynolds stress tensor as the product of an eddy viscosity and the mean strain-rate tensor. Eddy viscosity is often computed in terms of a mixing length, for computational efficiency, which is analogous to the mean free path in an air parcel. In contrast to molecular viscosity, which is an intrinsic property of the fluid, eddy viscosity (and hence mixing length) is a property of the flow. Algebraic models require advanced specification of the eddy viscosity and mixing length parameters. Because of the dependence of these parameters on the particular flow under consideration, these models are by definition incomplete models of turbulence.

Co-located worker - A facility worker who is in the vicinity of a hazardous release (toxic, flammable, or explosion). For example, a co-located worker may be in the same building but another room than that in which a toxic chemical release was initiated. Another example is a worker who during an ex-facility chemical release is on-site, but may be several hundred meters away from the vapor source. In some parlance, the definitions of the co-located and local worker (see definition below) are reversed from those given here.

Computational Fluid Dynamics ( $C D F$ ) - A distinct approach to investigating the motion of a fluid under the action of applied forces, usually as a result of flow interaction with solid surfaces. High-speed computers are used to numerically solve a set of partial differential equations that approximate the physics of flow, whereas applied theoretical fluid dynamics in general uses simplifying assumptions to the governing equations of flow in order to obtain a closed-form analytic solutions to a given problem. The FEM3C model was the only CFD evaluated by the CDCA Working Group.

Conservative bias - A tendency to overestimate rather than underestimate.

Convective velocity scale (w*) _ One of several key parameters, which include mixing-layer depth $\left(z_{i}\right)$ and convective temperature scale $\left(T_{*}\right)$, used in characterizing (convective scaling) turbulent diffusion in the convective boundary layer (CBL). Applications of convective scaling theory to diffusion in the CBL first began with Deardorff's use in laboratory tank experiments in 1970 . The convective scaling velocity is defined as:

$$
\mathrm{W} *=\left(\frac{g}{T} \overline{w^{\prime} T^{\prime}} z_{i}\right)^{1 / 3}=u *\left(-\frac{z_{i}}{0.4 L}\right)^{1 / 3}
$$


where

$$
\begin{aligned}
& \frac{g}{T} \overline{w^{\prime} T^{\prime}}=\text { surface buoyancy flux } \\
& \text { g\& } \mathrm{T} \quad=\text { acceleration due to gravity and absolute temperature } \\
& \overline{w^{\prime} T^{*}} \quad=\text { kinematic sensible heat flux at surface } \\
& \overline{w^{\prime}} \quad=\text { vertical velocity variance } \\
& u * \quad=\text { friction velocity (defined below) }
\end{aligned}
$$

Coriolis force - A virtual force added to a force balance equation accounting for the rotational effects of the earth so that the force equations are derived in an inertial frame of reference (i.e., no rotation).

Default value - A value prescribed for a model parameter in the absence of data directly relevant to the assessment situation.

Dense gas/vapor - Gas or vapor emissions that exhibit a density significantly greater than that of the surrounding ambient air at existing temperature conditions (synonymous with heavy gas).

Deterministic model - A model whose output is predetermined by the mathematical form of its equations and the selection of a single value for each input parameter. The governing set of equations is solved to provide a point-value estimate of the dispersion characteristics of a contaminant in the statistical mean. The fluctuation in these quantities due to random effects (e.g., higher order turbulence, highly variable meteorological conditions) is either neglected or assumed to conform to some predictive functional relationship in the governing equations.

Dispersion coefficient $(\sigma)$ - A measure of the spreading of a flowing substance, from a continuous release, due to the nature of the porous medium in the lateral and vertical directions.

Entrainment - Generally refers to the mixing of surrounding air with a gas/vapor cloud, plume, or jet due to such physical phenomena as advection (i.e., spatial variation in temperature of a medium through which a parcel of vapor is moving), momentum exchange induced by shear flow, molecular transport across a flow boundary, or turbulent (e.g., path of an individual parcel is random) mixing.

Eulerian coordinate system - A system of computing particle diffusion where the coordinate system is defined independently of the motion of the tracer particles. Therefore, if the coordinate frame is defined such that the particle is stationary under "zero" wind conditions, then the position of the particle varies continuously in the coordinate system for all times when the wind speed is not zero. 
Extrapolation - The projection of model calculations to situations outside the realm of past experience or known data. Model calculations performed within the realm of experience and pertinent data are considered to be interpolations unless verified by measurement. The Pasquill-Gifford Turner $\sigma_{\mathrm{y}}$ and $\sigma_{\mathrm{z}}$ values are extrapolated at distances beyond the field studies on which they are based.

Flashing - Flashing is a process whereby a portion (or in some cases all) of a liquid suddenly vaporizes. A liquid that experiences a sudden change in pressure such that its temperature is above the boiling point at the new pressure conditions will undergo a phase change to gas.

$f-N$ Curves -- A shorthand notation for a curnulative complementary distribution function: a plot of the cumulative frequency (e.g., events/year with severity exceeding $N$ ) as a function of $N$ (number of injuries or fatalities).

Fumigation effects - Fumigation refers to the process whereby an elevated plume trapped in a stable (or neutral) flow is entrained into a developing convective boundary layer and undergoes rapid vertical mixing. This situation results in a concentrated plume at the ground level, where the fumigation occurs. Fumigation can also occur during seabreeze conditions when the elevated plume intersects the thernal internal boundary layer (TTBL).

Friction velocity - A measure of the mechanical turbulence and a direct measure of the frictional forces of the wind in the surface boundary layer (SBL). It can be thought of as representing the consequence of Reynolds stresses, which cause velocity fluctuations to transport momenrum across the surface.

Assuming the viscous stress in the SBL is constant in magnitude and direction, it is common to define a shear stress reference velocity (known as the friction velocity or mechanical-turbulence velocity scale) that is constant with height (assuming $\tau$ and $\rho$ are constant with height) in the SBL. A mathematical form typically used to define friction velocity is the square root of the ratio of tangential shearing stress to air density:

$$
u *^{2}=\tau / \rho
$$

where

$$
\begin{aligned}
& \tau=\mu \mathrm{d} u / d z=\rho v \mathrm{du} d \mathrm{dz}=1 / 2 \rho \mathrm{C}_{\mathrm{D}} \vec{u}^{2} \\
& \rho=\text { atmospheric density } \\
& \mu=\text { eddy viscosity } \\
& v=\text { kinematic viscosity } \\
& C_{D}=\text { drag coefficient } \\
& \bar{u}=\text { mean reference wind speed, usually taken at } 10 \mathrm{~m}
\end{aligned}
$$


Friction velocity is a relevant and key scaling parameter for modeling diffusion in the surface layer. Good estimates of $\mathrm{u} *$ depend on knowledge of the nature of the surface (e.g., heat fluxes), the magnitude of the mean velocity $(\bar{u}(\mathrm{z})$ ), and temperature differential with height. Without this knowledge, surface stress $(\tau)$ can be approximated with the expression above and the following general drag coefficient estimates (Randerson 1984):

$$
\begin{aligned}
& C_{D}=10^{-3}-\text { smooth ocean } \\
& C_{D}=10^{-2}-\text { bushy canopy } \\
& C_{D}=10^{-1}-\text { urban environment }
\end{aligned}
$$

Gaussian model - A pollutant diffusion model based on an assumption of stationary, homogeneous turbulent flow. The distribution of material in the plume or puff is assumed to be Gaussian in shape in each spatial dimension.

Gaussian plume -- A diffusion model for continuous vapor releases in which the lateral distribution of vapor concentration (i.e., variation of the concentration as a function of radial distance measured perpendicular to the plume centerline) follows a normal or Gaussian distribution.

Gaussian puff - A diffusion model for "instantaneous" or short-duration vapor releases where the radial distribution of vapor concentration (i.e., variation of the concentration as a function of radial distance from the cloud centroid) follows a normal or Gaussian distribution.

Gradient transport - - An approach in dispersion analysis in which it is assumed that turbulence causes a net movement of vapor along the gradient of vapor concentration at a rate that is proportional to the magnitude of the gradient. The basic equation in Cartesian coordinates is given by

$$
\frac{\partial \chi}{\partial t}+u \frac{\partial \chi}{\partial x}+v \frac{\partial \chi}{\partial y}+w \frac{\partial \chi}{\partial z}=S+\frac{\partial}{\partial x} K_{x} \frac{\partial \chi}{\partial x}+\frac{\partial}{\partial y} K_{y} \frac{\partial \chi}{\partial y}+\frac{\partial}{\partial z} K_{z} \frac{\partial \chi}{\partial z}
$$

where $c$ denotes vapor concentration, $u, v, w$ represent the mean wind speeds in the $x$-, $y$-, and $z$-directions, and $S$ is the vapor source-term. Wind fluctuations and turbulence are implicitly captured in the functional form of the $K$ coefficients in the above equation.

Hazard zone - Alternatively referred to as a plume footprint, toxic corridor, or concentration isopleth - the region of a plane at a fixed elevation above the ground and parallel to the ground surface where the concentration exceeds a given minimum threshold.

Homogeneity - The properties, or conditions, of isotropy or anisotropy are constant from point to point in the atmosphere. 
Hydrostatic approximation (mesoscale models) -- The vertical acceleration and vertical components of the Coriolis and frictional forces are neglected in comparison to the acceleration of gravity. This assumption is not valid for small-scale or turbulent components of hlow.

Interdiction - Refers to the physical phenomenon whereby a vapor plume penetrates either partially or in full the inversion layer and is subsequently trapped in the upper stable layer (see also fumigation).

Inversion layer - A region of air in which the temperature increases with increased distance from the ground. More than one inversion layer may exist at an instant in time for a given site (i.e., the inversion layers are "stacked" — each separated by a single mixing layer (region of the atmosphere where the temperature decreases with an increase in altitude).

K-Theory - Same as gradient transport theory (see above).

$K-E$ Theory - turbulent flow theory that models the diffusivities with variables characterizing the turbulent structure, such as the turbulent kinetic energy, $K$, and its rate of dissipation, E. Compared with models based on the $\mathrm{K}$-theory, $\mathrm{K}-\varepsilon$ models solve two additional differential equations - one for turbulent kinetic energy and one for its rate of dissipation.

Lagrangian coordinate system - Flow statistics (e.g., velocity) for a given particle are measured locally by following the successive movements of the particle. Therefore, the coordinate frame of the "observer" who is conducting the measurements is moving at the same rate and direction as the subject particles for all time.

Land-sea interaction - The interaction of air masses and wind fields at the interface between coastal land masses and large bodies of water. The interface of these two different air mass/wind fields is called the thermal internal boundary layer (TIBL).

Lift-off (convective/surface) - A phenomena whereby under convective atmospheric conditions, surface-induced turbulence enhances the vertical spread of a vapor cloud or plume generated from a ground-level release. The net effect is a rise in the vapor-cloud or plume from the surface.

Lift-off (exothermic/chemical) - A phenomena whereby chemical reactivity within a vapor cloud results in an exothermic reaction that in turn increases the gas/vapor temperature and induces positive buoyancy in the plume. The net effect is to transform a dense-gas or neutrally buoyant plume or cloud into its buoyant, upward-rising counterpart.

Local worker - A facility worker who is very close to a hazardous release (e.g., toxic, flammable, or explosive). An example would be a facility worker who is in the same room as a yapor source at the time of release or within 10 to $50 \mathrm{~m}$ of an ex-facility chemical release. Note that in some parlance, the terminology of local and co-located worker (see above) is reversed from that given here. 
Mesoscale model - A transport and dispersion model that considers particle motion on a spatial grid resolved to between 10 and $500 \mathrm{~km}$ between each successive node. Mesoscale motions include phenomena such as sea breezes, mountain-valley wind systerns, squall lines, cloud clusters, tomadoes, and orographic features such as standing waves.

Mixing depth $\left(\mathbf{z}_{i}\right)$-- Sometimes also referred to as the mixing layer - the region of air bounded by the ground surface at the lower level and extending to the top of the planetary boundary level, namely the lowest 1 - to $2-\mathrm{km}$ layer of the atmosphere experiencing surface effects through vertical exchange of momentum, heat, and moisture. This region is typically turbulent during the daytime. The mixing depth often decreases during nightime conditions unless high winds are present. Many times it is assumed that the mixing depth is equal to the vertical distance to the bottom of the first inversion layer. Although this assumption is usually valid for daytime conditions, the mixing depth may be much greater than the height of the first inversion layer during calm, nighttime conditions, when the inversion layer essentially extends to ground level.

Momentum-dominated plume - The momentum flux (product of the plume vertical speed and the volume flux) exceeds the buoyancy flux (proportional to the product of temperature difference between vapor and ambient air and the volume flux of a plume) of a continuous vapor release. Examples of momentum-dominated plumes include vapor/gas emissions from a forced ventilation system and blowdown of a pressurized vessel.

Monin-Obukhov length (L) - A parameter related to the depth of the mechanically mixed layer near the ground surface. Its value depends essentially on only the heat flux between the ground and the air layer above and the friction velocity (defined as the ratio of the magnitude of the Reynold's stress vector to the density of the air, all evaluated at ground surface).

Neutrally buoyant gas/vapor - Gas or vapor releases that exhibit essentially the same material density as air and therefore behave as a passive tracer that follows the same bulk movements and behavior as the surrounding ambient air.

Nudging (also referred to as Newtonian relaxation) - Terms added to the conservation relations that "nudge" the solutions toward observed conditions. The values of the coefficients in these terms must be adjusted by the analyst and are used to remove inconsistencies in meteorological data to account for varying large-scale meteorological conditions, to overcome numerical errors, or to force the model to match field data.

Plume meander - The variation of a plume centerline trajectory due to time-dependent variations in the prevailing wind direction. The net effect is to increase the vapor concentration at the Iateral extremes of the cloud and decrease it at the centerline (centroid). Plume meander frequently occurs during nightime conditions with very light winds.

Positively buoyant gas/vapor - Gas or vapor releases that exhibit a density significantly less than that of the surrounding air at existing temperature conditions. 
Potential temperature $(\theta)$ - The temperature a parcel of air would reach if that parcel were brought up adiabatically from a pressure $p$ to a pressure of $10^{6} \mathrm{dynes} / \mathrm{cm}^{2}(1,000 \mathrm{mb})$.

$$
\theta \cong T+\left(g / C_{p}\right) z
$$

where

$$
\begin{aligned}
& \mathrm{z}=\text { the height above mean sea level in meters, } \\
& \mathrm{g}=\text { the acceleration due to gravity, } g=9.8 \mathrm{~m} / \mathrm{s} \\
& \mathrm{C}_{\mathrm{p}}=\text { the specific heat at constant pressure, } \mathrm{C}_{\mathrm{p}}=10^{7} \mathrm{ergs} / \mathrm{g}-\mathrm{K} \\
& \mathrm{T}=\text { the air parcel temperature in } \mathrm{K}
\end{aligned}
$$

The adiabatic potential temperature gradient, $\mathrm{d} \theta / \mathrm{dz}$, is zero.

Probit - Otherwise known as probability units - used to quantify the probability of injury or fatality due to an exposure to a hazardous event. A log-normal distribution expresses probability in units of standard deviation. The integration of the response curve or lognormal distribution produces a straightline plot of probability versus the log of the toxic load. The general form of the equation for evaluating the probability of impact (P) is given by:

$$
P=\frac{1}{\sqrt{2 \pi}} \int_{-\infty}^{Y-5} e^{-u^{2} / 2} d u
$$

where $Y$ denotes the actual value of the probit, generally expressed as

$$
Y=a_{1}+b_{2} \ln (V) \text { or } Y=\mathrm{a}+\mathrm{b} \ln \left(\mathrm{C}^{\mathrm{n}} \mathrm{t}\right)
$$

where $a_{1}, b_{2}$ and n are constants fitted to data typically by means of a maximum likelihood estimation, $\mathrm{V}$ is some hazard exposure value, e.g., vapor concentration or inhalation dose, $\mathrm{C}$ is the concentration and $\mathrm{t}$ is the exposure time.

Prognostic model - A model that uses the governing equations of atmospheric fluid flow along with initial meteorological data and imposed boundary conditions to generate a solution to predict what the meteorological conditions will be at some future time.

Public receptor - Generally refers to any individual not located within the facility site boundary at the time of a hazardous release (toxic, fire, explosion) that may incur the risk of exposure from a release occurring on-site. 
Random walk - Dispersing particles travel in straight lines between collisions with the dispersing medium, during which the velocities are independent of previous velocities and depend solely on the turbulence statistics at the point of collision.

Resuspension - Re-entrainment of solid particulates (either by dry deposition or wet deposition [i.e., adsorption by liquid film or pool]) into the wind field, leading to subsequent downwind dispersion.

Risk analysis - The development of a quantitative estimate of risk on the basis of science or engineering evaluations and mathematical techniques for combining estimates of incident consequences and frequencies.

Risk assessment - The process by which results of a risk analysis (i.e., risk estimates) are used to make decisions, either through the relative ranking of risk reduction strategies or through comparison with risk targets.

Roughness parameter or length $\left(\mathbf{z}_{\mathbf{0}}\right)$ - A'measure of the effect of ground surface roughness on vertical wind profile (integration constant in logarithmic wind profile)

$$
\bar{u}=\frac{u_{*}}{k} \ln \left(\frac{z}{z_{o}}\right) .
$$

Mean wind speed is zero at this reference height. It is also used to represent the size of turbulent eddies at the ground surface. This parameter is not only a function of the height of obstacles to flow, but also depends upon the shape and spacing of the surface features. The most reliable value for roughness length may be obtained from measured wind profiles by determining the average elevation where the wind speed approaches zero. Nonetheless, it has become customary to base roughness length on ground cover characteristics. Typical values of $\mathrm{z}_{0}$ and $\mathrm{u} *$ for a variety of surface types are provided in Section 6.1.

Second-order closure - One approach to modeling atmospheric turbulence. The dependent variables in the governing equations of transport and diffusion are decomposed into mean and fluctuating components. The resulting equations are then integrated to obtain statistical equations to describe the evolution of mean quantities. As a result of the averaging, information is lost, and so the turbulent velocity statistics, present in the form of cross products in a second-moment expansion, are related to the rate of dispersion in order to close the system (i.e., complete the system of equations, or a sufficient number of equations for all unknowns, so that the solutions are unique).

Segmented Gaussian plume/puff - A computational approach whereby a Gaussian plume or puff is spatially segmented into individual volume sources, with each segment generating a concentration field. 
Similarity plume/puff - Dispersion models that assume that specific variables of interest (e.g., concentration distribution) retain the same functional form independently in space and/or time when other select variables are held constant. In particular, such models are predicated on the assumption that the vapor concentration maintains a Gaussian distribution throughout all time (although the standard deviations themselves generally vary with downwind distance).

Site acquisition of meteorological (SAM) data - Meteorological data that are continuously being recorded on a specific site by a network of meteorological towers.

Spills - Any release of a hazardous material into the ambient environment, including liquid chemical spills and evaporation, pressurized liquid/gas releases, solid spills and resuspension/sublimation, and resuspension of particulate matter from liquid spills.

Stochastic model - Any model whose input and output are expressed as random variables (contrast with deterministic model). An approach taken in transport and diffusion where select parameters in the goveming equations (e.g., wind trajectory, turbulent length scales, etc.) take on the roles of random variables whose successive values in time are sampled from an appropriate underlying distribution. The numerical values obtained from a stochastic model include not only the mean or expected average value, but also a measure of variability in the result within prescribed confidence limits.

Straight-line Gaussian plume/puff - Gaussian plume and puff models in which the dispersion parameters (typically referred to as the $s$ factors) are only a function of the distance and terrain features occurring on a straight 'line-of-sight' between the vapor source point and the receptor location. Such models generally cannot assess the effects of plume meander (i.e., variable wind trajectory) on the subsequent downwind vapor concentration. NRC Gaussian models have the ability to incorporate a "meander fraction" by expanding the lateral turbulence.

Turbulence - No generally accepted definition exists. Usually described by its characteristics: (1) chaotic or random fluid velocity in space and time, (2) flow in three dimensions with gradients in all directions, (3) nonlinear phenomenon, (4) kinetic energy transmitted to successively smaller length scales, and (5) diffusive and intermittent flow.

Two-phase - A vapor-liquid mixture that occurs when a portion of the fluid consists of vapor bubbles suspended in liquid. Two-phase releases often occur when a liquid or vapor processed or stored at temperatures approaching the saturation temperature and at pressures exceeding the saturation pressure are released from containment and experience a sudden drop in pressure.

Uncertainty - The lack of sureness or confidence in the predictions of models.

Uncertainty analysis - Analysis of the uncertainty in model predictions. 


\section{REFERENCES}

AIChE/CCPS - See American Institute of Chemical Engineers, Center for Chemical Process Safety.

AJHA - See American Industrial Hygiene Association.

American Industrial Hygiene Association, 1996, The AIHA Emergency Response Planning Guidelines and Workplace Environmental Exposure Level Guides Handbook, Fairfax, Va.

American Institute of Chemical Engineers, Center for Chemical Process Safety, 1989, Guidelines for Chemical Process Quantitative Risk Analysis, New York, N.Y.

American Institute of Chemical Engineers, Center for Chemical Process Safety, 1995, Concentration Fluctuations and Averaging Time in Vapor Clouds, New York, N.Y.

American Institute of Chemical Engineers, Center for Chemical Process Safety, 1996, Guidelines for Use of Vapor Cloud Dispersion Models, 2nd Edition, Publication 1439, New York, N.Y.

American Institute of Chemical Engineers, Center for Chemical Process Safety, 1998, Design and Review of CCPS Aerosol Field Tests, Parts 1 and 2 (in final review and printing, publication expected in January 1998), New York, N.Y.

American National Standards Institute, 1984, American National Standard for Determining Meteorological Information at Nuclear Power Sites, ANS-2.5, Aug.

American Petroleum Institute, 1996, A Guidance Manual for Modeling Hypothetical Accidental Releases to the Atmosphere, Publication 4628, American Petroleum Institute, Health and Environmental Sciences Department, Washington, D.C.

ANSI — See American National Standards Institute.

API - See American Petroleum Institute.

Banerjee, S., et al., 1994, "CLOUD: A Vapour - Aerosol Dispersion Model Accounting for Plume 3D Motion and Heat and Mass Transfer Between Phases," Journal of Hazardous Materials 46:231-240.

Bettis, R.J., et al., 1987, “Two Phase Flashing Release Following Rapid Depressurization due to Vessel Failure," Hazards from Pressure, Industrial Chemical Engineering Symposium Series No. 102, American Institute of Chemical Engineers, pp. 247-263.

Boussinesq, J., 1877, "Theorie de l'Ecoulement Tourbillant," Mem, Presentes par Drivers Savants Acad. Sci. Inst. Fr. 23:46-50. 
Bowman, W.A., 1996, "Maximum Ground-Level Concentrations with Downwash: The Urban Stability Mode," Joumal of Air and Waste Management, 46:615-620.

Brereton, S., 1995, "Communication of Interface Issues among Working Groups," memorandum to distribution, Lawrence Livermore National Laboratory, June 30.

Brereton, S., et al., 1996, Spills Working Group Draft Report, U.S. Department of Energy, Sept.

Briggs, G.A., 1975, "Plume Rise Prediction," Lectures on Air Pollution and Environmental Impact Analyses, American Meteorological Society, Sept. 29 through Oct. 3, Boston, Mass., pp. 59-111.

Briggs, G.A., 1985, "Analytical Parameterizations of Diffusion: The Convective Boundary Layer," Joumal of Climate and Applied Meteorology 24:1167-1186.

Brighton, P.W.M., 1989, The Effects of Natural and Man-Made Obstacles on Heavy Gas Dispersion, Safety and Reliability Directorate, U.K. Atomic Energy Authority, Culceth, Warrington, U.K.

Brighton, P.W.M., et al., 1994, "Comparison of Heavy Gas Dispersion Models for Instantaneous Releases," Joumal of Hazardous Materials 36:193-208.

Britter, R.E. 1982, Special Topics on Dispersion of Dense Gases, Report on Contract No. 1200/01.01. Research and Laboratory Services Division, Health and Safety Executive, Sheffield, England.

Britter, R.E., 1989a, Experiments on Some Effects of Obstacles on Dense Gas Dispersion, SRD R-407, U.K. Atomic Energy Authority, Culceth, Warington, U.K.

Britter, R.E. 1989b, "Atmospheric Dispersion of Dense Gases," Ann. Rev. Fluid Mech. 21:317344.

Britter, R.E., and J. McQuaid, 1988, Workshop on the Dispersion of Dense Gases, HSE Report No. 17/1988, Health and Safety Executive, Sheffield, United Kingdom.

Britter, R., et al., 1995, Atmospheric Dispersion Modeling: Guidelines on the Justification of Choice and Use of Models and the Communication and Reporting of Results, a policy statement issued by the Council of the Royal Meteorological Society, Reading, U.K., May.

Cagnetti, P., et al., 1988, "An Atmospheric Diffusion Study on a local Scale at a Coastal Site," Atmospheric Environment 22:1051-1059.

Chrysikopoulos, C.V., et al., 1992, "A Three-Dimensional Steady-State Atmospheric Dispersion Model for Emissions from a Ground-Level Area Source," Atmospheric Environment 26A(5):747757. 
Craig, D.K., 1996, "ERPGs AND TEELs for Chemicals of Concern at SRS (Rev 11)," memorandum to distribution, Westinghouse Savannah River Corp., Oct.

Craig, D.K., et al., 1995, "Alternative Guideline Limits for Chemicals without Emergency Response Planming Guidelines," American Industrial Hygiene Association Joumal, Vol. 56, Sept.

Cramer, H.E., 1976, "Improved Techniques for Modeling the Dispersion of Tall Stack Plume," in Proceedings of the Seventh International Technical Meeting on Air Pollution Modeling and Its Application, No. 51, NATO/CCMS, pp. 731-780.

Design Institute for Emergency Relief Systems, American Institute of Chemical Engineers, 1992, Emergency Relief System Design Using DIERS Technology: The Design Institute for Emergency Relief Systems (DIERS) Project Manual, New York, N.Y.

DiCristofaro, D.C., and J.S. Touma, 1992, "Description and Sensitivity Analysis of the Shoreline Dispersion Model (SDM)," Proceedings of the 19th Intemational Technical Meeting of NATO-CCMS on Air Pollution Modelling and its Application, G. Kallos and H. van Dop (Eds.), Plenum Press, pp. 153-162.

DIERS/AIChE - See Design Institute for Emergency Relief Systems, American Institute of Chemical Engineers.

Draxler, R.R., 1976, "Determination of Atmospheric Diffusion Parameters," Atmospheric Environment 10:99-105.

EPA - See U.S. Environmental Protection Agency.

Evans, M., 1994, Quality Assurance of ALOHA, draft report, National Oceanic and Atmospheric Administration, Seattle, Wash., July.

Fannelop, T.K., and F. Zumsteg, 1984, "Special Problems in Heavy-Gas Dispersion," Heavy Gas and Risk Assessment, S. Hartwig (Ed.), D. Reidel Publishing Co., Dordrecht.

Fauske, H.K., and M. Epstein, 1988, "Source Term Considerations in Connection with Chemical Accidents and Vapor Cloud Modeling," Journal of Loss Prevention in the Chemical Process Industries 1:75-84.

Frost, W., et al., 1978, Engineering Handbook on the Atmospheric Environmental Guidelines for Use in Wind Turbine Generator Development, NASA TP-1359, NASA Lewis Research Center, Cleveland, Ohio.

Fthenakis, V.M. (editor), 1993, Prevention and Control of Accidental Releases of Hazardous Gases, Van Nostrand Reinhold, New York. 
Gifford, F.A., 1960, "Atmospheric Dispersion Calculations Using Generalized Gaussian Plume Model," Nuclear Safety 2(2).

Gifford, F.A., 1975, "Atmospheric Dispersion Models for Environmental Pollution Applications," pp. 35-58 in: Lectures on Air Pollution and Environmental Impact Analysis, American Meteorological Society, Boston, Mass.

Gifford, F.A., 1991, "Use of Routine Meteorological Observations for Estimating Atmospheric Dispersion," Nuclear Safety 2(4):47-51.

Golder, D., 1972, "Relations Among Stability Parameters in the Surface Layer," Boundary-Layer Meteorology 3:47-58.

Griffiths, R.F., and G.D. Kaiser, 1982, "Production of Dense Gas Mixrures from Ammonia Releases - A Review," Journal of Hazardous Materials 6:197-212.

Hanna, S.R., 1995, Personal communication; list of heavy gas models, July.

Hanna, S.R., and D.G. Strimaitis, 1990, "Rugged Terrain Effects on Diffusion, pp. 109-143 in: Atmospheric Processes over Complex Terrain, American Meteorological Society.

Hanna, S.R., et al., 1982, Handbook of Atmospheric Diffusion, DE82002045 (DOE/TIC-11223).

Hanna, S., et al., 1985, "Development and Evaluation of the Offshore Coastal Dispersion Model," Journal of Applied Meteorology 34:2259-2293.

Hanna, S.R., et al., 1991, "Evaluation of fourteen Hazardous Gas Models with Ammonia and Hydrogen Fluoride Field Data," Journal of Hazardous Materials 26:127-158.

Hanna, S.R., et al., 1993, "Hazardous Gas Model Evaluations with Field Observations," Atmospheric Environment 27A(15):2265-2285.

Havens, J., 1988, A Dispersion Model for Elevated Dense Gas Jet Chemical Releases, Vols. I and II, April, EPA-450/4-88-006.

Herman, M.N., 1987, Aerosol Formation and Subsequent Transformation and Dispersion, During Accidental Releases of Chemicals, American Petroleum Institute, July.

Hesse, D.J., 1991, "Modeling the Time and Spatial Dependence of the Transition from a Continuous Plume to a Detached Vapor Cloud," International Conference and Workshop on Modeling and Mitigating the Consequences of Accidental Releases of Hazardous Materials, May 20-24, New Orleans, American Institute of Chemical Engineers, New York, N.Y.

Hoot, T.G., et al., 1973, Wind Tunnel Tests of Negatively Buoyant Plumes, CER73-74TGHRNM-JAP-13, Colorado State University, Fort Collins, Colo. 
Houghton, D.D. (Ed.), 1985, Handbook of Applied Meteorology, Wiley-Interscience, John Wiley and Sons, New York.

Hovind, E.L., et al., 1979, Workshop on Amospheric Dispersion Models in Complex Terrain, EPA-600/9-79-041, U.S. Environmental Protection Agency.

Huber, A.H., 1977, "Incorporating Building/Terrain Wake Effects on Stack Effluents," Joint Conference of the Applications of Air Pollution Meteorology, Nov. 29-Dec. 2, pp. 353-356, Salt Lake City, Utah.

Huber, A.H., and W.H. Snyder, 1982, "Wind Tunnel Investigation of the Effects of a Rectangular-Shaped Building on Dispersion of Effluents from Short Adjacent Stacks," Atmospheric Environment 17:2837.

Hwang, R.R, and T.P. Chiang, 1986, "Buoyant Jets in a Crossflow of Stably Stratified Fluid," Atmospheric Environment 20(10):1887-1986.

Iannelo, V., et al., 1989, “Aerosol Research Program: Improved Source Term Definition for Modeling the Ambient Impact of Accidental Release of Hazardous Liquids," pp. 58-1 through 58-30 in: 6th International Symposium on Loss Prevention and Safety Promotion in the Process Industries, European Federation of Chemical Engineering, Oslo Norway, June 19-22, Norwegian Society of Chartered Engineers and Center for Chemical Process Safety (U.S.A.).

Kitamura, Y., et al., 1986, "Critical Superheat for Flashing of Superheat Liquid Jets," Industrial Engineering Fundamentals 25(2):206-211.

Kretzschmar, J.G., and I. Mertens, 1980, "Influence of the Turbulence Typing Schemes upon the Yearly Average Ground-Level Concentrations Calculated by Means of a Mean Wind Direction Model," Atmospheric Environment 14:947-951.

Leung, J.C., 1992, "Size Safety Relief Valves for Flashing Liquids," Chemical Engineering Progress, Feb., pp. 70-75 (Corrections: CEP, Mar. 1992, p. 108).

Leung, J.C., 1996, "Easily Size Relief Devices and Piping for Two-Phase Flow," Chemical Engineering Progress, Dec.

Leung, J.C., and M.A. Grolmes, 1988, "A Generalized Correlation for Flashing Choked Flow of Initially Sub-Cooled Liquid," AlChE Journal 34(4):688-691.

List, E.J., 1982, "Mechanics of Turbulent Buoyant Jets and Plumes," Turbulent Buoyant Jets and Plumes, W. Rodi (editor), Pergamon Press.

Luhar, A.K., and B.L. Sawford, 1985, "Langrangian Stochastic Modeling of the Coastal Fumigation Phenomenon," Joumal of Applied Meteorology 34:2259-2293. 
Lyons, W.A., 1975, "Turbulent Diffusion and Pollutant Transport in Shoreline Environments," Lectures on Air Pollution and Environmental Impact Analysis, American Meteorological Society, Boston, Mass.

Mazzola, C.A., and R. Addis, 1995, "Atmospheric Dispersion Modeling Resources," Second Edition, U.S. Department of Energy, Aiken, S.C., March.

Mills, M.T., and R.J. Paine, 1990, "A Survey of Modeling Techniques for Consequence Analysis of Accidental Chemical Releases to the Atmosphere," Reliability Engineering and System Safety 29:69-102.

Mitchell, A.E., 1982, "A Comparison of Short-Term Dispersion Estimates Resulting from Various Atmospheric Stability Classification Methods," Atmospheric Environment 16:765-773.

Mitchell, A.E., and K.O. Timbre, 1979, "Atmospheric Stability Class from Horizontal Wind Fluctuation," 72nd Annual Meeting Air Pollution Control Association, Cincinnati, Ohio, June 24.

Netterville, D.D.J., 1989, "Plume Rise, Entrainment and Dispersion in Turbulent Winds," Atmospheric Environment 24A(5):1061-1081.

Ooms, G., A.P. Mahiev, and F. Zelis, 1974, The Plume Path of Vent Gases Heavier than Air, First International Symposium on Loss Prevention and Safety Promotion in the Process Industries, C.H. Bushnan (editor), Ekevier Press.

Panofsky, H.A., and J.A. Dutton, 1984, Atmospheric Turbulence, Wiley, New York.

Pasquill, F., 1961, "The Estimation of the Dispersion of Windborne Material," Meteorology Magazine 90(1063):33-49.

Pasquill, F., 1974: Atmospheric Diffusion - The Dispersion of Windborne Material from Industrial and Other Source, 2nd Edition, John Wiley \& Sons, Ellis Horwood Limited.

Pielke, R.A., 1981, "Аn Overview of our Current Understanding of the Physical Interactions between the Sea- and Land-Breeze and the Coastal Waters," Ocean Management 6:87-100.

Ramsdell, J.V., Jr., 1990, "Diffusion in Building Wakes for Ground-Level Releases," Atmospheric Environment $24 \mathrm{~B}(3): 377-388$.

Randerson, D., et al., 1984, "Atmospheric Boundary Layer," Atmospheric Science and Power Production, DOE/TIC-27601, U.S. Department of Energy.

Rao, R.S., and H.F. Snodgrass, 1982, PAL-DS Model Including Deposition and Sedimentation, EPA-600/8-82-023, U.S. Environmental Protection Agency, Research Triangle Park, N.C. 
Raynor, G.S., et al., 1979, "Formation and Characteristics of Coastal Intemal Boundary Layers During Onshore Flows," Boundary Layer Meteorology 16:487-514.

Schulman, L.L., et al., 1990, Effects of Structures on Toxic Vapor Dispersion, Final Report, Cont. No. F08635-90-C-397, ESL/AFESC, Tyndall Air Force Base, Fla.

Schulman, L.L., and J.S. Scire, 1980, Buoyant Line and Point Source (BLP) Dispersion Model User's Guide, Document P-7304B, Environmental Research and Technology, Inc., Concord, Mass.

Scire, J.S., and L.L. Schulman, 1981, "Evaluation of the BLP and ISC Models with SF 6 Tracer Data and $\mathrm{SO}_{2}$ Measurements at Aluminum Reduction Plant," APCA Specialty Conference on Dispersion Modeling for Complex Sources, St. Louis, Mo.

Sedefian, L, and E. Bennett, 1980, "A Comparison of Turbulence Classification Schemes," Annospheric Environment 14:741-750.

Smith, R.B., 1990, "Why Can't Stably Stratified Air Rise over High Ground?" pp. 105-107 in: Arnospheric Processes over Complex Terrain, American Meteorological Society.

Spicer, T., and J. Havens, 1989, User's Guide for the DEGADIS 2.1 Dense Gas Dispersion Model, EPA-450/4-89-019, U.S. Environmental Protection Agency, Nov.

Spore, J.W., et al., 1996, In-Facility Transport Code Review, U.S. Department of Energy, July.

Strimaitis, D.G., et al., 1987, EPA Complex Terrain Model Development: Final Report, EPA/600/3-88006, U.S. Environmental Protection Agency, Research Triangle Park, N.C.

The Netherlands Organization of Applied Scientific Research, 1992, Methods for the Calculation of Physical Effects, CPR 14E, 2nd Edition, Directorate-General of Labour of the Ministry of Social Affairs and Employment, The Hague.

Tilton, J.N., and C.W. Farley, 1990, "Predicting Liquid Jet Breakup and Aerosol Formation During the Accidental Release of Pressurized Hydrogen Fluoride," Plant/Operations Progress $9(2): 120-124$.

TNO - See The Netherlands Organization of Applied Scientific Research.

Tumer, D.B., 1964, "A Diffusion Model for an Urban Area," Journal of Applied Meteorology 3:83-91.

Tumer, D.B., 1970, Workbook of Atmospheric Dispersion Estimates, Office of Air Programs Publication No. AP-26, U.S. Environmental Protection Agency, Research Triangle Park, N.C.

Tumer, D.B., 1994, Workbook of Atmospheric Dispersion Estimates - An Introduction to Dispersion Modeling, 2nd Edition, Lewis Publishers, CRC Press. 
U.S. Department of Energy, 1994, Chemical Safety Vulnerability Working Group Report, DOE/EH-0396P, Vol. 1 of 3, Sept.

U.S. Environmental Protection Agency, 1987, On-Site Meteorological Program Guidance for Regulatory Modeling Applications, with Addendum (Feb. 1993) and corrections (Sept. 1993), Research Triangle Park, N.C.

U.S. Environmental Protection Agency, 1987/1993, On-Site Meteorological Program Guidance for Regulatory Modeling Applications, EPA-450/4-87-013, Feb./Sept.

U.S. Environmental Protection Agency, 1992, Workbook of Screening Techniques for Assessing Impacts of Toxic Air Pollutants, EPA-454/R-92-024.

U.S. Environmental Protection Agency, 1993a, An Evaluation of Solar Radiation/Delta-T Methods for Estimating Pasquill-Gifford ( $P-G)$ Stability Categories, OAQPS EPA-454/R-93005 , Oct.

U.S. Environmental Protection Agency, 1993b, Guidance on the Application of Refined Dispersion Models for Hazardous/Toxic Air Releases, EPA-454/R-93-002, May.

U.S. Environmental Protection Agency, 1995, User's Guide for the Industrial Source Complex (ISC3) Dispersion Model, Vols. I\&I, EPA-454/B-95-003a, Sept.

U.S. Environmental Protection Agency, 1996a, RMP Offsite Consequence Analysis Guidance, May 24.

U.S. Environmental Protection Agency, 1996b, "Guideline for Air Quality Modeling, Supplement C," 40 CFR Part 51652, Appendix W, Federal Register, Washington, D.C., Aug. 12.

U.S. Environmental Protection Agency and National Oceanic and Atmospheric Administration, 1995, ALOHA (Areal Locations of Hazardous Atmospheres), User's Manual, June.

van Ulden, A.P., 1974, "On the Spreading of a Heavy Gas Released near the Ground," Proceedings from the 1st Intern Symposium on Loss Prevention and Safety Promotion in the Process Industries, C.H. Buschman (editor), 12:2289-2300.

Venkatram, A., 1986, "An Examination of Method to Estimate the Height of the Coastal Internal Boundary Layer," Boundary-Layer Meteorology 36:149-156.

Weber, A.H., and K.R. McDonald, 1977, "Turbulence Classification Schemes for Stable and Unstable Conditions," Proceedings of the Joint Conference on the Applications of Air Pollution Meteorology, Salt Lake City, Utah, Nov. 29 - Dec. 2.

Whitacre, C.G., et al., 1987, Personal Computer Program for Chemical Hazard Prediction (D2PC), CRDEC-TR-87021, Chemical Research, Development, and Engineering Center, U.S. Army Armament Munitions Chemical Command, Jan. 
Wilcox, 1994, Turbulence Modeling for CFD, DCW Industries, La Cañada, Calif., Nov.

Wilson, D.J., 1979, "Flow Patterns over Flat-Roofed Buildings and Application to Exhaust Stack Design," ASHRAE Transactions 85(2):284-295.

Woodard, J.L., et al., 1995b, "Modeling and Validation of a Dispersing Aerosol Jet," Joumal of Hazardous Materials 44:185-207.

Woodard, J.L., and A. Papadourakis, 1995a, "Reassessment and Reevaluation of Rainout and Drop Size Correlation for an Aerosol Jet," Joumal of Hazardous Materials 44:209-230.

Yamada, T., and S. Bunker, 1988, "Development of a Nested Grid, Second Moment TurbulenceClosure Model and an Application to the 1982 ASCOT Brush Creek Data Simulation," Joumal of Atmospheric Science 27:562-578. 


\section{INDEX}

\begin{abstract}
ADAM model ...xi, xix, xxi, 3-4, 3-5, 3-15, 3-16, 4-7, 5-1, ..5-2, 5-3, 5-8, 5-9, 5-11, 6-13, 6-14, 6-16 Advection $x i, 2-15,2-21,3-10,6-5,6-7,6-23,7-1,7-3$

travel time. $2+19,2-21,2-22,3-11,3-13$ Aerosol $x i, x v, x \times i i, 2-2,2-4,2-5,2-6,2-8,2-9,2-12$, $3-8,3-93-13,3-14,3-16,3-18,4-6,4-8,4-10$. .. 5-5, 5-11, 5, 12, 5-26, 5-27, 5-28, 6-4, 6-8, 6-22. $6-23,6-24,6-30,6-32,6-3-1.7-1,8-1,8-4,8-5,8-7,8-9$ aerosol evaporation ...2-2

- droplet size $2-4,2-5,6-34$

- rainout (see aiso fallout mechanisms and pool evaporation)
\end{abstract} $.2-5,3-9,3-14,6-22,6-34,8-9$

- two-phase jet. $2-5,4-6$ AFTOX model …........xi, xix, xxi, 1-10, 3-5, 3-15, 3-16, 6-16, 6-29 ALOHA model ..... xi, xix, xxi, 1-10, 3-4, 3-5, 3-6, 3-13, 3-18, 5-1, 5-2, 5-3, 5-10, 5-11, 5-12, 6-13, 6-16, 6-19, 6-20. $6-21,6-22,6-24,6-27,8-3,8-8$ AQPAC model. $x i, x i x, x x i, 3-15,3-16,6-29$ ARAC I model (GC-MATHEW/ADPIC) ........ xix, xxi, 3-15, 3-16, $6-29,6-30$ A veraging time 2-19, 2-20, 2-22, 3-7, 3-8, 3-9, 3-13, $4-8,5-3,5-6,5-8,5-13,5-14,5-15,5-16,6-5$, $6-11,6-19,6-22,6-23,6-26,7-1,8-1$ Best Practices xxi, 1-3, 1-6, 1-7, 1-10,3-1, 3-2, 6-1,6-2 Beyond design basis incident (BDBI) xi, 6-2, 6-3, 6-4, 6-8. $6-9,6-11,6-12,7-2$ Boussinesg approximation ..2-18, 7-2 $\mathrm{B} \& \mathrm{M}$ (Britter and McQuaid) model $2-18,3-13$ Building Aerodynurics 6.11 - dowriwash effects ..2-7, 2-9, 2-10, 2-18, 3-6, $3-11,3-13,3-15,5-10,6-29,8-2$ - cavity region 2-10, 5-10,6-11, 6-33

- wake region. $2-9,2-10,3-16,3-19,4-8,5-2$, $.5-5,5-10,6-10,6-11,6-22,6-29,6-33,8-5,8-6$ Buoyancy-dominated plume $2-6,2-7,2-9,7-1$ CALPUFF model. xii, xix, xxi, 3-4, 3-6, 3-7, 5-1, 5-2, 5-3, 5-7, 5-8, 5-9, 5-10, 5-11, 6-13, $6-14,6-19,6-20$ CASRAM model. xii, xix, xxi, 3-4, 3-7, 5-1, 5-2, $5-3,5-7,6-13,6-14,6-20$ Chemical reactivity 7-6 continuous retease $2-2,2-21,3-7,3-12,3-13$ $3-15,3+17,6-5,7-3$ Computational fluid dynarrics (CFD). $6-11,7-2$ Concentration flucruavions 3-4, 3-11, 5-3, 5-6, 8-1 CTDMPLUS mode] xii, xix, xxi, 3-15, 3-17, 6-29 DEGADIS model $x i i, x i x, s x, x x i, 1-10,3-4,3-5,3-8,5-1$, $.5-2,5-3,5-4,5-8,5-9,5-10,5-11,5-12$, 6-10, 6-13, 6-19, 6-21, 6-22, 6-24, 6-27, 6-32, 6-37, 8-7 Dense gas releases (also heavier-lhan-air). 2-7. 2-8, 2-11, 2-18, 3-13 - dispersion models. .. x, xy, xvii, xviii, xxii, xxiii, 1-1, $1-5,1-8,1-9,2-1,2-14,2-19,3-1,3-2,3-17$. 3-19, 4-6, 5-5, 6-1, 6-2, 6-3, 6-4, 6-9, 6-10, $6-11,6-15,6-17,6-21,6-34,6-36,7-10,8-1$. $8-2,8-4,8-5,8-8$ - jets 2-1, 2-2, 2-4, 2-6, 2-8, 2-11, 2-12, 2-15, $.2-17,3-2,3-5,3-9,3-12,4-6,5-4,5-10$, .6-16, 6-22, 6-27, 8-5 - plumes $x x i, 2-1,2-2,2-3,2-6,2-7,2-9,2-11$, 2-13, 2-15, 2-17, 3-5, 3-16, 4-6, 5-7, 5-10, $.6-5,6-7,6-2 t, 6-30,7-1,7-7,8-4,8-5$

Dense gasivapor. $. .7-3$ Deposition (dry) xv, xxii, 2-6, 3-6, 3-11, 3-14, 3-18, $3-19,3-20,5-2,5-5,5-6,6-10,6-26$ $6-29.6-33,7-9,8-6$ Deterministic model $7-3,7-10$ Dispersion coefficient $(\sigma)$ $2-17,3-6,7-3$ Downwash Effects ..5- 10 DRIFT model xii, 6-13 EMGRESP model

Enurairment xiii, xix, 3-15, 3-17, 3-18, 6-30 $2-5,2-8,2-10,2-11,2-12,2-15,2-17,2-19$, $3-8,3-12,5-5,7-3,7-9,8-6$ ERPG xiii, 2-21, 6-4, 6-5, 6-12, 6-20, 8-3

Eulerian coordinate system $. .7-3$ Exposure time $2-1,2-19,2-20,2-21,6-4,7-8$ f-N Curves xiii, 7-4

Follout mechanism. $.2-6$

FEM3C nodel. xii, xix, xxi, 2-10, 3-4, 3-8, 3-9,

.5-I, 5-2, 5-3, 5-9, 5-10, 5-11, $6+13,6-14,6-22,6-23,6-29,7-2$ Flashing $2-2,2-4,2-5,4-6,7-4,8-1,8-5$

- tlashed fraction ,$\ldots 2-4$ 
Jerosol fomation

xxii, 2-2, 2-4, 4-6, 6-32, 6-33, 6-34, .., 6-35, 8-4, 8-7

Fricrion velocicy 2-6, 6-8. 6-9, 7-3, 7-4, 7-5, 7-7

Fumigaion effects $3-20,7-4,7-6,8-5$

GAUS1 model xiii, xix, 3-15, 3-18, 6-29

Gaussian model $3+18,6-19,7-5,7-10$

Gaussian plume xiv, 2-16, 2-19, 3-17, 5-9, 6-11, $.7-5,7-9,7-10,8-4$

Gaussian plume mode! 5-9. 8-4

- dispersion coefficieuts $2-17,2-18,3-6$

Gaussian puff. $3-14,3-16,3-18,6-26,7-5$ GC-MATHEW/ADPIC xix, xxi, 3-15, 3-16, 6-29, 6-30 Gradient trasport. $4-4,7-5,7-6$ HARM-II model xix, 1-10, 3-15, 3-18, 6-29

Hazard zone..... 1-2, 2-11, 3-7, 3-16, 5-2, 5-6, 6-4, 6-11, 6-12, 7-5 heavier-than-air . $2-2,3-13,5-4,6-10$ HGSYSTEM model. xi, xii, xir, xY, xix, xxi, 1-10. 3-4, 3-9, 3-10, 5-1, 5-3, 5-4, 5-8, 5-9, 5-10, $5-11,5-12,6-4,6-9,6-11,6-13,6-19,6-21,6-21$, $6-23,6-24,6-27,6-33$

Honogeneity ..7-5 HOTMLACRAPTAD nodel ........xix, xxi, 3-4, 3-10, 5-1, 5-7, 6-13, $6-14,6-24,6-25$ Induscrial source complex (ISC) model ..................... xiv, 2-6, 2-10. $4-8,6-11,6-33,6-34,8-7,8-8$ INPUFF model $x i v, x i x, x x i, 1-10,3-4,3-10,3-1 t$. 5-1,5-2, 5-3, 5-7, 5-8, 5-9, 5-10, 5-11. $6-13,6-1+, 6-20,6-25,6-26$ Input data...................... $x x, 3-7,3-10,4-7,6-2,6-25,6-27,6-28$ - meteorological daha $2-10,3-16,3-17,3-19$. $4-4,6-7,6-28,6-33,7-7,7-8,7-10$ Instantaneous release $2-21,3-5,3-12,3-13,3-18$. $6-25,6-34,8-2$ Inversion layer $7-6.7-7$ Jers $2-1,2-2,2-4,2-6,2-8,2-11$, 2-12, 2-15,2-17, 3-2, 3-5, 3-9, 3-12 4 $4-6,5-4,5-10,6-16,6-22,6-27,8-5$

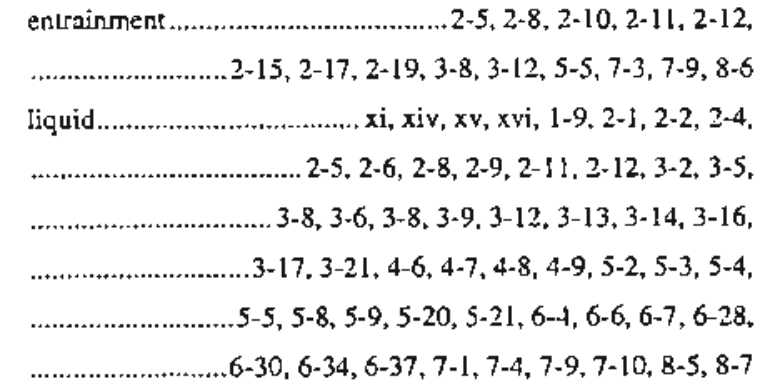

- $\quad$ wo-phase ................ xii, 2-1, 2-4, 2-5, 2-7, 2-12, 3-1, 3-2, 3-5. $3-14,4-6,5-4,5-11,6-4,6-19,7-10,8-5$

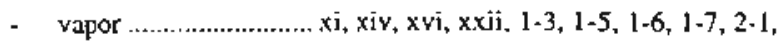
.. 2-2, 2-4 through 2-12, 2-14 through 2-19. 3-5, 3-8, 3-9, 3-11, 3-12, 3-14, 3-18, 3-20, 3-21, 4-6, 4-8, 5-3, 5-4, 5-5, 5-6, 5-12, 6-1, $6-4,6-6,6-7,6-8,6-9,6-10,6-11,6-12,6-14$, $6-22,6-23,6-25,6-28,6-29,6-30,6-33,6-34$, $6-37,7-1,7-2,7-3,7-5,7-6,7-7,7-8,7-10,8-1$, 8-3, 8-4, 8-7

K-Theory 3-8, 6-33, 7-6

K-E Theory $2+13,3-8,6-23,6-33,7-6$ Lagrangian coordinare system ...7-6 Lift-off (exolhermic/chemical). $2-9,3-9,5-2,5-5,7-6$ MARSS model. $x i, x i y, x y, x i x, 3-15,3-19,6-30$ Meterological condicions. xii, 2-11, 3-6, 3-10, 3-13. 3-14, 3-20, 3-21, 5-5, 5-6, 6-4, 6-6, 6-7, 6-10,6-22, 6-28, 7-3, 7-7, 7-8

Meteorological data .2-10, 3-16, 3-17, 3-19, 4-4, 6-7. $6-28,6-33,7-7,7-8,7-10$

Merosology $x y, x v i i, 2-1,2-10,2-11,2-13,2-16$, 3-7, 3-11, 3-14, 3-19, 5-3, 5-6 6-6, $6-12,7-1,8-2,8-4,8-5,8-6,8-7,8-8$

- ambient temperature ......2-12, 4-7, 4-8, 5-8, 6-6, 6-7, 6-8, 6-21

. armospheric pressure ......................................... 2-4, 2-12, 3-2

- atmospheric rurbulence and diffusion ................................2-12 relative humidiry $2-5,2-6,2-10,2-12,2-14,6-4$, $6-5,6-7,6-8$ ground-surlace effects $2-10,2-13$ Mixing depth (z). $.7-7$ Momentura-dominated plume $2-4,2-6,2-7,7-7$ Monin-Obukhov length (L). $2-14,7-7$ Negarively buoyant - see Dense gas releases and Dense gas/yapor Neutrally buoyant gas/vapor .... x viii, 2-1. 2-7, 2-8, 2-9, 2-11, 2-19, $3-6,3-8,3-9,3-11,3-12,3-13,3-17$. $3-19,3-20,5-4,5-9,6-5,6-16,6-25$, 6-26. 6-27, 7-6. 7.7

Plume meander $3-13,5-3,5-6,7-7,7-10$

Plumes $x x i, 2-1,2-2,2-3,2-6,2-7,2-9$ $2-11,2-13,2-15,2-17,3-5,3-16$ 4-6, 5-7, 5-10, 6-5, 6-7, 6-21, 6-30, 7-1, 7-7, 8-4, 8-5

Pool evaporation $2-2,3-1,3-5,3-7,5-13,5-14$ 5-15, 5-16, 6-4, 6-6, 6-7, 6-8 ground surface eftects 2-10, 2-13 insolation $2-13,2-15,6-5$ 
Positively buoyant gas/vapor.... 2-1, 2-7, 2-9, 2-15. 2-18, 6-29.7-7 Probit.

Prognostic model .7-8

Public receptor $7-8$

PUFF-PLUME model $x v, x i x, 3-15,3-19,3-20,6-30$

Release duracion 2-1. 2-19, 3-6, 3-7, 3-8, 3-13, 6-7 Risk analysis $2-20,7-9,8-1$

Roughness parameter or length $\left(\mathrm{z}_{0}\right)$. $6-5,6-8,6-9,6-10$, 6-33, 7-9

RTVSM model . $x v, x i x, x i, 3-15,3-20,6-29$ Second-order closure $x v, 3-11,6-26,7-9$ Spulls xvii, xviii, xxiii, 1-3, 1-9, 1-10,

2-2, 2-4, 2-11, 3-2, 3-3, 3-4, 3-5, 3-7, 3-14, $3-16,3-17,3-18,3-21,4-2,4-4,4-6,5-2,5-3$. $5-4,6-2,6-3,6-4,6-5,6-20,6-21,6-28,6-29$, 6-37, 7-10, 8-2

- liquid, subcooled. $2-2,2-4,2-7,2-12,3-2$

- liquid, supertheated $2-1,2-4,2-5,2-7,2-12,3-2$

Fallout roechanisms. . 2-6

- partial reflection. ...2-6

- source depierion 2-6

Sampling time: (see averaging time) 2-20 Spills front-end $3-7,3-16,5-2,5-3,5-4,6-21,6-29,6-37$
Source-term. 2-1, 2-2, 3-3, 3-9, 3-17, 3-18, 4-2, 4-4, 6-11, $6-20,6-22,6-35,6-37,7-5,8-3,8-5$ Sability classificarion methods $2-13,8-6$

- Pusquill-Gifford..........xiv, 2-13, 3-11, 6-6, 6-11, 6-21, 7-4, 8-8

- turbulence. xiii, 2-6, 2-8, 2-9, 2-10, 2-1 I, 2-12, $2-13,2-14,2-15,2-17,2-19,2-20$, $3-7,3-8,3-10,6-5,6-8,6-9,6-10,6-11$, $6-12,6-20,6-23,6-24,6-25,6-33,6-34$, $7-2,7-3,7-4,7-5,7-6,7-9,7-10$, $8-5,8-6,8-7,8-8,8-9$

Stochastic model $7-10,8-5$

Straight-line Gaussian plumejpuff $.7-10$

TSCREEN model $x v, x i x, x x i, 3-4,3-13,5-1,5-2$, $.5-3,5-7,5-9,5-10,5-11,6-13,6-14$, 6-27, 6-28

Toxic load $x x$ ií, 2-20, 2-22, 5-3, 5-6, 6-4, 7-8

Two-phase releases $2-4,2-5,2-7,7-10$ Uncertainty $x \mathrm{ii}, 2-15,3-12,4-5,5-3,5-6$, $6-2,6-22,6-26,6-32,7-10$ VDI modet xvi, xix, $x x i, 3-15,3-20,3-21,6-29$ VLSTRACK model xix, 3-4, 3-14, 3-15, 5-1, 5-7, 5-9, 5-11, 6-13, 6-15, 6-28

"Worst-Case" $x \times i, 5-3$ 
